\title{
Functional study of potential sHSPs in Arabidopsis and tomato under environmental stress
}

\author{
Dissertation \\ for the award of the degree \\ "Doctor rerum naturalium" \\ of the Georg-August-Universität Göttingen
}

\begin{abstract}
Within the Binational Doctoral Program
on Molecular Biosciences and Biomedicine

of the Georg-August-Universität School of Science (GAUSS) and

Universidad Nacional de Rosario (UNR)
\end{abstract}

Submitted by

Mariela Raquel Escobar

from Barreal (San Juan), Argentina

February 2019 


\section{Thesis Committee}

Dr. Estela Marta Valle

(Instituto de Biología Molecular y Celular de Rosario, UNR-CONICET).

Prof. Dr. Ivo Feussner

(Department of Plant Biochemistry, Albrecht-von-Haller-Institute for Plant Science, University of Göttingen).

Dr. Juan Pablo Amelio Ortiz

(Instituto de Investigaciones en Ciencias Agrarias de Rosario, UNR-CONICET).

\section{Members of the Examination Board}

Dr. Juan Pablo Amelio Ortiz

(Instituto de Investigaciones en Ciencias Agrarias de Rosario, UNR-CONICET).

Dr. Norberto Daniel lusem

(Instituto de Fisiología, Biología Molecular y Neurociencias, UBA-CONICET).

Dr. Nestor Carrillo

(Instituto de Biología Molecular y Celular de Rosario, UNR-CONICET).

Dr. Till Ischebeck

(Department of Plant Biochemistry, Albrecht-von-Haller-Institute for Plant Science, University of Göttingen).

Prof. Dr. Andrea Polle

(Department for Forest Botany and Tree Physiology, Büsgen Institute, University of Göttingen). 


\section{Table of contents}

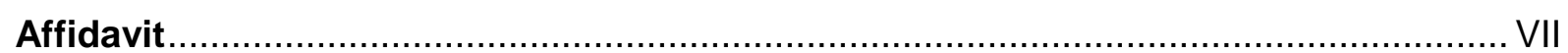

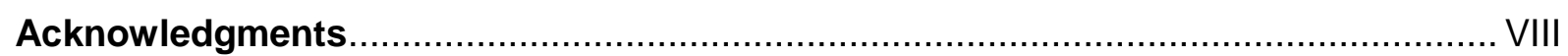

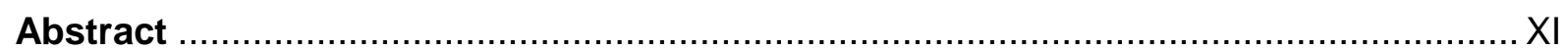

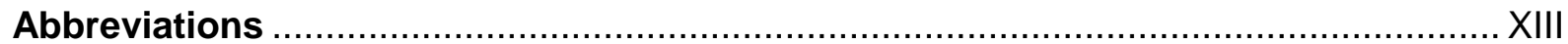

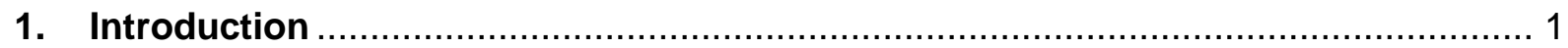

1.1. The small heat shock protein family. ............................................................ 1

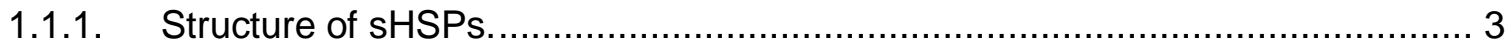

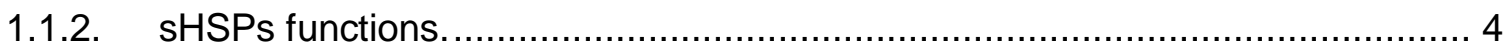

1.1.3. Regulation of SHSPs and their role in stress response................................ 5

1.2. Genomic organization of sHSPs and bidirectional promoters (BDPs) $\ldots \ldots \ldots \ldots \ldots \ldots \ldots$

1.3. Mitochondrial small heat shock proteins. ....................................................... 9

1.4. Cold stress and chilling injury in plants.............................................................

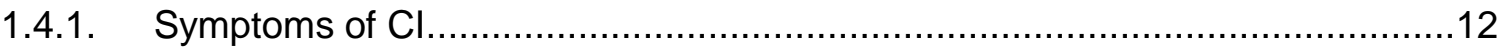

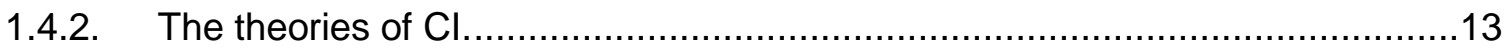

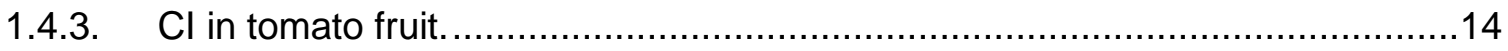

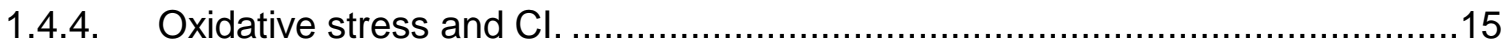

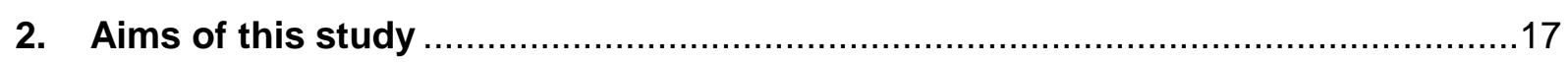

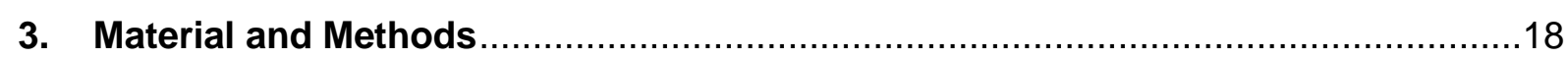

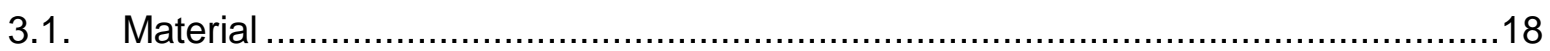

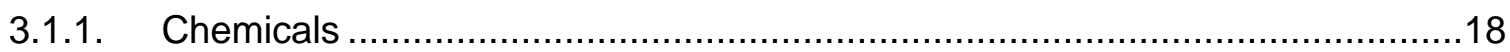

3.1.2. Kits, enzymes and commercial reagents ................................................18

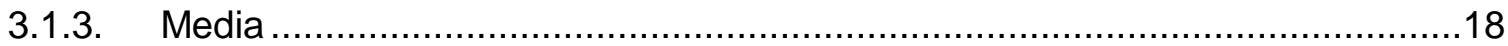

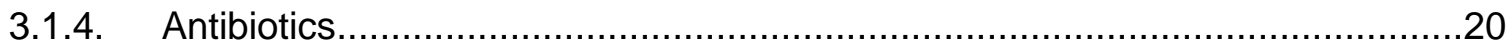

3.1.5. Sodium dodecyl sulfate-polyacrylamide gel electrophoresis (SDS-PAGE) ......20

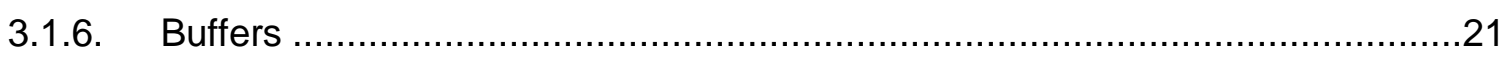

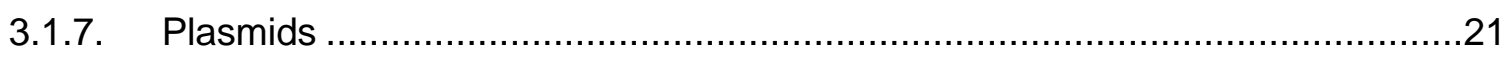

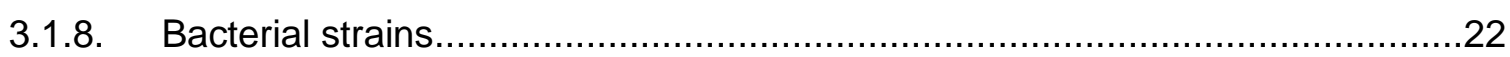

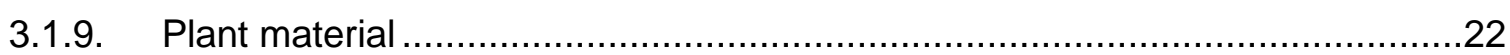

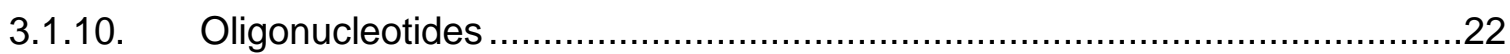

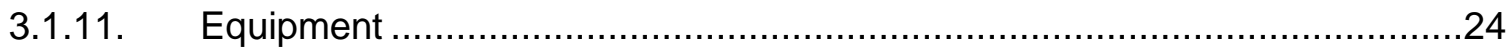

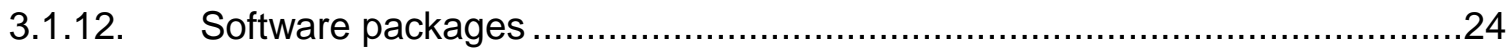

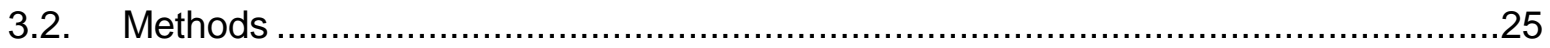

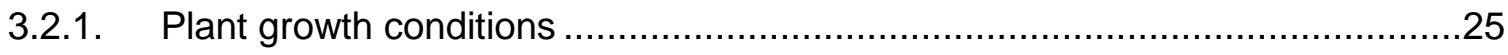

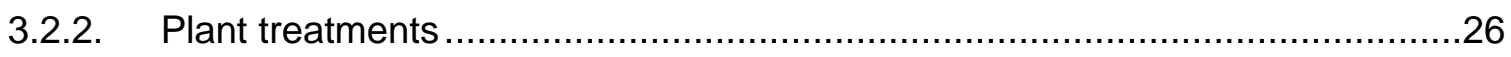




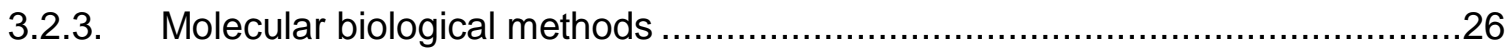

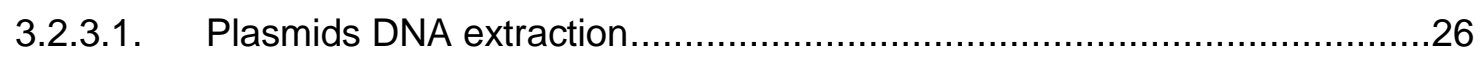

3.2.3.2. Isolation of DNA from $A$. thaliana and tomato plants ............................27

3.2.3.3. Isolation of RNA from tissues plants ..............................................

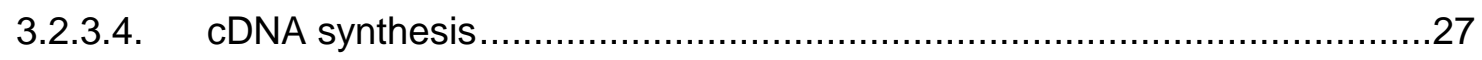

3.2.3.5. Quantitative real-time PCR (q-PCR) .............................................. 28

3.2.3.6. Polymerase chain reaction (PCR) ......................................................28

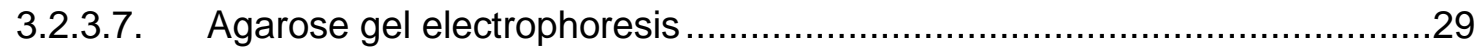

3.2.3.8. Restriction, ligation and subcloning of DNA

3.2.3.9. Generation of microRNA silenced plants ...............................................

3.2.3.10. Construction of promoter-GUS fusion lines. ......................................

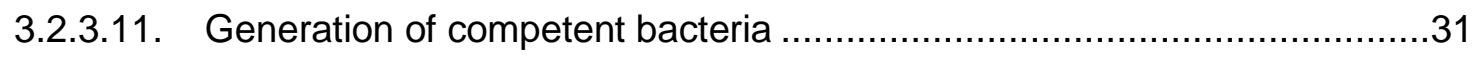

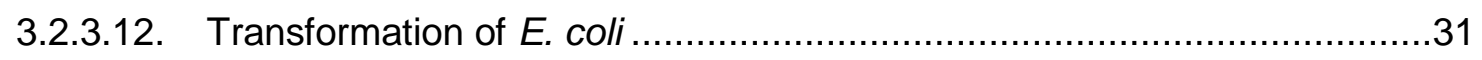

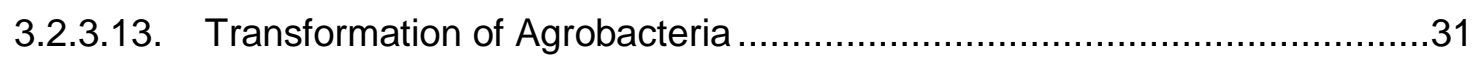

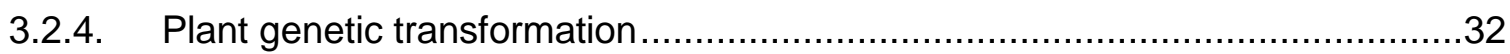

3.2.4.1. Agrobacterium-mediated transformation of $A$. thaliana ............................32

3.2.4.2. Agrobacterium-mediated transformation of tomato .................................

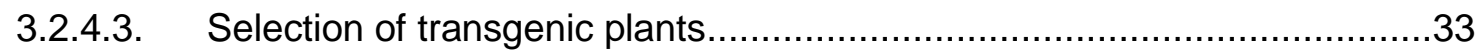

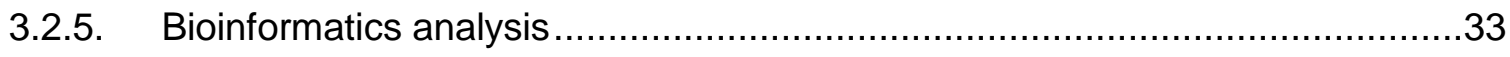

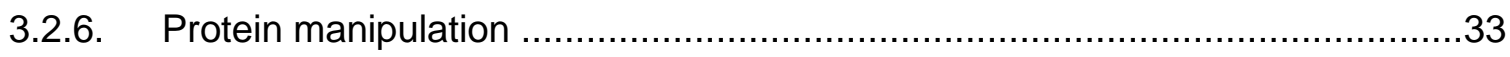

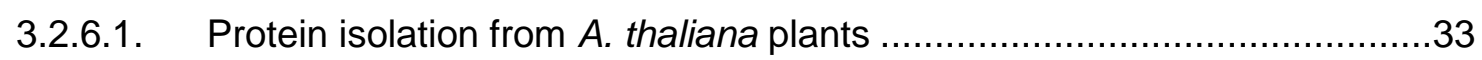

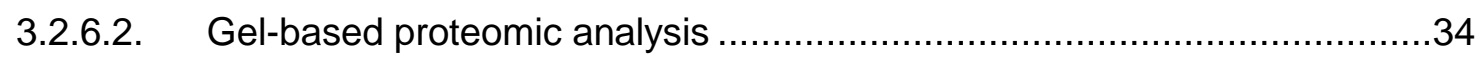

3.2.7. Metabolite profiling by gas chromatography coupled to mass spectrometry (GC-

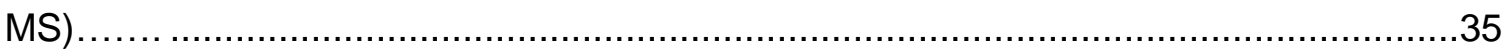

3.2.8. Analysis of the tomato lipidome by UPLC-nanoESI-MS/MS ..........................36

3.2.9. Color and pigment content determinations. ...............................................

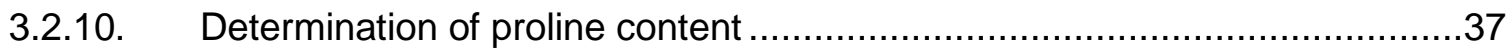

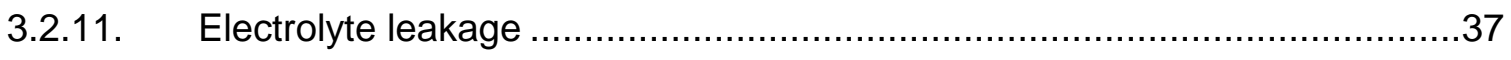

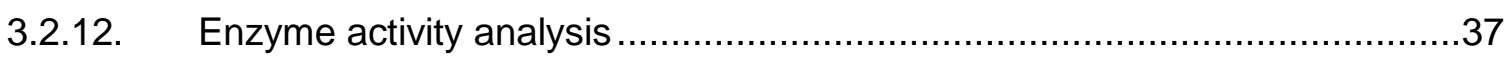

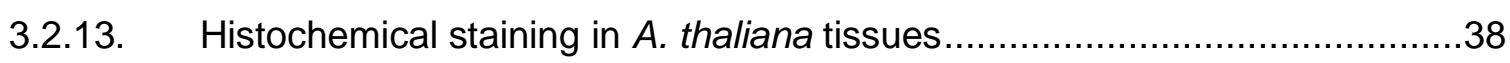

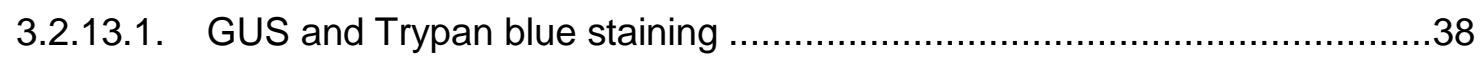

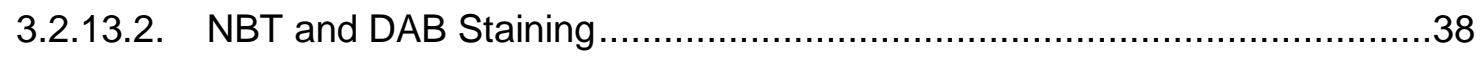

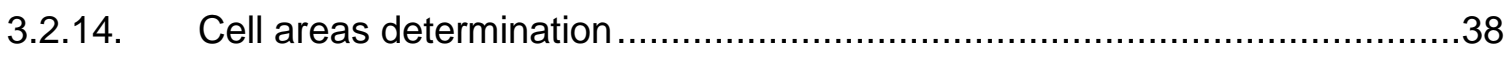

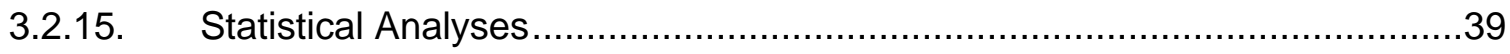

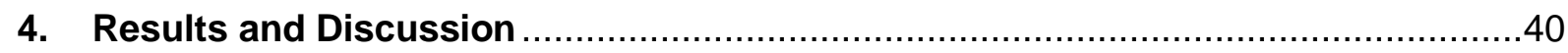

4.1. Putative bidirectional promoters regulate the expression of ACD genes in Arabidopsis thaliana under abiotic stress. 


\subsubsection{Characterization of four genes encoding ACD proteins with head-to-head}

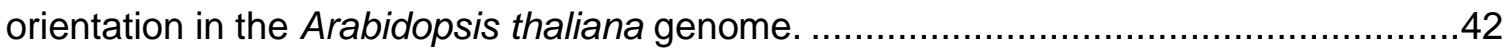

4.1.2. Expression analysis of bi-directional genes under abiotic stress conditions.....43

4.1.3. Analysis of four putative bidirectional promoters in $A$. thaliana. ......................48

4.1.4. The activity of the putative bidirectional promoters in $A$. thaliana seedlings.....58

4.1.5. Discussion

4.2 Functional characterization of mitochondrial small heat shock proteins in

Arabidopsis thaliana.

4.2.1. sHSP23.5, sHSP23.6 and sHSP26.5 are putative mitochondrial sHSPs in $A$.

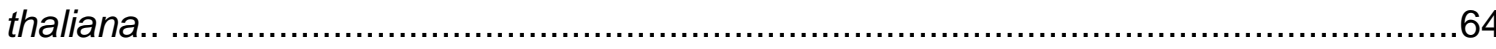

4.2.2. $\quad$ sHSP23.5, sHSP23.6 and sHSP26.5 are regulated by abiotic stresses. .........66

4.2.3. Artificial microRNAs were designed for sHSPs-M genes. ..............................69

4.2.3.1. Knockdown mutants display altered growth phenotype. ..........................71

4.2.3.2. Roots as the main altered tissue in amiR26.5 plants. ...............................75

4.2.3.3. Analysis of knockdown mutants at the protein level..............................76

4.2.3.3.1. Overview of the proteomes of Arabidopsis amiRsHSP-M plants. .........77

4.2.3.3.2. Deficiency of individual sHSPs generates distinct proteome response. 78 4.2.3.3.3. Proteome of the double amiR23.5/23.6 mutant widely differs from the proteome of single amiR23.5 and amiR23.6 mutants.

4.2.3.3.4. Common changes in the proteome of amiR23.5/23.6/26.5 and single amiR mutants.

4.2.3.4. Reduction in the SHSP-M content leads to profound metabolic alterations...

4.2.3.5. amiR-triple mutant exhibited up-regulation of several photosynthetic related proteins.

4.2.3.6. Up-regulation of ROS detoxifying enzymes in the amiR mutants

4.2.3.7. Cell membranes are highly affected in the knockdown sHSP-M mutants. 96

4.2.4. Discussion .99

4.3. Mitochondrial small heat shock protein and chilling tolerance in tomato fruit ........105

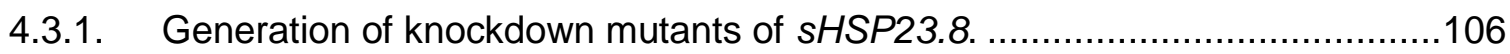

4.3.2. amiR23.8 fruit is more susceptible to chilling injury than WT fruit. .................107

4.3.3. Antioxidant system of amiR23.8 fruit is slightly altered after chilling storage.. 109

4.3.4. Lipid composition of amiR23.8 fruit after chilling stress differs from WT fruit. 110 4.3.4.1. amiR23.8 fruit had altered amounts of glycerolipids and showed differential response to chilling stress.

4.3.4.2. Lipidome response of WT and amiR23.8 fruit differed depending on the lipid class... 


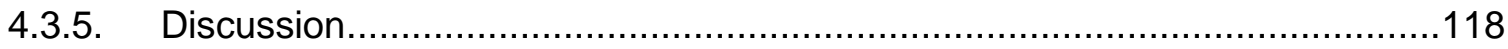

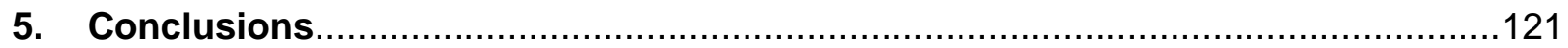

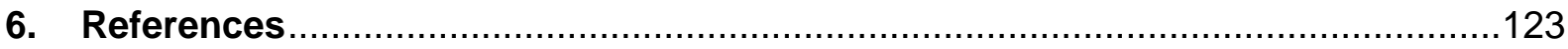

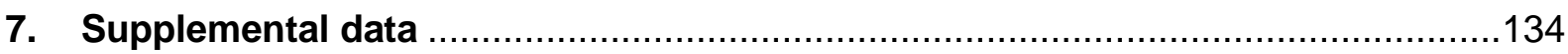




\section{Affidavit}

Hereby, I declare that this dissertation entitled "Functional study of potential sHSPs in Arabidopsis and tomato under environmental stress" has been written independently and no other sources and aids than quoted.

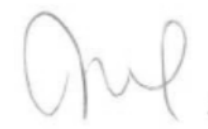

Mariela Raquel Escobar

Rosario, $5^{\text {th }}$ February 2019 


\section{Acknowledgments}

I would like to thank in the first place my supervisors Dr. Estela Valle in Argentina and Dr. Ivo Feussner in Germany, for giving me this project and letting it evolve with patience and a great deal of support.

I would also like to thank CONICET and DAAD for the financial support during my Ph.D., as well as both the National University of Rosario and the Georg-August-Universität, and the staff from GAUSS and the Binational Ph.D. program for all the assistance during these years.

I am also indebted to Dr. Juan Pablo Ortiz as a member of the thesis committee for his interest in the project and very helpful and interesting discussions.

I would like to thank very especially Dr. Maria Inés Zanor and Dr. Silvana Boggio for all the enriching discussions and suggestions to my work, and for being there willing to listen and help me whenever I needed it.

Thanks a lot, to Dr. Gisela Ferraro and Dr. Virginia Osella for receiving me in the lab, teaching me and providing me with many technical advices and tips, valuable resources that cannot be found in any book.

I would like to thank Dr. Cornelia Herrfurth and Dr. Pablo Tarazona for the lipidomic measurements and data analysis, their guidance in this part of my work, lots of helpful advice in the lab as well as their patient and very kind way of dealing with so many students at the same time.

Thanks to Dr. Oliver Valerius and Dr. Kersten Schmitt whose practical and theoretical advice helped me in planning the proteomic experiments, and who performed all the LC-MS/MS analyses of the protein samples.

I am very grateful to Dr. Kirstin Feussner not only because of her help in the metabolomic measurements not included in this work, but most importantly for being so nice and patient from the first moment I arrived in Göttingen and assisted me with all my needs.

I want to especially thank Sabine Freitag for her practical support with lipid extractions and constant willingness to help, and Dr. Ellen Hornung for helping me a lot with her scientific 
experience and advice, and considering me as part of the group in all the social meetings and plans. I found incredible how she deals with so many questions and students at the same time while working hard, and still staying in such a good mood.

I also appreciate all the practical help that I got from Diego Aguirre and Susanne Mester in terms of plant care and seed collecting - thank you!

In Göttingen,

I want to express my gratitude to all the members of the Prof. Feussner's lab, especially the people on the second floor, Sven, Hanno, Amélie, Dimitrij and Jennifer who have really made it a great place to work.

My special thanks to Noku, Yi-Tse, Milena and Alaa, very good and close friends I made in Göttingen. Thanks for all the chats, jokes, cocktails, beers and butter-pretzels we shared.

Outside of the lab, I would like to say thanks to Giovanna, Charlotte, Miroslava, Senghour and Jeanine for the trips, walks, dinners, beers and all the time we spent together! You were my family there!

En Argentina,

Quiero agradecer inmensamente a todos mis compañeros y amigos del lab 2. Sin dudas una de las cosas más lindas que me llevo de estos cinco años. Gracias a Gi, Vir, Telmis, Agus, Vicky, Diego, Anabel, Ivi, Arleth, Ceci, Pau, Diane, Alejo y a los recientemente incorporados Lara Y Diego Dj. Gracias por todos los lindos y divertidos momentos, y por estar también en aquellos momentos que se volvieron un poco difíciles. Diego Alberto, muchas gracias por la paciencia, las ideas y toda la ayuda en los experimentos con Arabidopsis.

¡Gracias a mi hermana del doct, Lau! Gracias por tu gran ayuda con la transformación de tomate, por cuidar de mis plantas en mi ausencia y por cada vez que me ayudaste con cosas del lab. Gracias por estar siempre y ayudarme en todo, y por malcriarme con las chocotortas más deliciosas que he probado.

No puedo dejar de agradecer a Ingrid por acompañarme en este camino que transitamos juntas entre Göttingen y Rosario. Y a Renzo, siempre presente e incondicional, incluso estando a miles de kilómetros. 
Santi, gracias por tu hermosa compañía, por haberme apoyado y escuchado en todo momento y por todos los sacrificios que juntos hicimos para siempre estar juntos en estos años.

Por último, es difícil poner en palabras mi gratitud hacia las personas más importantes que me regaló la vida, mis padres Carlos y Patricia y mis hermanos Ame y Gaby. Gracias por entender y ayudarme siempre, por cada palabra de aliento y las recibidas siempre cálidas. ¡Cada paso siempre con ustedes a mi lado! 


\section{Abstract}

Small heat shock proteins (sHSPs) respond to many environmental stresses, stabilizing early unfolding protein intermediates and avoiding their irreversible aggregation. In plants, organellar sHSPs are a unique cellular feature. The primary structure of sHSPs includes a N-terminal sequence of variable sequence and length, a conserved domain known as $\alpha$-crystallin domain (ACD) and a non-conserved C-terminal sequence. The ACD represents the conserved characteristic present in all SHSPS, although there are other proteins that contain an ACD but are not sHSPs. sHSPs belong to a big superfamily, and the functional and physiological relevance of the different SHSPs remains largely unknown.

The objective of this study was to understand the role of mitochondrial sHSPs in Arabidopsis thaliana and Solanum lycopersicum under environmental stresses and to characterize putative bidirectional promoters driving the expression of ACD proteins with head-to-head orientation. This work covers aspects from the genomic organization and function of SHSPs-M in Arabidopsis to the role of SHSPs-M in chilling stress of tomato fruit. To perform all the experiments, Arabidopsis and tomato mutants using artificial microRNA technology were generated and analyzed in their proteome, metabolome, and lipidome.

In the first part of this work, the functional characterization of head-to-head oriented genes encoding ACD proteins and the correspondent intergenic regions was performed. Four different bidirectional promoters in the A. thaliana genome, including the one of At5g51440 that encodes a mitochondrial sHSP (sHSP23.5), were successfully identified and characterized. The data suggest that the bidirectional promoter contained in the pair At5g51430-At5g51440 is strongly heat induced in one direction but not in the other. The promoter of At1g06460-At1g06470 showed comparable high activity in both directions and thus has a great potential to be used in genetic engineering. The other two promoters showed greater strength in one side and can be considered as asymmetric bidirectional promoters. This functional study of the promoters revealed the biotechnological potential of them because they can be induced specifically in a certain condition (such as high temperature) in one or two directions when it is required.

In the second part, functional characterization of the mitochondrial sHSPs under stress conditions and during $A$. thaliana development is presented. Three gene paralogues were found in Arabidopsis (At5g51440, At4g25200, and At1g52560), and artificial microRNA were used to generate knock-down mutants (single, double and triple amiR). The single and double amiRs (for sHSP23.5 and SHSP23.6) did not show evidently affected phenotype, probably because of functional compensation or redundancy of the mitochondrial sHSPs. On the other hand, the triple amiR23.5/23.6/26.5 mutants showed an altered phenotype in the vegetative 
and reproductive stages. They have reduced leaves areas, but not number of epidermal cells per leaf, chlorotic leaves, shorter root, and reduced seed yield when compared to Col-0 plants. Plants of triple amiR were considerably small due to the alteration in the process of cell expansion but not in the cellular proliferation, which indicates a profound alteration in the plant developmental program. Proteomic analysis of the amiR mutants revealed significant upregulation of various metabolism-related proteins and alterations in the abundance of several proteins that are involved in translation and in the ribosome functioning and structure. Triple amiR mutant exhibited a higher number of proteins with differential abundance related to these processes compared to the other single and double amiR23.5/23.6 mutants. Such a wide change in ribosome-related proteins suggests a possible alteration in the proper ribosome function. The data exposed in this work provide evidence of the important roles that SHSPs-M may play, not only in the heat response but also in the plant development of Arabidopsis. Results demonstrate that a functional compensation might be responsible for the phenotype in mutants lacking single SHSPs-M. However, the reduction of the three SHSPS-M caused a profound disruption in the mitochondria and ribosome functionality that severely affected the energy metabolism and the overall cell homeostasis, leading to alterations in the correct plant development.

In the last part of this work, the functional consequences of the down-regulation of SHSP23.8 in tomato fruit were investigated and analyzed in their phenotype and in their susceptibility to chilling injury. Pre-chilled fruit of amiR23.8 mutant showed higher loss of water and increased ion leakage of pericarp tissue compared to WT fruit. The amiR23.8 fruit deterioration indicates that it is highly susceptible to cold stress and developed chilling injury symptoms. The lipidome of fruit after chilling of amiR23.8 showed altered amounts of glycerolipids, and the level of saturated lipids in amiR23.8 decreased, but not lower than the level in WT under normal conditions. The opposite was found in the relative percentage of unsaturated lipids, having amiR23.8 fruit significantly lower levels in normal conditions and after chilling. The results presented here indicate a differential degradation of extraplastidic and plastidic lipids in amiR23.8 fruit, and alterations in the remodeling of the lipidome after cold stress, which may lead to higher sensitivity to chilling injury. The results discussed here indicate that SHSP23.8 may play an important role in the protection mechanisms against chilling stress in tomato fruit. 


\section{Abbreviations}

$\%:$ Percentage

${ }^{\circ} \mathbf{C}$ : Degrees Celsius

$\mu$ : Micro

ACD: Alpha-crystallin domain

amiR: Artificial micro RNA

ASG: Acylsterolglycoside

BDP: Putative bidirectional promoter

bp: Base pair

Cer: Ceramide

CH: Chilling

Chl: Chlorophyll

Col-0: Columbia 0

CTAB: Cetyltrimethylammonium bromide

d: Day

Da: Daltons

DAG: Diacylglyceride

DGDG: Digalactosyldiacylglycerol

DHSs: DNase I hypersensitive sites

DNA: Desoxy-ribonucleic acid

dNTP: Desoxyribonucleoside triphosphate

DTT: Dithiothreitol

EDTA: Ethylenediaminetetraacetic acid

ESI: Electrospray ionization

et. al.: Et alia

FA: Fatty acid

Fig.: Figure

FW: Fresh weight

g: Gram

GC: Gas chromatography

GIPC: Glycosylinositolphospho-ceramide

GIcCer: Glycosylceramide

GO: Gene ontology

GUS: $\beta$-glucuronidase

h: Hour
HSP: heat shock protein

K: kilo

L: Liter

LB: Luria-Bertani

LC: Liquid chromatography

LCB: Long-chain base

m: Meters

M: Mol per liter

m: Milli

MGDG: Monogalactosyldiacylglycerol

min: Minutes

MS: Mass spectrometry

MS (1/2): Murashige Skoog media

MW: Molecular weight

n: Nano

PA: Phosphatidic acid

PC: Phosphatidyl-choline

PCR: Polymerase chain reaction

PE: Phosphatidyl-ethanolamine

PG: Phosphatidyl-glycerol

pH: Power of hydrogen

PI: Phosphatidyl-inositol

PS: Phosphatidyl-serine

q-PCR: Quantitative real-time polymerase chain reaction

ROS: reactive oxygen species

s: Seconds

SD: Standard deviation

SDS-PAGE: Sodiumdodecylsulfate-

polyacrylamide gel electrophoresis

SE: Sterol esters

SG: Sterolglycoside

sHSP: Small HSP

SQDG: Sulfoquinovosyldiacylglycerol 
TAE: Tris/acetic acid/EDTA

TAG: Triacylglyceride

TEMED: Tetramethylethylenediamine

TF: Transcriptional factor

TFBs: Transcriptional factor binding sites

TSS: transcription start sites
UPLC: Ultraperformance liquid chromatography $\mathbf{v} / \mathbf{v}$ : Volume per volume w/v: Weight per volume WT: Wild type 


\section{Introduction}

Plants, unlike animals, are immobile organisms. As a result, they suffer a wide variety of environmental stresses from germination to senescence. High-light, salinity, drought, high and low temperatures are a few situations that plants have to cope with for surviving. Under such adverse conditions, the photosynthetic antennas absorb photons at a rate that is higher than the capacity of the photosynthetic centres to channel electrons through the electron transport chain mechanisms, resulting in the generation of reactive oxygen species (ROS). Complex defense mechanisms have evolved in plants in response to multiple stressful situations. As a eukaryote, plants have a nuclear genome and two organellar genomes in mitochondria and chloroplasts. Most mitochondrial and chloroplast proteins are encoded in the nuclear genome, and when fully synthesized in the cytosol, proteins need to be imported into the organelles [Jarvis, 2008]. The analyses of transcription profiles in Arabidopsis thaliana, a model plant, revealed the presence of different ways of retrograde control over the nuclear gene expression for mitochondrial or chloroplast proteins [Leister, 2011]. Under different stress situations, the transcriptome of plants showed some common players from transcription factors to heat stress proteins (HSPs), upregulated in a wide variety of stressful situations [Nishizawa, 2006]. In particular, HSPs were highly upregulated under high light and oxidative stress generated in the chloroplast of Arabidopsis [Scarpeci, 2008a, 2008b]. Among them, small HSPs (sHSPs) were upregulated, and two of them were assigned to be localized in the mitochondria. Previously, Neta-Sharir group [Neta-Sharir, 2005] showed that the tomato (Solanum lycopersicum) chloroplast SHSP, HSP21, was induced in the leaves when heat treated, but also in developing fruit during the transition of chloroplasts to chromoplasts when growing under normal conditions. This evidence led to postulate the goal of the present work: to study the role of mitochondrial SHSP in Arabidopsis and tomato under stressful conditions.

\subsection{The small heat shock protein family.}

Functional proteins are the product of the highly efficient biosynthetic system, subjected to strict quality control, composed by chaperones, folding catalysts, and proteases. Protein quality controls are also crucial to eliminate detrimental misfolded/aggregated proteins. Cellular chaperones usually assist other proteins to acquire the final active and functional structures, although they are not part of it [Hartl, 2011]. The HSP families are classified after their molecular size: HSP100s, HSP90s, HSP70s, HSP60s, and the HSP20s or the small HSPs [Wang, 2004b]. The name HSP originates because they are induced on exposure to 
high temperature and play critical roles in the so-called "heat shock response". Upon high stressing temperatures, all organisms alter their gene expression patterns through the activation of the heat shock transcription factors (HSFs) and the binding of these HSFs to heat shock elements. The HSFs can thus up-regulate the heat shock genes leading to the production of heat shock proteins or HSPs [Scharf, 2012]. Although HSPs were first recognized for their up-regulation during heat shock, many reports showed that they are also found in unstressed cells as well as in abiotic and biotic stressed cells [Swindell, 2007].

Most HSP families (the HSP60s, HSP70s, HSP90s, and HSP100s) are highly conserved in many distant organisms; and some of them are known as the most highly conserved protein families [Boorstein, 1994; Stechmann, 2003]. In contrast, the amino acid sequences of sHSPs are highly variable, being the sHSPs family the least conserved of the molecular chaperones [Waters, 1995; Kriehuber, 2010]. However, there are some conserved sHSP features. Firstly, they function as large oligomers although they are named after the size of the monomers (ranging from 15 to $42 \mathrm{kDa}$ ) [van Montfort, 2002]. Secondly, all sHSPs share a conserved $\alpha$ crystallin domain (ACD) of around 100 residues. Thirdly, the sHSPs share a compact $\beta$-sheet sandwich structure, which can dimerize creating the building block of the large oligomers [Haslbeck, 2015]. These features of the sHSP structure are based on analysis of the crystal structures of two sHSPs: one from wheat (Triticum aestivum), TaHsp16.9, and the other from an archaebacterium (Methanococcus jannaschii), MjHsp16.5 [Kim, 1998; van Montfort, 2001]. The sHSPs are ubiquitous proteins present in all living organisms. In plants, sHSPs are especially abundant and dissimilar, probably reflecting the need to rapidly adapt to changing environments (such as drought, temperature, light, chemical pollutants, and humidity). A comparative analysis of the SHSP sequences revealed that Arabidopsis has 19 sHSPs, rice (Oryza sativa) has 23, and poplar (Populus trichocarpa) has 36 [Waters, 2008b]. sHSPs have been grouped in 11 subfamilies based on nucleotidic sequence similarity, intracellular localization, and cross-reactivity in immunoblot analysis. From them, six subfamilies are cytoplasmic/nuclear localized ( $\mathrm{Cl}-\mathrm{CVI})$, and five subfamilies are localized in organelles. Among organellar sHSPs, some localize to the endoplasmic reticulum, to the peroxisome, to the chloroplast, and two subfamilies localize to the mitochondria (MTI and MTII) [Siddique, 2008; Bondino, 2012]. The high diversity of plant SHSPs had probably arisen as a consequence of the stressful conditions that plants suffer during their life cycle.

Plant SHSPs can be visualized in two processes, the stress response and the healthy development. During heat stress, most of the sHSPs are highly upregulated, which can assure the plant thermo-tolerance by protecting other proteins from irreversible denaturation. Additionally, the sHSPs were observed to respond to other stresses such as drought, salinity, cold, osmotic, and oxidative stress. Added to this, some plant sHSPs were observed at the typical development during embryogenesis, seed germination, pollen development, and fruit 
maturation [Wang, 2004b; Sun, 2005]. Several members of the sHSP family have been associated with membranes suggesting that these proteins are probably interacting with specific lipids, helping to control the physical state, the bilayer stability and the integrity of the membranes. It has been suggested that under stressful conditions, sHSPs and other HSPs may contribute to the membrane quality control and the maintenance of membrane integrity [Nakamoto, 2007]. Under normal growth conditions, sHSPs are generally undetectable in vegetative tissues but can be highly induced by environmental stresses and developmental stimuli.

\subsubsection{Structure of sHSPs.}

The primary amino acid sequence of sHSP comprises a variable region at the N-terminal, a more conserved region at the C-terminal, often referred to as the ACD domain or the HSP20 domain, and a C-terminal extension. The ACD is a region, highly conserved at the C-terminal, delimited from the $\beta 2$-strand to the $\beta 9$-strand. It is the core structure of the protein and is formed by two regions, $\mathrm{CRI}$ and $\mathrm{CRII}$ that form a sandwich of two pleated-sheets and are separated by a hydrophilic domain of variable length [Waters, 1996; Sun, 2002]. The ACD differentiates sHSPs from other small proteins induced by heat [Scharf, 2001]. The sHSPs that localize in particular cellular organelles, as most organellar proteins, have $\mathrm{N}$-terminal transit peptide or signal sequences required to direct the SHSP to the proper cellular compartment. The length and sequence of the C-terminal extension are highly variable, and may also contain amino acid motifs addressing the specific organelle [Waters, 2013].

One particular feature of most sHSPs is their capability to form oligomers, which has been considered essential for their function. The majority of sHSPs form large ensembles usually ranging from 12 to more than 32 subunits, although some dimers have also been described [Basha, 2013]. These oligomers are often polydisperse indicating that several oligomeric states exist [Baldwin, 2011]. The regulation of the chaperone activity of sHSPs correlates with the ability to find an equilibrium between different oligomer populations [Basha, 2012]. The oligomeric sHSPs are all assembled from a fundamental dimeric structure which is the basic building block and the active form of the protein. The dimer is stabilized by the formation of a $\beta$-sheet between the $\beta 6$-strand of one subunit and the $\beta 2$-strand of the 4 -pleated $\beta$-sheet of the other subunit. It is postulated that these three different regions existing in the protein contribute to SHSP oligomerization. While the ACD constitutes the basic dimeric building block, both flanking regions contribute to the assembly process. In particular, the C-terminal extension contains a conserved I-x-I motif that participates in the assembly of SHSP dimers into oligomers [Haslbeck, 2015]. 
Plant sHSPs can also arrange into heat shock granules which are approximately $40 \mathrm{~nm}$ in diameter. When heat stress last for long-term, unfolded proteins bound to sHSP oligomers accumulate in the cytoplasm, surpassing the refolding capacity of the Hsp70/Hsp40 system. These complexes of denatured proteins-sHSP can then be transiently stored in heat shock granules that break down during the recovery period [Sun, 2002].

The ACD is an ancient and conserved domain found in almost all eukaryotes, bacteria, and archaea. The designation 'alpha-crystallin domain' derived from the eye lens alpha-crystallin protein, a chaperone that protects denatured proteins from aggregation, and prevents the formation of cataracts in the eyes of vertebrates [Horwitz, 1992]. More recently, it has been suggested that the ACD was incorporated into a large number of other proteins, usually called ACD-proteins [Scharf, 2001; Bondino, 2012]. Within this group, not all ACD-containing proteins have chaperone activity and work as SHSPs. For instance, two ACD-proteins have been shown to participate in the DNA demethylation and gene silencing in Arabidopsis [Qian, 2014; Zhao, 2014].

The first two high-resolution crystal structures of sHSPs, MjHsp16.5 from Methanococcus jannaschii and TaHsp16.9 from wheat have in several common features. Both structures are composed of a $\beta$-sandwich of two antiparallel sheets, and the building blocks of both oligomers are dimers. Analysis of both crystal structures showed that MjHsp16.5 is formed by a hollow spherical complex composed of 24 subunits whereas the wheat TaHsp16.9 assembles into a dodecameric second disk containing six ACDs structured in a trimer of dimers [Kim, 1998; van Montfort, 2001]. To date, ten new high-resolution sHSP crystal have been published, but none of these is from plants. Moreover, all available sHSP structures lack full information of the Nterminal region structure, suggesting that this region might be intrinsically disordered, at least partially, or it may contain structural elements which are highly dynamic and oscillatory causing different contacts in diverse positions within the oligomer [Basha, 2012; Patel, 2014]. It has been proposed that the SHSP sequence at the $\mathrm{N}$-terminal region participates in the binding to denatured proteins [Giese, 2005; Basha, 2006].

\subsection{2. sHSPs functions.}

The functioning mechanism of sHSPs in cell defense is still under debate. As previously mentioned, the synthesis and accumulation of sHSPs in response to adverse and stressful conditions has been correlated with stress tolerance. One current model postulates that under conditions of extreme stress, sHSPs provide an energy-independent mechanism to buffer the increase in non-native proteins. Based on this model, sHSPs were postulated to function together with other chaperones to avoid irreversible protein aggregations and also acting on 
protein aggregates to re-solubilize the protein components [Waters, 2013]. sHSPs accomplish their role as molecular chaperones by stabilizing initial unfolding intermediates of aggregation in an ATP-independent manner. Apart from showing activity independent from ATP, SHSPs show a high capacity for binding denatured substrates and can bind one substrate protein per sHSP subunit of equal molecular mass [McHaourab, 2009]. In vitro experiments have demonstrated that SHSPs are mainly useful in preventing thermal aggregation of well-known mitochondrial enzymes, such as citrate synthase, malate dehydrogenase and luciferase. sHSPs can bind partially unfolded proteins on their surface and keep them in a foldingcompetent state [Lee, 1997].

At the moment, the resolved structure of an sHSP-substrate complex is lacking. It has been proposed that in this complex, SHSPs are rearranged from a previously dissociated form of the sHSPs oligomers, probably dimers, which re-assemble to a new oligomeric form holding the bound substrate. Thus, sHSPs can function as a buffering system for binding partially unfolded proteins upon stress and preventing them from irreversible aggregation [Haslbeck, 2015]. In vitro experiments showed that the unfolded protein enclosed in the sHSP/substrate complexes could be unbound and refolded when extra ATP-dependent chaperones are present [Lee, 2000; Mogk, 2003; Lee, 2005].

\subsubsection{Regulation of sHSPs and their role in stress response.}

Most sHSPs are not noticed in vegetative tissues under normal growth conditions, but, as their name implies, they are quickly synthesized in response to heat stress. After the stress ceases, sHSPs are somewhat stable showing long half-lives, up to $50 \mathrm{~h}$, suggesting that they may also participate in the cell recovery as well [Sun, 2002]. Information of the sHSPs gene expression revealed that some of them are also produced in response to other abiotic and biotic stresses, and are expressed at specific developmental stages, indicating the existence of diverse patterns of gene expression for all the plant sHSP genes [Siddique, 2008; Waters, 2008b]. The specific functions of SHSPs are characterized by the intracellular localization, the expression at different stress situations or developmental stages in different cell types and the interactions with specific client proteins.

In sunflowers, the HaHsp17.6 (class $\mathrm{Cl}$ ) and HaHsp17.9 (class $\mathrm{CII}$ ) accumulated in response to osmotic stress. These two proteins were induced in stems and roots of water-stressed sunflowers, and their mRNA levels positively correlate with the dehydration degree [Almoguera, 1993]. In Arabidopsis, the cytosolic AtHsp17.7-CII expression was induced by heat and osmotic stress, and during seed development. However, no detectable protein was observed under osmotic stress, suggesting a stress-induced post-transcriptional regulation of 
At-HSP17.6A expression [Sun, 2001]. Previews reports have shown the expression of sHSPs in oxidative stress in tomato [Banzet, 1998] and Arabidopsis plants [Scarpeci, 2008b], and in response to drought, UV radiation and wounding [Siddique, 2008]. Cold stress can trigger the induction and accumulation of several sHSPs. The tobacco CaHSP26 protein plays an essential role in protecting the PSII by maintaining the antioxidative system and by increasing the fluidity of the thylakoid membrane during chilling stress [Li, 2012]. The induction of SHSPs has been associated with the acquisition of low-temperature tolerance in pre-treated banana fruit [He, 2012]. Also, there is a robust indication of the relationship between the accumulation of sHSPs and the protection against chilling injury in tomato fruit [Polenta, 2007]. In previews studies, tomato fruit in the mature green stage was pre-treated with a temperature of $38{ }^{\circ} \mathrm{C}$ before preserving them at low temperatures. A strong induction of HSP70 proteins and SHSPs was observed even at low temperature, leading to an enhance chilling tolerance of the fruit [Lurie, 1997; Sabehat, 1998]. Besides this, different sHSPs were found and correlated with the differential resistance to chilling conditions of two contrasting tomato genotypes [Page, 2010]. Also in tomato, transgenic plants overexpressing a chloroplast-localized sHSP showed stronger chilling tolerance [Wang, 2005]. In more recent work using tomato fruit of two tomato varieties, Minitomato and Micro-Tom, it was shown the induction of sHSPs during ripening in fruit from both varieties. However, in response to cold temperatures of $4 \stackrel{\circ}{ } \mathrm{C}$, accumulation of sHSPs was only observed in the more cold-tolerant Micro-Tom fruit, indicating the role of these sHSPs in the chilling tolerance [Ré, 2016].

In the absence of adverse environmental situations, synthesis of SHSPs in plants occurs during several developmental processes, such as embryogenesis, germination, pollen development, and fruit maturation. Some sHSPs are expressed during embryogenesis and seed maturation in pea seeds grown under non-stress conditions [De Rocher, 1994], Arabidopsis [Wehmeyer, 1996], Allium cepa, Crocus albiflorus and Solanum tuberosum [Lubaretz, 2002]. It has been reported the expression of sHSPs during pollen development and fruit maturation. The chloroplast sHSP (HSP21) of tomato has a protective role of photosystem II (PSII) from temperature-dependent oxidative stress but is also involved in the conversion of fruit chloroplasts to chromoplasts during ripening [Neta-Sharir, 2005]. In tobacco, different subsets of cytosolic class I and II SHSP were expressed during development at all stages of pollen grain formation, suggesting that specific SHSP genes may play precise roles in early and during later stages of pollen development [Volkov, 2005].

The expression and accumulation of SHSP are mainly regulated at the transcriptional level by the HSFs that act through the heat shock promoter element (HSE). HSEs share the consensus sequence of 5'-AGAAnnTTCT-3' and are located upstream of the TATA box of heat stressinducible genes [Scharf, 2001]. Plant HSFs consist of three conserved evolutionary classes, A, B, and C. In tomato, HsfA1a, HsfA2, and HsfB1 would participate in a regulatory network 
that promotes the expression of HS responsive genes. HsfA1a which is defined as a master regulator of HSR in tomato is constitutively expressed and regulates the expression of HsfA2 and HsfB1, while HsfA2 in thermotolerant cells is the crucial HSF. HsfA1a is known to be a nuclear retention factor and co-activator of HsfA2 contributing to form a HsfA1a-HsfA2 heterooligomeric complex. Class B HSFs would act as attenuators of class A HSFs, except for the HsfB1 that participates in a novel role as co-activator of class A HSFs and other transcription factors. In Arabidopsis, HsfA1a and HsfA1b may be necessary for the initial phase of heat response while HsfA2 may control the gene expression under prolonged stressing and recovery conditions [Kotak, 2007]. Under physiological conditions, various HSFs show differential expression in specific tissues and particular developmental processes. Under stress conditions, HsfA2 is exclusively expressed in vegetative tissues, and in the initial phases of pollen development [Sun, 2002].

\subsection{Genomic organization of sHSPs and bidirectional promoters (BDPs).}

In eukaryotic, promoter regions usually consist of the main region of about $50 \mathrm{bp}$, known as a core promoter, nearby the transcription initiation site, and numerous distal DNA regulatory elements to control the final efficiency of the transcription process [Novina, 1996]. The importance of promoters in plant biotechnology and functional genomics research comes from the critical role they exert as the regulator of gene expression, and for their excellent potential application in genetic engineering. Nowadays, new genomes are available each month thanks to the development of sequencing techniques, making promising studies at the genome-scale sequence, such as the gene structure prediction, the gene organization in the genome and the gene regulation.

Two neighboring genes can be located in the same or opposite strand, and in a divergent, convergent or parallel configuration. Bidirectional genes comprise two adjacent genes located divergently on opposite DNA strands. The sequence between the two transcription start sites (TSS) of the genes is generally considered to be a potential bidirectional promoter [Trinklein, 2004; Yang, 2011b]. In silico and experimental analysis of the publicly accessible complete eukaryotes genome sequences from human to plants showed that bidirectional promoters are especially abundant and that bidirectional genes tend to be expressed similarly [Herr, 2004; Trinklein, 2004; Williams, 2004; Wang, 2009; Xu, 2009]. In humans, the distance between the two TSS in a bidirectional promoter is considered to be under 1,000 bp [Adachi, 2002; Trinklein, 2004]. Plants, on the other hand, contain intergenic regions between two bidirectional TSS longer than $1 \mathrm{kbp}$ [Mitra, 2009]. In Arabidopsis, about $13.3 \%$ of genes are arranged in a bidirectional structure [Wang, 2009]. From them, 5 gene pairs encoding sHSPs or ACD 
containing proteins were found head-to-head oriented with an intergenic region of less than 500 bp (Bondino \& Valle, personal communication). One of these pairs is the At5g51440At5g51430 encoding a mitochondrial sHPS (At5g51440). This fact makes interesting the study of BDPs of gene pairs encoding ACD containing proteins, and their potential uses in biotechnology.

With different regulation and expression patterns, BDPs turn to be of much interest in plant research in recent years. One of the first studies reported in native bidirectional promoter was about the 955-bp intergenic region of CaTin1 and CaTin2 genes, which directed the expression of the two genes in response to tobacco mosaic virus (TMV) [Shin, 2003]. Later on, BDPs have been successfully cloned from other plants species such as Arabidopsis, rice and maize [Bondino, 2009; Liu, 2016; Wang, 2016]. Several in silico analysis in rice, Arabidopsis, and Populus have shown that bidirectional genes regulated by BDPs share similar characteristics, such as co-expression, functional association, and conserved arrangement [Krom, 2008; Dhadi, 2009; Wang, 2009]. Expression data of large-scale studies in Arabidopsis revealed that adjacent genes are co-expressed, and a model has been proposed in which the more extended the intergenic sequences, the less the chance of the transcribed gene to be epigenetically regulated by differential expression [Williams, 2004; Colinas, 2008]. In some examples, bidirectional genes displayed similar expression patterns. That is the case of the tissue-specific and light-inducible bidirectional promoter located between cab1 and cab2 genes [Mitra, 2009]. In other cases, different expression patterns and expression levels of the bidirectional genes have been reported [Bondino, 2009; Banerjee, 2013]. In rice, a vast intergenic region of 1.8 Kbp located between the OCPI2 and OCPI1 (two members of the chymotrypsin protease inhibitor gene family) has been demonstrated to act as a bidirectional promoter [Singh, 2009]. In more recent research, RNA-seq and cDNA microarray data were combined to discover potential BDP in the rice genome, and four candidates showed bidirectional expression activity in various tissues. Two novel cis-sequences were found to be overrepresented and conserved in the four BDPs that can be associated with their bidirectionality. Interestingly, the bidirectional arrangement of the four gene pairs was shown to be conserved in six gramineous plants [Wang, 2016]. A recent promoter::GUS transgene approach in Arabidopsis has uncovered that the intergenic region between the genes At1g71850 and At1g71860 is an asymmetric bidirectional promoter, which exhibits a different expression profile depending on the orientation. The authors defined three functional regions within the BDP which regulated the expression in one or the other direction [Liu, 2015]. Similarly, the intergenic region between the maize defensin-like protein genes Def1 and Def2 show polarity and asymmetric strengths, functioning as an asymmetric bidirectional promoter that is specific to embryo [Liu, 2016]. Although these and other reports have extensively studied plant BDPs, it is still matters of debate how the epigenetic mechanisms regulate the bidirectional transcription and co- 
expression of gene pairs in plants. In a recent work, DNase-seq, RNA-seq, ChIP-seq, and MNase-seq data were integrated, and the effect of physical DNase I hypersensitive site (DHS) positions on the transcription of rice BDPs was elucidated. The metadata analysis indicated that the relative position of a DHS to the TSS of bidirectional gene pairs influences the expression of the corresponding head-to-head oriented genes, when the distance of a DHS to the TSS is short, the expression level of the genes is high [Fang, 2016b]. In another study, also in rice, several unique chromatin features were found in the BDPs but not in unidirectional promoters, including the overrepresentation of active histone marks (H3K4ac, H4K12ac, $\mathrm{H} 4 \mathrm{~K} 16 \mathrm{ac}, \mathrm{H} 3 \mathrm{~K} 9 \mathrm{ac}$, and H3K27ac), canonical nucleosomes and the underrepresentation of the repressive mark H3K27me3. The research indicates that histone acetylation may play important roles in the regulation of gene pairs and that coordination between active and repressive marks may exist to form a distinctive chromatin structure to enhance the coregulation of bidirectional gene pairs [Fang, 2016a]. These results demonstrate that combined arrangements of chromatin structures, histone modifications, and DHSs, which comprise functional cis-elements for interaction with the transcriptional machinery, may play an essential role in the regulation of the bidirectional transcription or co-expression in BDPs.

Bidirectional promoters show greater potential for practical uses than unidirectional promoters in genetic improvement since they can direct the expression of two genes simultaneously, and hence help to save time in constructing expression vectors [Mitra, 2009; Banerjee, 2013; Kumar, 2015]. By activating the expression of several genes, bidirectional promoters represent an efficient way to save energy and regulate simultaneously multiple genes. BDPs can be used for co-expressing multi-gene traits, or regulating the co-expression of genes functioning in the same, similar or linked biological pathways, and create products containing proteins from two head-to-head linked genes in stoichiometric quantities, a fact that is of much relevance and biologically significant. Much still needs to be investigated to better understand the functionality of BDP and the complex transcriptional regulation of bidirectional genes.

\subsection{Mitochondrial small heat shock proteins.}

As it was previously mentioned, plants SHSP are especially abundant and diverse. In this regard, higher plants are exceptional compared to other eukaryotes because sHSPs are found mostly in all organellar compartments, apart from the cytosol. Only in Drosophila melanogaster and Toxoplasma gondii, organellar forms of sHSPs has been described to date [Morrow, 2000; de Miguel, 2005]. In plants, the nuclear multigene families show many genes encoding organellar sHSPs localized in mitochondria, chloroplasts, endoplasmic reticulum, and peroxisome [Bondino, 2012; Waters, 2013]. 
It was formerly reported that, under heat stress, plants accumulate a greater amount of mitochondrial sHSPs (sHSP-M) compared to HSP60 and HSP70 [Lenne, 1994], and that the accumulation of SHSP-M positively correlated with the increase in tolerance of mitochondria at high temperatures [Chou, 1989]. Another study showed that the mitochondrial sHSP protected $\mathrm{NADH}$ : ubiquinone oxidoreductase (complex I) during heat stress in apple fruit of Pyrus pumila (P. Mill.) K. Koch var. Mclntosh [Downs, 1998]. This evidence suggests that these mitochondrial proteins have a role in the adaptation of plants to heat stress. To date, the sHSP$\mathrm{M}$ has been investigated in rice [Mani, 2015], pea [Avelange-Macherel, 2015], tobacco [Kim, 2011], tomato [Liu, 1999], Arabidopsis [Waters, 2008a] and maize [Lund, 2001]. However, much about the function of mitochondria-localized sHSPs is still unknown.

In previous phylogenetic classifications of sHSPs in Arabidopsis, three members were predicted to be mitochondria-localized and were grouped into two different families, MTI and MTII [Siddique, 2008; Waters, 2013]. The sHSP23.5 and sHSP23.6 proteins were classified into the MTI family, while the sHSP26.5 belongs to the MTII family. In tomato, on the contrary, only one SHSP-M with a molecular weight of $23.8 \mathrm{kDa}$ has been so far, described [Sabehat, 1998]. The molecular chaperone function of sHSP23.8 was confirmed in vitro. The recombinant SHSP23.8 was able to promote the renaturation of denatured citrate synthase via chemicals and protected it from thermal inactivation [Shono, 2002]. A single copy of sHSP23.8 was found in the tomato genomic DNA by Southern-blot analysis, and it was reported the higher expression of $s H S P 23.8$ after heat treatment in tomatoes with a temperature threshold of $36{ }^{\circ} \mathrm{C}$ [Liu, 1999; Shono, 2002]. sHSP23.8 is probably regulated by a negative feedback mechanism where the transcription of this SHSPs is regulated negatively by the accumulation of the protein. During the primary period of high heat stress at $40{ }^{\circ} \mathrm{C}$, rapid synthesis of the sHSP23.8 transcripts results in a significant accumulation of sHSP23.8 protein. This accumulation in the initial period of the heat stress might be interpreted as a feedback signal to slow down the transcription rate [Liu, 1999]. The sHSP23.8 promoter was further characterized in transgenic tomato plants by using the b-glucuronidase (GUS) reporter gene system. Robust GUS staining was spotted in the roots, leaves, flowers, fruit and germinated seeds after heat shock. GUS activity was also detected under other stress conditions such as oxidative stress, low temperatures, $y$-rays, exogenous ABA and heavy metals, indicating that sHSP23.8 is also up-regulated in these conditions, besides high temperatures [Yi, 2006]. It has been demonstrated that SHSP23.8 provides thermotolerance in transformed tobacco plants with the tomato SHSP23.8 gene. Tobacco transgenic plants overexpressing SHSP23.8 showed high thermotolerance, while antisense plants were more susceptible to the heat treatment [Sanmiya, 2004]. In tomato, T0 and T1 overexpressing lines showed also increased thermotolerance under high temperature leading to the conclusion that sHSP23.8 is not just expressed by plants under heat-shock, but has a unique function in thermotolerance [Nautiyal, 
2005]. In a different report, cycles of high temperature $\left(37^{\circ} \mathrm{C}\right)$ and recovery at $21^{\circ} \mathrm{C}$ were used to evaluate the heat stress response and the photosynthetic activity of tomato Micro-Tom plants overexpressing the sHSP23.6-M from Arabidopsis. Plants with higher levels of sHSP23.6-M showed an increase in some parameters associated with the photosystem II activity and the net assimilation of $\mathrm{CO}_{2}$. The results suggest that in response to heat stress, the higher expression of SHSP23.6-M results in less damage to the photosynthetic system and that heat tolerance can be attributed, at least in part to these proteins [Hüther, 2013]. When the same mutant plants were exposed to flooding, MT-sHSP23.6 sense plants could maintain the connectivity and flow of energy between the units of the PSII during flooding and recovery, showing higher tolerance to the stress treatment. These data indicate that Arabidopsis sHSP23.6-M may play an important role not only in the heat but also in hypoxia stress in tomato [Hüther, 2016].

Furthermore, pre-treated tomato fruit with high temperature before being subjected to cold treatment showed the induction of SHSP23.8 after the heat-treatment, which persisted and prolonged the post-harvest life of fruit stored at low temperatures [Sabehat, 1996]. In a different approach, the chilling injury was investigated in tomato fruit cv. Micro-Tom which has been proposed to be a chilling-tolerant variety [Gonzalez, 2015]. Increased expression of the sHSP23.8 after cold treatment was observed in Micro-Tom fruit but not in Minitomato (a chilling-sensitive variety), what indicates that this protein may play central roles in the chilling tolerance of tomato fruit [Ré, 2016].

\subsection{Cold stress and chilling injury in plants.}

To understand how plants cope with chilling stress, it is essential to know the molecules and pathways involved in the chilling tolerance or sensitivity in tomato fruit. Chilling stress is important during storage at low temperature, which is an appropriate strategy to prolong the market life of many vegetables [Kader, 2003]. This practice drops the functioning of metabolic pathways and reduces pathogenic events, making exportation more favorable for long distance shipment and consequently, a more regulated supply of fruit in the market arises. However, the outcome of storing the plant products at low, critical temperatures is chilling injury $(\mathrm{Cl})$ that generates high economic losses. Sensitive to low temperatures are not only fruits, but vegetables, and ornamentals of tropical or subtropical origin. Certain horticultural crops of temperate origin are also susceptible to chilling injury such as tomato. These temperate crops, in general, have lower threshold temperatures, around $5^{\circ} \mathrm{C}$ [Wang, 2004a]. $\mathrm{Cl}$ is the set of physiological alterations and dysfunction that appear during the exposure of plants to low temperatures above the freezing point (between $0^{\circ} \mathrm{C}$ and $15^{\circ} \mathrm{C}$ ) [Lyons, 1973]. Many 
physiological and biochemical consequences of chilling injury have been widely described. However, the molecular mechanisms behind the generation and tolerance to chilling stress in fruit remain to be understood.

Under chilling temperatures, tissues deteriorate because they are incapable of carrying on regular metabolic processes. Chilling-sensitive species respond to chilling stress by altering numerous processes at physiological and biochemical levels and thus, the cells start to function anomaly [Wang, 2004a]. These disorders constitute one of the main limitations of the commercial life of many fruit and vegetable products. Reducing the effects of $\mathrm{Cl}$ would lead to greater availability of food, smaller areas of land needed for cultivation and the possibility of exporting to new international markets.

\subsubsection{Symptoms of $\mathrm{Cl}$.}

In fruit, symptoms of $\mathrm{Cl}$ are diverse and depend mainly on the cultivar, the temperature and time of exposure, the degree of maturity, the climatic characteristics of the growing area and the temperatures prior harvesting. Other factors can also affect the postharvest life of cold stored fruits and the development of $\mathrm{Cl}$, such as the relative humidity of the environment and the presence of ethylene in the storage atmosphere. Common symptoms of $\mathrm{Cl}$ in fruits include depressions and surface wounds, pitting, internal colour alterations, water-soaking of the tissue, inability to typically ripen which causes lack of uniformity in the surface and pulp colour, higher susceptibility to microorganisms and pathogens and loss of water [Lyons, 1973; Wang, 2010]. Besides, there is a decrease in the sweetness, aroma and characteristic flavor of the fruit, caused by a metabolic imbalance [Maul, 2000]. Surface pitting is one of the most common symptoms in many fruits and vegetables such as citrus fruits, cucumbers, eggplant, melons, and sweet potatoes. Failure to ripen was observed in chilled avocados, bananas, mangos, melons, and tomatoes. Also, it is usually the internal discoloration in avocados, pineapples, and sweet potatoes [Wang, 2010]. This diversity of $\mathrm{Cl}$ symptoms of tropical and subtropical fruits and vegetables suggests multiple responses to low temperature. Products that are stored at chilling temperatures do not show $\mathrm{Cl}$ symptoms when remaining in low temperatures. In some cases, these characteristics can develop progressively during the exposure to low temperatures but usually develop and become evident in a short time after products are transferred to room temperature [Malacrida, 2006; Gonzalez, 2015]. 


\subsubsection{The theories of $\mathrm{Cl}$.}

In previews reports, some molecular mechanisms have been suggested to accommodate the physiological and biochemical changes associated with Cl [Lukatkin, 2012]. One of the most discussed theory proposed that chilling sensitivity can be explained by a phase transition of cell membranes that occurs at low temperatures and converts the membranes from a flexible liquid-crystal state into a rigid state of gel-solid. These changes trigger severe alterations at the membrane and enzymatic membrane-binding system that culminate with adverse events such as membrane damage, loss of electrolytes, failure of respiration and the production of toxic compounds. This phase transition in even a small proportion of membrane lipids results in the formation of solid domains that can cause cell damage. When the exposure to cold is brief, the effect may be transient, and the cell survives. However, when stress is prolonged, necrosis and cell death occur [Lyons, 1973]. This hypothesis that proposed the phase transition of membrane lipids as the primary cause of $\mathrm{Cl}$ has been discredited. The membrane changes do not happen instantly after the start of chilling and are more likely to be less critical syndromes. According to the phase transition hypothesis, the rise in membrane permeability occurring because of the low-temperature condition (causing membranes leakage) should be fast, recorded immediately (minute scale) after placing the tissue at chilling temperatures. Nevertheless, this is not observed, and frequently passive permeability is not augmented. However, there is no doubt that the physical properties of membranes are crucial for cell homeostasis, and that they may be especially deteriorated during the cellular response to chilling stress [Lukatkin, 2012].

Another theory of $\mathrm{Cl}$ is based on the metabolic disorder that occurs in cold temperature. In this case, cell death takes place due to the prevalence of disintegration over synthesis, and to the dissociation of enzymes and other proteins, which would result in changes in enzymatic kinetics and/or in structural changes of specific proteins such as tubulins [Graham, 1982]. Low temperatures would induce a decrease of hydrophobic binding forces, altering protein-protein and lipid-protein interactions. The overall disruption may cause the malfunction of soluble enzymes, dissociation of subunits and unfolding of the proteins [Parkin, 1989].

In other reports, special attention has been drawn to two different hypotheses to explain the induction of $\mathrm{Cl}$, one to a fast uprise in the free cytosolic Ca2+ level $\left([\mathrm{Ca} 2+]_{\mathrm{cyt}}\right)$ and the other to the occurrence of oxidative stress upon chilling [Minorsky, 1985; Prasad, 1994]. The quick rise in $[\mathrm{Ca} 2+]_{\text {cyt }}$ due to chilling, may work for as the primary physiological indication of cold exposure. Changes in $[\mathrm{Ca} 2+]_{\mathrm{cyt}}$ activate cascade reactions in the cell, which leads to many disorders at all levels of organization. When changes in the compartmentation of calcium in the chilled plants occurred, they directed to an increase in [Ca2+] $]_{\text {cyt, }}$ end cytoplasmic streaming and disturb the subcellular structures. For proposing the calcium hypothesis, it was taking into 
account the oxidative stress during chilling that plays a crucial function in the transduction of the chilling signal. It was shown that $[\mathrm{Ca} 2+]_{\text {cyt }}$ changes and oxidative stress are intimately connected under chilling conditions. The increase of free radicals and ROS in chilling produce substantial alteration to membrane lipids and other cellular components [Suzuki, 2006].

\subsection{3. $\mathrm{Cl}$ in tomato fruit.}

The effect of cold storage in the ripening process of tomato fruit has been the subject of numerous studies [Rugkong, 2011; Ré, 2012; Tao, 2014; Cruz-Mendívil, 2015; Gonzalez, 2015]. The global transcriptomic analysis in cold-stored tomato fruit revealed the downregulation of genes involved in color development, including phytoenesynthase 1 and carotenoid isomerase, and in genes encoding the cell wall modifying proteins polygalacturonase, pectin esterase1, $\beta$-galactosidase, expansin1, and xyloglucan endotransglucosylase-hydrolase 5 [Rugkong, 2011]. Besides the reduction of color-related genes, the alteration described in the coloration of tomato fruit was explained by the inability to accumulate lycopene [Watkins, 1990; Malacrida, 2006]. To understand the mechanisms responsible for the tolerance to $\mathrm{Cl}$ observed in Micro-Tom fruit, a recent study combined metabolomics and transcriptomics data of Micro-Tom fruit after chilling storage and the changes in the overall metabolome including primary metabolites, carotenoids, lycopene, soluble antioxidants, tocopherols, and tocotrienols, and transcriptome after chilling were investigated. The results showed alterations in the metabolism of reserves, fermentation and amino acids mobilization and photosynthesis, and the induction of defense mechanisms. After removing the fruit from refrigeration, the photosynthetic activities and the transcripts related showed a minor recovery. Transcriptional up-regulation of genes coding for proteins that accumulate in response to low temperatures, along with genes encoding antioxidant enzymes and SHSP was also observed. In addition to this, it was found a robust up-regulation of AOX gene transcription and a rise in pyruvate content which is a positive effector of AOX. Moreover, the level of ethanol and several genes involved in fermentation processes increased after chilling indicating a fermentative physiological response of the fruit [Gonzalez, 2019]. Proteomics studies have shown that the levels of proteins related to maturation of the fruit decreased with cold storage, while proteins related to the stress response increased [Page, 2010]. It has been shown that storage at low temperatures also decreases respiration and ethylene synthesis, what could modify the expression of many genes that explain the following symptoms, although it has also been seen that ethylene is not essential for the appearance of symptoms of Cl [Luengwilai, 2010]. 


\subsubsection{Oxidative stress and $\mathrm{Cl}$.}

All aerobic organisms require oxygen as an essential element of their metabolism. The oxygenic environment may, however, involve potential hazards for the cells. During normal metabolism, intermediary products of the reduction of oxygen that show a high reactivity are produced in various subcellular compartments. These intermediates are called ROS [Mittler, 2002]. ROS species include superoxide anion $\left(\mathrm{O}^{-}\right)$, hydrogen peroxide $\left(\mathrm{H}_{2} \mathrm{O}_{2}\right)$, hydroxyl radical $(\cdot \mathrm{OH})$ and singlet oxygen $\left({ }^{1} \mathrm{O} 2\right)$ produced by physical excitation of $\mathrm{O}_{2}$. In the course of evolution, plants have developed an intricate and efficient network to remove and mitigate the toxicity of ROS and started to use some of these toxic molecules as mediators in signal transduction [Mittler, 2004; Bailey-Serres, 2006]. It has been proposed that ROS action has a double effect in plants, acting as toxic compounds, and at the same time as crucial regulators of numerous pathways related with critical biological processes such as growth, cell cycle, programmed cell death, hormonal signalling, response to biotic and abiotic stress, and development [Mittler, 2004]. To maintain the dual role, there must be a delicate balance between the production and the removal of ROS. The term "oxidative stress" is usually used to describe situations in which the generation of ROS surpasses the cell capacity of keeping the redox homeostasis [Gill, 2010].

In plants, the production of ROS occurs in the apoplast and several subcellular compartments such as peroxisomes, chloroplasts, mitochondria and the nucleus [Toivonen, 2004]. Although chloroplasts are usually the primary site of ROS production in plants, in post-harvest fruit other organelles may become important sites for the generation of ROS. Due to their high oxygen consumption, mitochondria are the leading producers of ROS in non-photosynthetic tissues [Hodges, 2003].

ROS molecules can react producing lipid peroxidation, polysaccharides and protein degradation, and disruption of DNA molecules. When the intracellular ROS concentration increases uncontrollably, irreversible damage is produced leading to cell death [Gill, 2010]. A model has been proposed to describe the function of ROS signaling pathways in the chilling stress response. According to this model, $\mathrm{Cl}$ probably start from a membrane receptor, still unknown, that would sense the change in the temperature and activate an NADPH membrane oxidase, causing a controlled increase in the levels of ROS, which would function as a regulatory signal for the expression of responsive genes [Einset, 2007].

The oxidative stress produced during storage at low temperatures is one of the main factors that contribute to the generation of $\mathrm{Cl}$. The loss of integrity and fluidity of membranes may affect the protein functions either by the direct action of ROS, by alteration of the activity due to an unfavorable lipid context or by the conjunction of both situations. In this way, the alteration and dysfunction of critical enzymes lead to a metabolic imbalance that manifests at the tissue 
level as a visible symptom of Cl [Prasad, 1994; Shewfelt, 2000]. Many studies have linked the occurrence of post-harvest $\mathrm{Cl}$ after storage of fruit at low temperatures with the generation of oxidative stress, and it has been postulated that the antioxidant capacity of the cell may play an essential role in the prevention of $\mathrm{Cl}$ symptoms. It has been shown that cold-tolerant species produce fewer ROSs and more antioxidant compounds [Sala, 1999; Malacrida, 2006]. 


\section{Aims of this study}

Under the theoretical framework developed, the general objective of this thesis is to study the regulation of the expression of genes encoding putative SHSPs divergently orientated in the genome and their functionality under environmental stress in Arabidopsis thaliana. In addition, the aim of this work is to investigate the role of mitochondrial sHSPs in A. thaliana and Solanum lycopersicum.

The specific aims of this work are:

2.1. To study the expression of four genes encoding proteins with $A C D$ and potential sHSPs with head-to-head orientation in the Arabidopsis genome, and the functionality of the intergenic regions, which are putative bidirectional promoters, under abiotic stress.

2.2. To analyze the expression of genes encoding mitochondrial SHSPs in Arabidopsis and their in vivo functionality by generating transgenic Arabidopsis plants with altered expression (loss-of-function).

2.3. To study the role of the mitochondrial SHSP23.8 in conferring chilling tolerance in tomato fruit by using transgenic tomato plants with reduced level of this protein. 


\section{Material and Methods}

\subsection{Material}

\subsubsection{Chemicals}

All chemicals and solvents were supplied by Sigma-Aldrich (St. Louis, USA), Merck KGaA (Darmstadt, Germany) and Carl Roth \& Co. (Karlsruhe, Germany) unless stated otherwise. Solvents in LC-MS-grade like methanol, ethanol, and acetonitrile were obtained from Thermo Fisher Scientific (Waltham, USA).

\subsubsection{Kits, enzymes and commercial reagents}

Table 3.1. Molecular biological kits, enzymes and size markers used in this study.

\begin{tabular}{|ll|}
\hline Kit, enzyme or marker & Supplier \\
\hline CloneJETPCR Cloning Kit & Thermo Fisher Scientific (Waltham, USA) \\
DNasel & Thermo Fisher Scientific (Waltham, USA) \\
GenElute Plasmid Miniprep Kit & Sigma-Aldrich Co. (St. Louis, USA) \\
Gene-Ruler 1kb DNA Ladder & Thermo Fisher Scientific (Waltham, USA) \\
GoTaq Polymerase & Promega Corporation (Madison, USA) \\
RedTaq Polymerase ready mix & Sigma-Aldrich Co. (St. Louis, USA) \\
Nucleospin Gel and PCR Clean-up & Macherey-Nagel (Dueren, Germany) \\
Phusion High-Fidelity Polymerase & Thermo Fisher Scientific (Waltham, USA) \\
Restriction endonuclease enzymes & Thermo Fisher Scientific (Waltham, USA) \\
RevertAid H Minus reverse transcriptase & Thermo Fisher Scientific, Waltham, USA \\
T4-DNA-Ligase & Thermo Fisher Scientific (Waltham, USA) \\
Takyon No ROX SYBR Mastermix blue & Kaneka Eurogentec S.A. (Seraing, \\
dTTP & Belgium) \\
TLC Silica gel 60 & Merck KGaA (Darmstadt, Germany) \\
\hline
\end{tabular}

\subsubsection{Media}

\subsubsection{Media used for Escherichia coli growth}

The medium used for cultivation of Escherichia coli was Lysogeny Broth (LB) medium. The medium was autoclaved $20 \mathrm{~min}$ at $120^{\circ} \mathrm{C}$. For plates, $1.5 \%(\mathrm{w} / \mathrm{v})$ agar was added to the LB medium before autoclaving to obtain solid LB. For plates preparation and the addition of specific antibiotics, the medium was chilled to about $60^{\circ} \mathrm{C}$ and used under sterile conditions. 
Table 3.2. Lysogeny Broth (LB) medium [Bertani, 1951].

\begin{tabular}{|ll|}
\hline Component & Amounts \\
\hline Tryptone & $10 \mathrm{~g} / \mathrm{L}$ \\
Yeast extract & $5 \mathrm{~g} / \mathrm{L}$ \\
$\mathrm{NaCl}$ & $5 \mathrm{~g} / \mathrm{L}$ \\
Water & Add to $1 \mathrm{~L}$ \\
\hline
\end{tabular}

\subsubsection{Media for plant cultivation}

Arabidopsis thaliana plants were cultivated under sterile conditions on plates with $1 / 2$ Murashige Skoog (1/2 MS) medium [Murashige, 1962]. In some experiments, liquid $1 \frac{1}{2}$ MS medium without agar was used.

Table 3.3. $1 / 2$ MS medium

\begin{tabular}{|ll|}
\hline Component & Amounts \\
\hline Murashige Skoog powder & $2.2 \mathrm{~g} / \mathrm{L}$ \\
Agar & $8 \mathrm{~g} / \mathrm{L}$ (solid medium) \\
Water & Add to $1 \mathrm{~L}$ \\
\hline
\end{tabular}

For Solanum lycopersicum cultivation and in vitro propagation under sterile conditions, the following media were used:

Table 3.4. Germination media (GM)

\begin{tabular}{|ll|}
\hline Component & Amounts \\
\hline Murashige Skoog (MS) \& Gamborg vitamins (B5) (Duchefa) & $2.2 \mathrm{~g} / \mathrm{L}$ \\
Sucrose & $15 \mathrm{~g} / \mathrm{L}$ \\
Agar & $9 \mathrm{~g} / \mathrm{L}$ \\
Water & Add to $1 \mathrm{~L}$ \\
pH: 6 & \\
\hline
\end{tabular}

Table 3.5. Co - Cultivation media (CM)

\begin{tabular}{|ll|}
\hline Component & Amounts \\
\hline Murashige Skoog (MS) \& Gamborg vitamins (B5) (Duchefa) & $4.4 \mathrm{~g} / \mathrm{L}$ \\
Sucrose & $30 \mathrm{~g} / \mathrm{L}$ \\
Tiamine HCL & $1 \mathrm{mg} / \mathrm{L}$ \\
2,4-Dichlorophenoxyacetic acid (2,4 D) & $1 \mathrm{mg} / \mathrm{L}$ \\
Kinetin & $0.2 \mathrm{mg} / \mathrm{L}$ \\
Agar & $9 \mathrm{~g} / \mathrm{L}$ \\
Water & Add to $1 \mathrm{~L}$ \\
pH: 6 & \\
\hline
\end{tabular}

Table 3.6. Shoot induction media (SIM)

\begin{tabular}{|ll|}
\hline Component & Amounts \\
\hline Murashige Skoog (MS) \& Gamborg vitamins (B5) (Duchefa) & $4.4 \mathrm{~g} / \mathrm{L}$ \\
Sucrose & $30 \mathrm{~g} / \mathrm{L}$ \\
Indole-3-acetic acid (IAA) & $0.175 \mathrm{mg} / \mathrm{L}$ \\
6-Benzylaminopurine (BAP) & $2.25 \mathrm{mg} / \mathrm{L}$ \\
Ticarcillin/clavulanic acid & $250 \mathrm{mg} / \mathrm{L}$ \\
Cefotaxime & $125 \mathrm{mg} / \mathrm{L}$ \\
\hline
\end{tabular}




\begin{tabular}{|ll|}
\hline Kanamycin & $40 \mathrm{mg} / \mathrm{L}$ \\
Agar & $9 \mathrm{~g} / \mathrm{L}$ \\
Water & Add to $1 \mathrm{~L}$ \\
$\mathrm{pH}: 6$ & \\
\hline
\end{tabular}

Table 3.7. Rooting media (RM)

\begin{tabular}{|ll|}
\hline Component & Amounts \\
\hline Murashige Skoog (MS) \& Gamborg vitamins (B5) (Duchefa) & $2.2 \mathrm{~g} / \mathrm{L}$ \\
Sucrose & $15 \mathrm{~g} / \mathrm{L}$ \\
Agar & $9 \mathrm{~g} / \mathrm{L}$ \\
1-Naphthaleneacetic acid [Rodriguez, 2010] & $0.2 \mathrm{mg} / \mathrm{L}$ \\
Water & Add to $1 \mathrm{~L}$ \\
pH: 6 & \\
\hline
\end{tabular}

\subsubsection{Antibiotics}

Different antibiotics were added to the LB or MS $1 / 2$ media and used for bacteria or plant selection. Given are the final concentrations of the antibiotics.

Table 3.8. Antibiotics

\begin{tabular}{|ll|}
\hline Antibiotic & Concentration $(\boldsymbol{\mu g} / \mathbf{m L})$ \\
\hline Ampicillin & 100 \\
Carbenicillin & 100 \\
Cefotaxime & 125 \\
Gentamicin & 25 \\
Kanamycin & $25-40$ (for plants), 100 (for bacteria) \\
Rifampicin & 25 \\
Spectinomycin & 90 \\
Ticarcillin/clavulanic acid & 250 \\
\hline
\end{tabular}

\subsubsection{Sodium dodecyl sulfate-polyacrylamide gel electrophoresis (SDS-PAGE)}

Table 3.9. SDS-PAGE

\begin{tabular}{|lll|}
\hline Stocks & Staking gel & Separative gel \\
\hline $\begin{array}{l}\text { Final concentration } \\
\text { of Acrylamide/Bis-acrylamide }\end{array}$ & $4 \%$ & $12 \%$ \\
$\begin{array}{l}\text { Acrylamide/Bis-Acrylamide } \\
\text { (Stock concentration = } 30 \%)\end{array}$ & $1.3 \mathrm{~mL}$ & $6.4 \mathrm{~mL}$ \\
$1.5 \mathrm{M}$ TRIS pH 8.8 & - & $4.00 \mathrm{~mL}$ \\
$0.5 \mathrm{M}$ TRIS pH 6.8 & $2.50 \mathrm{~mL}$ & - \\
Water & $6.1 \mathrm{~mL}$ & $5.5 \mathrm{~mL}$ \\
$25 \%$ Ammonium persulfate & $0.040 \mathrm{~mL}$ & $0.064 \mathrm{~mL}$ \\
TEMED & $0.010 \mathrm{~mL}$ & $0.016 \mathrm{~mL}$ \\
Total volume & $10 \mathrm{~mL}$ & $16 \mathrm{~mL}$ \\
\hline
\end{tabular}




\subsubsection{Buffers}

Table 3.10. SDS running buffer, for $2.5 \mathrm{~L}$

\begin{tabular}{|ll|}
\hline Component & Amounts \\
\hline TRIS/HCl, pH 8 & $3 \mathrm{~g}$ \\
Glycerol & $14.4 \mathrm{~mL}$ \\
Sodium dodecyl sulfate (SDS) & $1.0 \mathrm{~g}$ \\
\hline
\end{tabular}

Table 3.11. TAE buffer

\begin{tabular}{|ll|}
\hline Component & Final concentration \\
\hline TRIS/HCl, pH 7 & $40 \mathrm{mM}$ \\
Acetic acid & $20 \mathrm{mM}$ \\
Ethylenediaminetetraacetic acid (EDTA) & $1 \mathrm{mM}$ \\
\hline
\end{tabular}

Table 3.12. 2x Laemmli sample buffer

\begin{tabular}{|ll|}
\hline Component & Final concentration \\
\hline Tris/HCl, pH 6.8 & $100 \mathrm{mM}$ \\
DTT & $200 \mathrm{mM}$ \\
SDS & $4 \%(\mathrm{w} / \mathrm{v})$ \\
Bromophenol blue & $0.002 \%(\mathrm{w} / \mathrm{v})$ \\
Glycerol & $40 \%(\mathrm{w} / \mathrm{v})$ \\
\hline
\end{tabular}

Table 3.13. 6x loading dye

\begin{tabular}{|ll|}
\hline Component & Final concentration \\
\hline Tris & $40 \mathrm{mM}$ \\
EDTA & $2 \mathrm{mM}$ \\
Glycerol & $50 \%(\mathrm{v} / \mathrm{v})$ \\
Bromophenol blue & $0.4 \%(\mathrm{w} / \mathrm{v})$ \\
\hline
\end{tabular}

Table 3.14. Cetyltrimethylammonium bromide (CTAB) extraction buffer

\begin{tabular}{|ll|}
\hline Component & Amounts \\
\hline CTAB & $2 \%(\mathrm{w} / \mathrm{v})$ \\
Tris/HCl pH 8.0 & $100 \mathrm{mM}$ \\
EDTA & $20 \mathrm{mM}$ \\
$\mathrm{NaCl}$ & $1.4 \mathrm{M}$ \\
\hline
\end{tabular}

\subsubsection{Plasmids}

Vectors are shown with their relevant features in Table 3.15.

Table 3.15. Plasmids

\begin{tabular}{|lll|}
\hline Plasmid & Selection Marker & Reference \\
\hline pjet1.2/blunt & Ampicillin & Thermo Fisher Scientific (Waltham, USA) \\
Zero blunt & Kanamycin & Promega Corporation (Madison, USA) \\
MR202 Binary Vector & Spectinomycin/Kanamycin & Dr. Martin de Ré (IBR-UNR-CONICET) \\
GF9 Binary Vector & Spectinomycin/Kanamycin & Dr. Gisela Ferraro (IBR-UNR-CONICET) \\
pCHF3 Binary Vector & Spectinomycin/Kanamycin & Dr. Javier Palatnik (IBR-UNR-CONICET) \\
NB147 & Kanamycin & Dr. Javier Palatnik (IBR-UNR-CONICET) \\
\hline
\end{tabular}




\subsubsection{Bacterial strains}

Escherichia coli Top 10 (Agilent Technologies, Santa Clara, USA).

Escherichia coli DH5- $\alpha$ (New England Biolabs, Ipswich, USA).

Agrobacterium tumefaciens GV2260 and GV3101 pMP90.

\subsubsection{Plant material}

Plants from Arabidopsis thaliana ecotype Col-0 were used as a control in all experiments and for plant transformation. Seeds were obtained from Arabidopsis Biological Resource Center (ABRC, https://www.arabidopsis.org). For tomato experiments, seeds of the tomato cv. MicroTom were used as a control and for the generation of transgenic plants. Seeds were provided by Gulf Coast Research and Education Center, University of Florida, USA.

\subsubsection{Oligonucleotides}

Table 3.16. Oligonucleotides

\begin{tabular}{|c|c|c|c|}
\hline Name & Sequence (5'to $3^{\prime}$ ) & Used in & $\begin{array}{l}\text { Restriction } \\
\text { sites }\end{array}$ \\
\hline At4g660 prom-F & GGTACCGGCGTTGTGTGGTGGTA & Cloning & Kpnl \\
\hline At4g660 prom-R & GGATCCTGATTCTATCTTTACCAAG & Cloning & BamHI \\
\hline At4g650 prom-F & GGTACCTGATTCTATCTTTACCAAG & Cloning & Kpnl \\
\hline At4g650 prom-R & GGATCCGGCGTTGTGTGGTGGTA & Cloning & BamHI \\
\hline At5g440 prom-F & GGTACCCCTTGATCCGATCACCCG & Cloning & Kpnl \\
\hline At5g440 prom-R & GGATCCTTTTGGAAAGAGAAGAAG & Cloning & BamHI \\
\hline At5g430 prom-F & GGTACCTTTTGGAAAGAGAAGAAG & Cloning & Kpnl \\
\hline At5g430 prom-R & GGATCCCCTTGATCCGATCACCCG & Cloning & BamHI \\
\hline At2g500 prom-F & GGTACCGGCCGATCTTTGTGTTCG & Cloning & Kpnl \\
\hline At2g500 prom-R & GGATCCTGATGATTGATTTCAAAC & Cloning & BamHI \\
\hline At2g490 prom-F & GGTACCTGATGATTGATTTCAAAC & Cloning & Kpnl \\
\hline At2g490 prom-R & GGATCCGGCCGATCTTTGTGTTCG & Cloning & BamHI \\
\hline At1g470 prom-F & GGTACCCTCTGATTGATTCGATCG & Cloning & Kpnl \\
\hline At1g470 prom-R & GGATCCCAATAATCTGAACTCACC & Cloning & BamHI \\
\hline At1g460 prom-F & GGTACCCAATAATCTGAACTCACC & Cloning & Kpnl \\
\hline At1g460 prom-R & GGATCCCTCTGATTGATTCGATCG & Cloning & BamHI \\
\hline At1g850 prom-F & GGTACCATCCTAAACACACAACAC & Cloning & Kpnl \\
\hline At1g850 prom-R & GGATCCCGGAGACGATGTTTCAGA & Cloning & BamHI \\
\hline At1g840 prom-F & GGTACCCGGAGACGATGTTTCAGA & Cloning & Kpnl \\
\hline At1g840 prom-R & GGATCCATCCTAAACACACAACAC & Cloning & BamHI \\
\hline At4g200 prom-F & CTGGTACCTTTCTTCTTTAATATATGACG & Cloning & Kpnl \\
\hline At4g200 prom-R & CTGGATCCTTGTAGAGAAACAGGAAGC & Cloning & BamHI \\
\hline At1g560 prom-F & GGTACCTTAAAAAATGTATATGAGCA & Cloning & Kpnl \\
\hline At1g560 prom-R & GGATCCTGTTTTCAAATCGGTAAATTTC- & Cloning & BamHI \\
\hline amiRNA200A & & Cloning & \\
\hline amiRNA200B & $\begin{array}{l}\text { AGGAGACGGCGTTGATTTATATCAAAG- } \\
\text { AGAATCAATGAT }\end{array}$ & Cloning & \\
\hline amiRNA200C & $\begin{array}{l}\text { AATAAATGAACGCCGTCTTCTCTACATA- } \\
\text { TATATTCCTA }\end{array}$ & Cloning & \\
\hline amiRNA200D & $\begin{array}{l}\text { TATAAATCAACGCCGTCTCCTCTCTCTT- } \\
\text { TTGTATTCC }\end{array}$ & Cloning & \\
\hline amiRNA440A & $\begin{array}{l}\text { CAAGTAAGTGAAAACCCTTTTTCACAG- } \\
\text { GTCGTGATATGA }\end{array}$ & Cloning & \\
\hline
\end{tabular}




\begin{tabular}{|c|c|c|c|}
\hline amiRNA440B & $\begin{array}{l}\text { CAGGTAAGCGAAATCCCTTTATCAAA- } \\
\text { GAGAATCAATGAT }\end{array}$ & Cloning & \\
\hline amiRNA440C & $\begin{array}{l}\text { AAAAGGGTTTTCACTTACTTGCTACA- } \\
\text { TATATATTCCTA }\end{array}$ & Cloning & \\
\hline amiRNA440D & $\begin{array}{l}\text { TAAAGGGATTTCGCTTACCTGCTCTC- } \\
\text { TTTTGTATTCC }\end{array}$ & Cloning & \\
\hline amiRNA560A & $\begin{array}{l}\text { TCATGAATTCATTGCAACTATTCACA- } \\
\text { GGTCGTGATATGA }\end{array}$ & Cloning & \\
\hline amiRNA560B & $\begin{array}{l}\text { TCGTGAATCCATTCCAACTAATCAAA- } \\
\text { GAGAATCAATGAT }\end{array}$ & Cloning & \\
\hline amiRNA560C & $\begin{array}{l}\text { ATAGTTGCAATGAATTCATGACTACA- } \\
\text { TATATATTCCTA }\end{array}$ & Cloning & \\
\hline amiRNA560D & $\begin{array}{l}\text { TTAGTTGGAATGGATTCACGACTCTC- } \\
\text { TTTTGTATTCC }\end{array}$ & Cloning & \\
\hline amiRNA440-200A & $\begin{array}{l}\text { CTAGTATCGGCTAGTCGTGGTTCACA- } \\
\text { GGTCGTGATATGA }\end{array}$ & Cloning & \\
\hline amiRNA440-200B & $\begin{array}{l}\text { CTGGTATCAGCTACTCGTGGATCAAA- } \\
\text { GAGAATCAATGAT }\end{array}$ & Cloning & \\
\hline amiRNA440-200C & $\begin{array}{l}\text { ACCACGACTAGCCGATACTAGCTACA- } \\
\text { TATATATTCCTA }\end{array}$ & Cloning & \\
\hline amiRNA440-200D & $\begin{array}{l}\text { TCCACGAGTAGCTGATACCAGCTCTC- } \\
\text { TTTTGTATTCC }\end{array}$ & Cloning & \\
\hline miRNA 194 & $\begin{array}{l}\text { AGAACACGGGGGACGAGCTCGGTAC- } \\
\text { CTAGGAATATATATGTAG }\end{array}$ & Cloning & Kpnl-BamHI \\
\hline miRNA 195 & $\begin{array}{l}\text { AAAGCTCTGCAGGTCGACTCTAGAGG- } \\
\text { ATCCGGAATACAAAAGAGAG }\end{array}$ & Cloning & Kpnl-BamHI \\
\hline KANA-F & GCCCCTGATGCTCTTCGTC & PCR & \\
\hline KANA-R & CTCTGATGCCGCCGTGTTCC & PCR & \\
\hline Actin8-F & GGTTTTCCCCAGTGTTGTTG & PCR & \\
\hline Actin8-R & CTCCATGTCATCCCAGTTGC & PCR & \\
\hline QPCR-At4g650-F & GTTTAACAAGTACAGCGAGG & q-PCR & \\
\hline QPCR-At4g650-R & GCAGTTCTCAACATGCGAC & q-PCR & \\
\hline QPCR-At4g660-F & CAGCCTTGGAAGTAGCAGG & q-PCR & \\
\hline QPCR-At4g660-R & CGAAAGGAAGCGATAAGCCA & q-PCR & \\
\hline QPCR-At5g430-F & CAACGGCGATTCAAACAACG & q-PCR & \\
\hline QPCR-At5g430-R & TGCTCCTCGGTCTGAAATG & q-PCR & \\
\hline QPCR-At5g440-F & ACGGCGGCGATTTCTTCTC & q-PCR & \\
\hline QPCR-At5g440-R & GATTTCGCTTACCTGGTCCA & q-PCR & \\
\hline QPCR-At2g490-F & AGCCCTTTTGCAGACTTCTC & q-PCR & \\
\hline QPCR-At2g490-R & GGTTTTAGCGTGCCTTCTTTG & q-PCR & \\
\hline QPCR-At2g500-F & GACCCGACGCATGCAAAG & q-PCR & \\
\hline QPCR-At2g500-R & ACGGCATCCCAAGCGTATG & q-PCR & \\
\hline QPCR-At1g460-F & GCTACCAGGAGCCAGTATC & q-PCR & \\
\hline QPCR-At1g460-R & GTGCCTGCATCAACTTTTTG & q-PCR & \\
\hline QPCR-At1g470-F & TTACGTGGCTGAAAGGTGTG & q-PCR & \\
\hline QPCR-At1g470-R & TTCCTCTGTTTTGTGCCCCT & q-PCR & \\
\hline QPCR-At1g840-F & GCTTCCTGGTGTGAAAAGAG & $q-P C R$ & \\
\hline QPCR-At1g840-R & TTCCCGCCTGTTGTAGTG & q-PCR & \\
\hline QPCR-At1g850-F & CTTTCGTGTTGCATTGCCTG & q-PCR & \\
\hline QPCR-At1g850-R & GTTTGCAAACTGTCTGCTCC & q-PCR & \\
\hline QPCR-At1g560-F & GCAAGACGACTGTTACAAGCTC & q-PCR & \\
\hline QPCR-At1g560-R & TTTCTCTTCCTCCGCCTTGTG & $q-P C R$ & \\
\hline QPCR-At4g200-F & TTATCAGCTACTCGTGGCATGG & q-PCR & \\
\hline QPCR-At4g200-R & TCCAAAGCCAGCTTCACATC & q-PCR & \\
\hline QPCR-PP2A_F & CCTGCGGTAATAACTGCATCT & q-PCR & \\
\hline QPCR-PP2A_R & CTTCACTTAGCTCCACCAAGCA & q-PCR & \\
\hline QPCR-RPL2-F & CGTGGTGTTGCTATGAATCC & q-PCR & \\
\hline QPCR-RPL2-R & GTCAGCTTTGGCAGCAGTAG & q-PCR & \\
\hline QPCR-SI23.8M-F & CGTGGCGTTGATGTTGAC & q-PCR & \\
\hline QPCR-SI23.8M-R & CAATTGGCTCACGCTCCT & q-PCR & \\
\hline Seq-At1g470-F & GAATCTTTGCATCGGCGTAT & Sequencing & \\
\hline Seq-At1g470-R & GGAGTCTGCAATGCAACTTG & Sequencing & \\
\hline Seq-At1g560-F & GTTGCTTGTTAAGTATGAACC & Sequencing & \\
\hline Seq-At1g560-R & GTTTCGATCCAAACTTCTAATG & Sequencing & \\
\hline pJET fwd & CGACTCACTATAGGGAGAGCGGC & Sequencing & \\
\hline pJET rev & AAGAACATCGATTTTCCATGGCAG & Sequencing & \\
\hline T7-F & TAATACGACTCACTATAGGG & Sequencing & \\
\hline
\end{tabular}




\begin{tabular}{lll}
\hline T7-R GCTAGTTATTGCTCAGCGG Sequencing & S
\end{tabular}

\subsubsection{Equipment}

The following table lists the equipment used for this study.

Table 3.17. Equipment

\begin{tabular}{|ll|}
\hline Equipment & Supplier \\
\hline Ab Sciex 6500 QTRAP® tandem mass & AB Sciex, Framingham (MA, USA) \\
spectrometer & \\
Arium pro-Ultrapure Water System & Sartorius AG (Goettingen, Germany) \\
Avanti J-25 centrifuge & Beckmann Coulter GmbH (Krefeld, Germany) \\
Centrifuge 5415 D & Eppendorf AG (Hamburg, Germany) \\
Centrifuge 5417 R & Eppendorf AG (Hamburg, Germany) \\
Centrifuge 5810 R & Eppendorf AG (Hamburg, Germany) \\
Climate chambers & YORK Refrigeration, YORK Industriekaelte \\
& GmbH \& Co. KG (Mannheim, Germany) \\
Epoch Microplate Spectrophotometer & BioTek (Winooski, U.S.A) \\
GC-2010 Plus equipment & Shimadzu (Kyoto, Japan) \\
JA10 rotor & Beckman Coulter GmbH (Krefeld, Germany) \\
JA25.50 rotor & Beckman Coulter GmbH (Krefeld, Germany) \\
Lyophilizer & Leybold-Heraeus GmbH (Cologne, Germany) \\
Mastercycler personal & Eppendorf AG (Hamburg, Germany) \\
Mixer Ball Mill MM200 with stainless steel & Retsch GmbH (Haan, Germany) \\
grinding jars or PTEE-jars & \\
Mini-PROTEAN3 Electrophoresis System & Bio-Rad, Hercules (CA, USA) \\
NanoDrop 2000 spectrophotometer & Thermo Fisher Scientific (Waltham, USA) \\
PCR detection systems iQ5 real-time & Bio-Rad Laboratories GmbH (Munich, Germany) \\
Percival CU-36L/D & Percival Scientific Inc. (Perry, USA) \\
Premium Freezer & Liebherr (Bulle, Switzerland) \\
Quartz SUPERSIL cuvettes & Hellma Analytics (Muellheim, Germany) \\
Sterile bench Prettl-Telstar Bioll-A & Telstar (Terrassa, Spain) \\
Microscope Leica MZ16F & Leica Microsystems (Wetzlar, Germany) \\
Olympus BH2 microscope & Olympus Corporation (Tokyo, Japan) \\
Conductometer & Twin Compact Meter-Horiba (Northampton, UK) \\
Electroporator & Bio-Rad “Gene Pulser" (CA, USA) \\
&
\end{tabular}

\subsubsection{Software packages}

All software and web-based services used in this study together with the correspondent reference are presented in Table 3.18.

Table 3.18. Software programs used in the course of this study

\begin{tabular}{|ll|}
\hline Software & Reference/Manufacturer \\
\hline Microsoft Office 2016 & Microsoft Corporation (Redmond, USA) \\
CorelDRAW & Corel Corporation \\
Snapgene Software & GSL Biotech \\
Infostat version 2008 & National University of Córdoba (Córdoba, \\
& Argentina) \\
Tomato analyzer 3.0 & [Rodriguez, 2010] \\
Geneious 8.1 & Biomatter Ltd. (Auckland, New Zealand) \\
Proteome Discoverer & Thermo Fisher Scientific (Waltham, USA) \\
ImageJ 1.4 & National Institutes of Health (Bethesda, USA)
\end{tabular}


GCMSsolution Software

National Center for Biotechnology Information

Analyst software 1.6.2

NanoDrop

iQ5 Optical System Software

SigmaPlot

Spectra Manager II Software

The Arabidopsis Information Resource (TAIR)

The Bio-analytic Resource for Plant Biology

database

The Universal Protein Resource (UniProt)

The PlantCare database

PLACE

Ensembl Plants database

The MEME Suite

Plant-DHS database

WMD - Web Micro RNA designer

Clustal Omega

CLC Sequence Viewer 7.0.2

Genomatix software suite v3.10

STRING

Panther
Shimadzu (Kyoto, Japan)

NCBI (Bethesda, USA)

Applied Biosystems (Darmstadt, Germany)

Thermo Fisher Scientific (Waltham, USA)

Bio-Rad Laboratories GmbH (Munich, Germany)

Systat Software GmbH (Erkrath, Germany)

JASCO Corporation (Hachioji, Japan)

http://www.arabidopsis.org/

[Winter, 2007]

The UniProt Consortium, 2018

[Lescot, 2002]

[Higo, 1999]

[Kersey, 2018]

[Bailey, 2009]

[Zhang, 2016]

http://wmd3.weigelworld.org/

[Sievers, 2018]

Qiagen (Venlo, Netherlands)

[Cartharius, 2005]

[Szklarczyk, 2015]

[Mi, 2016]

\subsection{Methods}

\subsubsection{Plant growth conditions}

For sterile culture, $A$. thaliana seeds were first exposed to $70 \%$ ethanol (v/v) for $15 \mathrm{~min}$ in a laminar flow hood. Immediately afterward, ethanol was discarded and $100 \%$ ethanol was added to the reaction tubes containing the seeds. After $60 \mathrm{~s}$, ethanol was removed and the open tubes were placed for several hours into the laminar flow hood to completely remove residual ethanol. The sterile and dry seeds were spread on $1 / 2 \mathrm{MS}$ plates containing $0.8 \%(\mathrm{w} / \mathrm{v})$ agar (Duchefa Biochemie B.V, Haarlem, The Netherlands). To synchronize germination, sealed plates with surgical tape (3M Deutschland $\mathrm{GmbH}$, Neuss, Germany) were incubated in the dark at $4{ }^{\circ} \mathrm{C}$ for $3 \mathrm{~d}$. The plates were then transferred to a climate chamber with long day conditions ( $16 \mathrm{~h} \mathrm{light} / 8 \mathrm{~h}$ dark, $22^{\circ} \mathrm{C}, 60 \%$ humidity, and light intensity of $100 \mu \mathrm{mol} \mathrm{m}^{-2} \mathrm{~s}^{-1}$ ) until seedlings were at the proper age for experiments or to be transferred to soil. The soil (Fruhstorfer Erde Typ T fein, Hawita Gruppe GmbH, Vechta, Germany) was steamed for $8 \mathrm{~h}$ at $80^{\circ} \mathrm{C}$ and chilled before being used. Seedlings were carefully placed in soil, covered with a plastic hood for 4 to 5 days and transferred to the climate chamber. For plants transformation, more inflorescence meristems were induced by cutting the first inflorescence. For experiments where sterile conditions were not needed, seeds were directly placed in soil, stratified for $3 \mathrm{~d}$ at $4^{\circ} \mathrm{C}$ in the dark and transferred to the climate chamber. 
Tomato seeds were sterilized with sodium hypochlorite $50 \%(\mathrm{v} / \mathrm{v})$ in a laminar flow hood. After $15 \mathrm{~min}$, the solution was removed and seeds were washed three times with sterile distilled water. Finally, water was discarded and seeds were placed on Petri plates or Magenta boxes containing 1/2 MS and Gamborg's B5 vitamins [Gamborg, 1968] with $0.9 \%$ (w/v) agar. Other seeds were placed in soil without sterilization. Tomato growth conditions were set to $25^{\circ} \mathrm{C}, 14$ $\mathrm{h}$ light/10 h dark and $70 \%$ humidity. All plants were maintained under optimal irrigation and controlled conditions.

\subsubsection{Plant treatments}

A. thaliana Col-0 and transgenic plants were investigated at different development stages, from seeds, seedlings of 7- to 10-day old and during the complete growth cycle until senescence was reached. Unless otherwise is specified, the conditions of $150 \mathrm{mM}$ sodium chloride $(\mathrm{NaCl})$, 300 mM Mannitol, $10 \mu \mathrm{M}$ Methyl Viologen (Sigma-Aldrich Co., St. Louis, USA), $4 \stackrel{\circ}{\circ}$ and $37 \stackrel{\circ}{\circ}$ were applied to seedlings for $3 \mathrm{~h}$. The response of genes, promoter activities, metabolites, and other measurements along with the phenotype of treated plants was investigated.

Tomato WT and transgenic plants were studied at the fruit level. For that purpose, fruit ripening was investigated on the vine (fruit was allowed to ripen on the plant) and on prechilled fruit (fruit was harvested at the green mature stage, stored for 28 days at $4{ }^{\circ} \mathrm{C}$, and then transferred to the climate chamber for ripening). Fruit was collected for experiments at the green mature (GM) stage and prechilled $(\mathrm{CH})$, which corresponds to prechilled fruit that was transferred for 1 day to the climate chamber. Fruit was all in comparable age and size, between 1 and $2 \mathrm{~g}$ weight and harvested $3 \mathrm{~h}$ after the light period began. Pericarp tissue of harvested and treated fruit was obtained by removing the locule tissues, skin, and seeds and was immediately processed or frozen in liquid nitrogen and stored at $-80^{\circ} \mathrm{C}$ until analysis.

A minimum of three biological replicates was analyzed in all $A$. thaliana and tomato experiments.

\subsubsection{Molecular biological methods}

Unless otherwise is specified, all methods were developed by following the protocols described in [Maniatis, 1989] and [Sambrook, 2001].

\subsubsection{Plasmids DNA extraction}

Bacterial clones were inoculated in $2 \mathrm{~mL}$ LB with the correspondent antibiotic and shaken overnight at $37^{\circ} \mathrm{C}$ in the case of $E$. coli or at $28^{\circ} \mathrm{C}$ for $A$. tumefaciens. On the next day, cells 
were centrifuged at $5,000 \times \mathrm{g}$ for $5 \mathrm{~min}$ and the pellet was used to isolate plasmids with the GenElute HP Plasmid Miniprep kit, following the manufacturer's instructions.

\subsubsection{Isolation of DNA from $\boldsymbol{A}$. thaliana and tomato plants}

For genomic DNA isolation, Arabidopsis and tomato leaves were placed in a $1.5 \mathrm{~mL}$ reaction tube and ground to a fine powder with a precooled pestle in liquid nitrogen. Two hundred and fifty $\mu \mathrm{L}$ of $\mathrm{CTAB}$ extraction buffer was added to the powder and incubated for $15 \mathrm{~min}$ at $65^{\circ} \mathrm{C}$. Two hundred and fifty $\mu \mathrm{L}$ of chloroform: isoamyl alcohol $(24: 1 \mathrm{v} / \mathrm{v})$ were added, the mixture was then briefly shaken and centrifugated for $15 \mathrm{~min}$ at 7,500 $\mathrm{xg}$ and room temperature. After phase separation, $200 \mu \mathrm{L}$ of the aqueous phase was transferred to a clean tube and $200 \mu \mathrm{L}$ of isopropanol was added. Tubes were inverted 8-10 times, incubated at room temperature for $20 \mathrm{~min}$ and centrifuged for $10 \mathrm{~min}$ at room temperature and 20,000 $\mathrm{xg}$ to sediment the DNA. The pellet was washed with $70 \%$ ethanol and dried by leaving the open tubes inverted on a paper towel for 5 to $10 \mathrm{~min}$. The DNA pellet was finally dissolved in $70 \mu \mathrm{L}$ of distilled water.

\subsubsection{Isolation of RNA from tissues plants}

For total RNA isolation from vegetative tissue, $100 \mathrm{mg}$ of plant material were collected, immersed in liquid nitrogen and pulverised to a fine powder with a precooled pestle. One $\mathrm{mL}$ of TriPure Isolation Reagent (Sigma-Aldrich Co., St. Louis, USA) was quickly added and the suspension was mixed roughly and incubated for $5 \mathrm{~min}$ at room temperature. Five hundred $\mathrm{mL}$ of cold chloroform were added to the tubes, the samples were then mixed and centrifuged at $12,0000 \mathrm{xg}$ and $4{ }^{\circ} \mathrm{C}$ for $15 \mathrm{~min}$. The clarified supernatant was put into a new tube, $600 \mu \mathrm{L}$ of isopropanol was added and the sample was mixed and incubated at room temperature for 30 min to precipitate the RNA. The, it was centrifuged for $10 \mathrm{~min}$ at $12,000 \times \mathrm{g}$ and $4{ }^{\circ} \mathrm{C}$ and the pellet was washed with cold $70 \%$ ethanol twice and air-dried before redissolving in $20 \mu \mathrm{L}$ RNase-free water. RNA concentrations were measured by using a NanoDrop 2000 spectrophotometer and the quality was confirmed by loading $1 \mu \mathrm{g}$ of sample in a $1.5 \%(\mathrm{w} / \mathrm{v})$ agarose gel. RNA samples were stored at $-80^{\circ} \mathrm{C}$.

\subsubsection{4. cDNA synthesis}

After RNA isolation, contaminant DNA was digested and removed from suspensions by using DNase I following the manufacturer's instructions: $2 \mu \mathrm{L}$ of $10 \mathrm{x}$ DNase buffer and 2 units of DNase I were incubated with 1 - $2 \mu \mathrm{g}$ of RNA for $30 \mathrm{~min}$ at $37^{\circ} \mathrm{C}$. To stop the reaction $2 \mu \mathrm{L} 50$ $\mathrm{mM}$ EDTA were added to the tube and incubated at $65^{\circ} \mathrm{C}$ for $10 \mathrm{~min}$. The reverse transcription of RNA into cDNA was performed by initially incubated the RNA with $1 \mu \mathrm{L} 0.5 \mu \mathrm{g} / \mu \mathrm{L}$ oligo dT18-primer at $65{ }^{\circ} \mathrm{C}$ for $10 \mathrm{~min}$. After this incubation, $4 \mu \mathrm{L}$ of $5 \mathrm{x}$ buffer for the reverse transcriptase, $2 \mu \mathrm{L}$ of $10 \mathrm{mM}$ dNTP mix, and $1 \mu \mathrm{L}$ (200 units) of RevertAid transcriptase were 
added to a final volume of $20 \mu \mathrm{L}$. The reaction mixture was transferred to a thermocycler at 37 ${ }^{\circ} \mathrm{C}$ for $1 \mathrm{~h}$ followed by $10 \mathrm{~min}$ at $70{ }^{\circ} \mathrm{C}$.

\subsubsection{Quantitative real-time PCR (q-PCR)}

After cDNA was synthesized, samples were checked for contamination by genomic DNA (gDNA) with RedTaq PCR (see sections 3.2.3.6.), using primers specific for actin (Actin8). These primers produce an amplicon of 1134 base pairs (bp) when cDNA is amplified, but 1409 bp is amplified if gDNA serves as a template. Quantitative real-time measurements of cDNA were performed by using the Takyon No Rox SYBR Core Kit blue dTTP following the manufacturers instructions and the iQ5 qPCR cycler. To design specific primers for the transcript of interest, the coding nucleotide sequence was first entered into the Primer3Prefold interface to find and exclude regions that can form secondary structures that may interfere in the amplification. Once these regions were excluded, the sequence was entered into the Primer3Plus interface [Untergasser, 2012] and specific oligonucleotides for q-PCR were selected. The software was set to get an amplicon length of 70-150 bp, primer length of 18-23 $\mathrm{bp}$ and melting temperatures of $58-62{ }^{\circ} \mathrm{C}$ with a difference of less than $3^{\circ} \mathrm{C}$ within the pair. For oligonucleotide sequences see section 3.1.10. The gene encoding ribosomal protein L2 (RPL2) was chosen as a reference gene in tomato measurements. The protein phosphatase $2 \mathrm{~A}$ gene (PP2A) was used as a reference in $A$. thaliana. Genes expression was normalized to these references genes to exclude changes due to any efficiency in the cDNA synthesis or alterations in the overall transcription because of the growth stage and external factors. Data analysis were performed by using the iQ5 software.

\subsubsection{Polymerase chain reaction (PCR)}

DNA sequences were amplified by PCR. Depending on the purpose, different DNA polymerases were used to amplify the sequences of interest. For cloning, Phusion Polymerase was used while to check the presence of specific DNA sequences, GoTaq and RedTaq were chosen. To identified and select positive bacterial clones that were transformed with a plasmid, colony PCR was performed by picking a small amount of bacterial cells from the agar plate and adding them to the PCR reaction mixture. Reactions were prepared following the manufacturer instructions and incubated in Mastercycler Personal thermocyclers to ensure the specific thermal conditions necessary for DNA denaturing, oligonucleotide annealing and elongation. Oligonucleotides used in the different PCR reactions are listed in section 3.1.10. 
Table 3.19. Thermocycler program for DNA amplification by PCR

\begin{tabular}{|ll|}
\hline Temperature & Time \\
\hline $98^{\circ} \mathrm{C}$ & $5 \mathrm{~min}$ \\
$94^{\circ} \mathrm{C}$ & $30 \mathrm{~s}$ \\
$55-60{ }^{\circ} \mathrm{C}$ & $30 \mathrm{~s}$ \\
$72{ }^{\circ} \mathrm{C}$ & $30 \mathrm{~s} / \mathrm{kb}$ \\
$72^{\circ} \mathrm{C}$ & $5 \mathrm{~min}$ \\
\hline \multicolumn{3}{|c|}{32 cycles } \\
\hline
\end{tabular}

\subsubsection{Agarose gel electrophoresis}

RNA samples, PCR products and restriction digests were separated in horizontal gels of 1 to $1.5 \%(\mathrm{w} / \mathrm{v})$ agarose. For this purpose, samples were mixed with 1 volume of $6 \times$ DNA loading dye. Since GoTaq and RedTaq reaction buffers contain a loading dye, no additional dye was added before loading the samples into the agarose gel. Gels were completely submerged in TAE buffer, samples were loaded and separated by applying a voltage of $10 \mathrm{~V} / \mathrm{cm}$ for $20 \mathrm{~min}$. Gels were then incubated in TAE buffer containing $2 \mu \mathrm{g} / \mathrm{mL}$ ethidium bromide for $10 \mathrm{~min}$ and DNA bands were finally visualized under UV light. Fragments were identified by comparing the size with 1 kilobase pair Gene-Ruler DNA ladder.

\subsubsection{Restriction, ligation and subcloning of DNA}

Double-stranded DNA was digested in reaction volumes of $20-50 \mu \mathrm{L}$ at $37^{\circ} \mathrm{C}$ for $1 \mathrm{~h}$ up to overnight. The procedure was according to the manufacturer's instructions. Digested fragments were then separated by electrophoresis in agarose gels and analyzed by UV light. For cloning, PCR products and digested fragments of the correct size were excised from agarose gels and extracted using the NucleoSpin Gel and PCR Clean-up kit. PCR products with blunt ends that were amplified by using Phusion polymerase were directly ligated into the pJET1.2/blunt and Zero blunt subcloning vectors following the manufacturer's instructions with some modifications. For ligations the final volume was $10 \mu \mathrm{L}$ and they were incubated for 30 min at room temperature. The fragment and the vector were used in a molar ratio of 3:1. After the incubation, the mixture was used to transform $100 \mu \mathrm{L}$ of chemically competent $E$. coli cells (for generation and transformation of competent cells see sections 3.2.3.11 and 3.2.3.12). Colony PCR was performed in several colonies to find positives bacterial clones that were successfully transformed. The positive clones were then used to inoculate $2 \mathrm{~mL} L B$ and plasmids were isolated as described in 3.2.3.1. To verify the correct insertion, plasmids were digested with the correspondent enzymes and the lengths of the resulting DNA fragments were compare to the expected size fragments. Verified plasmids were submitted to GATC (Biotech AG, Konstanz, Germany) or to the University of Maine DNA Sequencing Facility (Maine, USA) for sequencing. Sequencing results were aligned with the correct sequence to confirm the correctness of the insertion by using Geneious R8 software. 
Similarly, DNA fragments of interest were transferred from subcloning or entry vectors to other vectors such as binary vectors. In this case, the vector and the DNA fragment were digested with the same enzymes or with enzymes that generate compatible overhangs. The resulting DNA fragments were separated by gel electrophoresis in agarose and the bands were excised. Both the vector and the fragment to be inserted were purified, ligated and the ligation mixture was used for competent E. coli transformation as described in previous sections.

\subsubsection{Generation of microRNA silenced plants}

The generation of knock-down $A$. thaliana and tomato mutants were performed by using artificial microRNAs (amiR) according to [Schwab, 2006]. To design the amiR, the first step was to find conserved motifs in the transcripts of the genes to be silenced, by using The MEME suite. Adjacent regions to these motifs were also considered so as to obtain different sequences of 21 nucleotides length that could be used as amiR. All of these sequences were then deeply analyzed before choosing the best one that gave an amiR with the following requirements: 1 - no mismatches with the target sequences in positions 10 and 11, sites where argonaut produces the cleavage of the target strand, 2- no more than two mismatches with the target sequences in the first 5 nucleotides and 3 - it must start with $U$ at the 5 'end. Once the amiR for the genes of interest was found, the miRNA* was designed to match the amiR in the same way as in the duplex miR-miR ${ }^{*}$ of miRNA319. For $A$. thaliana mutants, the optimal amiR sequences were obtained from the WMD - Web Micro RNA designer tool. NB147 plasmid containing the MIR319a precursor was used to engineer the amiRs by replacing the original miR319a used as a template with the artificial sequence (21 nts), and the miR319a* with the sequence that pairs to the amiR with similar structural features as in the endogenous case. The amiR into the NB147 vector was sequenced and the correct fragment was subcloned into the binary vector pCHF3 for $A$. thaliana lines or MX202 for tomato. The binary vectors containing the amiRs were sequenced to confirm the insertion and used to transform $A$. tumefaciens cells by electroporation as described in section 3.2.3.13. Plants were transformed as stated in 3.2.4. A minimum of 3 independent transforming lines were isolated for each construct and used for further analysis.

\subsubsection{Construction of promoter-GUS fusion lines}

Promoter sequences were cloned in frame fused to the GUS reporter gene and GUS staining was performed as described in Arabidopsis: A Laboratory Manual [Weigel, 2002]. For this purpose, the $5^{\prime}$ genomic sequence upstream of the translation initiation codon of the query gene was amplified by PCR using specific primers (see section 3.1.10. for primers sequences). The promoter fragment was cloned into an entry vector as described in 3.2.3.8. The accuracy of the cloning was confirmed by sequencing and the fragment was then cloned in frame into 
the GF9 vector which contains the GUS gene.. The construct was once again sequenced to verify the correct insertion and used to transform $A$. tumefaciens cells by electroporation as described in section 3.2.3.13. A. thaliana plants were transformed by floral dip infiltration and positives transformant seeds were isolated.

\subsubsection{Generation of competent bacteria}

Competent bacteria were prepared for transformation by chemical treatment. For E. coli, 100 $\mu \mathrm{L}$ from overnight cultures of Top10 or DH5- $\alpha$ were used to inoculate $10 \mathrm{~mL}$ culture and shaked at $37^{\circ} \mathrm{C}$ until reaching an optical density at $600 \mathrm{~nm}(\mathrm{OD} 600)$ of $0.45-0.6$. The culture was then split into several $1.5 \mathrm{~mL}$ tubes and cells were sedimented at $5,000 \times \mathrm{g}$ and $4{ }^{\circ} \mathrm{C}$ for $5 \mathrm{~min}$. The supernatant was removed and the pellet was resuspended in $500 \mu \mathrm{L}$ of ice-cold $100 \mathrm{mM}$ calcium chloride $(\mathrm{CaCl})$. Cells were put on ice for $30 \mathrm{~min}$ and sedimented at 5,000 $\mathrm{xg}$ and 4 ${ }^{\circ} \mathrm{C}$ for $5 \mathrm{~min}$. Finally, cells were resuspended in $100 \mu \mathrm{L}$ of ice-cold $100 \mathrm{mM} \mathrm{CaCl}$ for subsequent transformation (see section 3.2.3.12.).

For the generation of A. tumefaciens competent cells, a GV2260 or GV3101 culture was used to inoculate a main culture that was shaken at $28^{\circ} \mathrm{C}$ overnight until getting an OD600 of 0.6. The cells were then placed in ice for $20 \mathrm{~min}$ and afterward sedimented at $7,000 \mathrm{xg}$ and $4{ }^{\circ} \mathrm{C}$ for $20 \mathrm{~min}$. The supernatant was thrown away and the cells were washed three times with icecold sterile water. After each wash, the cell suspension was centrifuged at $7,000 \mathrm{xg}$ for $10 \mathrm{~min}$ at $4{ }^{\circ} \mathrm{C}$, and the supernatant was removed. Cells were resuspended in sterile ice-cold $10 \%$ (v/v) glycerol and split into $1.5 \mathrm{~mL}$ tubes. Tubes were stored at $-80^{\circ} \mathrm{C}$.

\subsubsection{Transformation of $E$. coli}

E. coli competent cells were used for the amplification of DNA and plasmids and propagation of ligated DNA fragments. For transformations, $100 \mu \mathrm{L}$ of chemically competent cells were mixed with approximately $200 \mathrm{ng}$ of plasmid or with ligation mixtures. The cells and DNA were incubated $30 \mathrm{~min}$ on ice before being subject to a heat shock of $42^{\circ} \mathrm{C}$ for $45 \mathrm{~s}$. The cells and ligation mixture were then incubated on ice for $2 \mathrm{~min}$ and $900 \mu \mathrm{L}$ of LB media was added to the cells. The cells were shaken for $1 \mathrm{~h}$ at $37^{\circ} \mathrm{C}$ to recover. The cultures were then plated on LB agar plates with the appropriate antibiotic and incubated overnight at $37^{\circ} \mathrm{C}$.

\subsubsection{Transformation of Agrobacteria}

A. tumefaciens cells were transformed by electroporation. Two hundred $\mu \mathrm{L}$ competent cells were thawed on ice and mixed with $2 \mu \mathrm{g}$ of plasmid. The mixture was transferred to prechilled electroporation cuvettes with $0.1 \mathrm{~cm}$ gap distance before being placed in an electroporator. The electroporator was set up to use $25 \mu \mathrm{F}, 2,5 \mathrm{kV}, 400 \Omega$. After the pulse, the cuvettes were removed from the electroporator, $1 \mathrm{~mL}$ of $\mathrm{LB}$ media was added and the mixture was then 
transferred to a clean test tube. Cells were shaken at $28^{\circ} \mathrm{C}$ for 3 hours and then spread on LB agar plates with the correspondent antibiotics for clones selection. Plates were incubated for $2 \mathrm{~d}$ at $28{ }^{\circ} \mathrm{C}$. Positive individual colonies were confirmed by colony PCR and restriction digestion.

\subsubsection{Plant genetic transformation}

\subsubsection{Agrobacterium-mediated transformation of $\boldsymbol{A}$. thaliana}

Stable transformation of Arabidopsis plants was performed by flower dip with $A$. tumefaciens according to [Clough, 1998]. A $100 \mathrm{~mL}$ culture of transformed Agrobacteria in LB media with the correspondent antibiotics was shaken for $2 \mathrm{~d}$ at $28^{\circ} \mathrm{C}$ until reaching an OD600 of 0.6. Cells were then centrifuged at $7,000 \times \mathrm{g}$ and $4{ }^{\circ} \mathrm{C}$ for 20 min using an Avanti $\mathrm{J} 25$ centrifuge with a JA-10 rotor and resuspended in $100 \mathrm{~mL} 5 \%$ sucrose solution. $70 \mu \mathrm{L}$ Silwet L-77 silicone surfactant (Momentive Performance Materials Inc., Waterford, USA) was added just before dipping the flowers. The flowering inflorescences (grown for approximately 4 - 5 weeks in longday conditions, $16 \mathrm{~h}$ light/ $8 \mathrm{~h}$ dark, $22^{\circ} \mathrm{C}$ ) were dipped and gently agitated for several seconds into this bacteria suspension. After transformation, plants were placed horizontally and covered with a plastic hood overnight before being transferred to the climate chamber.

\subsubsection{Agrobacterium-mediated transformation of tomato}

Micro-Tom seeds were sterilized as described in section 3.2.1. and planted in seed germination medium (GM) (Table 3.4.). The seeds were transferred to the climate chamber for one week. Cotyledons of the 7-day-old seedlings were used for Agrobacterium transformation. Agrobacterium cultures were initiated from glycerol stocks or grown agar plates. Positive colonies were transferred to $3 \mathrm{~mL}$ LB containing the appropriate antibiotics and shaken at 28 ${ }^{\circ} \mathrm{C}$ for $48 \mathrm{~h}$. This saturated culture was then used to set a 1/1000 dilution in $100 \mathrm{~mL}$ LB which was shaken over night until an OD600 of 0.6 was reached. The bacterial cultures were centrifuged at $7,000 \times \mathrm{g}$ for 10 min using an Avanti J25 centrifuge with a JA-10 rotor and resuspended in $100 \mathrm{~mL}$ of $10 \mathrm{mM} \mathrm{MgSO} 4$ for the transformation of cotyledons. Cotyledons were divided into two halves across the midvein region and the edges were discarded. The resulting explants were completely dipped into the Agrobacterium suspension and gently shaken for $10 \mathrm{~min}$. The inoculated explants were transferred to a sterile filter paper to dry and then placed in co-cultivation medium (CM) (Table 3.5.) supplemented with $200 \mu \mathrm{M}$ acetosyringone (Sigma-Aldrich Co., St. Louis, USA) and incubated for $2 \mathrm{~d}$. After two days of co-culture, the cotyledons were transferred onto shoot induction medium (SIM). The explants were sub-cultured in fresh SIM medium every 2 weeks for shoot regeneration and elongation. 
When shoots were about 2-cm long, they were transferred to rooting media (RT) (Table 3.7.). The rooted plants were then transferred to soil and grown to maturity. One to three hundred explants were inoculated with every Agrobacterium construct to ensure a high number of transformant plants.

\subsubsection{Selection of transgenic plants}

After transformation, plants were grown as described in section 3.2.1. until senescence. Seeds were collected from Arabidopsis siliques and tomato fruit, surface sterilized and spread on $1 / 2$ MS plates containing kanamycin for selection of transformants. Leaves from transformant plants were used for DNA extraction and the presence of the transgene was verified by PCR. Seeds were collected from these kanamycin-resistant plants that were confirmed by PCR and were identified as the T1 generation. Seeds from the following generations were also selected using kanamycin and PCR, and the T2 generation was used for further experiments.

\subsubsection{Bioinformatics analysis}

All genes and proteins sequences were obtained from TAIR. The following online resources were used to investigate gene expressions and obtain additional information about the proteins of interest: BAR, Ensembl Plants database, TAIR, and UniProt. Particularly, the BAR database was used to study the response of genes to abiotic stress treatments including cold, heat, oxidative, osmotic, salt and drought. To study bidirectional and other promoters, sequences located upstream of the translation initiation codon of genes were downloaded from the TAIR database. These regions were analyzed by using the MEME interface to find conserved motif and the PLACE and PlantCare databases for regulatory elements. The bidirectional arrangement of the gene pairs was searched in other plants species to find conserved bidirectionality by using the ENSEMBL database.

\subsubsection{Protein manipulation}

\subsubsection{Protein isolation from $A$. thaliana plants}

Proteins were isolated from 15 day-old $A$. thaliana plants grown in $1 / 2$ MS by using a phenol extraction protocol [Grimplet, 2009] with some modifications. Approximately $0.5 \mathrm{~g}$ of seedlings were ground in liquid nitrogen to produce a fine powder. Four $\mathrm{mL}$ of extraction buffer $(0.7 \mathrm{M}$ sucrose, $0.5 \mathrm{M}$ Tris, $30 \mathrm{mM} \mathrm{HCl}, 50 \mathrm{mM}$ EDTA, $0.1 \mathrm{M} \mathrm{KCl}, 10 \mathrm{mM}$ Urea, $50 \mathrm{mM}$ DTT and 2 $\mathrm{mM}$ PMSF) was added to the plant material. Samples were incubated for $10 \mathrm{~min}$ at $4^{\circ} \mathrm{C}$ before centrifugation at $3,210 \times \mathrm{g}$ for $15 \mathrm{~min}$ at $4^{\circ} \mathrm{C}$. The supernatant was transferred to a new clean 
tube and $4 \mathrm{~mL}$ of extraction buffer saturated with phenol was added. Samples were then incubated on ice for $30 \mathrm{~min}$. Phases separation was achieved by centrifugation at 3,210 $\mathrm{g}$, $15 \mathrm{~min}$, and $4^{\circ} \mathrm{C}$. The top phase, containing phenol and proteins, was removed and transferred to a clean tube. An equal volume of extraction buffer was then added for re-extraction followed by another centrifugation at $3,210 \times \mathrm{g}, 15 \mathrm{~min}$, and $4^{\circ} \mathrm{C}$. The phenol phase containing the proteins was precipitated with 5 vol of $0.1 \mathrm{M}$ ammonium acetate in methanol, at $-20^{\circ} \mathrm{C}$ overnight. The precipitated was washed with $0.1 \mathrm{M}$ ammonium acetate in methanol and with cold acetone, before completely air dry the pellet. For resuspending the pellet $150 \mu \mathrm{L}$ of $6 \mathrm{M}$ Urea, $5 \%$ SDS was used.

\subsubsection{Gel-based proteomic analysis}

$60 \mu \mathrm{g}$ of each protein sample were loaded on a $10 \%$ SDS polyacrylamide gel and a subject to a current of 30 Milliamperes until samples had migrated $1 \mathrm{~cm}$ into the running gel. The gel was soaked into the staining solution of Coomassie Brilliant Blue G-250 [Neuhoff, 1988] and distained in water overnight. On the next day, gels were scanned and the bands were completely excised from the gels before being subjected to an in-gel tryptic digest as in [Shevchenko, 2007]. Briefly, gel pieces still blue were placed into Protein LoBind tubes and further distained with $50 \%(\mathrm{v} / \mathrm{v})$ acetonitrile containing $200 \mathrm{mM}$ ammonium bicarbonate for 30 $\min$ at $37^{\circ} \mathrm{C}$. Several times the destaining solution was changed until gel pieces were entirely colourless. To completely remove the rest of water, the gel was shaken for $10 \mathrm{~min}$ at room temperature in acetonitrile and then air dried for $15 \mathrm{~min}$. One hundred and fifty $\mu \mathrm{L}$ of $10 \mathrm{mM}$ dithiothreitol (DTT) was added to the gel pieces and incubated at $60^{\circ} \mathrm{C}$ for 15 min to reduce disulfide bonds. After removing the DTT, cysteine residues were alkylated by incubation for 45 min with $150 \mu \mathrm{l}$ of $55 \mathrm{mM}$ iodoacetamide at room temperature in the dark. Gel pieces were then washed twice with $150 \mu \mathrm{l} 100 \mathrm{mM}$ ammonium bicarbonate and $150 \mu \mathrm{l}$ of acetonitrile, and air dried for 10 min under the hood. Two hundred $\mu \mathrm{L}$ of trypsin buffer (5\% acetonitrile and 50 $\mathrm{mM}$ ammonium bicarbonate) containing trypsin ( $0.1 \mu \mathrm{g}$ to every $10 \mu \mathrm{g}$ of proteins) were added to the gel, incubated overnight at $37^{\circ} \mathrm{C}$, and then, centrifuged. The resulting supernatants were transferred to a new Protein LoBind tube. Peptides were extracted from the gel pieces with $100 \mu \mathrm{L}$ of $20 \mathrm{mM}$ ammonium bicarbonate and with $100 \mu \mathrm{l}$ of $5 \%(\mathrm{v} / \mathrm{v})$ formic acid in $50 \%$ acetonitrile at room temperature for $15 \mathrm{~min}$ each step. The extraction with acetonitrile/formic acid was repeated once and all supernatants of this and previews steps were combined in one tube. The supernatants were completely dry and the peptides were stored at $-20^{\circ} \mathrm{C}$ until measurements.

The peptides were analyzed with liquid chromatography-combined tandem mass spectrometry (LCMS) in the Service Unit LCMS Proteinanalytics at the University of Göttingen by the use of Orbitrap $^{\mathrm{TM}}$ mass spectrometers coupled to UltiMate3000 RSLCnano systems. Unless 
otherwise stated, all equipment and software used were obtained from Thermo Fisher Scientific, Waltham, USA. An Acclaim PepMap ${ }^{\text {TM }} 100$ pre-column was employed to trap and wash the peptides (C18 packing material, dimensions: $100 \mu \mathrm{m} \times 2 \mathrm{~cm}, 5 \mu \mathrm{m}$ particle size, 100 Å pore size) with $98 \%$ water, $2 \%$ acetonitrile, $0.07 \%$ trifluoroacetic acid with a flow rate of 25 $\mu \mathrm{L} / \mathrm{min}$ for $6 \mathrm{~min}$. Analytical separation of peptides was achieved by reverse phase chromatography using an Acclaim PepMap ${ }^{\mathrm{TM}}$ Rapid Separation Liquid Chromatography (RSLC) column (C18 packing material, dimensions: $75 \mu \mathrm{m} \times 15 \mathrm{~cm}, 2 \mu \mathrm{m}$ particle size, $100 \AA$ pore size) with a solvent gradient from $98 \%$ solvent A (99.9\% water, $0.1 \%$ formic acid) and $2 \%$ solvent B ( $80 \%$ acetonitrile, $19.9 \%$ water, $0.1 \%$ formic acid) to $40 \%$ solvent B within 40 min at a flow rate of $0.3 \mu \mathrm{L} / \mathrm{min}$. Online ionization was induced with a Nanospray Flex ${ }^{\mathrm{TM}}$ Ion Source at $2.4 \mathrm{kV}$. Mass over charge ratios of ionized peptides were determined using an Orbitrap ${ }^{\mathrm{TM}}$ Fourier Transformation Analyzer (mass over charge range: 300-1850, resolution: 30000) with parallel collision-induced dissociation fragmentation in a linear ion trap analyzer (Velos Pro ${ }^{\mathrm{TM}}$ ). The XCalibur 2.2 $2^{\mathrm{TM}}$ software was used for LCMS-method programming and data acquisition. Proteins were identified through peptide cross-correlation analyses against an $A$. thaliana database (Proteome ID UP000078284 obtained from UniProt) using the Proteome Discoverer $2.2^{\mathrm{TM}}$ software. The software was employed for data integration and analysis, and for the statistical quantitative comparisons between samples. Three biological replicates and two independent technical replicates were measured for control and mutant samples in each condition. Only proteins that showed significant fold change in respect to control samples were considered for further analysis. The overrepresented Gene Ontology in the list of proteins and the promising altered pathways were investigated by using STRING and Panther.

\subsubsection{Metabolite profiling by gas chromatography coupled to mass spectrometry (GC-MS)}

Metabolites were extracted from $100 \mathrm{mg}$ of ground leaf material. 15 day- and 28 day-old Arabidopsis plants were collected for this purpose. According to an established GC-MS protocol [Lisec, 2006], extractions were done by using a methanol/chloroform protocol followed by samples derivatization and measurement of the metabolite levels. Metabolites were identified by comparing to databases of authentic standards [Schauer, 2005] and quantified based on the internal standard added. Values were expressed as fold changes of metabolites in each sample relative to Col-0. 


\subsubsection{Analysis of the tomato lipidome by UPLC-nanoESI-MS/MS}

Tomato fruit pericarp was ground in liquid nitrogen to a fine powder with mortar and pestle and lyophilized before the extraction. Total lipid extraction was carried on as stated in [Grillitsch, 2014]. Briefly, $20 \mathrm{mg}$ of lyophilized powder were immersed in $6 \mathrm{~mL}$ of a hot propan-2ol/hexane/water $60: 26: 14(\mathrm{v} / \mathrm{v} / \mathrm{v})$ mixture at $60^{\circ} \mathrm{C}$ in $8 \mathrm{~mL}$ glass tube. The reaction mixture was incubated for $30 \mathrm{~min}$ at $60^{\circ} \mathrm{C}$ with shaking, samples were briefly vortexed and sonicated every 10 min during incubation. Extracts were then centrifuged at $20^{\circ} \mathrm{C}$ and $3,210 \times \mathrm{g}$ for $20 \mathrm{~min}$ and the clarified supernatant was collected in a new tube, dried under a stream of nitrogen and dissolved in $800 \mu$ tetrahydrofuran/methanol/water 4:4:1 (v/v/v). Samples were stored under argon at $-20^{\circ} \mathrm{C}$ until lipid measurements. In the case of PA and lyso-lipids analysis, $80 \mu \mathrm{L}$ of the sample were completely dried under streaming nitrogen and resolved in $400 \mu \mathrm{L}$ methanol. $6.5 \mu \mathrm{L}$ trimethylsilyl-diazomethane was added and the solution was shaken for $30 \mathrm{~min}$ at room temperature. $2 \mu \mathrm{L} 10 \%$ acetic acid was then added, the samples were mixed and dried under a stream of nitrogen. The dried pellet was finally dissolved in $80 \mu \mathrm{L}$ tetrahydrofuran/methanol/water $(4: 4: 1, \mathrm{v} / \mathrm{v} / \mathrm{v})$ and stored under argon at $-20{ }^{\circ} \mathrm{C}$ until being analyzed by LC-MS.

Analysis of lipids by UPLC-nanoESI-MS/MS was done by Dr. Cornelia Herrfurth (University of Göttingen). UPLC-nanoESI-MS/MS molecular species analysis was performed as previously described [Tarazona, 2015]. The peak area integration was done by using the Analyst Software peak-finding algorithm and a house-made software tool developed by Dr. Pablo Tarazona. The subsequent data analysis was carried on by calculating areas of lipid species within one lipid category relative to the total area in that group, and by comparing knockdown and WT results. Total peak areas of lipid categories and classes were also compared.

\subsubsection{Color and pigment content determinations}

For color evaluation of tomato, fruit was cleaned, dried with a clean paper towel and cut transversely through the center. Halves where immediately photographed by using a conventional scanner (Hewlett Packard) with the cut side down and a black background. The images were then analyzed by using the Tomato Analyzer 3.0 test. The software determines the colour parameters Red, Green, and Blue of the RGB color space. Besides this, the L*, $a^{*}$ and $b^{*}$ (numerical terms to express color from black to white, green to red, and blue to yellow axes, respectively) of the CIELAB color space and the Hue and Chroma color descriptors, were calculated. The scanner color calibration was achieved using Color Checker Munsell Color X-write.

For pigment extractions from $A$. thaliana leaves, $20 \mathrm{mg}$ of fresh material were used. Pigments were extracted two times with $80 \%(\mathrm{v} / \mathrm{v})$ and MES $10 \mathrm{mM} \mathrm{pH} \mathrm{5.9.} \mathrm{A} \mathrm{third} \mathrm{extraction} \mathrm{was} \mathrm{done}$ 
with $50 \%(\mathrm{v} / \mathrm{v})$ and MES $10 \mathrm{mM} \mathrm{pH} 5.9$ before all supernatants were combined and measured. Absorbances measured at 645,665 and $470 \mathrm{~nm}$ were used to calculate the ug of chlorophyll a, chlorophyll b and carotenoids per mg of fresh weigh [Lichtenthaler, 1986].

\subsubsection{Determination of proline content}

Proline was determined according to [Bates, 1973] with modifications. Twenty milligrams of $A$. thaliana leaves were extracted twice with $80 \%(\mathrm{v} / \mathrm{v})$ ethanol with $10 \mathrm{mM}$ MES pH 5.9 and once with $50 \%(\mathrm{v} / \mathrm{v})$ ethanol with $10 \mathrm{mM}$ MES pH 5.9. Each extraction step was performed for 30 $\min$ at $80^{\circ} \mathrm{C}$ and the supernatants were combined. For Pro determination, $100 \mu \mathrm{L}$ of the ethanol extract or Pro standard were mixed with $100 \mu \mathrm{L}$ ninhydrin reagent $[1 \%(\mathrm{w} / \mathrm{v})$ ninhydrin in $60 \%(\mathrm{v} / \mathrm{v})$ acetic acid] and heated at $95{ }^{\circ} \mathrm{C}$ for $20 \mathrm{~min}$. Absorbance measured at $520 \mathrm{~nm}$ was used to calculate $\mu \mathrm{mol}$ of Pro per gram of fresh weight tissue.

\subsubsection{Electrolyte leakage}

Fresh cubes of $5 \mathrm{~mm}^{2}$ from tomato pericarp and discs from $A$. thaliana leaves were thoroughly rinsed with deionized water, covered with $0.4 \mathrm{M}$ mannitol and incubated at $25{ }^{\circ} \mathrm{C}$ for $3 \mathrm{~h}$. A conductance meter (Twin Compact Meter-Horiba, Northampton, UK) was used to measure the conductivity of the at $25^{\circ} \mathrm{C}$ directly after the three hours of incubation and again after autoclaving samples for $30 \mathrm{~min}$ at $120{ }^{\circ} \mathrm{C}$ to release all the electrolytes. Electrolyte leakage was indicated as a percentage of total electrolytes. Measurements were carried out in several biological replicates.

\subsubsection{Enzyme activity analysis}

For enzyme activity measurements of catalase (CAT) and peroxidase (POX), $0.5-1 \mathrm{~g}$ of frozen tissues were ground in $0.6 \mathrm{~mL}$ of extraction buffer $\left(50 \mathrm{mM} \mathrm{K}_{2} \mathrm{PO}_{4} \mathrm{pH} 7.8\right.$ containing $20 \%$ glycerol and $2 \%$ polyvinylpolypyrrolidone) using mortar and pestle. Samples were transferred to a tube and centrifuged $10 \mathrm{~min}$ at $15,000 \times \mathrm{g}$. The clean supernatant was desalted by filtration through Sephadex G-25 that was previously equilibrated with the same extraction buffer. All the steps were performed at $4 \stackrel{\circ}{\circ}$. CAT activity was determined by the decrease in A240 of a mixture containing $10 \mathrm{mM} \mathrm{H}_{2} \mathrm{O}_{2}$ (extinction coefficient: $0.0394 \mathrm{mM}^{-1} \mathrm{~cm}^{-1}$ ), $50 \mathrm{mM}$ potassium phosphate $\mathrm{pH} 7.8$ and $1 \mathrm{mM}$ EDTA at $25^{\circ} \mathrm{C}$. POX activity was followed as the oxidation of guaiacol (extinction coefficient: $25.5 \mathrm{mM}^{-1} \mathrm{~cm}^{-1}$ ) at $470 \mathrm{~nm}$. The assay $(1 \mathrm{~mL}$ ) was carried out at $25^{\circ} \mathrm{C}$ and contained $0.017 \mathrm{M}$ guaiacol, $50 \mathrm{mM}$ potassium phosphate $\mathrm{pH}$ 7.8, $1 \mathrm{mM}$ EDTA. 
The reaction was initiated by the addition of $0.1 \mathrm{mM} \mathrm{H}_{2} \mathrm{O}_{2}$. Rates were corrected by chemical control experiments.

\subsubsection{Histochemical staining in A. thaliana tissues}

\subsubsection{GUS and Trypan blue staining}

GUS staining was performed according to the protocol described in Arabidopsis: A Laboratory Manual [Weigel, 2002]. To visualize GUS reporter activity under normal or stress conditions,

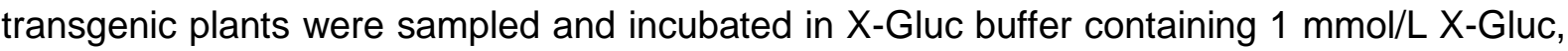
$100 \mathrm{mmol} / \mathrm{L}$ sodium phosphate buffer, $\mathrm{pH} 7.0,0.5 \mathrm{mmol} / \mathrm{L}$ potassium ferricyanide, $0.5 \mathrm{mmol} / \mathrm{L}$ potassium ferrocyanide and $0.1 \%(\mathrm{v} / \mathrm{v})$ Triton $\mathrm{X}-100$. The tissues were then infiltrated by vacuum in 3 cycles of $1 \mathrm{~min}$ and finally incubated overnight at $37^{\circ} \mathrm{C}$. Tissues were washed with $70 \%$ ethanol to remove pigments which may interfere with the proper display of dyed tissues. Pictures were taken by a binocular microscope Leica MZ16F. Trypan Blue staining was performed following a modified protocol [Fernández-Bautista, 2016]. Leaves from 28-days plants were incubated in trypan blue staining solution ( $85 \%$ lactic acid $10 \mathrm{~mL}$, phenol $10 \mathrm{~mL}$, $99 \%$ glycerol $10 \mathrm{~mL}$, distilled water $10 \mathrm{~mL}$ and trypan blue $40 \mathrm{mg}$ ). Time incubation was maximum $1 \mathrm{~h}$ before staining solution was completely removed. Seventy \% ethanol was added repeatedly until green tissues became completely colorless.

\subsubsection{NBT and DAB Staining}

$\mathrm{H}_{2} \mathrm{O}_{2}$ and superoxide anion were detected as described in [Scarpeci, 2008a]. For superoxide anion detection, leaves from 28 day-old plants were vacuum-infiltrated with $50 \mathrm{mM}$ sodium phosphate $\mathrm{pH} 7.5$ containing $0.2 \%$ (w/v) nitroblue tetrazolium (NBT; N6876, Sigma-Aldrich). Samples were incubated at room temperature for $2 \mathrm{~h}$ in the dark and then transferred to $70 \%$ ethanol. The reaction of the NBT with $\mathrm{O} 2 \cdot-$ produces a dark blue insoluble formazan compound. For the detection of $\mathrm{H}_{2} \mathrm{O}_{2}, 28$ day-old leaves were infiltrated with a solution of 1 $\mathrm{mg} / \mathrm{mL} 3,3^{\prime}$-diaminobenzidine (DAB). The DAB solution was freshly prepared prior incubation to avoid auto-oxidation. DAB was dissolved in $\mathrm{H}_{2} \mathrm{O}$ and adjusted to $\mathrm{pH} 3.8$ with $\mathrm{KOH}$. After incubation, leaves were cleared with $70 \%(\mathrm{v} / \mathrm{v})$ ethanol. $\mathrm{H}_{2} \mathrm{O}_{2}$ was visualized as a reddishbrown coloration due to DAB polymerization.

\subsubsection{Cell areas determination}

Epidermal cells areas were measured following a protocol given by Dr. Martin Mayta (IBRUNR-CONICET). To obtain views of epidermal cells, leaves from 21 day-old $A$. thaliana plants 
were incubated with lactic acid (85\%) at room temperature for 2 to 3 days, until tissues were completely cleared. Leaf cells were detected using differential interference contrast microscopy in an Olympus $\mathrm{BH} 2$ microscope. Photographs were taken along with a ruler that was used as a reference to calculate cells and total leaf area. To determine the mean areas, 6 leaves and a minimum of 650 cells from each mutant line or Col-0 plants were measured by using the ImageJ 1.4 software.

\subsubsection{Statistical Analyses}

Statistical significance was estimated according to Student's t-test using the Microsoft Excel software. Only comparisons with a $p$-value $<0.05$ were designated as statistically significant. One-way and two-way analysis of variance (ANOVA) followed by Fisher's multiple comparison test was carried on by using InfoStat. 


\section{Results and Discussion}

The results concerning the specific objectives of this study are presented and discussed separately in three independent chapters, which are found in the next sections:

\section{Chapter I:}

Putative bidirectional promoters regulate the expression of ACD genes in Arabidopsis thaliana under abiotic stress (section 4.1).

\section{Chapter II:}

Functional characterization of mitochondrial small heat shock proteins in Arabidopsis thaliana (section 4.2).

\section{Chapter III:}

Mitochondrial small heat shock protein and chilling tolerance in tomato fruit (section 4.3). 


\section{CHAPTER I}

\subsection{Putative bidirectional promoters regulate the expression of ACD genes in Arabidopsis thaliana under abiotic stress.}

Plants exhibit a high complexity in the sHSPs with members that are localized in the nuclearcytosolic compartment, chloroplasts, mitochondria, endoplasmic reticulum, and peroxisomes, indicating that these proteins are involved in the thermotolerance of practically all cellular compartments [Kotak, 2007]. Genes encoding sHSPs are highly expressed during heat stress, but in other stressing conditions including osmotic and oxidative stress, and UV-B exposure [Scarpeci, 2008b; Waters, 2013]. One of the main characteristics of the sHSPs is that they all share the conserved alpha-crystallin domain (ACD), a conserved carboxy-terminal domain of about 90 amino acids. However, not all the proteins that contain an ACD (ACD proteins) are sHSPs, some ACD proteins were described to have different functions. Plants have a large number of genes encoding sHSPs. To the date, 19 open reading frames (ORFs) encoding proteins related to the different classes of plants SHSPs have been identified in the Arabidopsis genome [Scharf, 2001]. It is not clear yet whether all SHSPs identified in Arabidopsis are all that exist, or if any of the ACD containing proteins identified are sHSPs [Bondino, 2012]. Therefore, attributing specific effects on individual ACD proteins is a great challenge. In a previews work, several genes encoding ACD proteins were identified in the Arabidopsis genome, many of them have not been studied yet raising the possibility that they could function as sHSPs. Notably, some of these genes are head-to-head oriented in the genome, sharing putative bidirectional promoters with other genes of different function (Bondino and Valle, unpublished result). Coupled transcription from bidirectional promoters is used by the cells as an additional mechanism for the regulation of gene expression. In plants, examples of bidirectional promoters responsive to stress conditions were reported, they involve genes of the chloroplast antioxidant defense [Bondino, 2009] or salt stress response [Banerjee, 2013]. Plant bidirectional promoters have gained considerable attention in recent years since they can be used for co-expressing multigene traits and facilitating crop improvement. In the present work, a functional characterization of head-to-head oriented genes encoding ACD proteins and the correspondent intergenic regions was performed. The results combined data obtained from bioinformatic predictions and published data, along with experimental evidence. 


\subsubsection{Characterization of four genes encoding ACD proteins with head-to-head orientation in the Arabidopsis thaliana genome.}

Among all genes encoding ACD proteins which are potential sHSPs, four genes were selected for this study considering the following criteria: the coding proteins should have an ACD domain in their sequences, they should share a putative bidirectional promoter (BDP) with another gene of different function and the intergenic region between them should be of no more than $1500 \mathrm{bp}$ long. The pair of genes selected in this way were: At4g25650-At4g25660, At5g51430At5g51440, At2g35490-At2g35500, At1g06460-At1g06470, where At4g25650, At5g51440, At2g35500 and At1g06460 encode for ACD proteins. The genes in each pair are located divergently and in opposite strands of the genome. As indicated by the gene identifier, the gene pairs are located in chromosomes 4, 5, 2 and 1, respectively. In the next paragraphs, a brief description of these genes is presented. Special attention was given to the responses of these genes to abiotic stresses.

Pair At4g25650-At4g25660: At4g25650 (2434 bp) encodes for an ACD protein (ACD1-LIKE, also called PTC52), component of a protochlorophyllide-dependent translocon. It is located in chloroplasts. This translocon is highly expressed in etiolated plants and may be involved in protein translocation processes and chlorophyll metabolism, although it has been reported that PTC52 is not strictly required for protein import in chloroplast [Bartsch, 2008; Boij, 2009]. At4g25660 (2190 bp) encodes for a putative thiol peptidase (PPPDE) present in the cytoplasm. The Bio-analytic Resource for Plant Biology database - BAR [Winter, 2007] was used to investigate the expression of the pair of genes in stress conditions, particularly in salt, osmotic, oxidative, high and low temperature. At4g25650 has a constitutive expression in the aerial parts of Arabidopsis plants. The expression increases after cold (12 h at $\left.4{ }^{\circ} \mathrm{C}\right)$, salt ( $3 \mathrm{~h} 150$ mM CINa), osmotic (3 h 300 mM Mannitol) and oxidative (3 h $10 \mu \mathrm{M}$ Methyl Viologen) stresses. No important changes were found after heat stress at $38^{\circ} \mathrm{C}$. At4g25660, on the other hand, is expressed in roots and leaves. The expression increases after cold (12 h at $4{ }^{\circ} \mathrm{C}$ ), and slightly under salt ( 6 h with $150 \mathrm{mM} \mathrm{CINa}$ ), osmotic (12 h $300 \mathrm{mM}$ Mannitol) and heat ( $6 \mathrm{~h}$ at $38^{\circ} \mathrm{C}$ ) conditions (see Suppl. Fig. 4.1).

Pair At5g51430-At5g51440: At5g51430 (4263 bp) encodes a protein homologous to Cog7 (EYE), a subunit of the oligomeric Golgi (COG) complex. EYE is probably involved in the transport or retention of proteins that are located in the Golgi complex and in the maintenance of Golgi function and morphology [Ishikawa, 2008]. At5g51440 (1254 bp) encodes a mitochondrial small heat shock protein (sHSP23.5). It has been established that only two mitochondrial sHSPs exist in A. thaliana, sHSP23.5 and sHSP23.6 [Waters, 2008a]. The 
information from the BAR database indicates that $A t 5 g 51430$ is expressed in roots and leaves of $A$. thaliana plants, it shows lower expression in cold ( $3 \mathrm{~h}$ at $4{ }^{\circ} \mathrm{C}$ ) and higher expression in salt $(6 \mathrm{~h} 150 \mathrm{mM} \mathrm{CINa})$ and heat $\left(1 \mathrm{~h}\right.$ at $\left.38^{\circ} \mathrm{C}\right)$ conditions. The other gene in the pair, At5g51440, has low basal expression in the whole plant but it is highly expressed under heat stress conditions ( $1 \mathrm{~h}$ at $38^{\circ} \mathrm{C}$ ) (see Suppl. Fig. 4.2 for this section).

Pair At2g35490-At2g35500: At2g35490 (1979 bp) encodes for a plastid-lipid associated protein, also named fibrillin2 (FIB2), which is located in chloroplasts. FIBs are thylakoidassociated proteins and accumulate under biotic and abiotic stress conditions [Youssef, 2010]. At2g35500 encodes the chloroplastic shikimate kinase-like 2 protein (SKL2) which has sequence similarity to shikimate kinases, albeit SKL2 may be inactivated with no SK activity [Fucile, 2008]. This gene has 2732 bp and the coding protein is also annotated as HSP20-like chaperone for the presence of the ACD domain. According to the BAR database, the pair At2g35490-At2g35500 is expressed in Arabidopsis plants in leaves but not in roots. At2g35490 shows high expression in normal conditions and it is not significantly affected by abiotic stresses. On the contrary, At2g35500 accumulates after cold ( $1 \mathrm{~h}$ at $\left.4{ }^{\circ} \mathrm{C}\right)$, oxidative $(6 \mathrm{~h} 10$ $\mu \mathrm{M}$ Methyl Viologen) and heat stress $\left(1 \mathrm{~h}\right.$ at $\left.38^{\circ} \mathrm{\circ}\right)$ treatments (see Suppl. Fig. 4.3).

Pair At1g06460-At1g06470: At1g06460 (2570 bp) encodes the protein ACD32.1, an alphacrystallin domain-containing protein with homology to sHSPs. It is one of the two sHSPs present in the peroxisome matrix where it may prevent the aggregation of partially denatured proteins in physiological and stress conditions [Ma, 2006]. The neighbor gene, At1g06470 has a sequence of $4869 \mathrm{bp}$ and encodes for a putative nucleotide/sugar transporter protein. The coding protein is located in the tonoplast. In Arabidopsis plants, At1g06460 exhibits high expression levels under salt ( 6 h 150 mM CINa), osmotic (6 h 300 mM Mannitol), oxidative (6 $\mathrm{h} 10 \mu \mathrm{M}$ Methyl Viologen) and heat stress ( $1 \mathrm{~h}$ at $\left.38^{\circ} \mathrm{C}\right)$ conditions, as can be found in the BAR database. At1g06470 is highly expressed in roots and leaves. The gene expression increases in salt (12 h $150 \mathrm{mM} \mathrm{CINa),} \mathrm{oxidative} \mathrm{(6} \mathrm{h} 10 \mu \mathrm{M}$ Methyl Viologen) and high temperature $\left(4 \mathrm{~h}\right.$ at $\left.38^{\circ} \mathrm{C}\right)$ conditions (Suppl. Fig. 4.4 ).

\subsubsection{Expression analysis of bi-directional genes under abiotic stress conditions.}

Based on the in-silico expression data, we designed experiments to study the expression of the selected genes in vivo. Quantitative analysis of the gene expressions in $A$. thaliana Col-0 plants was performed by q-PCR using cDNA, obtained from RNA isolated from 7 day-old seedlings. These plants were grown under normal conditions and exposed for $1 \mathrm{~h}$ to low 
temperature $\left(4^{\circ} \mathrm{C}\right)$, and $3 \mathrm{~h}$ to high temperature $\left(37^{\circ} \mathrm{C}\right)$, salinity $(150 \mathrm{mM} \mathrm{CINa})$, osmotic $(300$ $\mathrm{mM}$ Mannitol) and oxidative (10 $\mu \mathrm{M}$ Methyl Viologen, Sigma-Aldrich Co., St. Louis, USA) conditions. Samples from plants not exposed to stress were also taken and used as a control. The reference gene for the quantification analysis was protein phosphatase 2 ( $P P 2 a)$, a stressunrelated control gene. For each gene, the obtained data were normalized to the expression of the respective gene at control conditions. Expression of the genes sharing the same putative bidirectional promoter was always measured together to ensure the exact experimental conditions and comparable results.

Expression of At4g25650 and At4g25660: After $3 \mathrm{~h}$ under $\mathrm{NaCl}$ and Mannitol treatments, At4g25650 showed a 2.5-fold and 1.5-fold lower significant level in the expression, respectively (Fig. 4.1). Similarly, a significant reduction in the expression of this gene was found after the heat treatment (1.7-fold lower). In oxidative and cold conditions, no significant changes were observed, albeit a tendency to lower expression levels could be noticed (Fig. 4.1). While At4g25650 had lower expression levels in the abiotic stresses applied, At4g25660 presented the opposite behavior with an accumulation of transcripts under the same conditions, except for heat stress (Fig. 4.1). At4g25660 showed significant accumulation with 3.7-fold and 3-fold enrichment in salt and osmotic treatments, respectively. In the oxidative environment, the expression rates of At4g25660 also accumulated significantly with 2-fold higher expression, whereas no significant changes in the expression were obtained after heat and cold conditions (Fig. 4.1). Furthermore, the expression levels of the two genes were compared between each other. In this case, the raw quantitative data obtained of both genes was normalized to the basal expression of the $A t 4 g 25650$ gene in control conditions, and the relative gene expression of At4g25660 was calculated. In seedlings grown under normal conditions and not exposed to any external abiotic stress, At4g25660 already had a significantly higher (about 33-fold) expression compared to At4g25650. Under stress conditions, the same tendency was seen: At4g25660 transcripts highly accumulated compared to At4g25650 transcripts in all of them (data not shown). 


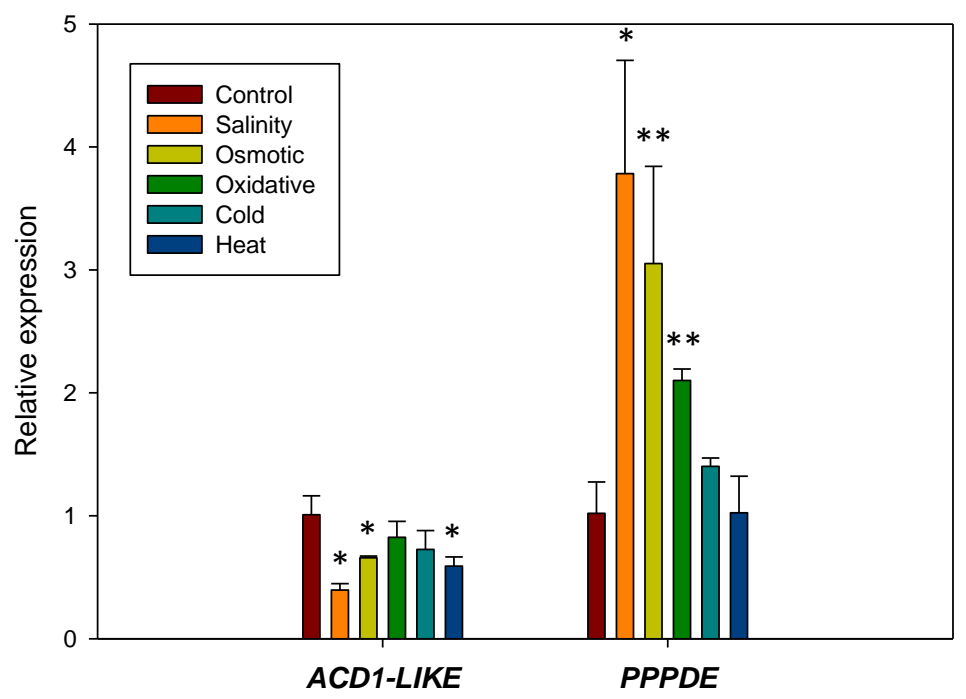

Figure 4.1. Expression of $A C D 1$-like and PPPDE in A. thaliana seedlings. Quantitative real-time PCR of $A C D 1$-like and PPPDE after salt, osmotic, oxidative, low and high temperature. Plants were grown for 7 days at $22{ }^{\circ} \mathrm{C}$ under long day conditions and exposed for $3 \mathrm{~h}$ to the different treatments. All expression values were first normalized to the PP2A expression taken as a reference. Relative expression of the transcripts was then normalized to their expression levels at control conditions. The experiment was performed twice, obtaining comparable results. Each point corresponds to the mean value + SD of three biological replicates of one experiment. Twenty plants were pooled for one replicate. Asterisks mean significance by one-sided t-test with ${ }^{*} \mathrm{p}<0.05,{ }^{* *} \mathrm{p}<.0 .01$.

Expression of $A t 5 g 51430$ and $A t 5 g 51440$ : The transcript levels of the genes in the pair At5g51430-At5g51440 displayed several changes in the tested conditions (Fig. 4.2). In the case of $A t 5 g 51430$, the expression rates significantly decreased under osmotic conditions (about 2-fold lower) while in the other stresses it showed similar expression levels to control plants. Two conditions affected the expression levels of At5g51440, salinity and high temperature. Under salt stress, a 2-fold reduction in the expression level was seen. The highest amount of transcripts for $A t 5 g 51440$ was observed upon heat stress, it accumulated to more than 400 times after $3 \mathrm{~h}$ at $37^{\circ} \mathrm{C}$. In another analysis, the raw quantitative data obtained of both genes was normalized to the basal expression of the At5g51430 gene in control conditions (now used as reference), and the relative gene expression of At5g51440 was calculated. The relative expression level between the two genes of the pair showed a considerable high expression for At5g51430 compared to At5g51440, it had 7-fold higher levels of transcripts already under normal conditions of growth. The only exception was at high temperature where $A t 5 g 51440$ was greatly expressed and accumulated at higher rates compared to its neighbor (data not shown). 


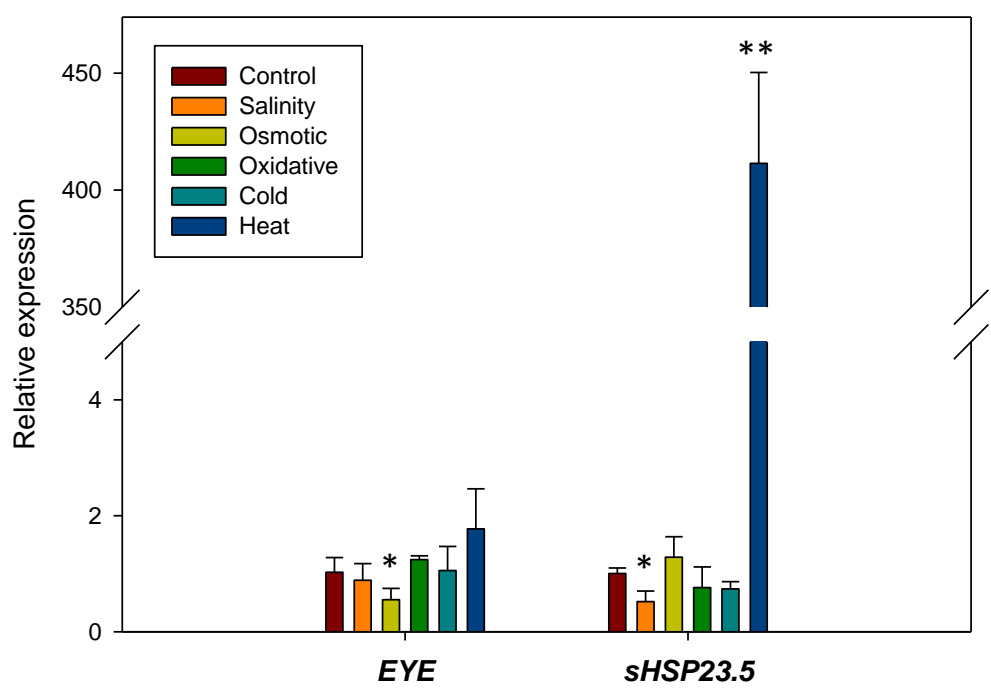

Figure 4.2. Expression of EYE and sHSP23.5 in A. thaliana seedlings. Quantitative real-time PCR of $E Y E$ and $S H S P 23.5$ after salt, osmotic, oxidative, low and high temperature. Plants were grown for 7 days at $22{ }^{\circ} \mathrm{C}$ under long day conditions and exposed for $3 \mathrm{~h}$ to the different treatments. All expression values were first normalized to the PP2A expression taken as a reference. Relative expression of the transcripts was then normalized to their expression levels at control conditions. The experiment was performed twice, obtaining comparable results. Each point corresponds to the mean value + SD of three biological replicates of one experiment. Twenty plants were pooled for one replicate. Asterisks mean significance by one-sided t-test with ${ }^{*} p<0.05,{ }^{* *} p<.0 .01$.

Expression of At2g35490 and At2g35500: The quantification of the expression levels of At2g35490 and At2g35500 in seedlings after the abiotic stresses showed similar expression patterns for both genes (Fig. 4.3). The transcripts of At2g35490 and At2g35500 significantly accumulated under low and high temperature: the expression levels were 1.4- to 1.8-fold higher at $4{ }^{\circ} \mathrm{C}$ and 1.3 - to 1.8 -fold higher at $37^{\circ} \mathrm{C}$, respectively. Beside this, At $2 \mathrm{~g} 35500$ had a slight, but significant, decrease of expression under oxidative stress. The same tendency was observed for At2g35490 but only when considering a statistical $p$-value of 0.1 . For the rest of the conditions that were analyzed no significant changes were noticed. In addition, the expression levels of the two genes were compared between each other. To do this, the raw quantitative data obtained of both genes measurements were normalized to the basal expression of the At2g35490 gene in control conditions, and the relative gene expression of At2g35500 was calculated. By performing this analysis, it was evident the higher relative expression of At2g35490 under control conditions and after the stress treatments (with fold changes from 4.4 in control and $37^{\circ} \mathrm{C}$ to 5.8 in osmotic stress) (data not shown). 


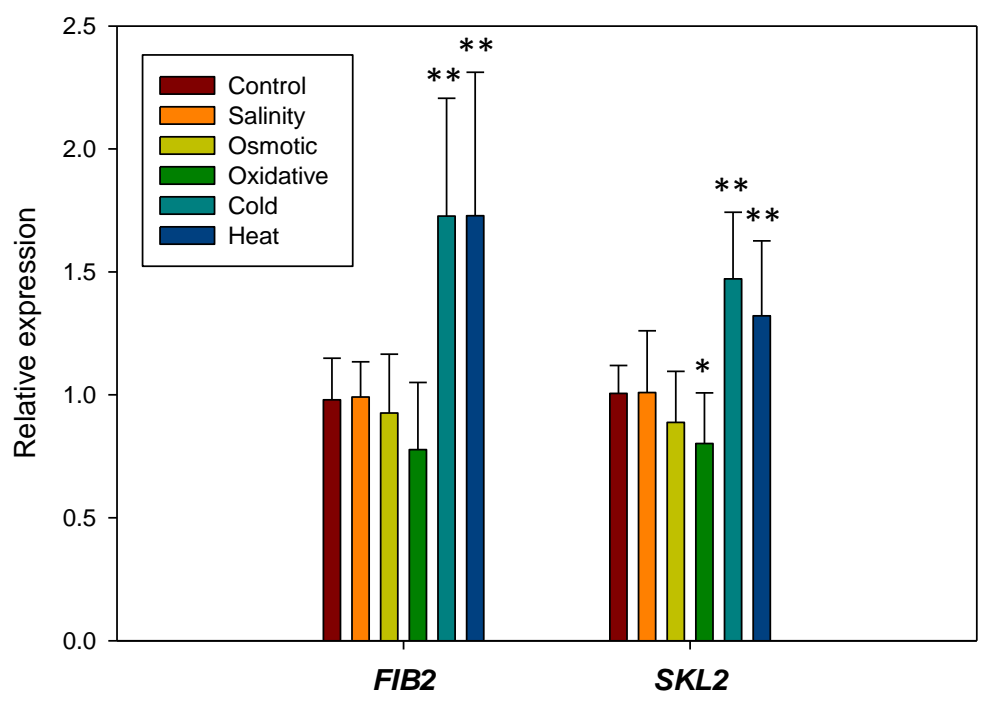

Figure 4.3. Expression of $F I B 2$ and $S K L 2$ in $A$. thaliana seedlings. Quantitative real-time PCR of FIB2 and SKL2 after salt, osmotic, oxidative, low and high temperature. Plants were grown for 7 days at $22{ }^{\circ} \mathrm{C}$ under long day conditions and exposed for $3 \mathrm{~h}$ to the different treatments. All expression values were first normalized to the PP2A expression taken as a reference. Relative expression of the transcripts was then normalized to their expression levels at control conditions. The experiment was performed twice, obtaining comparable results. Each point corresponds to the mean value + SD of three biological replicates of one experiment. Twenty plants were pooled for one replicate. Asterisks mean significance by one-sided t-test with ${ }^{*} p<0.05,{ }^{* *} p<.0 .01$.

Expression of At1g06460-At1g06470: The gene pair At1g06460-At1g06470 also displayed stress-induced responses in some of the tested conditions (Fig. 4.4). Under salinity, both genes exhibited a significant reduction of the expression. Under osmotic conditions, the expression levels of At1g06470 were reduced, but At1g06460 expression showed no significant change. Under high temperature condition, the expression response of seedlings was the opposite for these genes. That is, the expression of At1g06460 was reduced after the heat treatment while At1g06470 had a 1.5-fold enrichment. In the other stress conditions, both genes showed expression levels that were similar to the control conditions. Moreover, a comparison between the expression of both genes was performed by calculating the expression of At1g06470 relative to At1g06460. For this purpose, the raw quantitative data obtained of both genes measurements were normalized to the basal expression of the At1g06460 gene in control conditions. Under normal conditions of growth, At1g06460 displayed significantly higher expression in comparison to the At1g06470 expression, it accumulated to about 9-fold higher. This difference in the expression of the genes under control conditions was also found in the abiotic stress conditions (data not shown). 


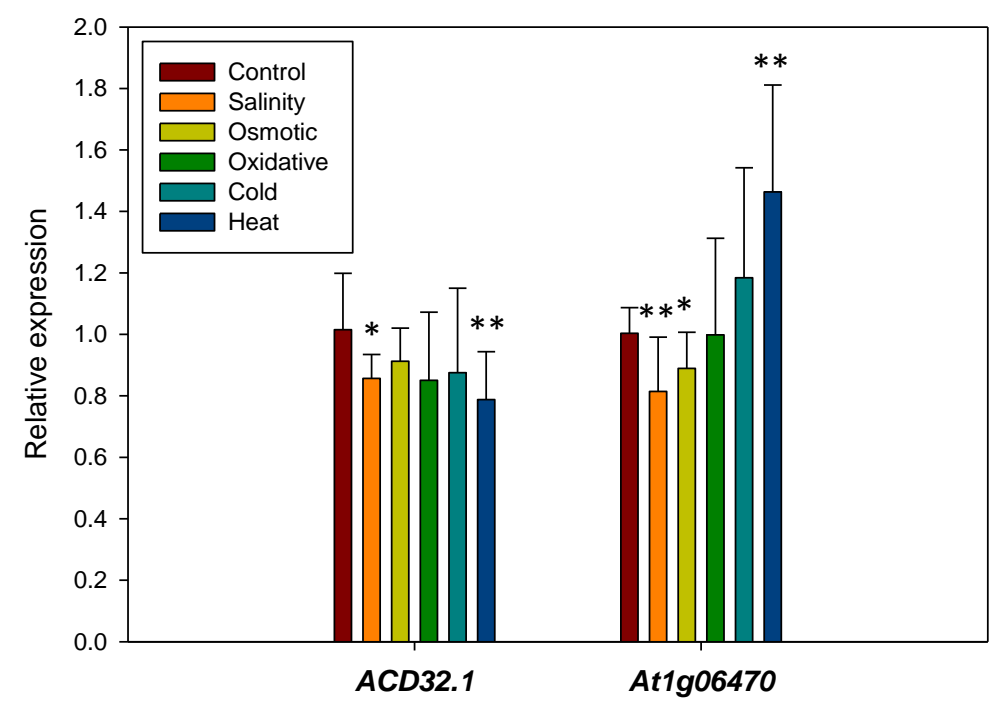

Figure 4.4. Expression of $A C D 32.1$ and $A t 1 g 06470$ in A. thaliana seedlings. Quantitative real-time PCR of ACD32.1 and At1g06470 after salt, osmotic, oxidative, low and high temperature. Plants were grown for 7 days at $22{ }^{\circ} \mathrm{C}$ under long day conditions and exposed for $3 \mathrm{~h}$ to the different treatments. All expression values were first normalized to the PP2A expression taken as a reference. Relative expression of the transcripts was then normalized to their expression levels at control conditions. The experiment was performed twice, obtaining comparable results. Each point corresponds to the mean value + SD of three biological replicates of one experiment. Twenty plants were pooled for one replicate. Asterisks mean significance by one-sided t-test with ${ }^{*} \mathrm{p}<0.05,{ }^{* *} \mathrm{p}<.0 .01$.

\subsubsection{Analysis of four putative bidirectional promoters in A. thaliana.}

The intergenic regions between the genes At4g25650-At4g25660, At5g51430-At5g51440, At2g35490-At2g35500, and At1g06460-At1g06470 were examined to identify regulatory motifs and transcription factor sites by using the PlantCare and PLACE databases. The PlantCare database is a useful resource for the in silico analysis of promoter sequences [Lescot, 2002]. Similarly, the PLACE database allows the identification of cis-acting regulatory DNA element in promoters [Higo, 1999].

\section{$\underline{\text { Intergenic region between } A t 4 g 25650 \text { and } A t 4 g 25660}$}

The region located between these genes is $523 \mathrm{bp}$ long. The analysis of the promoter sequence by using the PlantCare database has revealed the presence of different putative cis elements such as CAAT and TATA-BOXES, ABRE, Box II, G-Box, GA-motif, GATA-motif, GT1-motif, I-box and MYB recognition sites (Table 4.1). The CAAT and TATA boxes are common cis-acting elements that are essential for the transcription initiation and were identified in the positive and negative strand of the sequence. Several light responsive cis-elements were found in both directions of the sequence including the motifs Box II, G-Box, GA-motif, GATAmotif, GT1-motif, and I-box. The water stress related site MYB is present in the negative strand 
while two abscisic acid responsive elements, ABRE and ABRE2 are putatively located in the positive strand of the intergenic region. The promoter sequence also revealed several potential TFBs (Table 4.2). Among them, 18 water stress, 3 low temperature, and 7 light regulated TFBs were recognized. Interestingly, the promoter contains 14 TFBs related to plant hormones such as auxin, gibberellin, and cytokinin (SURECOREATSULTR11, ARR1AT, and WRKY71OS). Two TFBs present in the sequence were related to heat stress regulation (CCAATBOX1) and salinity stress (GT1GMSCAM4), both conditions affecting the expression of At4g25650 and At4g25660 (Fig. 4.1).

Table 4.1. List of putative cis-acting regulatory elements present in the putative BDP of At4g25650-At4g25660 (from Plant CARE).

\begin{tabular}{|c|c|c|c|c|c|}
\hline Site & Organism & Position & Strand & Sequence & Function \\
\hline \multirow[t]{2}{*}{ ABRE } & A. thaliana & 242 & + & ACGTG & \multirow{2}{*}{$\begin{array}{l}\text { Abscisic acid } \\
\text { response }\end{array}$} \\
\hline & A. thaliana & 241 & + & CACGTG & \\
\hline ABRE2 & Zea mays & 240 & + & CCACGTGG & $\begin{array}{l}\text { Abscisic acid } \\
\text { response }\end{array}$ \\
\hline Box II & $\begin{array}{l}\text { Petroselinum } \\
\text { crispum }\end{array}$ & 240 & + & CCACGTGGC & Light response \\
\hline \multirow[t]{3}{*}{ CAAT-box } & $\begin{array}{l}\text { Nicotiana } \\
\text { glutinosa }\end{array}$ & 425 & - & CAAT & \multirow{3}{*}{$\begin{array}{l}\text { Common cis- } \\
\text { acting element }\end{array}$} \\
\hline & $\begin{array}{l}\text { Nicotiana } \\
\text { glutinosa }\end{array}$ & 197 & + & CAAT & \\
\hline & Pisum sativum & 24 & + & CAAAT & \\
\hline CCAAT-box & Hordeum vulgare & 353 & + & CAACGG & $\begin{array}{l}\text { MYBHv1 binding } \\
\text { site }\end{array}$ \\
\hline \multirow{5}{*}{ G-Box } & Triticum aestivum & 239 & + & TCCACATGGCA & \multirow{5}{*}{ Light response } \\
\hline & Pisum sativum & 241 & + & CACGTG & \\
\hline & A. thaliana & 241 & + & CACGTG & \\
\hline & A. thaliana & 237 & + & CTTCCACGTGGCA & \\
\hline & A. thaliana & 239 & - & GCCACGTGGA & \\
\hline GA-motif & A. thaliana & 208 & + & ATAGATAA & Light response \\
\hline GATA-motif & A. thaliana & 215 & + & AAGATAAGATT & Light response \\
\hline \multirow[t]{2}{*}{ GT1-motif } & A. thaliana & 323 & - & GGTTAA & \multirow{2}{*}{ Light response } \\
\hline & Avena sativa & 322 & - & GGTTAAT & \\
\hline I-box & A. thaliana & 270 & + & GATAAGGGT & Light response \\
\hline $\begin{array}{l}\text { MYB } \\
\text { recognition } \\
\text { site }\end{array}$ & A. thaliana & 353 & - & CCGTTG & $\begin{array}{l}\text { Water stress } \\
\text { response }\end{array}$ \\
\hline \multirow[t]{8}{*}{ TATA-box } & A. thaliana & 65 & - & TATATAA & \multirow{8}{*}{$\begin{array}{l}\text { Core promoter } \\
\text { element }\end{array}$} \\
\hline & Brassica napus & 437 & + & ATTATA & \\
\hline & A. thaliana & 68 & + & TATA & \\
\hline & A. thaliana & 441 & - & TATATA & \\
\hline & A. thaliana & 66 & + & TATATA & \\
\hline & A. thaliana & 439 & - & TATATA & \\
\hline & Brassica oleracea & 123 & + & ATATAA & \\
\hline & A. thaliana & 443 & - & TATA & \\
\hline
\end{tabular}




\begin{tabular}{|lccll|}
\hline Brassica napus & 64 & + & ATTATA \\
A. thaliana & 438 & - & TATATAA \\
A. thaliana & 110 & + & TATA \\
Brassica napus & 440 & - & ATATAT \\
Brassica napus & 67 & + & ATATAT \\
A. thaliana & 124 & + & TATA \\
\hline
\end{tabular}

Table 4.2. List of putative transcriptional factor binding sites (TFBs) present in the BDP of At4g25650-At4g25660 (from PLACE database).

\begin{tabular}{|llclll|}
\hline \multicolumn{1}{|c}{ Place ID } & \multicolumn{1}{c}{ Function } & $\begin{array}{c}\text { Copy } \\
\text { number }\end{array}$ & Strand & Sequence & $\begin{array}{c}\text { Place } \\
\text { Accession }\end{array}$ \\
\hline SURECOREATSULTR11 & Auxin response & 1 & $(+)$ & GAGAC & S000499 \\
EVENINGAT & Circadian control & 1 & $(+)$ & AAAATATCT & S000385 \\
CAATBOX1 & Common cis-acting element & 7 & $(-)(+)$ & CAAT & S000028 \\
TATABOX2 & Common cis-acting element & 1 & $(-)$ & TATAAAT & S000109 \\
ARR1AT & Cytokinin response & 12 & $(-)(+)$ & NGATT & S000454 \\
DOFCOREZM & Endosperm specific & 11 & $(-)(+)$ & AAAG & S000265 \\
WRKY71OS & Gibberellin specific & 1 & $(+)$ & TGAC & S000447 \\
CCAATBOX1 & Heat shock response & 2 & $(+)$ & CCAAT & S000030 \\
GT1CONSENSUS & Light-regulated & 5 & $(-)(+)$ & GRWAAW & S000198 \\
IBOXCORE & Light-regulated & 2 & $(+)$ & GATAA & S000199 \\
LTREATLTI78 & Low temperature response & 1 & $(-)$ & ACCGACA & S000157 \\
LTRECOREATCOR15 & Low temperature response & 2 & $(-)$ & CCGAC & S000153 \\
CACTFTPPCA1 & Mesophyll Specific & 8 & $(-)(+)$ & YACT & S000449 \\
GT1GMSCAM4 & Salt-induced element & 1 & $(-)$ & GAAAAA & S000453 \\
ACGTATERD1 & Water stress response & 2 & $(-)(+)$ & ACGT & S000415 \\
CBFHV & Water stress response & 2 & $(-)$ & RYCGAC & S0000497 \\
DRECRTCOREAT & Water stress response & 2 & $(-)$ & RCCGAC & S0000418 \\
MYB1AT & Water stress response & 3 & $(-)(+)$ & WAACCA & S0000408 \\
MYB2CONSENSUSAT & Water stress response & 1 & $(+)$ & YAACKG & S000409 \\
MYBCORE & Water stress response & 4 & $(-)(+)$ & CNGTTR & S000176 \\
MYCCONSENSUSAT & Water stress response & 4 & $(-)(+)$ & CANNTG & S000407 \\
\hline
\end{tabular}

Intergenic region between $A t 5 g 51430$ and $A t 5 g 51440$

A 446 bp sequence is located between At5g51430 and At5g51440. The region contains the CAAT and TATA boxes and other cis-regulatory elements as was predicted by the PlantCare database (Table 4.3). The Box 4 at site -405 (ATTAAT), the HSE at site +378 (AAAAAATTTC), the TC-rich repeats at site +342 (ATTCTCTAAC) and GAG-motif at sites $-295,-322$ (AGAGAGT) are stress related cis-elements. Two methyl jasmonate sites were predicted in this sequence, CGTCA-motif, and TGACG-motif. In addition to this, water stress (CBFHV, DRECRTCOREAT, MYB1AT, MYBCORE, and MYCCONSENSUSAT) and light stress (GATABOX, GT1CONSENSUS, IBOXCORE, SORLIP2AT, TBOXATGAPB) related TFBs 
were predicted in the sequence (Table 4.4). Other putative TFBs that are associated with high temperature (PRECONSCRHSP70A), salinity (GT1GMSCAM4) and low temperature (LTRECOREATCOR15) were also identified in several sites along the sequence.

Table 4.3. List of putative cis-acting regulatory elements present in the putative BDP of At5g51430-At5g51440 (from Plant CARE).

\begin{tabular}{|c|c|c|c|c|c|}
\hline Site & Organism & Position & Strand & Sequence & Function \\
\hline \multirow{3}{*}{ ARE } & Zea mays & 107 & + & AAACCA & \multirow{3}{*}{$\begin{array}{l}\text { Cis-acting regulatory } \\
\text { element essential for } \\
\text { the anaerobic } \\
\text { induction }\end{array}$} \\
\hline & Zea mays & 152 & + & AAACCA & \\
\hline & Zea mays & 147 & + & AAACCA & \\
\hline Box 4 & $\begin{array}{l}\text { Petroselinum } \\
\text { crispum }\end{array}$ & 405 & - & ATTAAT & Light response \\
\hline \multirow[t]{3}{*}{ CAAT-box } & Pisum sativum & 111 & + & CAAAT & \multirow{3}{*}{$\begin{array}{l}\text { Common cis-acting } \\
\text { element }\end{array}$} \\
\hline & Pisum sativum & 180 & + & CAAAT & \\
\hline & Pisum sativum & 133 & + & CAAAT & \\
\hline CGTCA-motif & $\begin{array}{l}\text { Hordeum } \\
\text { vulgare }\end{array}$ & 206 & + & CGTCA & MeJA response \\
\hline HSE & $\begin{array}{l}\text { Brassica } \\
\text { oleracea }\end{array}$ & 378 & + & AAAAAATTTC & Heat stress response \\
\hline \multirow{4}{*}{ TATA-box } & A. thaliana & 332 & - & TATATA & \multirow{4}{*}{$\begin{array}{l}\text { Core promoter } \\
\text { element }\end{array}$} \\
\hline & A. thaliana & 334 & - & TATA & \\
\hline & $\begin{array}{l}\text { Brassica } \\
\text { oleracea }\end{array}$ & 333 & + & ATATAA & \\
\hline & A. thaliana & 374 & - & TATA & \\
\hline TC-rich repeats & $\begin{array}{l}\text { Nicotiana } \\
\text { tabacum }\end{array}$ & 342 & + & ATTCTCTAAC & Stress response \\
\hline TGACG-motif & $\begin{array}{l}\text { Hordeum } \\
\text { vulgare }\end{array}$ & 206 & - & TGACG & MeJA response \\
\hline \multirow[t]{2}{*}{ GAG-motif } & A. thaliana & 295 & - & AGAGAGT & \multirow{2}{*}{ Light response } \\
\hline & A. thaliana & 322 & - & AGAGAGT & \\
\hline
\end{tabular}

Table 4.4. List of putative TFBs present in the BDP of At5g51430-At5g51440 (from PLACE database).

\begin{tabular}{|c|c|c|c|c|c|}
\hline Place ID & Function & $\begin{array}{c}\text { Copy } \\
\text { number }\end{array}$ & Strand & Sequence & $\begin{array}{c}\text { Place } \\
\text { Accession }\end{array}$ \\
\hline SURECOREATSULTR11 & Auxin response & 1 & $(+)$ & GAGAC & S000499 \\
\hline EVENINGAT & $\begin{array}{l}\text { Circadian control } \\
\text { associated element }\end{array}$ & 1 & $(+)$ & AAAATATCT & S000385 \\
\hline TATABOX4 & $\begin{array}{l}\text { Common cis-acting } \\
\text { element }\end{array}$ & 1 & $(+)$ & TATATAA & S000111 \\
\hline TATABOX5 & $\begin{array}{l}\text { Common cis-acting } \\
\text { element }\end{array}$ & 3 & $(-)(+)$ & TTATTT & S000203 \\
\hline ARR1AT & Cytokinin response & 2 & $(-)(+)$ & NGATT & S000454 \\
\hline DOFCOREZM & Endosperm specific & 13 & $(-)(+)$ & AAAG & S000265 \\
\hline WRKY71OS & Gibberellin specific & 1 & $(-)$ & TGAC & S000447 \\
\hline PRECONSCRHSP70A & Heat response & 8 & $(+)$ & SCGAYNRNNNHD & S000506 \\
\hline GATABOX & Light-regulated & 3 & $(-)(+)$ & GATA & S000039 \\
\hline
\end{tabular}




\begin{tabular}{|llccll|} 
GT1CONSENSUS & Light-regulated & 8 & $(-)(+)$ & GRWAAW & S000198 \\
IBOXCORE & Light-regulated & 1 & $(+)$ & GATAA & S000199 \\
SORLIP2AT & Light-regulated & 3 & $(-)(+)$ & GGGCC & S000483 \\
TBOXATGAPB & Light-regulated & 1 & $(-)$ & ACTTTG & S000383 \\
LTRECOREATCOR15 & Low temperature & 1 & $(+)$ & CCGAC & S000153 \\
CACTFTPPCA1 & Mesonse & 7 & $(-)(+)$ & YACT & S000449 Specific \\
GT1GMSCAM4 & Salt-induced element & 4 & $(-)(+)$ & GAAAAA & S000453 \\
CBFHV & Water stress response & 1 & $(+)$ & RYCGAC & S000497 \\
DRECRTCOREAT & Water stress response & 1 & $(+)$ & RCCGAC & S000418 \\
MYB1AT & Water stress response & 3 & $(+)$ & WAACCA & S000408 \\
MYBCORE & Water stress response & 1 & $(-)$ & CNGTTR & S000176 \\
MYCCONSENSUSAT & Water stress response & 2 & $(-)(+)$ & CANNTG & S000407 \\
\hline
\end{tabular}

Intergenic region between At2g35490 and At2g35500

This is the smallest intergenic region (240 bp) studied in this work. As in the previews promoter analysis, the sequence between At2g35490 and At2g35500 has the conserved CAAT and TATA boxes in both positive and negative strands (Table 4.5). Besides this, one cis element that is related to heat stress (HSE) is found in the positive strand at site +28 and one motif related to low temperature (LTR) is located in the negative strand at the site -33 . Furthermore, a high number of light-regulated TFBs were identified in the sequence including the GATABOX, GT1CONSENSUS, IBOX, IBOXCORE, IBOXCORENT, SORLIP2AT, SORLIP5AT and TBOXATGAPB sites (Table 4.6). The sequence has one TFBs associated with salt stress (GT1GMSCAM4) and one with low temperature (LTRE1HVBLT49). Several copies of the NTBBF1ARROLB, SURECOREATSULTR11, ARR1AT, ERELEE4, and WRKY71OS sites were also found, all of them related to different plant hormone regulation.

Table 4.5. List of putative cis-acting regulatory elements present in the putative BDP of At2g35490-At2g35500 (from Plant CARE).

\begin{tabular}{|llccll|}
\hline Site & Organism & Position & Strand & Sequence & Function \\
\hline \multirow{2}{*}{ CAAT-box } & Nicotiana glutinosa & 100 & + & CAAT & Common cis-acting \\
& Nicotiana glutinosa & 231 & + & CAAT & element \\
& Pisum sativum & 166 & - & CAAAT & \\
\hline \multirow{2}{*}{ LTR } & Hordeum vulgare & 33 & - & CCGAAA & Low temperature \\
& response \\
\hline \multirow{2}{*}{ TATA-box } & Brassica napus & 157 & + & ATTATA & \\
& A. thaliana & 159 & - & TATA & Core promoter element \\
& A. thaliana & 158 & - & TATAA & \\
\hline HSE & Brassica oleracea & 28 & + & AAAAAATTTC & Heat stress response \\
\hline
\end{tabular}


Table 4.6. List of putative TFBs present in the putative BDP of At2g35490-At2g35500 (from PLACE database).

\begin{tabular}{|llccll|}
\hline \multicolumn{1}{|c}{ Place ID } & \multicolumn{1}{c}{ Function } & $\begin{array}{c}\text { Copy } \\
\text { number }\end{array}$ & Strand & Sequence & $\begin{array}{c}\text { Place } \\
\text { Accession }\end{array}$ \\
\hline NTBBF1ARROLB & Auxin response & 1 & $(+)$ & ACTTTA & S000273 \\
SURECOREATSULTR11 & Auxin response & 1 & $(-)$ & GAGAC & S000499 \\
CAATBOX1 & Common cis-acting elem. & 2 & $(+)$ & CAAT & S000028 \\
ARR1AT & Cytokinin response & 5 & $(-)(+)$ & NGATT & S000454 \\
DOFCOREZM & Endosperm specific & 4 & $(-)$ & AAAG & S000265 \\
ERELEE4 & Ethylene response & 1 & $(-)$ & AWTTCAAA & S000037 \\
WRKY71OS & Gibberellin specific & 2 & $(+)$ & TGAC & S000447 \\
GATABOX & Light-regulated & 2 & $(-)(+)$ & GATA & S000039 \\
GT1CONSENSUS & Light-regulated & 4 & $(+)$ & GRWAAW & S000198 \\
IBOX & Light-regulated & 1 & $(-)$ & GATAAG & S000124 \\
IBOXCORE & Light-regulated & 1 & $(-)$ & GATAA & S000199 \\
IBOXCORENT & Light-regulated & 1 & $(-)$ & GATAAGR & S0000424 \\
SORLIP2AT & Light-regulated & 2 & $(-)(+)$ & GGGCC & S0000483 \\
SORLIP5AT & Light-regulated & 2 & $(+)$ & GAGTGAG & S000486 \\
TBOXATGAPB & Light-regulated & 1 & $(+)$ & ACTTTG & S000383 \\
LTRE1HVBLT49 & Low temperature response & 1 & $(-)$ & CCGAAA & S000250 \\
CACTFTPPCA1 & Mesophyll Specific & 5 & $(-)(+)$ & YACT & S0000449 \\
GT1GMSCAM4 & Salt-induced element & 1 & $(+)$ & GAAAAA & S0000453 \\
\hline
\end{tabular}

\section{Intergenic region between At1g06460 and At1g06470}

The sequence contained between these genes has $1311 \mathrm{bp}$. Apart from the conserved CAAT and TATA boxes, the analysis of the putative BDP by using PlantCare resulted in different ciselements (Table 4.7). The Box 4, G-box, GATA-motif and MRE elements are all light regulated and are present in both directions of the sequence. Also in this region, low temperature and water stress elements were found as well as hormone-related motifs such as ABRE, TATCbox, and TGA-element. The analysis of the sequence by using the Place tool revealed several light, low temperature, and water stress-regulated TFBs. In addition, two heat responsive TFBs are found in this region, PRECONSCRHSP7OA and CCAATBOX1. The presence of a considerable high number of hormone-regulated TFBs was predicted in the sequence between At1g06460 and At1g06470 as is stated in Table 4.8.

Table 4.7. List of putative cis-acting regulatory elements present in the putative BDP of At1g06460-At1g06470 (from Plant CARE).

\begin{tabular}{|llccll|}
\hline Site & Organism & Position & Strand & Sequence & Function \\
\hline ABRE & A. thaliana & 716 & + & ACGTG & Abscisic acid response \\
\hline ARE & Zea mays & 785 & - & AAACCA & $\begin{array}{l}\text { Cis-acting regulatory } \\
\text { element essential for } \\
\text { the anaerobic induction }\end{array}$ \\
\hline
\end{tabular}




\begin{tabular}{|c|c|c|c|c|c|}
\hline Box 4 & $\begin{array}{l}\text { Petroselinum } \\
\text { crispum } \\
\text { Petroselinum } \\
\text { crispum }\end{array}$ & $\begin{array}{r}264 \\
751 \\
\end{array}$ & $\begin{array}{l}+ \\
-\end{array}$ & $\begin{array}{l}\text { ATTAAT } \\
\text { ATTAAT }\end{array}$ & Light response \\
\hline \multirow{8}{*}{ CAAT-box } & Nicotiana glutinosa & $\begin{array}{r}5 ; 49 ; 218 ; 391 ; \\
739 ; 798 ; 1066\end{array}$ & - & CAAT & \multirow{8}{*}{$\begin{array}{l}\text { Common cis-acting } \\
\text { element }\end{array}$} \\
\hline & Pisum sativum & $85 ; 281 ; 1133$ & + & CAAAT & \\
\hline & A. thaliana & 191 & - & CCAAT & \\
\hline & Nicotiana glutinosa & $\begin{array}{c}316 ; 584 ; 588 \\
641 ; 903\end{array}$ & - & CAAT & \\
\hline & Nicotiana glutinosa & $\begin{array}{c}925 ; 1032 ; 1242 ; \\
1307\end{array}$ & - & САAT & \\
\hline & Pisum sativum & $656 ; 1111$ & - & CAAAT & \\
\hline & A. thaliana & 738 & + & CCAAT & \\
\hline & A. thaliana & 1096 & - & CCAAT & \\
\hline CAT-box & A. thaliana & 91 & - & GCCACT & Meristem expression \\
\hline $\begin{array}{l}\text { CGTCA- } \\
\text { motif }\end{array}$ & Hordeum vulgare & 745 & - & CGTCA & MeJA response \\
\hline G-box & A. thaliana & 715 & + & TACGTG & Light response \\
\hline GATA-motif & Pisum sativum & 504 & - & GATAGGG & Light response \\
\hline LTR & Hordeum vulgare & 1278 & + & CCGAAA & $\begin{array}{l}\text { Low temperature } \\
\text { response }\end{array}$ \\
\hline MBS & A. thaliana & 1258 & - & CAACTG & Water stress response \\
\hline MRE & $\begin{array}{l}\text { Petroselinum } \\
\text { crispum }\end{array}$ & 1052 & - & AACCTAA & Light response \\
\hline \multirow{8}{*}{ TATA-box } & A. thaliana & 186 & - & ccTATAAAaa & \multirow[t]{8}{*}{ Core promoter element } \\
\hline & A. thaliana & 1137 & - & TATA & \\
\hline & A. thaliana & 285 & + & TATA & \\
\hline & Oryza sativa & 788 & - & TACATAAA & \\
\hline & Brassica oleracea & 284 & + & ATATAA & \\
\hline & Brassica oleracea & 1136 & + & ATATAA & \\
\hline & A. thaliana & 726 & - & cсTATAAAаa & \\
\hline & A. thaliana & 1203 & - & TATTTAAA & \\
\hline TATC-box & Oryza sativa & 1078 & - & TATCCCA & Gibberellin response \\
\hline $\begin{array}{l}\text { TC-rich } \\
\text { repeats }\end{array}$ & Nicotiana tabacum & 776 & - & ATTCTCTAAC & Stress response \\
\hline $\begin{array}{l}\text { TCA- } \\
\text { element }\end{array}$ & Nicotiana tabacum & 706 & + & ССАТСTTTTT & Salicylic acid response \\
\hline $\begin{array}{l}\text { TGA- } \\
\text { element }\end{array}$ & Brassica oleracea & 177 & + & AACGAC & Auxin response \\
\hline $\begin{array}{l}\text { TGACG- } \\
\text { motif }\end{array}$ & Hordeum vulgare & 745 & + & TGACG & MeJA response \\
\hline
\end{tabular}

Table 4.8. List of putative TFBs present in the BDP of At1g06460-At1g06470 (from PLACE database).

\begin{tabular}{|llcccc|}
\hline \multicolumn{1}{|c}{ Place ID } & \multicolumn{1}{c}{ Function } & $\begin{array}{c}\text { Copy } \\
\text { number }\end{array}$ & Strand & Sequence & $\begin{array}{c}\text { Place } \\
\text { Accession }\end{array}$ \\
\hline DPBFCOREDCDC3 & $\begin{array}{l}\text { Abscisic acid } \\
\text { response }\end{array}$ & 4 & $(-)(+)$ & ACACNNG & S000292 \\
DRE2COREZMRAB17 & $\begin{array}{l}\text { Abscisic acid } \\
\text { response }\end{array}$ & 1 & $(-)$ & ACCGAC & S000402 \\
CATATGGMSAUR & Auxin response & 2 & $(-)(+)$ & CATATG & S000370 \\
SURECOREATSULTR11 & Auxin response & 1 & $(-)$ & GAGAC & S000499 \\
\hline
\end{tabular}




\begin{tabular}{|c|c|c|c|c|c|}
\hline ASF1MOTIFCAMV & $\begin{array}{l}\text { Auxin, salicylic } \\
\text { acid, light } \\
\text { response }\end{array}$ & 1 & $(+)$ & TGACG & S000024 \\
\hline EVENINGAT & $\begin{array}{l}\text { Circadian control } \\
\text { associated } \\
\text { element }\end{array}$ & 2 & $(-)(+)$ & AAAATATCT & S000385 \\
\hline CAATBOX1 & $\begin{array}{l}\text { Common cis- } \\
\text { acting element }\end{array}$ & 1 & $(-)$ & CCAAT & S000030 \\
\hline ARR1AT & $\begin{array}{l}\text { Cytokinin } \\
\text { response }\end{array}$ & 29 & $(-)(+)$ & NGATT & S000454 \\
\hline CPBCSPOR & $\begin{array}{l}\text { Cytokinin } \\
\text { response }\end{array}$ & 1 & $(+)$ & TATTAG & S000491 \\
\hline DOFCOREZM & $\begin{array}{l}\text { Endosperm } \\
\text { specific }\end{array}$ & 27 & $(-)(+)$ & AAAG & S000265 \\
\hline PYRIMIDINEBOXOSRAMY1A & $\begin{array}{l}\text { Gibberellin } \\
\text { response }\end{array}$ & 2 & $(-)$ & CCTTTT & S000259 \\
\hline WRKY71OS & $\begin{array}{l}\text { Gibberellin } \\
\text { specific }\end{array}$ & 8 & $(-)(+)$ & TGAC & S000447 \\
\hline PRECONSCRHSP70A & Heat response & 1 & $(-)$ & SCGAYNRNNNHD & S000506 \\
\hline CCAATBOX1 & Heat response & 2 & $(-)(+)$ & CCAAT & S000030 \\
\hline GATABOX & Light-regulated & 6 & $(-)(+)$ & GATA & S000039 \\
\hline GT1CONSENSUS & Light-regulated & 12 & $(-)(+)$ & GRWAAW & S000198 \\
\hline IBOX & Light-regulated & 1 & $(-)$ & GATAAG & S000124 \\
\hline IBOXCORE & Light-regulated & 1 & $(-)$ & GATAA & S000199 \\
\hline IBOXCORENT & Light-regulated & 1 & $(-)$ & GATAAGR & S000424 \\
\hline INRNTPSADB & Light-regulated & 3 & $(-)(+)$ & YTCANTYY & S000395 \\
\hline SORLIP2AT & Light-regulated & 3 & $(-)$ & GCCAC & S000482 \\
\hline SORLIP5AT & Light-regulated & 1 & $(-)$ & GAGTGAG & S000486 \\
\hline TBOXATGAPB & Light-regulated & 1 & $(-)$ & ACTTTG & S000383 \\
\hline LTRE1HVBLT49 & $\begin{array}{l}\text { Low temperature } \\
\text { response }\end{array}$ & 1 & $(+)$ & CCGAAA & S000250 \\
\hline LTREATLTI78 & $\begin{array}{l}\text { Low temperature } \\
\text { response }\end{array}$ & 1 & $(-)$ & ACCGACA & S000157 \\
\hline LTRECOREATCOR15 & $\begin{array}{l}\text { Low temperature } \\
\text { response }\end{array}$ & 1 & $(-)$ & CCGAC & S000153 \\
\hline CACTFTPPCA 1 & $\begin{array}{l}\text { Mesophyll } \\
\text { Specific }\end{array}$ & 16 & $(-)(+)$ & YACT & S000449 \\
\hline HЕXMOTIFTAНЗН4 & $\begin{array}{l}\text { Pathogen, salt } \\
\text { response } \\
\text { Pro or }\end{array}$ & 1 & $(-)$ & ACGTCA & S000053 \\
\hline PREATPRODH & $\begin{array}{l}\text { hypoosmolarity } \\
\text { response }\end{array}$ & 1 & $(-)$ & ACTCAT & S000450 \\
\hline GT1GMSCAM4 & $\begin{array}{l}\text { Salt-induced } \\
\text { element }\end{array}$ & 2 & $(-)$ & GAAAAA & S000453 \\
\hline ABRELATERD1 & $\begin{array}{l}\text { Water stress } \\
\text { response }\end{array}$ & 1 & $(+)$ & ACGTG & S000414 \\
\hline ACGTATERD1 & $\begin{array}{l}\text { Water stress } \\
\text { response }\end{array}$ & 4 & $(-)(+)$ & ACGT & S000415 \\
\hline CBFHV & $\begin{array}{l}\text { Water stress } \\
\text { response }\end{array}$ & 1 & $(-)$ & RYCGAC & S000497 \\
\hline DRECRTCOREAT & $\begin{array}{l}\text { Water stress } \\
\text { response }\end{array}$ & 1 & $(-)$ & RCCGAC & S000418 \\
\hline MYB1AT & $\begin{array}{l}\text { Water stress } \\
\text { response }\end{array}$ & 1 & $(-)$ & WAACCA & S000408 \\
\hline MYB2CONSENSUSAT & $\begin{array}{l}\text { Water stress } \\
\text { response }\end{array}$ & 1 & $(-)$ & YAACKG & S000409 \\
\hline MYBCORE & $\begin{array}{l}\text { Water stress } \\
\text { response }\end{array}$ & 1 & $(+)$ & CNGTTR & S000176 \\
\hline
\end{tabular}


To gain more information about the putative BDP, sequences were investigated for the presence of DNase I hypersensitive sites (DHSs) by using the Plant-DHS database [Zhang, 2016]. DHSs are genomic regions that exhibit hypersensitivity to cleavage by DNase I endonucleases. These sites are considered to be open chromatin and cis-regulatory enriched regions in eukaryotic genomes. For the 4 putative BDP studied, specific DHSs with a high score were found upstream of the transcription start sites (TSS) in leaf and flower tissues: TAIRchr4:13083001-13083505, TAIRchr5:20890477-20891503, TAIRchr2:1491364014914117, TAIRchr1:1969299-1969821 (Fig. 4.5, see yellow arrows for DHSs). In addition, there is a depletion of nucleosome occupancy at these sites in the leaf and flower tissues (data not shown). This indicates that the regions are open chromatin sites and thereby have accessibility to transcription factors.

Additionally, the conservation of these putative bidirectional promoters in other species was explored. The conserved arrangement of the genes in 13 other species of dicotyledons was determined with information obtained from the Ensembl Plants database, Plant Compare (Orthologous) [Kersey, 2018]. Homologous genes were considered to be regulated by a conserved BDP (c-BDP) if they were arranged in a bidirectional architecture with the same or with a different gene, and by non-conserved BDP ( $n-B D P)$ when they were regulated by a unidirectional promoter (Table 4.9). Following these criteria, it was found that the putative BDP between At4g25650-At4g25660 and At5g51430-At5g51440 were the most conserved BDP in the other plant species, although the gene pairs were not always conserved together.

Table 4.9. Conservation analysis of putative BDPs.

\begin{tabular}{|c|c|c|c|c|c|c|c|c|}
\hline & At4g25650 & At4g25660 & At5g51430 & At5g51440 & At2g35490 & At2g35500 & At1g06460 & At1g06470 \\
\hline Beta vulgaris & n-BDP & n-BDP & c-BDP & n-BDP & n-BDP & n-BDP & n-BDP & n-BDP \\
\hline $\begin{array}{l}\text { Brassica } \\
\text { napus }\end{array}$ & c-BDP & c-BDP & c-BDP & c-BDP & $\mathrm{n}-\mathrm{BDP}$ & $\mathrm{n}-\mathrm{BDP}$ & c-BDP & c-BDP \\
\hline $\begin{array}{l}\text { Brassica } \\
\text { oleracea }\end{array}$ & c-BDP & c-BDP & c-BDP & c-BDP & n-BDP & n-BDP & c-BDP & c-BDP \\
\hline $\begin{array}{l}\text { Brassica } \\
\text { rapa }\end{array}$ & c-BDP & c-BDP & c-BDP & c-BDP & n-BDP & n-BDP & c-BDP & c-BDP \\
\hline Glycine max & c-BDP & $\mathrm{n}-\mathrm{BDP}$ & c-BDP & c-BDP & $\mathrm{n}-\mathrm{BDP}$ & $\mathrm{n}-\mathrm{BDP}$ & n-BDP & $\mathrm{n}-\mathrm{BDP}$ \\
\hline $\begin{array}{l}\text { Medicago } \\
\text { truncatula }\end{array}$ & c-BDP & n-BDP & c-BDP & c-BDP & n-BDP & n-BDP & n-BDP & n-BDP \\
\hline $\begin{array}{l}\text { Populus } \\
\text { trichocarpa }\end{array}$ & n-BDP & n-BDP & c-BDP & c-BDP & n-BDP & n-BDP & n-BDP & n-BDP \\
\hline $\begin{array}{l}\text { Prunus } \\
\text { persica }\end{array}$ & n-BDP & n-BDP & n-BDP & n-BDP & $\mathrm{n}-\mathrm{BDP}$ & n-BDP & n-BDP & n-BDP \\
\hline $\begin{array}{l}\text { Solanum } \\
\text { lycopersicum }\end{array}$ & c-BDP & $\mathrm{n}-\mathrm{BDP}$ & c-BDP & n-BDP & c-BDP & $\mathrm{n}-\mathrm{BDP}$ & n-BDP & n-BDP \\
\hline $\begin{array}{l}\text { Solanum } \\
\text { tuberosum }\end{array}$ & $\mathrm{n}-\mathrm{BDP}$ & $\mathrm{n}-\mathrm{BDP}$ & c-BDP & $\mathrm{n}-\mathrm{BDP}$ & $\mathrm{n}-\mathrm{BDP}$ & $\mathrm{n}-\mathrm{BDP}$ & $\mathrm{n}-\mathrm{BDP}$ & $\mathrm{n}-\mathrm{BDP}$ \\
\hline $\begin{array}{l}\text { Theobroma } \\
\text { cacao }\end{array}$ & n-BDP & c-BDP & c-BDP & c-BDP & c-BDP & n-BDP & n-BDP & $\mathrm{n}-\mathrm{BDP}$ \\
\hline $\begin{array}{l}\text { Trifolium } \\
\text { pratense }\end{array}$ & c-BDP & n-BDP & c-BDP & c-BDP & c-BDP & n-BDP & n-BDP & n-BDP \\
\hline Vitis vinifera & n-BDP & n-BDP & n-BDP & n-BDP & n-BDP & n-BDP & n-BDP & n-BDP \\
\hline
\end{tabular}



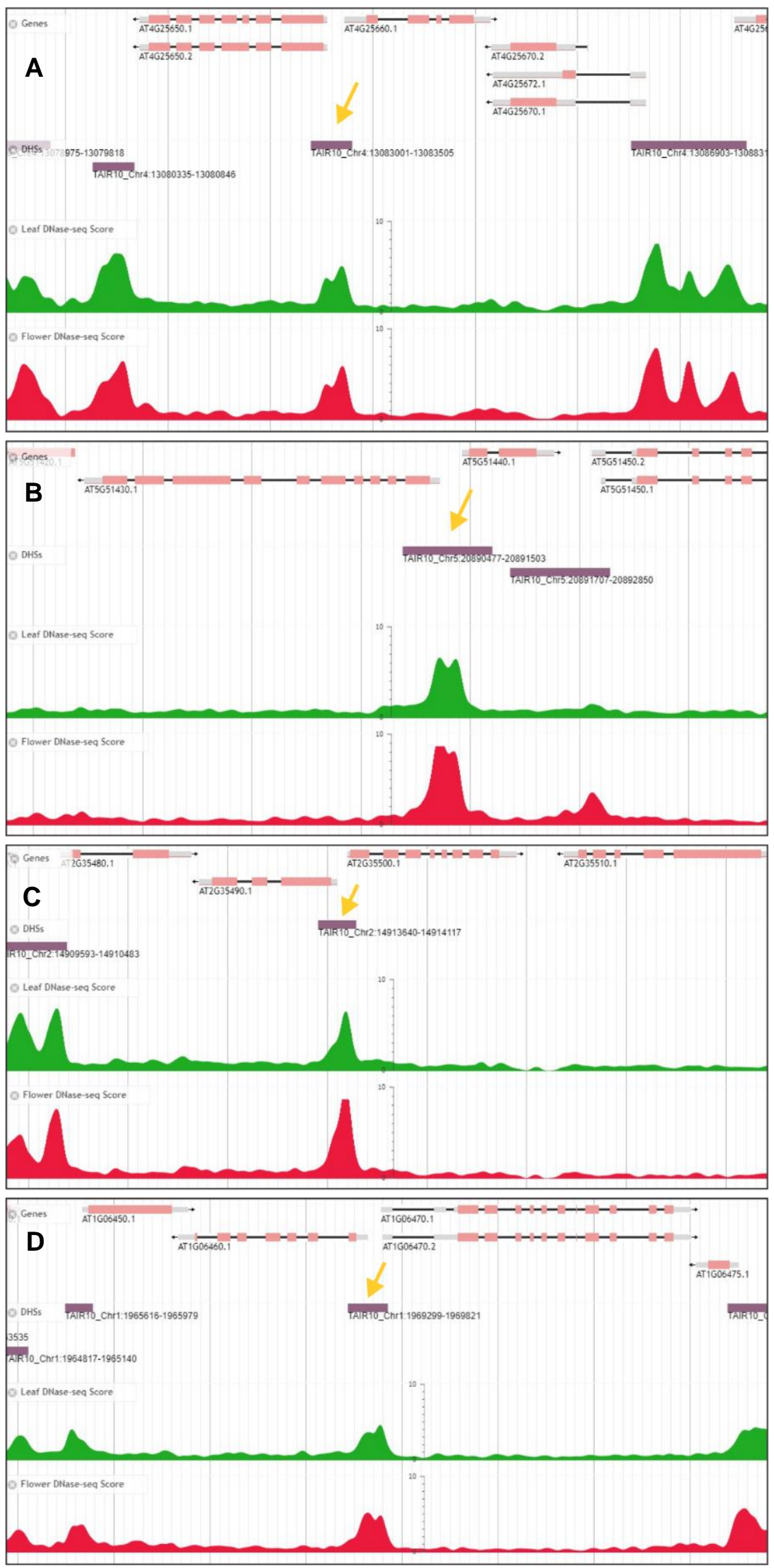

Figura 4.5. Distribution of DNase I hypersensitive sites in the BDPs. DHSs (yellow arrows) were identified by using the Plant-DHS database [Zhang, 2016]. Leaf and flower scores are presented. A) At4g25650-At4g25660,B) At5g51430-At5g51440, C) At2g35490-At2g35500, D) At1g06460-At1g06470. Access 25.01.2018. 


\subsubsection{The activity of the putative bidirectional promoters in A. thaliana seedlings.}

In order to investigate the in vivo activity of the selected BDPs in physiological and stress conditions, the 4 intergenic regions were isolated from the $A$. thaliana genome by PCR. The fragments were then cloned in both directions to a GUS reporter vector (GF9) and the 8 resulting constructs were transformed into Arabidopsis Col-0 plants. For each construct, 5 independent lines were isolated and used in GUS staining experiments. GUS staining was performed in 7 days Arabidopsis seedlings as described in Materials and Methods section. The results obtained from the GUS assays of the transgenic plants indicate that all the intergenic regions showed bidirectional activity (Fig. 4.6). Except for the region between At1g06460 and At1g06470, all the promoter regions showed stronger activity in one direction compared to the other side. The BDP in the par At4g25650-At4g25660 exhibited stronger activity in the PPPDE direction, with expression in leaves and roots. To the other side $(A C D 1-$ like), the promoter activity was lower and only in leaves. Something similar happened for the BDP of At5g51430-At5g51440, where the promoter activity in leaves and roots showed stronger intensity in the SHSP23.5 side. The GUS assays for PromSKL2::GUS gave a strong activity in leaves, while the activity for PromFIB2::GUS was extremely low in the same conditions. The activity of the last BDP located in the middle of At1g06460 and At1g06470 showed comparable intensity in both directions. Further, GUS assays were performed in the transgenic plants exposed to several abiotic stress conditions. The same stress conditions as for the q-PCR experiments were tested, plants were grown under normal conditions and exposed for $1 \mathrm{~h}$ to low temperature $\left(4^{\circ} \mathrm{C}\right)$, and $3 \mathrm{~h}$ to high temperature $\left(37^{\circ} \mathrm{C}\right)$, salinity (150

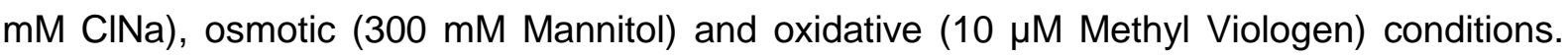
Compared to the control situation, the promoter activity for ACD1-like further accumulated only in cold conditions and it was reduced at high temperature. The activity of the same promoter but in the other direction, showed stronger activity under salt, osmotic and oxidative stresses. The BDPs of EYE and SHSP23.5 had apparently less activity in the osmotic, oxidative, low and high temperature treatments compared to control conditions in the minus strand (in the At5g51430 direction), whereas, in the positive strand (PromsHSP23.5::GUS, At5g51440 direction), the activity had a strong accumulation at high temperature. Interestingly, the activity of the BDPs between FIB2 and SKL2 showed reduced levels under the abiotic stress conditions in both directions. The only special case was the GUS activity in cold conditions for the PromSKL2::GUS. The promoter for At1g06460 showed a particularly increased activity in oxidative and cold treatments and it was slightly reduced in salt and osmotic stresses. No significant changes were observed for this BDP in the other direction (for At1g06470) in the tested conditions. 


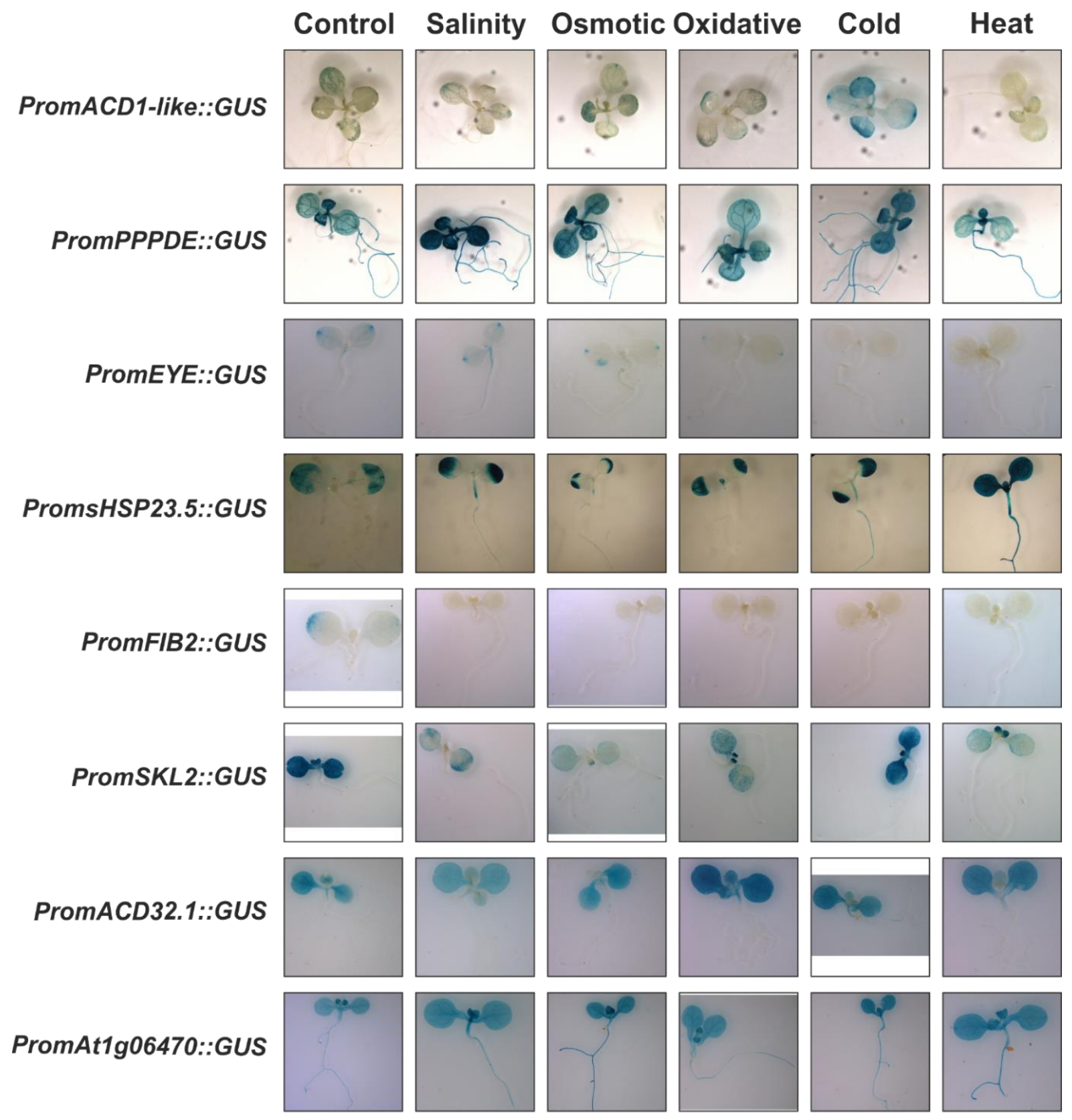

Figure 4.6. Histochemical analysis of GUS activity in $\boldsymbol{A}$. thaliana seedlings. Plants were grown for 7 days under normal conditions and then exposed for $3 \mathrm{~h}$ to high $\left(37^{\circ} \mathrm{C}\right)$ and low $\left(4{ }^{\circ} \mathrm{C}\right)$ temperature, salinity (150 mM CINa), osmotic (300 mM Mannitol) and oxidative (10 $\mu \mathrm{M}$ Methyl Viologen) conditions. GUS staining was performed as stated in Materials and Methods. Five independent transforming lines and a minimum of 20 plants per line were tested for each construct and condition. Pictures correspond to one representative line; comparable pattern staining was found in all the lines of each construct. 


\begin{tabular}{|c|c|c|c|c|c|c|c|c|c|c|}
\hline \multirow[b]{2}{*}{ Gene } & \multicolumn{2}{|c|}{ Salinity } & \multicolumn{2}{|c|}{ Osmotic } & \multicolumn{2}{|c|}{ Oxidative } & \multicolumn{2}{|c|}{ Cold } & \multicolumn{2}{|c|}{ Heat } \\
\hline & $\begin{array}{l}\frac{\pi}{0} \\
\frac{1}{1} \\
\frac{1}{\sigma}\end{array}$ & 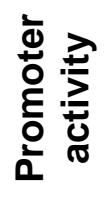 & $\begin{array}{l}\frac{\pi}{0} \\
\frac{1}{\sigma} \\
\text { 이 }\end{array}$ & 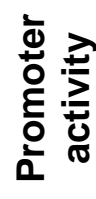 & $\begin{array}{l}\frac{1}{0} \\
\frac{1}{\sigma} \\
\frac{1}{\sigma}\end{array}$ & 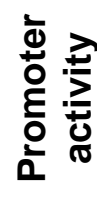 & $\begin{array}{l}\frac{1}{0} \\
\frac{1}{\sigma} \\
\frac{1}{\sigma}\end{array}$ & 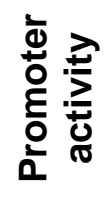 & $\begin{array}{l}\text { 员 } \\
\frac{1}{\sigma} \\
\end{array}$ & 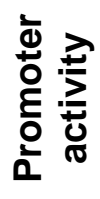 \\
\hline ACD1-like & $\downarrow$ & nd & $\downarrow$ & nd & nsd & nd & nsd & $\uparrow$ & $\downarrow$ & $\downarrow$ \\
\hline PPPDE & $\uparrow$ & $\uparrow$ & $\uparrow$ & $\uparrow$ & $\uparrow$ & $\uparrow$ & nsd & $\uparrow$ & nsd & nd \\
\hline$E Y E$ & nsd & nd & $\downarrow$ & $\downarrow$ & nsd & $\downarrow$ & nsd & $\downarrow$ & nsd & $\downarrow$ \\
\hline HSP23.5 & $\downarrow$ & nd & nsd & nd & nsd & nd & nsd & nd & $\uparrow$ & $\uparrow$ \\
\hline FIB2 & nsd & $\downarrow$ & nsd & $\downarrow$ & nsd & $\downarrow$ & $\uparrow$ & $\downarrow$ & $\uparrow$ & $\downarrow$ \\
\hline SKL2 & nsd & $\downarrow$ & nsd & $\downarrow$ & $\downarrow$ & $\downarrow$ & $\uparrow$ & $\uparrow$ & $\uparrow$ & $\downarrow$ \\
\hline$A C D 32.1$ & $\downarrow$ & $\downarrow$ & nsd & $\downarrow$ & nsd & $\uparrow$ & nsd & $\uparrow$ & $\downarrow$ & nd \\
\hline At1g06470 & $\downarrow$ & nd & $\downarrow$ & nd & nsd & nd & nsd & nd & $\uparrow$ & nd \\
\hline
\end{tabular}

Table 4.10. Summary of genes expression by q-PCR and promoters activity by GUS staining of bidirectional genes. Plants were grown for 7 days under normal conditions and then exposed for $3 \mathrm{~h}$ to high $\left(37^{\circ} \mathrm{C}\right)$ and low $\left(4{ }^{\circ} \mathrm{C}\right)$ temperature, salinity $(150 \mathrm{mM} \mathrm{CINa})$, osmotic (300 mM Mannitol) and oxidative (10 $\mu \mathrm{M}$ Methyl Viologen) conditions. q-PCR and GUS staining experiments were performed as previously described. Arrows indicate increase (up red arrows) and decrease (down blue arrows) in the gene expression or GUS activity. Nd means no qualitative difference in the GUS activity. Nsd means no significant difference in the expression by one-sided t-test. 


\subsubsection{Discussion}

In recent years sequencing technologies made possible the accessibility to more complete genomes and more genetic and genomic resources. This allowed the discovery of particular gene arrangements and promoters in the genome that can be used in the study of plants and crop improvement, to drive specific transgenes. In the genome sequence, different genes arrangements can be found, two neighboring genes can be distributed in a divergent, convergent or parallel configuration [Yang, 2011a]. In the divergent or bidirectional distribution, genes are located in opposite strands of the genome and with head-to-head orientations. The pair of genes arranged in this way is called bidirectional genes, while the intergenic region between them is defined as a bidirectional promoter. This divergent structure has been demonstrated to be quite common in the $A$. thaliana genome, about $13.3 \%$ of the genes are arranged in this distribution [Wang, 2009]. The most probable function of these arrangements is the co-regulation of two adjacent genes by only one promoter. In recent years, bidirectional promoters have received considerable attention because they can initiate transcription in two directions and thus express simultaneously two downstream genes [Wei, 2011; Banerjee, 2013; Wang, 2016].

In this work, the functional activity of four putative bidirectional promoters from Arabidopsis was critically analyzed. The study was not only centered on the characteristics of the intergenic regions but also on the neighbor divergent genes. An initial search for genes coding for ACD proteins which have a divergent orientation with other genes in the $A$. thaliana genome was performed and the pairs At4g25650-At4g25660, At5g51430-At5g51440, At2g35490At2g35500, At1g06460-At1g06470 were selected. In previews genome-wide studies, it has been proposed that bidirectional genes are usually correlated in expression and are involved in similar functions [Wang, 2009]. However, a broad range of functions and expression patterns were observed in the genes considered in this work. The genes in the first pair (At4g25650At4g25660) include a protein that may be implicated in translocation processes in chloroplasts (ACD1-like) and a peptidase located in the cytoplasm (PPPDE). The genes At5g51430 and At5g51440 are both located in organelles (Golgi complex and mitochondria) while the pair At2g35490-At2g35500 codes for chloroplastic proteins, a fibrillin protein and a kinase. Finally, the genes of the fourth pair At1g06460-At1g06470 code for an ACD protein present in peroxisomes and for a transporter protein located in the tonoplast of cells. The expression of the genes in the four pairs was demonstrated by q-PCR to be higher in one direction. Transcripts of At4g256660, At5g51430, At2g35490, and At1g06460 strongly accumulated compared to the level of the correspondent adjacent gene in Col-0 plants. In some pairs, genes displayed similar or opposite responses to the tested abiotic stresses what indicates a common but complex regulation of the genes expression. In Col-0 plants, At4g25650 had reduced expression in salinity, osmotic and heat conditions while the adjacent At4g25660 gene showed 
exactly the opposite response to the same treatments. On the contrary, the expression of the genes in the pair At2g35490 and At2g35500 exhibited the same pattern in low and high temperature, they both accumulated in these conditions. Exclusive expression pattern was also observed for At1g06460 and At1g06470. These genes showed the same expression response to salinity and the opposite to heat stress conditions. To better understand the complexity in the regulation of bi-directional genes, the intergenic regions between them were in silico and in vivo analyzed. The in vivo activity of the four putative BDP was evaluated by successfully cloning these regions in opposite orientation to the $\beta$-glucuronidase (GUS) reporter gene and by exposing the transgenic Arabidopsis plants to various stress situations. The four intergenic regions displayed GUS activity in both directions in all the tested lines proving that they are all indeed bidirectional promoters. That is the same genomic sequence coordinates and initiates the expression of the two divergent genes. Promoter activities were, nevertheless especially different in the positive and in the negative directions, as well as in the tested stresses. In only one BDP, located between At1g06460 and At1g06470, the GUS staining was similar in the 5 independent lines of both contructs and thus the activity could be considered to be divergently the same. In addition to this, the promoter activities tested by GUS staining did not always reflect what was found in the gene expression experiments by qPCR in Col-0 plants (Table 4.10). The activity of the BDP in At4g25650-At4g25660 pair was stronger in the PPPDE direction, as it could be seen from GUS assays and q-PCR experiments. Same patterns were also found for this promoter under abiotic stress conditions. On the contrary, the higher expression of At5g51430 compared to At5g51440 obtained by qPCR could not be reproduced when the activity of this BDP fused to the GUS gene was tested, GUS staining was considerably stronger for the sHSP23.5 strand in all the 5 isolated lines. Both, gene expression of At5g51440 and GUS assays in this direction accumulated under high temperature. The same discordance between q-PCR and GUS experiments was observed for the third BDP, between At2g35490 and At2g35500 genes. The results of genes expression and promoters activities were not always comparable in the tested conditions. The behavior of the BDP::GUS in At1g06460-At1g06470 pair showed no notable changes in response to the abiotic stress treatments, making it difficult the comparison to the q-PCR data. These particular expression patterns found in the genes and promoters suggest that more intricated mechanisms are involved in the bidirectional transcriptional regulation, and further experiments will be necessary to a better understanding of this complexity.

DNase I hypersensitive sites (DHSs) were found in all the four BDPs indicating that this sites of open chromatin may be cis-regulatory enriched and transcriptionally active regions. To identify putative cis-elements involved in the co-regulatory function of the BDPs, the four intergenic regions were investigated using the PlantCare and PLACE databases. This analysis revealed the presence of CAAT and TATA boxes in both strands of the four BDP which have 
an important role in the transcription initiation. Furthermore, numerous stress-related elements were located in the identified BDPs. The low responsive LTREATLTI78 and LTRECOREATCOR15 elements were predicted in the minus strand of BDP At4g25650At4g25660. In this strand, the promoter GUS activity was stronger under cold treatments. Also, in this strand, one cis- salt related element was identified (GT1GMSCAM4), which could be involved in the salt stress response of At4g25650 (a reduction in the expression was observed after $3 \mathrm{~h}$ of $150 \mathrm{mM} \mathrm{CINa}$ ). Special interest has the BDP of At5g51430 and At5g51440 since it regulates the expression of a mitochondrial small heat shock protein. As it was formerly postulated, the expression of SHSPs is transcriptionally regulated by highly conserved ciselements named Heat Shock Element (HSE) [Sun, 2002]. In this BDP, 8 HSE sites were localized in the positive strand, in which the GUS staining and q-PCR experiments showed strong heat response. The intergenic region between At2g35490 and At2g35500 has the LTR cis-element and the LTRE1HVBLT49 binding site in the minus direction. These regulatory motifs may be responsible for the induction of At2g35490 in response to low temperatures. In the opposite strand, heat and salt stress related motifs were allocated to this BDP and could explain the response of At2g35500 to the mentioned stresses. Asides from salt-related ciselements, the sequence of the BDP located between At1g06460 and At1g06470 includes low and high temperatures responsive motifs probably controlling the expression of the divergent genes in this abiotic stress situations. It is worth mentioning the identification of several light regulated elements in the 4 BDP that were analyzed. Additional studies need to be performed in plants exposed to high or low luminosity in order to gain insight into the role of these cisregulatory elements.

The conservation of a bi-directional arrangement in other species suggests that this kind of structure may be beneficial for plants and indicates a possible co-evolution of bi-directional genes and BDP.

Here, 4 different bidirectional promoters in the $A$. thaliana genome were successfully identified and characterized. Among them, the promoter of At1g06460-At1g06470 showed comparable high activity in both directions what represents a useful tool to be used in genetic engineering. The rest of the promoters showed greater strength in one side and can be considered as asymmetric bidirectional promoters. The data suggest that the BDP in the pair At5g51430At5g51440 is strongly heat induced in one direction but not considerably stress induced in the other. These promoters are extremely useful because they can drive the simultaneous expression of two genes and at the same time, they have the potential to induce specifically one of them in a certain condition (high temperature) when it is required. 


\section{CHAPTER II}

\subsection{Functional characterization of mitochondrial small heat shock proteins in Arabidopsis thaliana.}

sHSPs are small molecular chaperones present in all kingdoms of life. The roles of sHSPs have been largely studied in previous reports [Haslbeck, 2015; McLoughlin, 2016; Bernfur, 2017]. Nevertheless, their biological relevance in the response to stress conditions and during plant development has not been elucidated. In addition to this, organellar forms of SHSPs are a unique characteristic of plants with the exception of the mitochondrial sHSP present in Drosophila melanogaster and Toxoplasma gondii [Morrow, 2000; de Miguel, 2005], raising the interest in this kind of SHSPs. In this chapter, functional characterization of the mitochondrial small heat shock proteins under stress conditions and during $A$. thaliana development is presented.

\subsubsection{SHSP23.5, sHSP23.6 and sHSP26.5 are putative mitochondrial sHSPs in $A$. thaliana.}

In formerly phylogenetic studies, two mitochondrial small heat shock proteins were identified in Arabidopsis, sHSP23.5 and sHSP23.6 [Scharf, 2001]. Later, classifications of sHSPs in plants have proposed two distinct mitochondrial-localized subfamilies, called MTI and MTII. In this new categorization, sHSP23.5 and SHSP23.6 proteins are grouped into the MTI family whereas a third mitochondrial small heat shock protein, sHSP26.5, was assigned to the MTII family [Siddique, 2008; Waters, 2013]. More recently, the phylogenetic tree of 91 plant mitochondrial proteins identified these three Arabidopsis sHSP in two subgroups, with sequences of the N-terminal domain more intrinsically disordered, but very similar ACD and C-terminal domains [Jaspard, 2016]. In this study, a first search for mitochondrial small heat shock proteins was done by using the Ensembl database and sequence comparisons of the Plant Compare tool. For this purpose, sHSP23.5 and sHSP23.6 genes were used as a query to identify all possible paralogues in Arabidopsis. Both genes showed sequence homology with sHSP26.5 which belongs to the MTII subfamily and with the chloroplastic At4g27670 (Table 4.11). The homologs genes found for At1g52560 in Arabidopsis were At5g51440, At4g25200 and again At4g27670. These results confirm the three mitochondrial sHSP so far reported. 


\begin{tabular}{lcc}
\hline AT5G51440 - Paralogues & Target \% identity & Query \% identity \\
\hline AT4G25200, mitochondrial & 57.14 & 57.14 \\
AT1G52560, mitochondrial & 21.98 & 24.29 \\
AT4G27670, chloroplastic & 29.07 & 31.43 \\
\hline AT4G25200 - Paralogues & Target \% identity & Query \% identity \\
\hline AT5G51440, mitochondrial & 57.14 & 57.14 \\
AT1G52560, mitochondrial & 22.84 & 25.24 \\
AT4G27670, chloroplastic & 30.40 & 32.86 \\
\hline AT1g52560 - Paralogues & Target \% identity & Query \% identity \\
\hline AT4G25200, mitochondrial & 22.84 & 25.24 \\
AT5G51440, mitochondrial & 21.98 & 24.29 \\
AT4G27670, chloroplastic & 24.57 & 25.11 \\
\hline
\end{tabular}

Table 4.11. Paralogues of mitochondrial small heat shock proteins. Homologs of SHSP-M were identified by the sequence comparison tool from the Ensembl Plants database [Kersey, 2018], and filtered for $A$. thaliana species. Access 18.10.2016.

Protein sequences were aligned using the Clustal2.1 program (EMBL-EBI) and the percentage of identity between them was determined (Table 4.12). sHSP23.5 and sHSP23.6 proteins have 210 aa and share high sequence homology between each other (68.29\% identity). sHSP26.5 with 232 aa presents about $30 \%$ homology with the other two mitochondrial sHSP.

\begin{tabular}{c|ccc} 
& sHSP26.5 & sHSP23.5 & sHSP23.6 \\
\hline sHSP26.5 & 100.00 & 32.99 & 33.51 \\
sHSP23.5 & 32.99 & 100.00 & 68.29 \\
sHSP23.6 & 33.51 & 68.29 & 100.00
\end{tabular}

Table 4.12. Percent identity Matrix. Proteins sequences were aligned and the identity matrix was created by Clustal2.1.

Figure 4.7 represents the sequence alignment of the three proteins by using the CLC Sequence Viewer 7.0.2. In the graph, the percentage of conservation represents the level of conservation at a particular position in the alignment, which is also given by the height of the pink bars. If one position is $100 \%$ conserved in the three sequences, the bar is full height. Middle height bars indicate conserved residues in only two of the three sequences. Middle height bars were found in several positions of the alignment and reflect high conservation between sHSP23.5 and sHSP23.6, but not with sHSP26.5. 


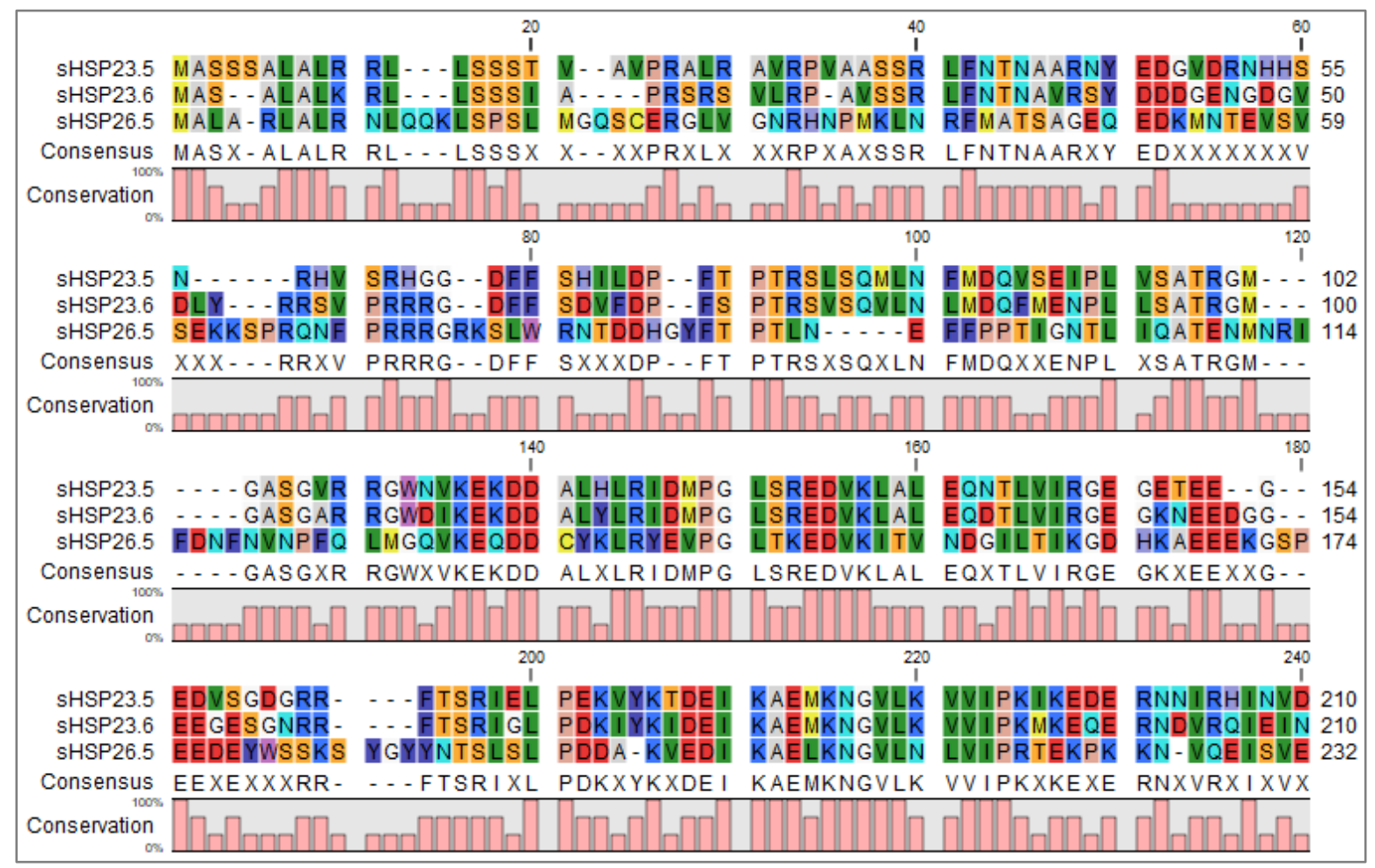

Figure 4.7. Sequence alignment of mitochondrial sHSP. Protein sequences were aligned using the CLC Sequence Viewer 7.0.2 software. Colors were used to distinguish common residues and the consensus indicates amino acid present in at least two sequences. Pink bars represent the percentage of conservation of one position in the alignment.

\subsection{2. $s H S P 23.5, s H S P 23.6$ and $s H S P 26.5$ are regulated by abiotic stresses.}

Considering that most of the small heat shock proteins are heat inducible [Waters, 2013], a quantitative analysis of the sHSP23.5, sHSP23.6 and sHSP26.5 expressions in A. thaliana plants after heat shock was performed by q-PCR. Additionally, other stressful conditions were applied to the plants to evaluate the expression of the three genes in these abiotic stresses. The q-PCR experiments were performed with cDNA, obtained from RNA of whole seedlings. Plants were grown for 7 days under normal conditions and then exposed to different treatments: low and high temperature ( $1 \mathrm{~h}$ at $4{ }^{\circ} \mathrm{C}$ and $3 \mathrm{~h}$ at $37^{\circ} \mathrm{C}$, respectively), salinity ( $3 \mathrm{~h}$ to $150 \mathrm{mM} \mathrm{CINa}$ ), osmotic ( $3 \mathrm{~h}$ to $300 \mathrm{mM}$ Mannitol) and oxidative ( $3 \mathrm{~h}$ to $10 \mu \mathrm{M}$ Methyl Viologen) conditions. For the heat treatment, seedlings were incubated $3 \mathrm{~h}$ at high temperature $\left(37^{\circ} \mathrm{C}\right)$ followed by $1 \mathrm{~h}$ of recovery in the climate chamber at normal growth conditions $\left(22^{\circ} \mathrm{C}\right)$. Plants not exposed to the stressful conditions were used as a control. The reference gene, protein phosphatase 2 (PP2a), was used for the quantification since it is a stable expressed gene under development and stressful conditions [Czechowski, 2005]. For each gene, the obtained data were normalized to the basal expression of the respective gene at control conditions. 
After heat treatment, the expression of sHSP23.5, sHSP23.6 and sHSP26.5 showed strong accumulation with more than 400 -fold enrichment, indicating that the three genes are heat responsive (Fig. 4.8). Among them, the sHSP26.5 exhibited the highest expression after heat, followed by SHSP23.6. Concerning the other stressful conditions, statistically significant differences were observed in the expression of the genes in two cases: a reduction in the number of transcripts of $S H S P 23.5$ under salinity and an increase of $s H S P 26.5$ expression at low temperature compared to control conditions.

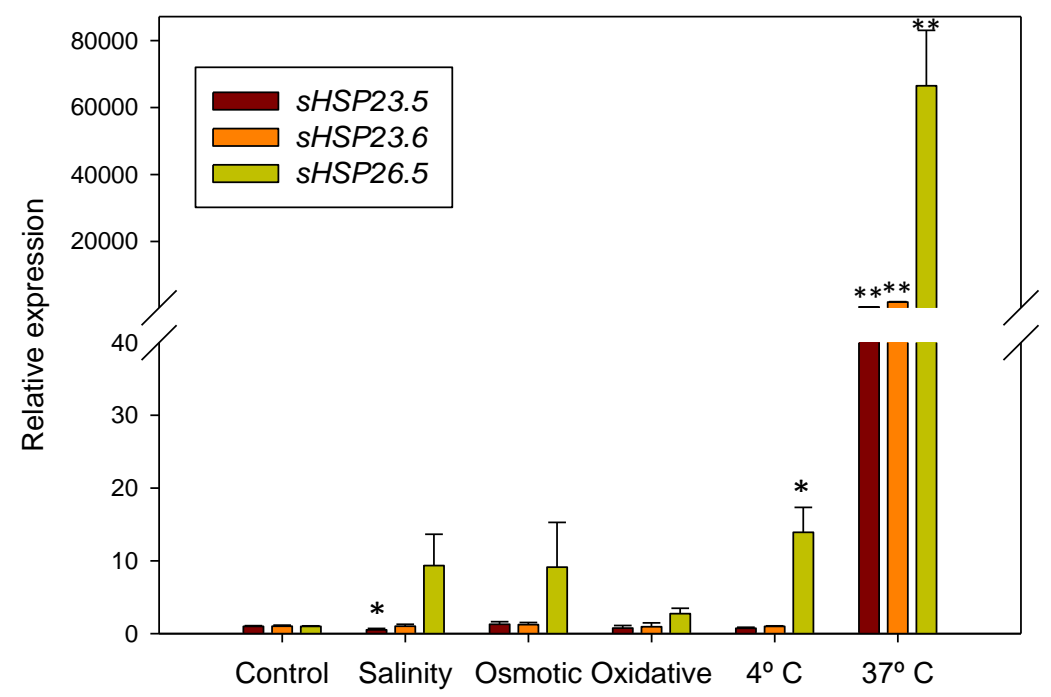

Figure 4.8. Expression of sHSP23.5, sHSP23.6, and $s H S P 26.5$ in $A$. thaliana seedlings. Quantitative real-time PCR of $s H S P 23.5, s H S P 23.6$, and SHSP26.5 was performed after salt, osmotic, oxidative, low and high temperature. Plants were grown for 7 days at $22^{\circ} \mathrm{C}$ under long day conditions and then exposed to different treatments. Experiments were performed twice with comparable results. All expression values were first normalized to the PP2A expression taken as a reference. Relative expression of the transcripts was then normalized to their expression levels at control conditions. Each data point consists of the mean value $+S D$ of four biological replicates obtained in one representative experiment. Twenty plants were pooled for one replicate. Asterisks indicate significance by one-sided ttest with ${ }^{*} p<0.05,{ }^{* *} p<.0 .01$.

The promoter activity of the three genes was also analyzed in the same physiological and stressful conditions as for the q-PCR experiments. Promoter regions of sHSP23.5, sHSP23.6 and sHSP26.5 were isolated from the $A$. thaliana genome by PCR and cloned to a GUS reporter vector (GF9) which was used to transform Arabidopsis Col-0 plants. For each of the three constructs, 5 independent transgenic lines were isolated. GUS staining was performed in 7 days transforming seedlings as described in Materials and Methods section, and the same treatments of heat, cold, salinity, osmotic and oxidative conditions that were described for the q-PCR assays, were applied. Results obtained from the GUS assays demonstrate the heat induction of the three promoters. As can be observed for the deep blue color in Fig. 4.9, higher GUS activity was found after the heat treatment in all the isolated promoters. The bidirectional 
promoter of SHSP23.5 was further studied in Chapter I. In control and some abiotic conditions, the sHSP23.5 promoter activity mostly localized in the leaves edges whereas after heat treatment it was highly increased and extended to the whole seedling. The low activity of the sHSP23.6 promoter in most of the conditions contrasted with the strong activity after $37^{\circ} \mathrm{C}$ treatment. Unlike sHSP23.5 and sHSP23.6, the GUS activity of the sHSP26.5 promoter was high after heat stress but it was found exclusively in roots.

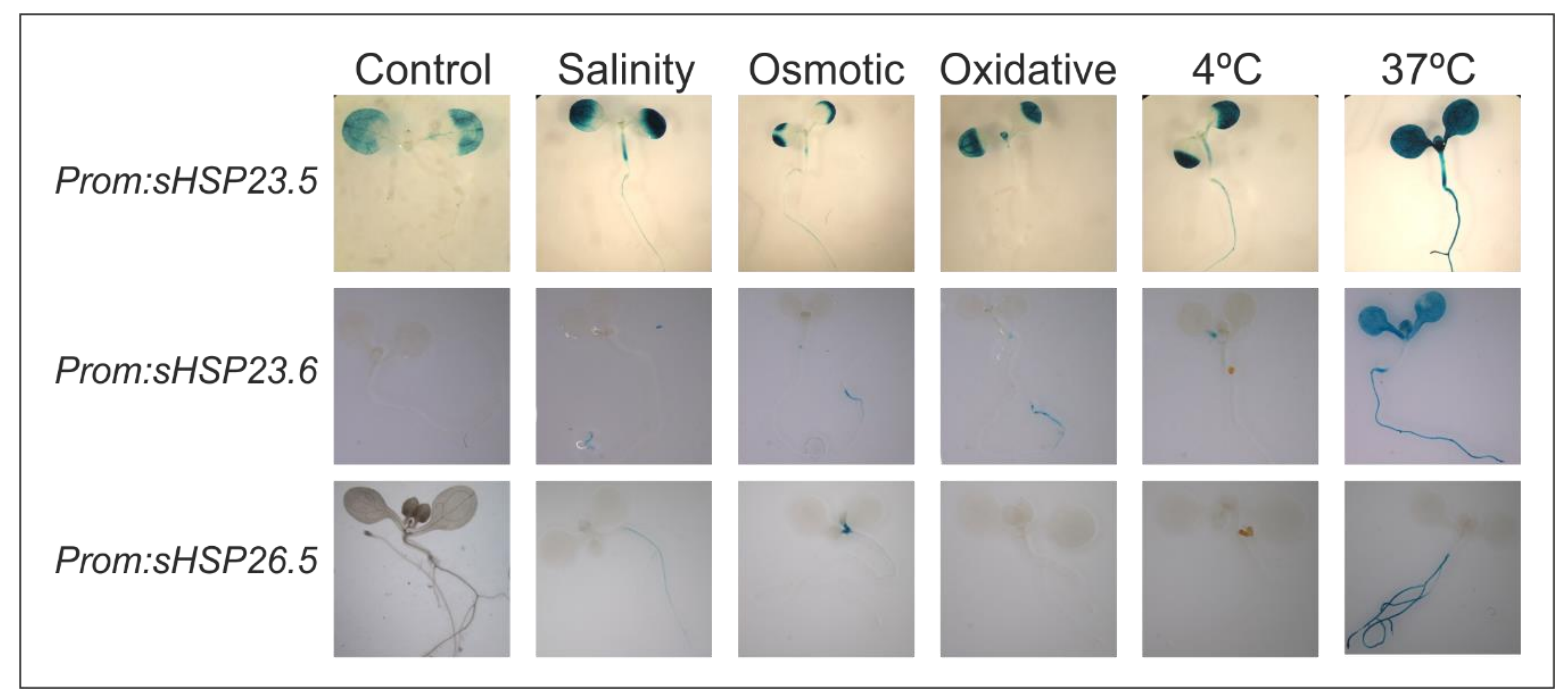

Figure 4.9. Histochemical analysis of GUS activity in $\boldsymbol{A}$. thaliana seedlings. Plants were grown for 7 days under normal conditions and then exposed for $1 \mathrm{~h}$ at low temperature $\left(4^{\circ} \mathrm{C}\right)$, and for $3 \mathrm{~h}$ to high temperature $\left(37^{\circ} \mathrm{C}\right)$, salinity $(150 \mathrm{mM} \mathrm{CINa})$, osmotic (300 mM Mannitol) and oxidative (10 $\mu \mathrm{M}$ Methyl Viologen) conditions. GUS staining was performed as stated in Materials and Methods. Five independent transformed lines and a minimum of 20 plants per line were tested for each construct and condition. Pictures correspond to one representative line; comparable patterns staining were found in all the lines of each construct.

As the relative expression of sHSP23.5, sHSP23.6, and sHSP26.5 under heat stress in $A$. thaliana seedlings were more pronounced, heat-related transcription factor binding sites common to the three promoter sequences were analyzed. The Common TFs tool available in the Genomatix software suite v3.10 [Cartharius, 2005] was used. The search was done against the Matrix Family Library Version 11.0 (September 2017) - A. thaliana, selected groups of Plants and General Core Promoter Elements, a core similarity of 0.75 and optimized matrix similarity. A sequence of $1100 \mathrm{bp}$ was chosen for the At1g52560 promoter. The search identified different motifs of the HEAT family in several positions of the promoters $(p<0.05)$ (Fig. 4.10). These cis- elements may be important for the gene regulation and expression at high temperature. 


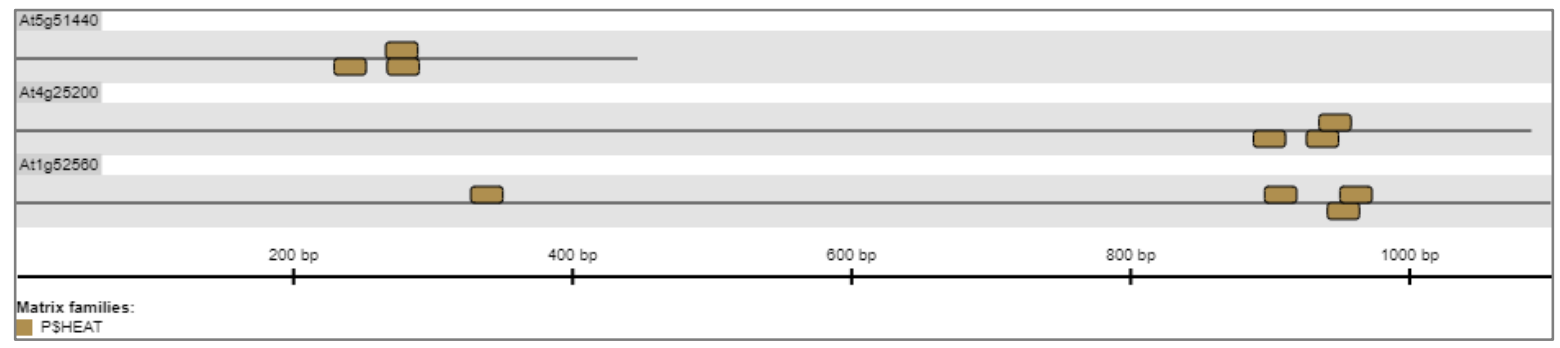

Figure 4.10. HEAT transcription factor binding sites in promoters of mitochondrial sHSPs. Promoter sequences were analyzed for the presence of heat-related transcription factor binding sites by using the Genomatix software suite v3.10 [Cartharius, 2005].

\subsubsection{Artificial microRNAs were designed for the sHSPs-M genes.}

The in vivo functionality of the mitochondrial sHSPs was firstly analyzed in transfer DNA (TDNA) insertion lines of sHSP23.5 and sHSP23.6. However, the available mutants of sHSP23.5 did not show evidently affected phenotype probably because of functional compensation. On the other hand, the homozygous mutation in sHSP23.6 did not affect the abundance of the correspondent gene (data not shown). Considering the possible functional redundancy of the mitochondrial SHSPs, a different strategy was developed, in which the three genes were simultaneously targeted and silenced by an artificial microRNA (amiR) strategy. Triple knockdown mutant plants, from now on refer to as amiR23.5/23.6/26.5 or amiR-T, were obtained by using the design shown in Fig. 4.11. Single knockdown mutants for the individual sHSPs-M (from now on refer to as amiR23.5, amiR23.6, amiR26.5), and a double knockdown mutant for sHSP23.5 and sHSP23.6 (from now on refer to as amiR23.5/23.6) were in parallel generated.
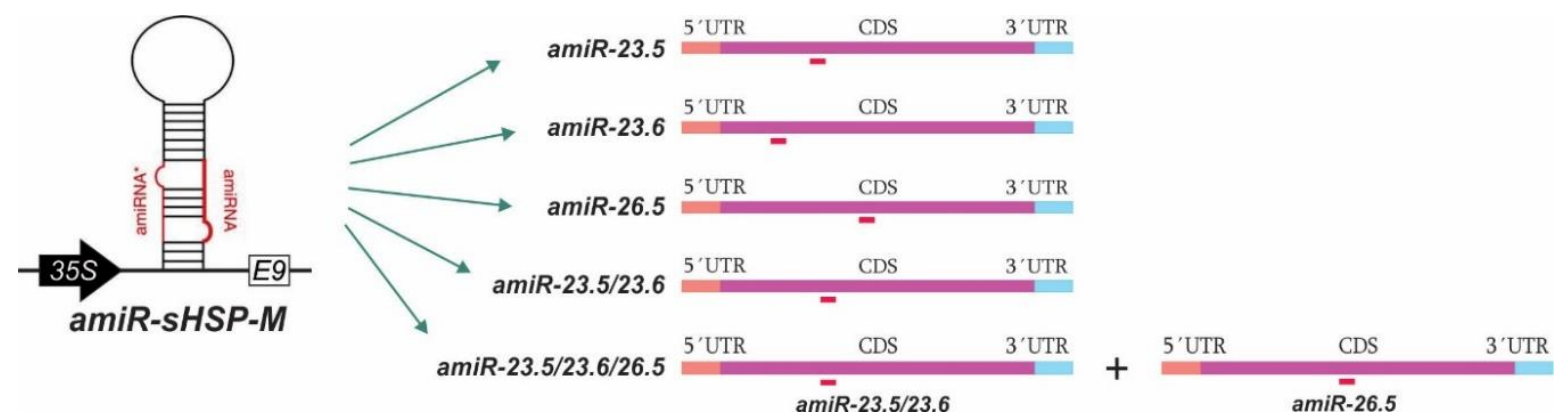

Figure 4.11. Design of amicroRNA targeting SHSP-M. Site-directed mutagenesis and overlapping PCR were used to modify the MIR319a precursor and generate amiR for the sHSPs-M genes. In each construct, amiRs were designed to target a specific sequence (red lines) in the gene transcripts. 
After plant transformation, kanamycin resistant $\mathrm{T} 1$ seedlings were selected and propagated. Approximately 10 independent positive lines were isolated in each construct for further analysis. In order to confirm the expression reduction of the genes that were target of amiRs, a quantitative analysis of the sHSP23.5, sHSP23.6 and sHSP26.5 expressions in A. thaliana transgenics plants was performed by q-PCR. Considering the low basal expression of the three genes under control conditions and that they are all up-regulated by heat (section 4.2.2.), a treatment of $3 \mathrm{~h}$ at $37^{\circ} \mathrm{C}$ was required before the quantification. The q-PCR analysis was performed with cDNA obtained from one leaf of 20 day-old plants. Several T1 plants were screened for the identification of knockdown lines (data not shown). In every single mutant, the expression of individual genes was determined, while in the double mutant lines both sHSP23.5 and sHSP23.6 expression was measured. In the triple mutant lines, the expression of all three genes was quantified. The results indicated that target genes were successfully silenced by the designed amiRs (data not shown). Independent lines with the lowest expression of the target genes were chosen for seed propagation (T2 generation): 4 lines of amiR23.5 and amiR23.6, 3 lines of amiR26.5, 4 lines of amiR23.5/23.6 and 4 lines of amiR23.5/23.6/26.5. All experiments showed in this work were performed with the T2 plants. The reduction in the gene expression of the knockdown mutants compared to the control plants was further confirmed in the T2 plants by q-PCR. In this generation, the analysis was performed with cDNA obtained from RNA of 7 day-old seedlings. These kanamycin-resistant along with Col-0 plants were grown under control conditions and then incubated $3 \mathrm{~h}$ at high temperature $\left(37^{\circ} \mathrm{C}\right)$ to up-regulate the expression. Col-0 plants were used as a control. The reference gene protein phosphatase 2 (PP2a) was added as a stress-unrelated control gene for the quantification. For each gene, data was normalized to the basal expression of the respective gene in Col-0 plants. Fig. 4.12 illustrates the lower expression levels on the genes in the isolated lines silenced by artificial microRNAs, compared to control plants. 


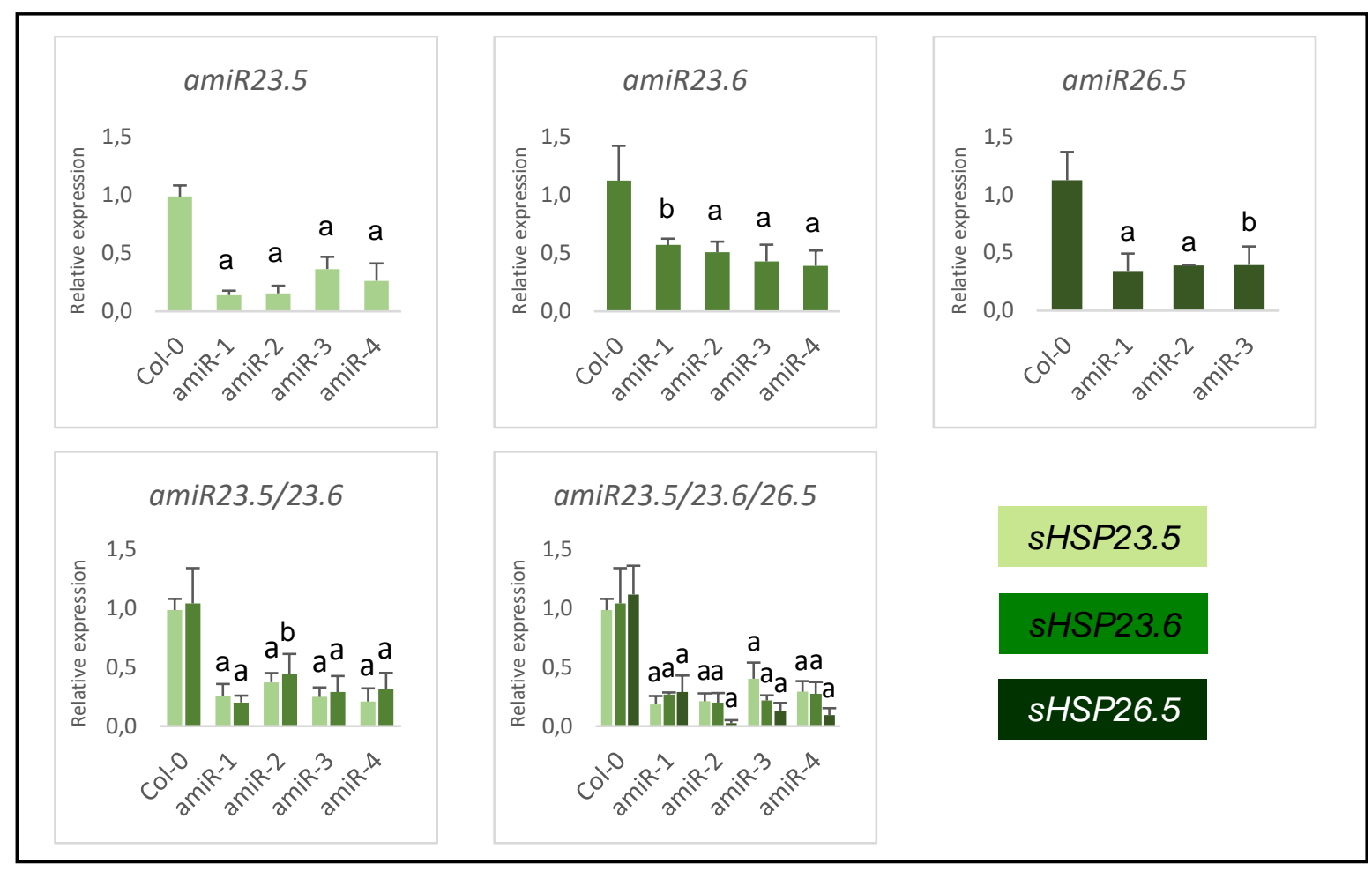

Figure 4.12. Expression of sHSP23.5, sHSP23.6 and sHSP26.5 in knockdown mutants. T2 kanamycin resistant and Col-0 plants were grown for 7 days at $22{ }^{\circ} \mathrm{C}$ under long day conditions and then exposed $3 \mathrm{~h}$ to $37^{\circ} \mathrm{C}$ for expression induction before sampling. Experiments were performed twice and for each construct, 3 to 4 independent transgenic lines were evaluated. All expression values were first normalized to the PP2A expression taken as a reference. Relative expression of the transcripts in the amiR plants was then normalized to their respective expression in Col-0 plants. Each data point corresponds to the mean value $+S D$ of four biological replicates of one experiment. Letters indicate significance by one-sided t-test with $\mathbf{a}: p<.0 .01$ and $\mathbf{b}: p<0.05$.

\subsubsection{Knockdown mutants display altered growth phenotype.}

Mutant plants were phenotypically evaluated during the complete growth cycle in order to find possible effects of the silencing process. Single and double amiR T2 plants did not display severe growth phenotypes. amiR23.5 and amiR23.6 together with the double amiR23.5/23.6 resulted in bigger size plants compared to WT. Seven day-old seedlings and 15 day-old plants of these mutants were equal in size to those of the control Col-0 plants (Fig. 4.13-A). Soon after, 20 day-old plants of the single and double mutants of SHSP23.5 and sHSP23.6 evidently produced bigger rosettes than control plants (Fig. 4.13-B). Figure 4.13-C shows representative 25 day-old rosettes where it is clear the difference in size of these mutants. The same was observed in 28- and 40-day-old plants (Fig. 4.13-D and -E). As can be seen in the lateral view of the plants in Fig. 4.13-E, amiR23.5, amiR23.6, and the double amiR 23.5/23.6 are taller compared to the Col-0 plants. In senescent 55 day-old plants, all amiR lines had the Col-0 size except for the amiR-T (Fig. 4.13-F). The single amiR26.5 lines, on the other hand, developed control-like phenotype. No significant differences were observed at the vegetative and reproductive growth stages in these lines (Fig. 4.13). The most extreme phenotype was found 
in the triple amiR23.5/23.6/26.5 where the complete development was affected. These plants were significantly smaller than Col-0 plants in the complete growth cycle. Although amiR-T mutants developed fully expanded rosettes, their size was considerably smaller as can be observed in Fig. 4.13 and the dwarf growing mutants reached an only half height of the control plants. In addition to the dwarf phenotype and the small size of leaves, amiR-T plants produced narrower leaves than Col-0 plants, and a chlorotic and reticulated phenotype (Fig. 4.13-D and Fig. 4.14-A).

Expanded rosettes of 28 day-old plants were dissected to better evaluate leaves phenotype in mutants (Fig. 4.14-B). Unlike Col-0 plants with leaves that are flat and completely expanded, both single amiR23.5 and amiR23.6 produced curved down leaves. This phenotype was even stronger in the double amiR23.5/23.6 where sHSP23.5 and sHSP23.6 are simultaneously down-regulated. As it was mentioned before, leaves of amiR26.5 did not display any difference to Col-0 plants. It was evident when dissecting rosettes, the small size of the leaves in the amiR-T lines, indicating an alteration in the correct development and growth of these plants. In light of the phenotype observed in the triple knockdown lines, the question of whether the small plant size is due to small cells or to a reduced number of cells, or to both, arose. Leaf cells from amiR-triple and Col-0 plants were observed using differential interference contrast microscopy. For this purpose, second leaves of 21 day-old plants were first incubated with lactic acid to obtain clear epidermal cell views, and the cell number and areas were measured. Besides the reduced leaves areas, epidermal cell areas were significantly smaller in the amiRT. However, the estimated number of epidermal cells per leaf was the same for mutants and Col-0 plants, suggesting that the phenotype in the leaves is mostly due to the smaller cells but not to a less number of them (Fig. 4.15). 


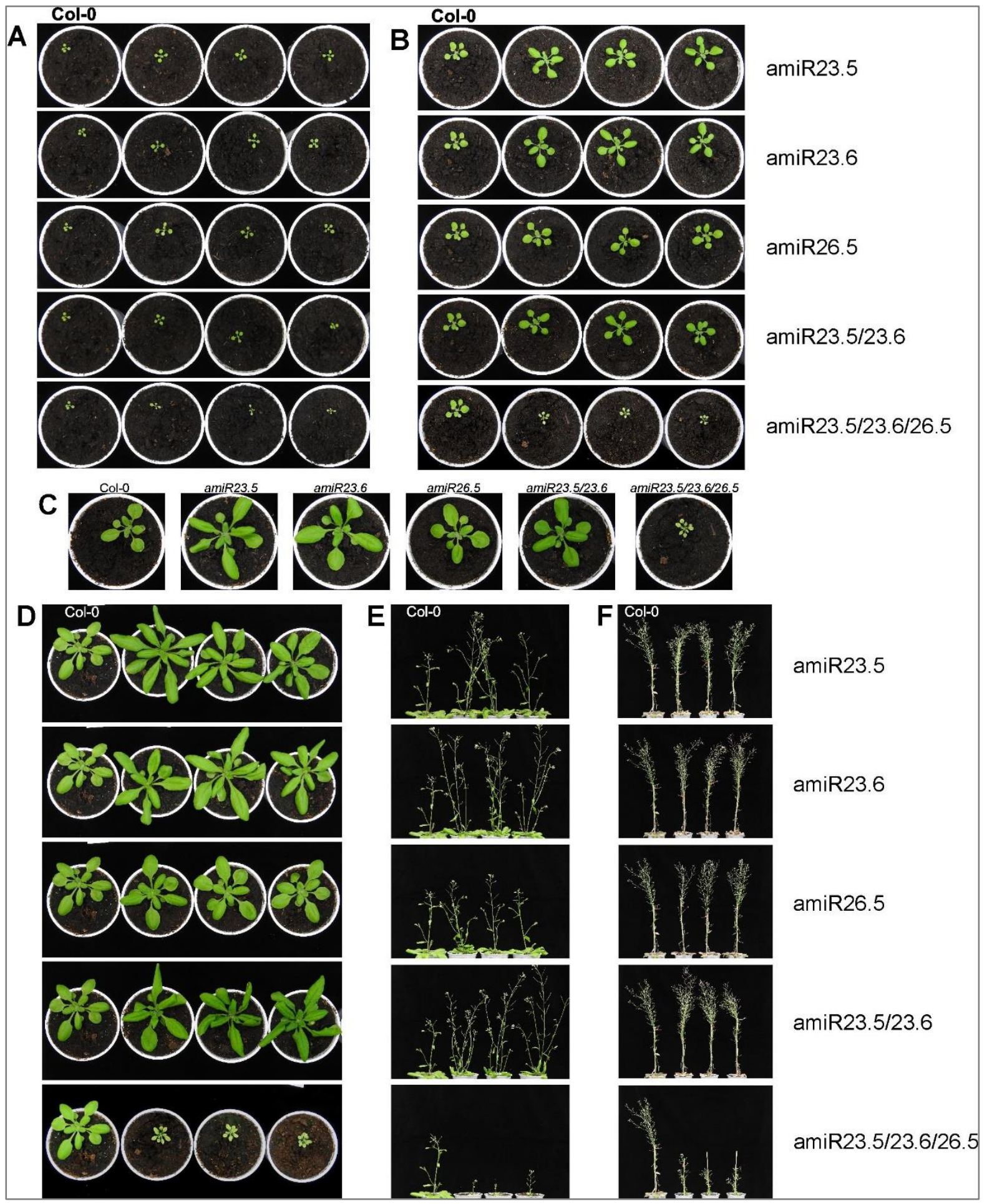

Figure 4.13. Phenotypes of amiR mutants. Plants 15 day- (A), 20 day- (B), 25 day- (C), 28 day- (D), 40 day- $(E)$ and 55 day-old $(F)$. In each picture, from left to right Col-0 plants are followed by representative plants of three independent transgenic lines. 


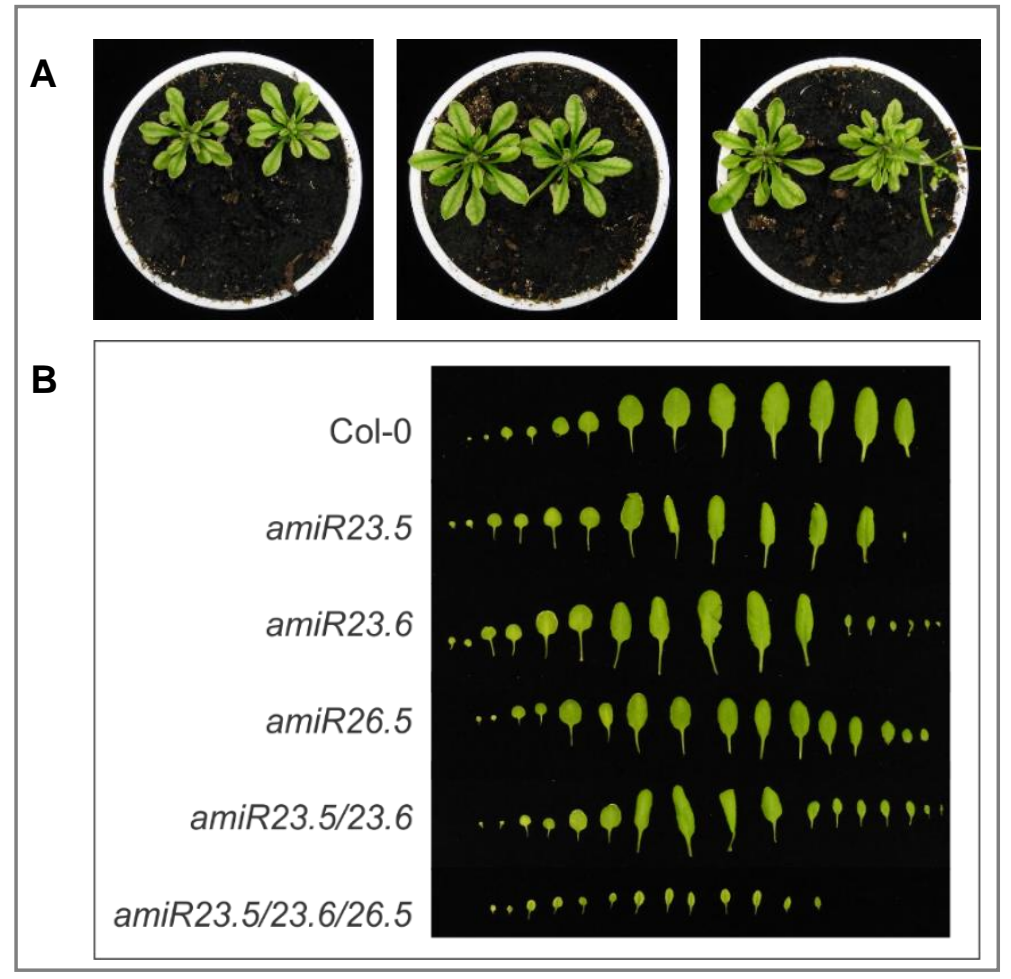

Figure 4.14. (A) View of the small chlorotic leaves of amiR-T mutants. (B) Leaf series of the amiR knockdown mutants. Twenty-eight days-old rosettes were dissected and the individual leaves arranged to generate the series views.

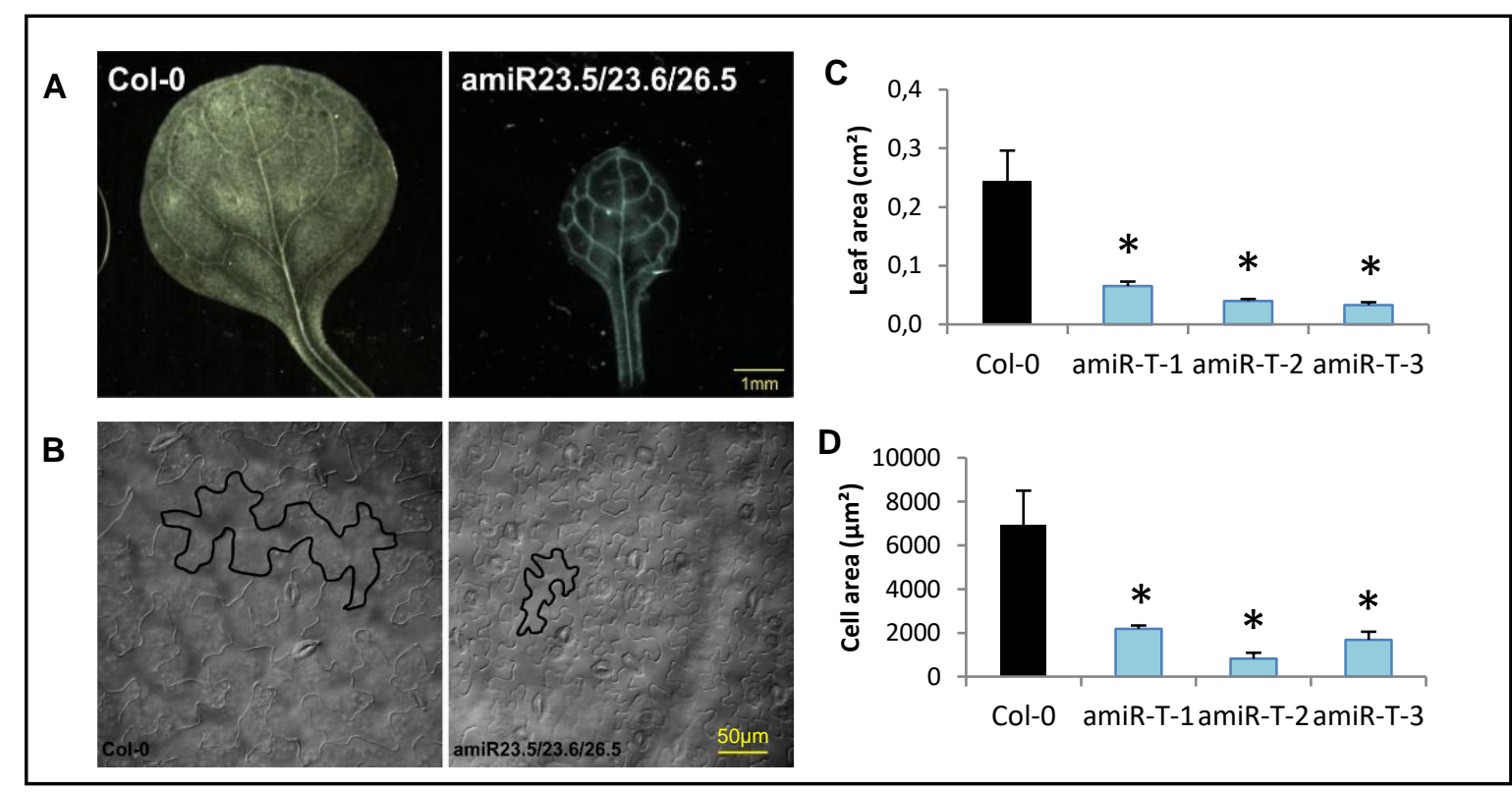

Figure 4.15. Epidermal cell areas of amiR23.5/23.6/26.5 and Col-0 plants. Leaves from 21 day-old plants were incubated with lactic acid at room temperature until tissues were completely cleared and leaf epidermal cells were observed using differential interference contrast microscopy. (A) and (B) are views of leaves and epidermal cells. (C) and (D) are the measured leaf and cell areas, respectively, in Col-0 and three amiR-T independent lines. Data points represent mean areas of 6 leaves and a minimum of 650 cells per transgenic line or Col-0. Asterisks illustrate significance by one-sided t-test with ${ }^{*} p<0.01$. 
Besides the phenotype observed in the vegetative tissues of mutants, including the altered development of the triple amiR23.5/23.6/26.5 and the leaf shapes of two singles and the double amiR23.5/23.6, these plants were investigated for any alteration in the reproductive stages. All mutants showed no differences in the aspect of flowers and siliques compared with Col-0 plants. Nevertheless, seed yield was significantly reduced in the triple knockdown lines (Fig. 4.16).

\section{Seed yield}

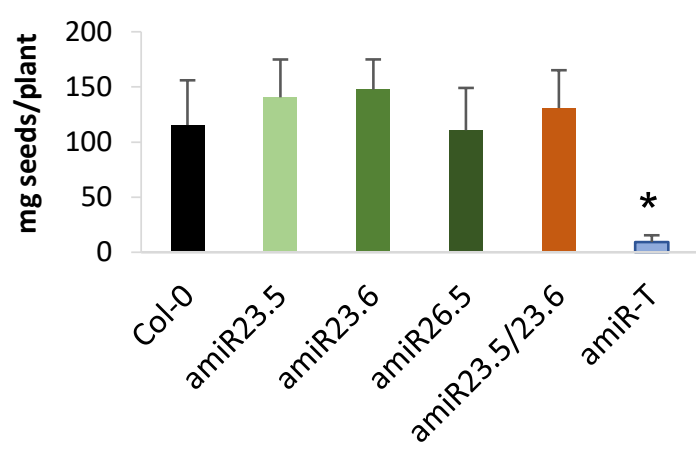

Figure 4.16. Seed production of knockdown mutants. Seeds from senescent plants were collected and weighed. For each mutant, seeds from 3 independent transgenic lines and 3 plants per line were measured. Data points correspond to the mean value + SD of the 3 independent lines. Asterisks mean statistical significance by one-sided t-test with ${ }^{*} p<0.01$.

\subsubsection{Roots as the main altered tissue in amiR26.5 plants.}

Since the three sHSP-M can be expressed in certain circumstances in roots, the growth of these organs was evaluated in the knockdown lines. Transgenic and Col-0 plants were vertically grown under normal conditions for 15 days and the length of the roots was everyday measured. Roots were all similar in aspect and the length of them in amiR23.5, amiR23.6 and amiR23.5/23.6 were the same as in control plants. Surprisingly, amir26.5 produced shorter roots in all the evaluated lines. Roots were also shorter in the 14 independent amiR-T lines that were measured (Fig. 4.17). 


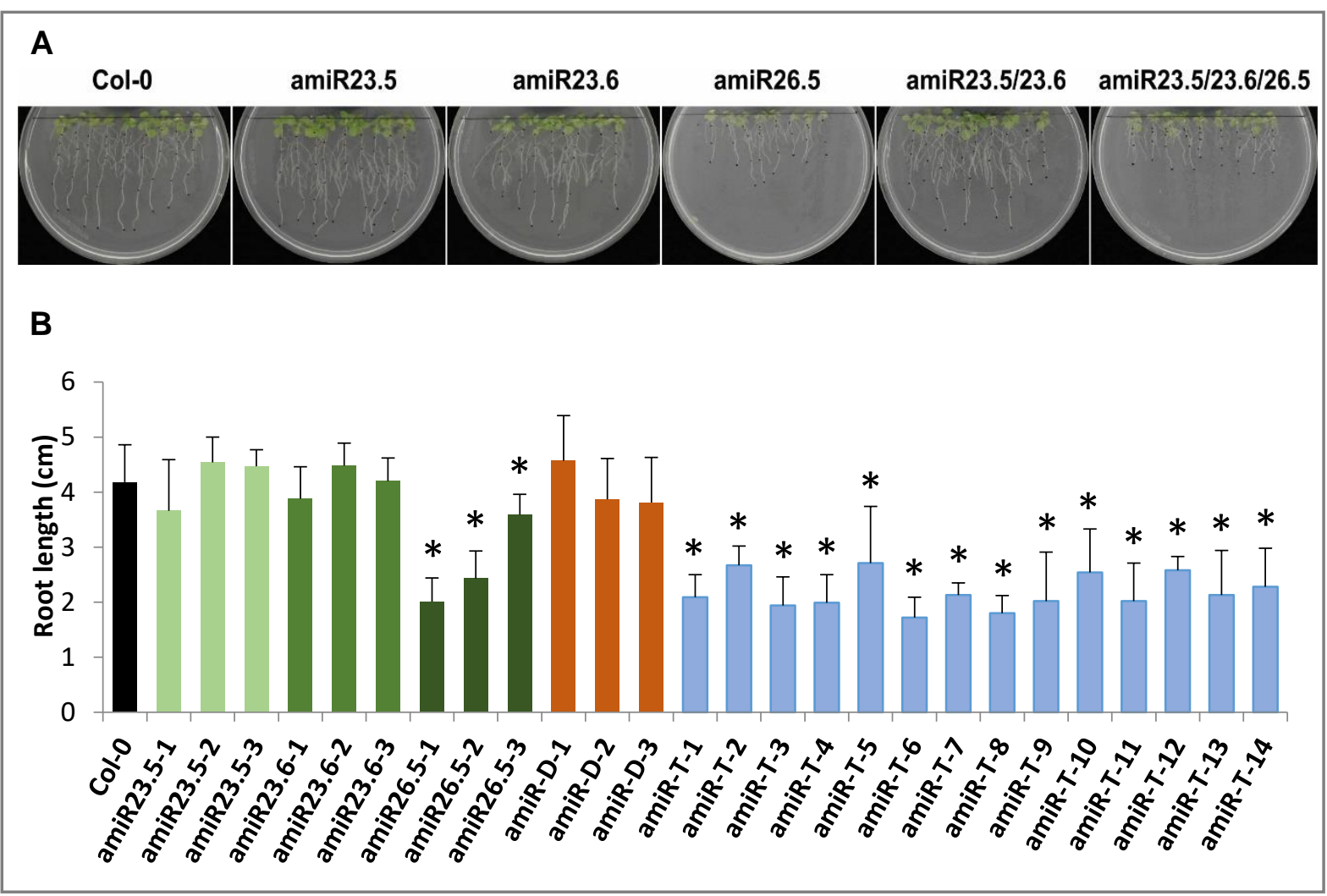

Figure 4.17. Root length of amiR knockdown plants. Plants were vertically grown and roots were periodically photographed and measured. Pictures $(A)$ and values $(B)$ correspond to the length after 15 days of growth. Fourteen independent amiR-T lines were evaluated and three independent lines in the rest of the mutants. Data points represent the mean value + SD of 8 replicates. Asterisks mean significance by one-sided t-test with * $p<0.01$.

\subsubsection{Analysis of knockdown mutants at the protein level.}

To explore the consequences of reduced levels of SHSP-M at the whole-plant level, comparative proteome analyses of control and knockdown mutants was performed. Plants were harvested at a comparable growth stage and differential protein abundances were compared with a comparative quantitative shotgun LCMS analysis. Plants were grown in $1 / 2$ MS under normal conditions for 15 days, a moment in which the samples, which consisted of whole seedlings, were taken. A second experiment of heat-treated samples was carried on in parallel: half of the 15 day-old plants were briefly exposed for $3 \mathrm{~h}$ at $37^{\circ} \mathrm{C}$ followed by $1 \mathrm{~h}$ of recovery at normal conditions before samples were stored. The experiments were based on three biological replicates corresponding to three independent lines of each mutant. Samples consisted of a pool of 50 plants. Besides, two technical replicates of the same experiment were measured. Using shotgun MS/MS-based proteomic analysis 5942 different proteins were identified. The quantitative analysis of the identified proteins was carried out by using the Proteome Discoverer 2.2 ${ }^{\mathrm{TM}}$ software. Protein lists were first assembled according to the groupmaster proteins with high FDR confidence that derive from cross-correlating fragmentation spectra of at least two unique peptides, yielding in a total list of 2405 proteins. For 
quantification, technical and biological replicates were combined and several pair-based comparisons between mutants and controls were done. In each case, abundance ratios were filtered for a fold change cutoff $>2$ and $<0.5$ and a significant $p$-value of less than 0.05 (t-test analysis). In Table 4.13, the number of differentially abundant proteins in the amiR mutants compared to control seedlings is presented. The proteins that showed statistically significant alterations in their abundances between control and amiR plants were selected for further data analysis. They are provided in Supplementary Tables 4.1-4.8.

\begin{tabular}{lccc}
\hline \multicolumn{1}{c}{ Abundance Ratios } & $\begin{array}{c}\text { No of proteins that } \\
\text { significantly changed } \\
(\mathbf{p}<\mathbf{0 . 0 5})\end{array}$ & $\begin{array}{c}\text { Upregulated } \\
\mathbf{\geq} \text { 2-fold }\end{array}$ & $\begin{array}{c}\text { Downregulated } \\
\mathbf{\geq} \text { 2-fold }\end{array}$ \\
\hline amiR23.5/Control & 57 & 51 & 6 \\
amiR23.6/Control & 17 & 9 & 8 \\
amiR26.5/Control & 224 & 217 & 7 \\
amiR23.5/23.6/Control & 243 & 230 & 13 \\
amiR23.5/23.6/26.5/Control & 238 & 227 & 11 \\
amiR23.5/Control-H & 148 & 144 & 4 \\
amiR23.6/Control-H & 25 & 12 & 13 \\
amiR26.5/Control-H & 159 & 150 & 9 \\
amiR23.5/23.6/Control-H & 171 & 156 & 15 \\
amiR23.5/23.6/26.5/Control-H & 145 & 129 & 16 \\
\hline
\end{tabular}

Table 4.13. Number of proteins with differential abundance in the amiRs knockdown compared to control plants. In each comparison, total proteins were filtered for a fold change cutoff $>2$ and $<0.5$ in the abundance ratios and a significant $\mathrm{p}$-value of less than 0.05 by t-test analysis. Letter $\mathrm{H}$ means heat-treated samples.

\subsection{Overview of the proteomes of Arabidopsis amiRsHSP-M plants.}

Comparative analysis of the protein abundances between the amiR and control plants at a fold change cutoff $>2$ and $<0.5$ and a $p<0.05$ revealed that the number of proteins that significantly changed in amiR23.5/23.6 and in amiR-T was larger in comparison to single amiR mutants under normal growing conditions (Table 4.13). When plants were heat-treated, the double amiR23.5/23.6 showed the largest number (171) of proteins significantly changed compared to control plants.

Surprisingly, most of the regulated proteins (around $90 \%$ ) were increasingly abundant in the mutant (Table 4.12). Only in amiR23.6, a comparable number of proteins were up and downregulated in both conditions, normal growth and heat-treated plants. Furthermore, the significantly changed proteome of amiR23.6 was extremely small, 17 and 25 proteins changed in normal and heat conditions, respectively. It must be mentioned that the low number of changed proteins in heat-treated amiR23.6/control ratios is probably due to the low number of total proteins detected in the heat treated-samples of amiR23.6. Fifty-seven proteins showed a change in their abundances in amiR23.5 plants in normal conditions, while in the single 
amiR26.5 and in the double amiR23.5/23.6 and triple mutants, the number of differentially expressed protein was much higher (Table 4.12). This may indicate that sHSP26.5 deficiency, as well as reduced levels of both SHSP23.5 and sHSP23.6, and of all the three sHSP-M, has a broader influence on the Arabidopsis basal proteome. Moreover, when comparing normal and heat stress conditions, the induction of the proteomes in amiR23.5 and amiR23.6 after the treatment is evident. A higher number of proteins with significant fold-change was observed in these treated knockdown mutants. The opposite was found in the treated single amiR26.5, in the amiR23.5/23.6 and in the amiR-T where the number of changed proteins was smaller compared to the number of changed proteins in normal conditions. These data might indicate that the abiotic condition produces a bigger response in the proteome of Arabidopsis plants with deficiency of sHSP23.5 and sHSP23.6 individually.

\subsection{Deficiency of individual sHSPs generates distinct proteome response.}

As it was previously mentioned the number of differentially expressed proteins was especially different in the single mutants amiR23.5, amiR23.6, and amiR26.5. In order to functionally classify the filtered proteins, known and predicted functional associations and gene ontology (GO) annotation according to biological process (BP), molecular function (MF) and cellular compartment (CC) was discovered by using STRING [Szklarczyk, 2015] and Panther [Mi, 2016] databases and software resources.

Panther analyses of the differential proteomes of single amiR23.5, amiR23.6, and amiR26.5 at the $1^{\text {st }}$ level of ontology revealed that these mutants did not substantially differ in the number of detected GO annotations. Nevertheless, some interesting differences were discovered, like the developmental processes found only in amiR23.5 and amiR23.6 mutants but not in amiR26.5 (Fig. 4.18-A), the signal transducer activity term found in amiR23.5 and amiR23.6 mutants, the translation regulator activity term in amiR23.5and amiR26.5 and the presence of an antioxidant activity term exclusively in amiR26.5 (Fig. 4.18-B).

A Venn diagram was used to discover proteins whose abundances were commonly changed in the three single mutants compared to control plants (Fig. 4.19- D and E). Five proteins showed common changes, with three proteins increased while the other two decreased. Among them, changes were observed in three ribosomal proteins located in the cytosol and chloroplasts, one chloroplastic lipoxygenase and one calcium-binding related protein from vacuoles. After heat shock, five common proteins increased in the three single amiR mutants, including one cytosolic ribosomal protein, one nuclear protein belonging to the histone superfamily and one kinase located in the cytosol (see Suppl. Table 4.11 and 4.12). These small numbers of commonly regulated proteins in the three sHSP-M mutants show that their proteomes are quite different and that a deeper analysis should be done individually. 


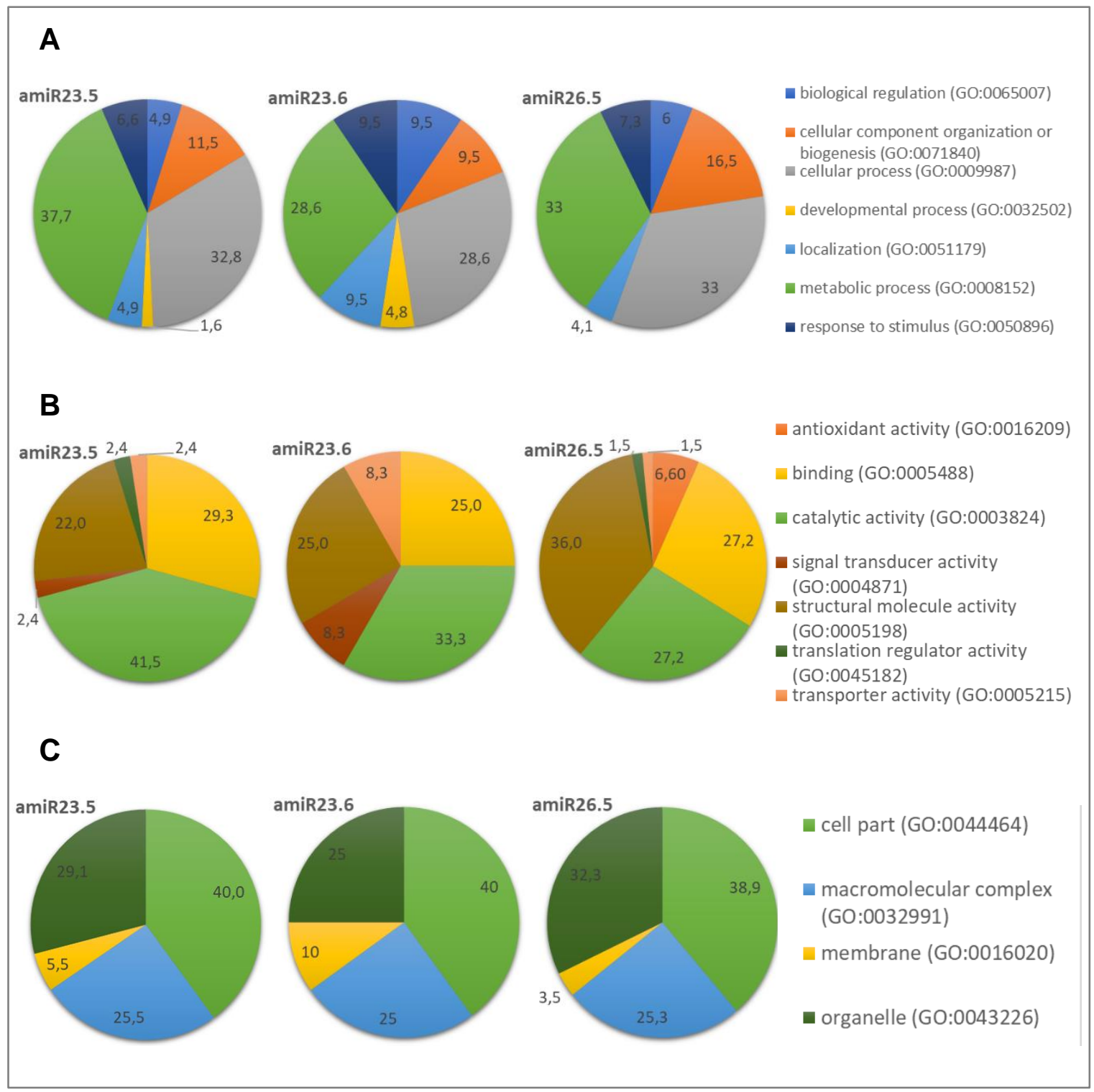

Figure 4.18. Comparison of gene ontology classification by Panther of amiR23.5, amiR23.6 and amiR26.5 mutants in normal conditions of growth according to $(A)$ biological process, (B) molecular function and $(C)$ cellular compartment at the first level of ontology. Access 06.12.18. 


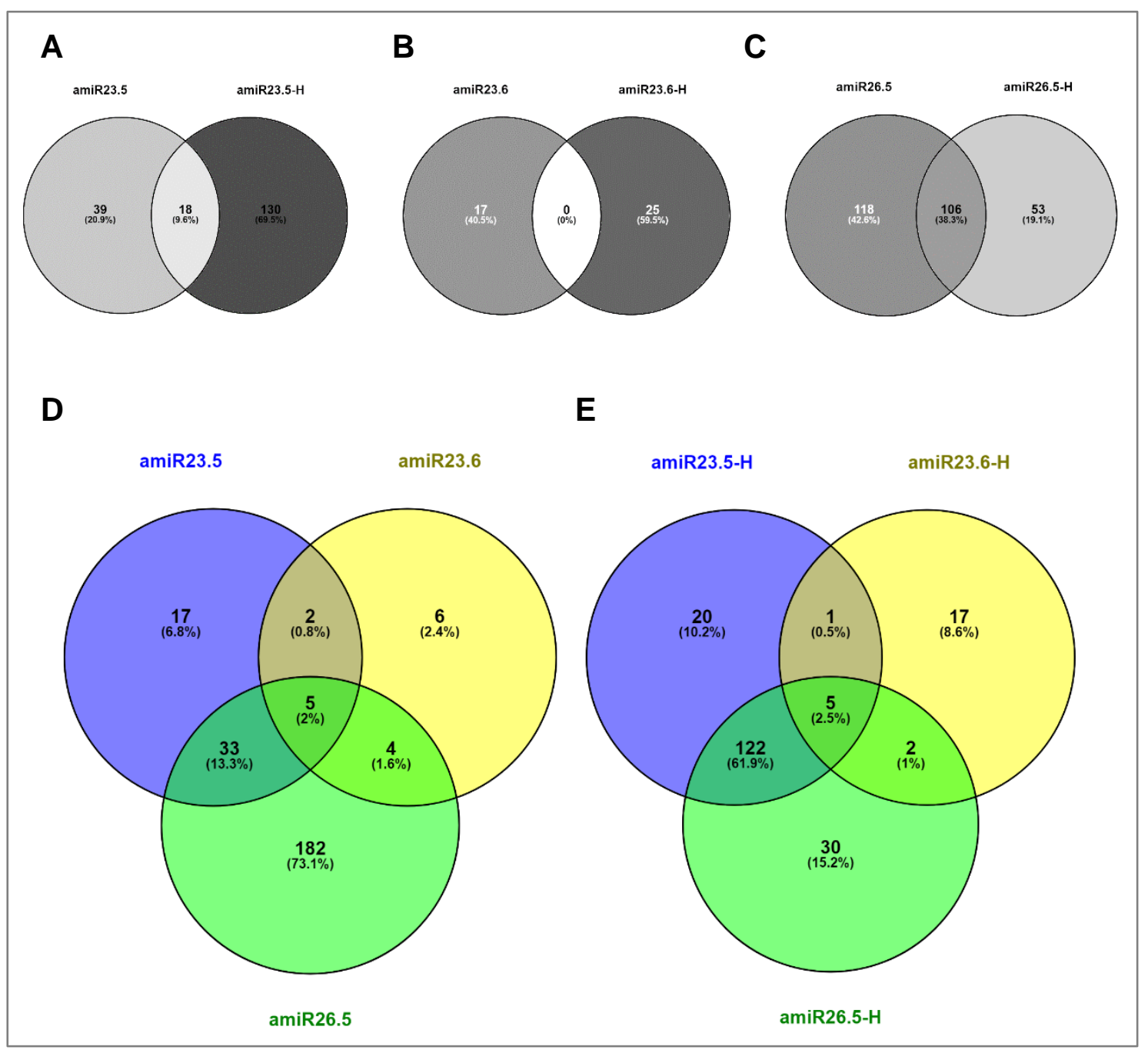

Figure 4.19. Venn diagrams showing numbers of differentially expressed proteins in amiR mutants compared to control plants by using Venny2.1 [Oliveros, 2007]. Number of significantly changed proteins in the normal proteome and in the heat-treated proteome of amiR23.5 (A), amiR23.6 (B) and amiR26.5 (C) mutants. Number of common changed proteins in the three single amiR mutants under normal conditions (D) and after heat stress treatment (E).

In the proteome of amiR23.5, 51 proteins significantly accumulated while only 6 showed reduced levels compare to control plants. Assignment of proteins differing in amount between control and these mutant plants to functional categories was carried out according to STRING. Most of the obtained GO terms in amiR23.5 are related to the biosynthesis of proteins and ribosome biogenesis (Fig. 4.20-blue bars.). Some of the most significant GO terms include translation, organonitrogen compound biosynthetic process, and protein metabolic process. The ribosome compartment (GO:0005840) appeared to be over-represented in the set of proteins, and the term GO:0003735 (structural constituent of ribosome) showed significance at the molecular function level. Interestingly, a cytochrome c related protein (AT1G22840) showed an increase in the mutants (highlighted in Suppl. Table 4.1). The number of proteins with differential expression in amiR23.5 plants was higher after heat treatment, 144 proteins increased and 4 proteins decreased. A Venn diagram was used to exclude proteins that were 
already identified and showed significant change under normal conditions, and a total of 130 proteins from the heat-treated proteome were selected for further analysis (Fig. 4.19- A). The proteins with lower levels include the PGR7 (AT3G21200) that has been proposed to be important in the photosynthetic electron transport of chloroplasts [Jung, 2010] and one plasma membrane $\mathrm{H}^{+}$ATPase (AT5G62670) (highlighted in Suppl. Table 4.6). The STRING analysis of the changed proteins revealed again enrichment of processes related to ribosomes and protein biosynthesis (Fig. 4.20-yellow bars). The protein metabolic process (GO:0043043), translation (GO:0006412), organonitrogen compound biosynthetic process (GO:1901566) are some of the most significant GO terms. Several ribosome-related GO cellular compartment terms were also found. 


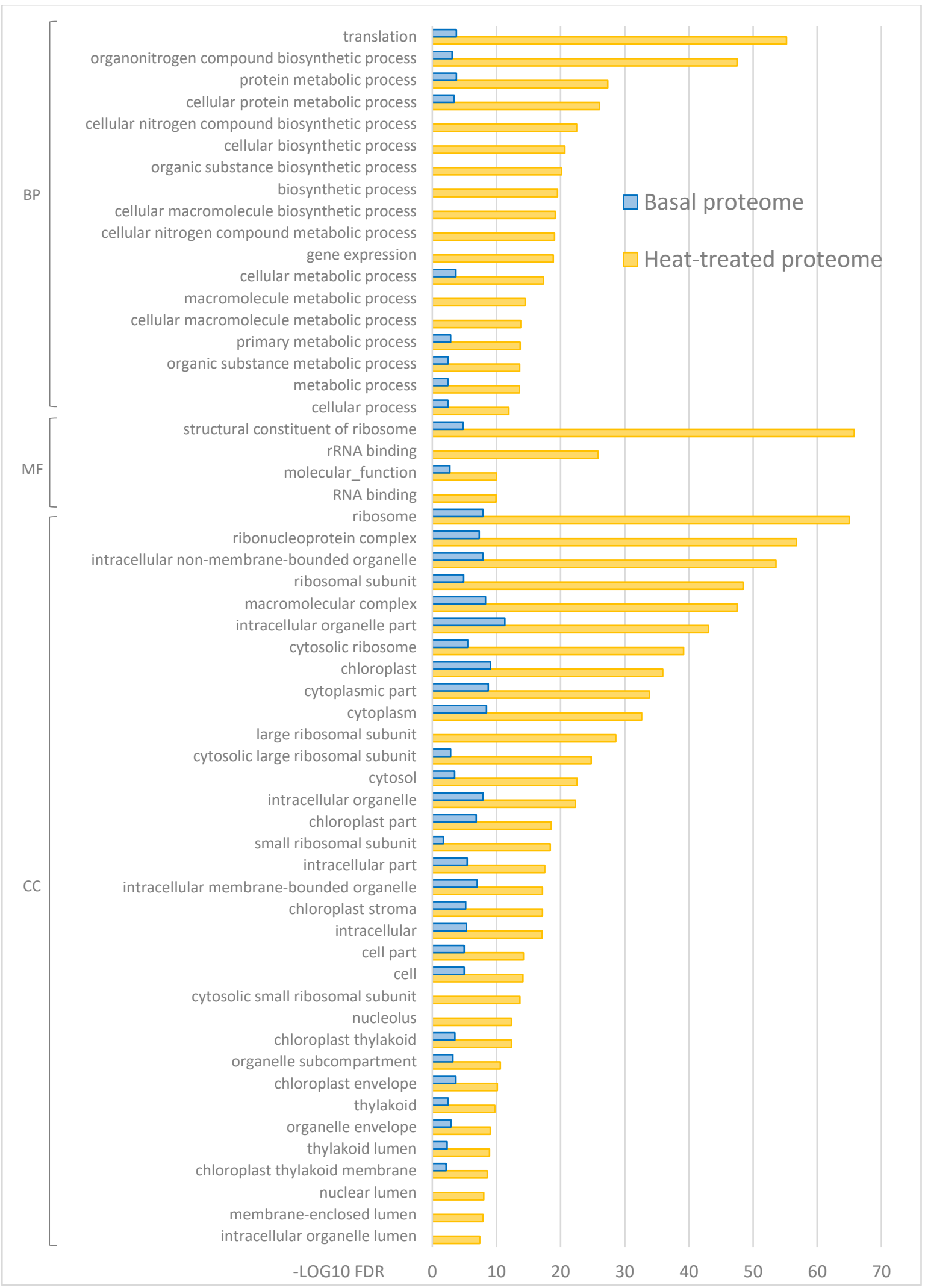

Figure 4.20. GO enrichment of proteins differentially expressed in amiR23.5 relative to control plants in normal and after heat treatment by using STRING. BP: biological process, MF: molecular function, CC: cellular compartment. Access 08.12.18. 
Among the 17 proteins that significantly changed in amiR23.6, 9 proteins increased and 8 decreased. Apart from ribosomal proteins, one purple acid phosphatase PAP2 (AT1G13900) showed higher amount in this mutant than in control plants (highlighted in Suppl. Table 4.2). Like other PAPs, PAP2 can hydrolyze phosphorus compounds and thus it is involved in the acquisition and redistribution of phosphorus in the cell. It has been demonstrated that Arabidopsis transgenic lines overexpressing PAP2 have higher sucrose phosphate synthase (SPS) activity and show increased growth rate and higher seed yield [Sun, 2012]. A lectin-like protein LLP (AT5G03350) involved in salicylic acid (SA)-mediated processes that occur in the effector-triggered immunity (ETI) response [Armijo, 2013], showed also high level in the mutant (highlighted in Suppl. Table 4.2). Similarly, MAM1 (AT5G23010) which is involved in aliphatic glucosinolate biosynthesis [Textor, 2004], significantly accumulated (highlighted in Suppl. Table 4.2). Aliphatic glucosinolates are secondary metabolites that participate in the plant defense. Another increased protein was MKK5 (AT3G21220) that participates in the hydrogen peroxide generation during hypersensitive response-like cell death (highlighted in Suppl. Table 4.2). It has been suggested that prolonged activation of MEKK4 and MEKK5 and the MAPK pathway in cells could lead to the generation of ROS and cell death [Ren, 2002]. The proteins that showed decreased include one aquaporin, TIP1-2 (AT3G26520), one thioredoxin (AT5G65840), a calcium-binding protein-like (AT1G62480), one endosomal protein CHMP1B (AT1G73030) that is required for the plant development [Spitzer, 2009] and the DEAD-Box RNA helicase RH7 (AT5G62190) which is important in the plant growth and development (highlighted in Suppl. Table 4.2). Knockout mutants of $\mathrm{RH} 7$ displayed morphological alterations like disturbed vein pattern, pointed leaves, and short roots, resembling ribosome-related mutants of Arabidopsis [Huang, 2016]. STRING enrichment analysis of the changed proteins resulted in no enriched GO terms in the biological processes and molecular function, while for the cellular components level, plastid-related GO terms showed enrichment (Fig. 4.21-blue bars). Upon heat shock, 12 proteins significantly accumulated and 13 decreased in the amiR23.5 mutants (Suppl. Table 4.7). Proteins of normal and heat shock conditions in this single mutant showed no overlap as it can be observed in Fig. 4.19-B. As it was expected, STRING analysis of the changed proteins revealed enrichment of stress-related GO terms such as response to heat, response to oxidative stress, response to temperature stimulus (Fig. 4.21-yellow bars) 


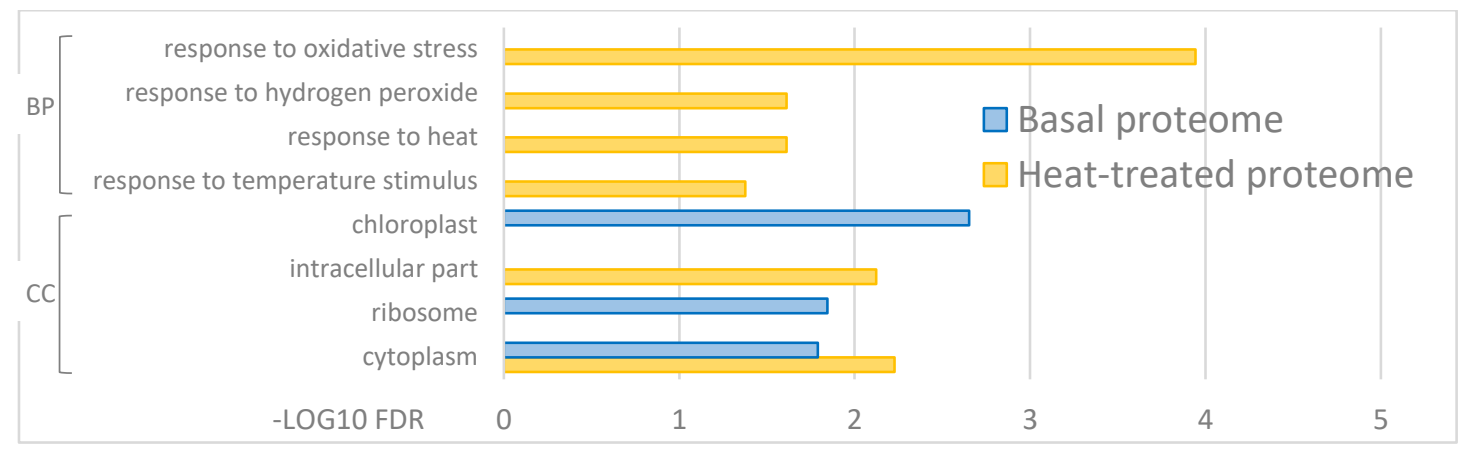

Figure 4.21. GO enrichment of proteins differentially expressed in amiR23.6 relative to control plants in normal and after heat treatment by using STRING. BP: biological process, MF: molecular function, CC: cellular compartment. Access 08.12.18.

The proteome analysis in amiR26.5 identified 217 proteins that significantly accumulated and 7 proteins that decreased in this mutant compared to control plants (Table 4.12). Interestingly, PRXIIF (AT3G06050), GPX6 (AT4G11600) and PRXIIB (AT1G65980), three peroxidases that are important in the cell protection against oxidative stress [Bréhélin, 2003; Finkemeier, 2005], are increased in this mutant (highlighted in Suppl. Table 4.3). The results from STRING analysis gave similar enriched GO terms to those found in the amiR23.5, including translation and cellular protein metabolic process (Fig. 4.22-blue bars). STRING results also searched for the cellular components finding out that the most overrepresented GO terms are ribosome related. When amiR26.5 was exposed to the high-temperature treatment, 150 proteins increased and 9 decreased compare to the proteome of heat-treated control plants (Suppl. Table 4.8). After leaving out the proteins that changed also under normal conditions, the analysis focused on the 53 proteins that specifically changed after the heat shock (Fig. 4.19C). Several GO terms of peptides synthesis and ribosome compartment are enriched in this set of proteins (Fig. 4.22-yellow bars). Among the 8 decreased proteins, RFS2 (AT3G57520) a probable galactosyltransferase involved in the synthesis of raffinose which is found in seeds, roots, and tubers; one plasma membrane $\mathrm{H}+$ ATPase (AT5G62670), and the peroxidase PER16 (AT2G18980), were found (highlighted in Suppl. Table 4.8). 


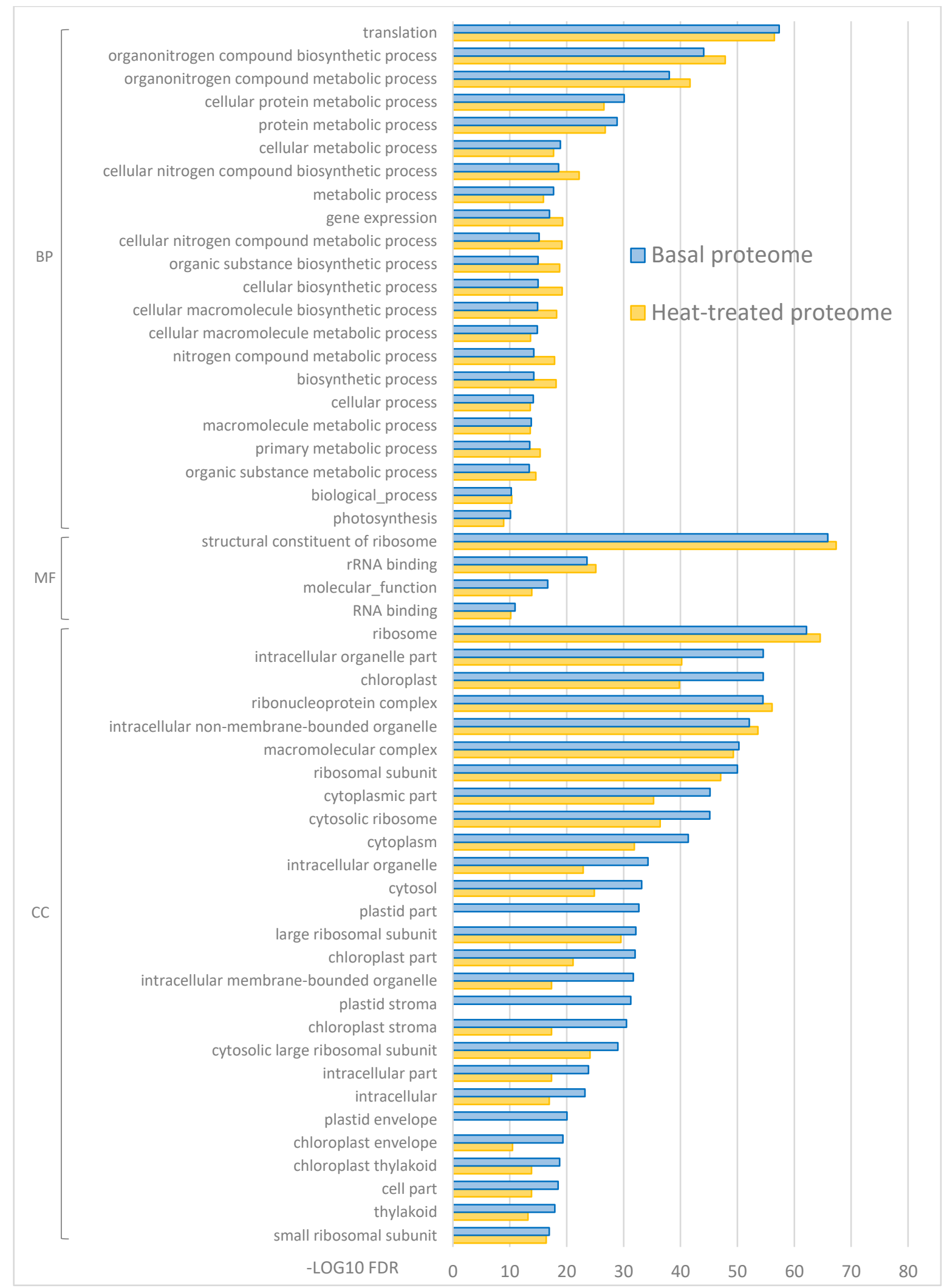

Figure 4.22. GO enrichment of proteins differentially expressed in amiR26.5 relative to control plants in normal and after heat treatment by using STRING. BP: biological process, MF: molecular function, CC: cellular compartment. Access 08.12.18. 


\subsection{Proteome of the double amiR23.5/23.6 mutant widely differs from the proteome of single amiR23.5 and amiR23.6 mutants.}

The differential expressed proteome of the amiR23.5/23.6 included 230 up- and 13 downregulated proteins (Table 4.12). Notably, 201 proteins changed specifically in the double mutant compared to the single mutants amiR23.5 and amiR23.6 (Fig. 4.23-A.). Apart from protein synthesis and ribosome-related GO terms, in this double mutant, the Gene Ontology by STRING analysis resulted in some metabolism-related enriched terms (Fig. 4.24-blue bars). Several proteins that participated in redox reactions and oxidative stress showed accumulation in amiR23.5/23.6 such as the DHAR2 (AT1G75270) that has been proposed to be important in the maintenance of the redox homeostasis by scavenging of ROS under oxidative stresses [Dixon, 2002] (Suppl. Table 4.4). Also, proteins belonging to the thioredoxin superfamily protein, At5g65840, T20H2.2 (AT1G20225), TRXM2 (AT4G03520) and TRXM4 (AT3G15360) (Suppl. Table 4.4). Different peroxidases which play a role in detoxifying peroxides and thus protecting cells against oxidative stress like PRXIIF (AT3G06050), PRXIIB (AT1G65980), GPX6 (AT4G11600) and PRXIIE (AT3G52960) were increased [Bréhélin, 2003; Finkemeier, 2005]. And two superoxide dismutase, CSD2 (AT2G28190) and FSD1 (AT4G25100) (Suppl. Table 4.4). In the heat-treated double mutant, 171 proteins were differentially expressed compared to the heat-treated control plants. From these 171 proteins, 60 changed exclusively after heat shock compared to normal conditions in amiR23.5/23.6 (Fig. 4.23-B). Protein synthesis and translation GO terms are over-represented in this set of proteins. Three HSP2O chaperones increased after the heat shock including the SHSP17.6, sHSP17.8, and SHSP17.4 (Suppl. Table 4.9).

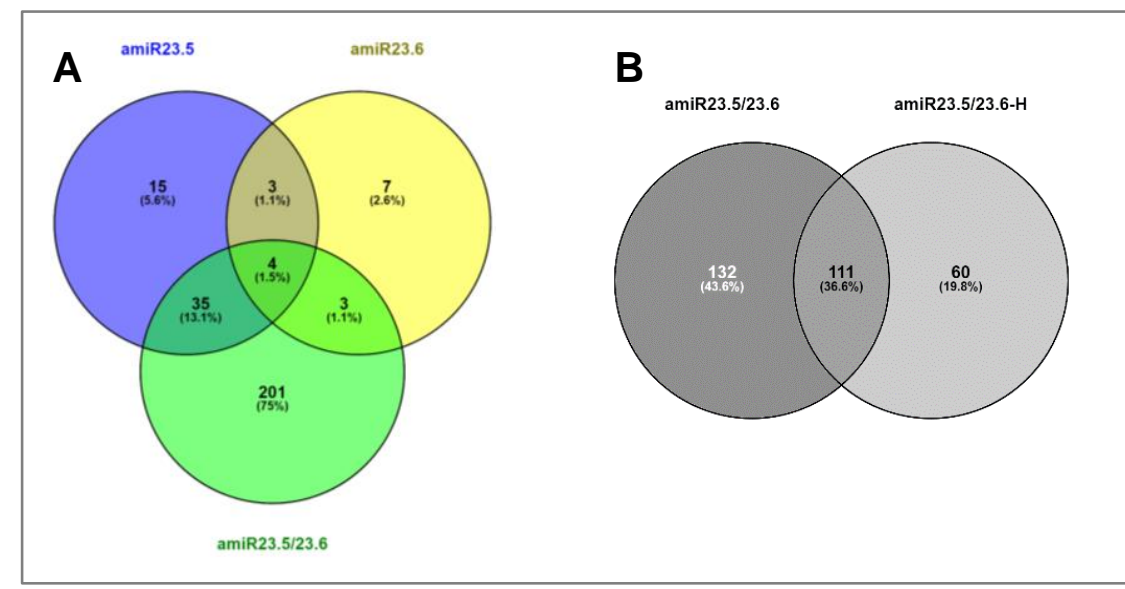

Figure 4.23. Venn diagrams showing numbers of differentially expressed proteins in amiR mutants compared to control plants by using Venny2.1. (A) Number of commonly changed proteins in amiR23.5, amiR23.6, and amiR23.5/23.6. (B) Number of significantly changed proteins in the normal proteome of amiR23.5/23.6 and in the heat-treated proteome. 


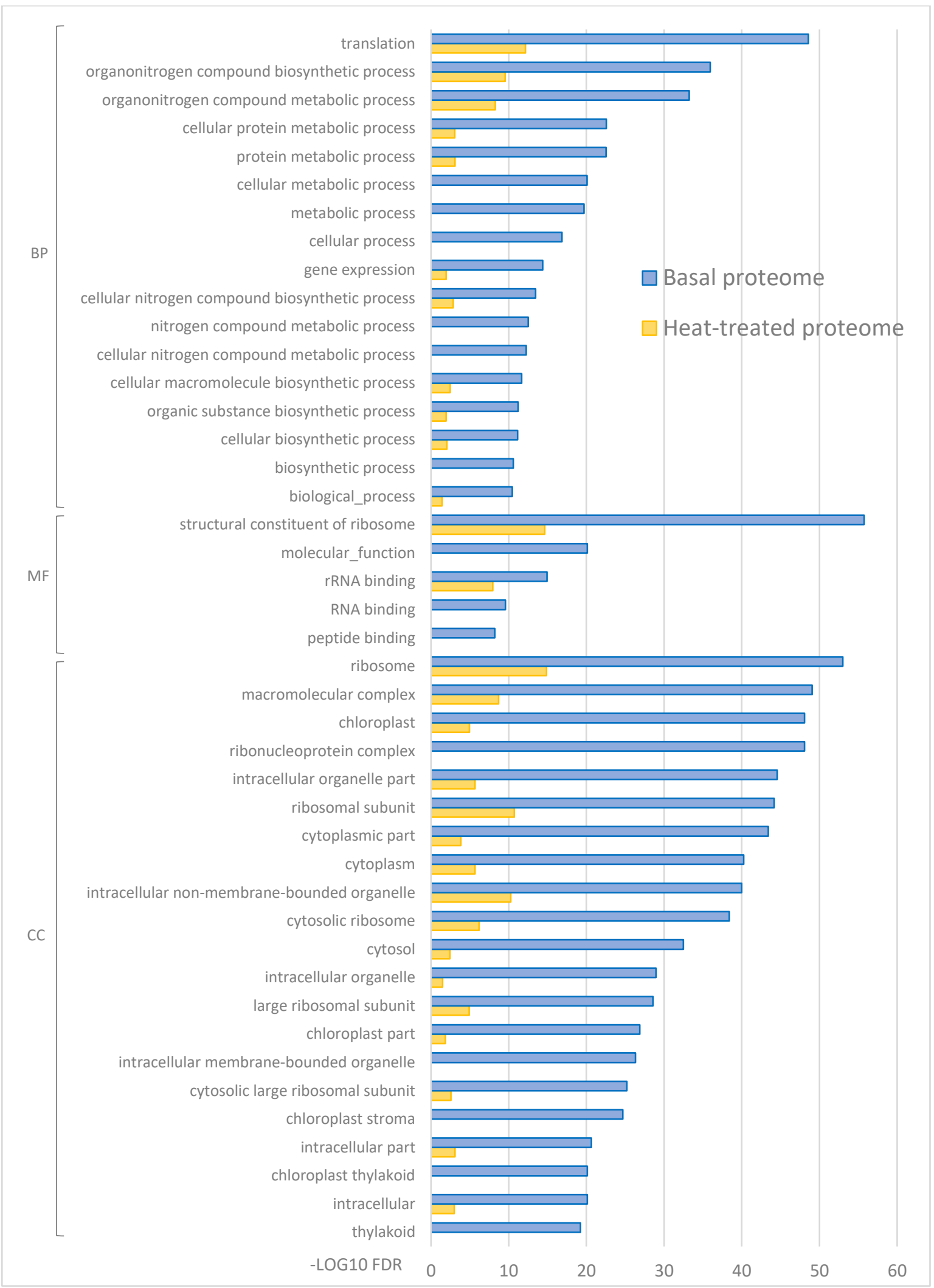

Figure 4.24. GO enrichment of proteins differentially expressed in amiR23.5/23.6 relative to control plants in normal and after heat treatment by using STRING. BP: biological process, MF: molecular function, CC: cellular compartment. Access 08.12.18. 


\subsection{Common changes in the proteome of amiR23.5/23.6/26.5 and single amiR mutants.}

Protein abundances were determined in the triple knockdown mutants under normal conditions and under high temperature. A number of 238 and 145 proteins differentially expressed before and after the heat treatment, respectively. The functional enrichment of the 238 proteins was investigated by STRING (Fig. 4.25). Several protein synthesis-related GO terms and translation were found. Besides this, in the amiR-T mutants metabolism pathways were enriched, especially those related to the primary metabolism. The photosynthesis term showed also over-representation, with 16 protein probably involved in this process (Table 4.15). Venn diagram was carried on with proteins changing in the triple knockdown mutant and in each of the single mutant amiR23.5, amiR23.6, and amiR26.5. The obtained graph shows the overlap of proteins differentially expressed in the mutants and the 42 proteins that changed only in the triple mutant (Fig. 4.26-A). Three photosynthesis-related proteins were exclusively found in the triple mutant with higher abundances: a subunit of the photosystem I PSAE1 (AT4G28750), PSBP1 (AT1G06680) which may be involved in the regulation of photosystem II and the ClpR2 protein (AT1G12410) that is required for the chloroplast development and integrity (highlighted in Suppl. Table 4.5). In addition to these proteins, one protein probably involved in defense response T26J12_10 (AT1G23130) and one thioredoxin AtTrxm1 (AT1G03680) increased in the triple knockdown (highlighted in Suppl. Table 4.5). Among the 42 proteins, the dehydrin ERD14 (AT1G76180) involved in cold and other abiotic stresses response [Kovacs, 2008], DIT2-1 (AT5G64290) which is a glutamate/malate translocator [Renné, 2003], PMDH2 (AT5G09660) a peroxisomal NAD-malate dehydrogenase [Tomaz, 2010] and a serine protease that cleaves phytosulfokines SBTI1.1 (AT1G01900) [Srivastava, 2008], showed lower abundance compared to control plants (highlighted in Suppl. Table 4.5). Most of the GO terms found in the triple amiR in physiological conditions were also present in the proteome of heattreated samples. A set of 52 proteins showed change particularly after the heat shock as it can be observed in a Venn diagram (Fig. 4.26-B). Among these, two peroxidases, PER69 (AT5G64100) and PER16 (AT2G18980) were downregulated after treatment, while two proteins involved in the photosystem I PSAN (AT5G64040) and photosystem II PSB27-1 (AT1G03600) function, were upregulated. Only one HSP-like protein was increased (AT1G54050) (highlighted in Suppl. Table 4.10). 


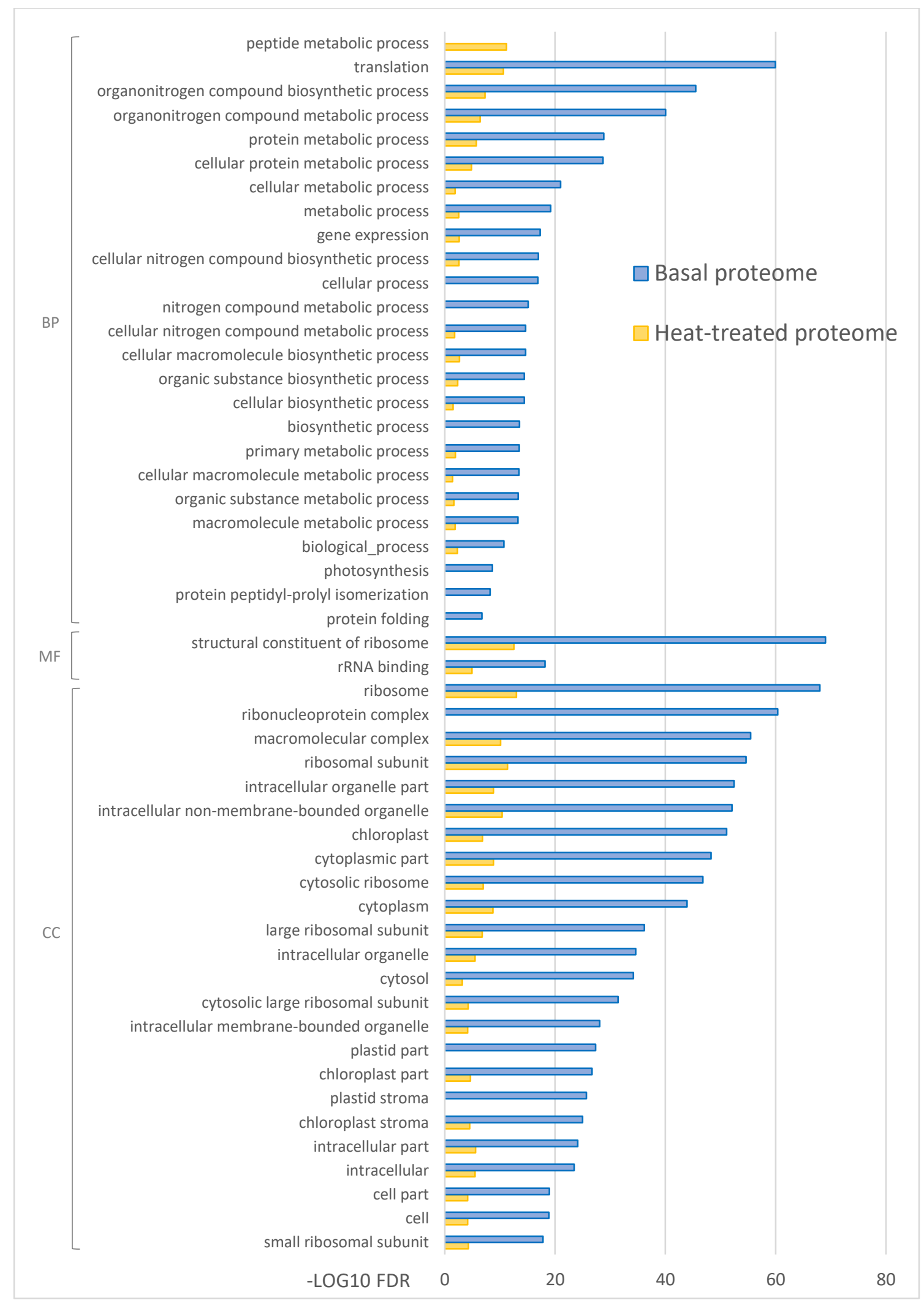

Figure 4.25. GO enrichment of proteins differentially expressed in amiR23.5/23.6/26.5 relative to control plants in normal and after heat treatment by using STRING. BP: biological process, MF: molecular function, CC: cellular compartment. Access 08.12.18. 


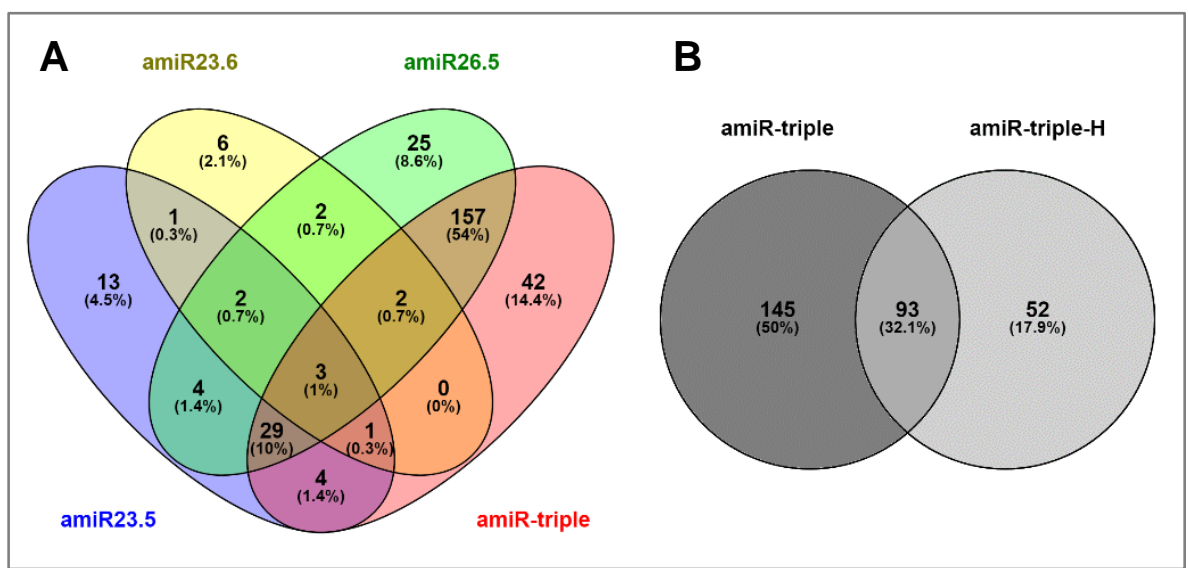

Figure 4.26. Venn diagrams showing numbers of differentially expressed proteins in amiR compare to control plants by using Venny2.1. (A) Number of commonly changed proteins in amiR23.5, amiR23.6, amiR26.5 and in the triple amiR23.5/23.6/26.5. (B) Number of significantly changed proteins in the normal proteome of triple amiR23.5/23.6/26.5 and in the heat-treated proteome.

\subsubsection{Reduction in the SHSP-M content leads to profound metabolic alterations.}

The differential proteomic analysis of the knockdown mutants and controls seedlings revealed significant upregulation of various metabolism-related proteins. In the double mutant amiR23.5/23.6, 128 proteins probably involved in metabolic pathways were differentially expressed. At least 140 metabolism-related proteins changed in the proteome of the triple mutant amiR23.5/23.6/26.5 compared to control proteome. In order to test whether the decreased abundance of SHSP-M lead to alteration in the primary metabolism of amiR mutants, metabolic profiling by GC-MC analysis was performed in Col-0 and mutant plants. Double amiR23.5/23.6 and triple amiR23.5/23.6/26.5 mutants were evaluated by this approach. Metabolite extraction of 15- and 28-day-old mutants and Col-0 plants grown under normal conditions were performed. Experiments using 15-day-old plants were based on three biological replicates corresponding to three independent lines of each mutant. Replicates consisted in a pool of 50 plants. In the experiment with 28-day-old plants, three independent lines of each mutant were evaluated, each of them with three biological replicates. In this case, replicates consisted in a pool of 3 plants. A total of 25 and 23 metabolites were identified and quantified in every chromatogram of 15- and 28-day-old plants, respectively. These included amino acids, organic and fatty acids, and sugars.

In 15-day-old plants, double amiR23.5/23.6 and triple mutants accumulated several amino acids (Table 4.14). Serine, methionine, glutamine, and proline showed higher levels in both mutants. It is worth mentioning that methionine accumulated to a 3.5-fold level in the double mutant amiR23.5/23.6 and to a 45-fold in the triple mutant plants. Besides these, asparagine and threonine accumulated in the double mutants and aspartic acid in the triple amiR mutant. Asparagine showed high levels in amiR-T but considerable variability in the results lead to no statistical significance. Among the pool of organic acids, both double and triple mutants 
showed increased levels of fumaric acid with more than 3.3-fold level in double mutant amiR23.5/23.6 and citrate which showed a significant accumulation of 13.2-fold change in the triple amiR-T. Maleic and phosphoric acids were differentially increased in the double mutant. Of note, amiR-triple lines showed a reduction of oxalic acid and stearic acid. Fructose and galactose highly accumulated in the triple amiR mutant while sucrose showed accumulation mainly in the double amiR mutant. Lyxose, on the other hand, significantly increased in both knockdown lines, reaching a 5.4-fold and 5.7-fold higher accumulation in amiR-double and amiR-triple, respectively. The levels of myo-inositol were also altered, this small molecule that is crucial in the regulation of growth and development [Donahue, 2010] increased in both mutants. Ascorbic acid showed significant accumulation (8.5- fold) in the triple amiR mutant.

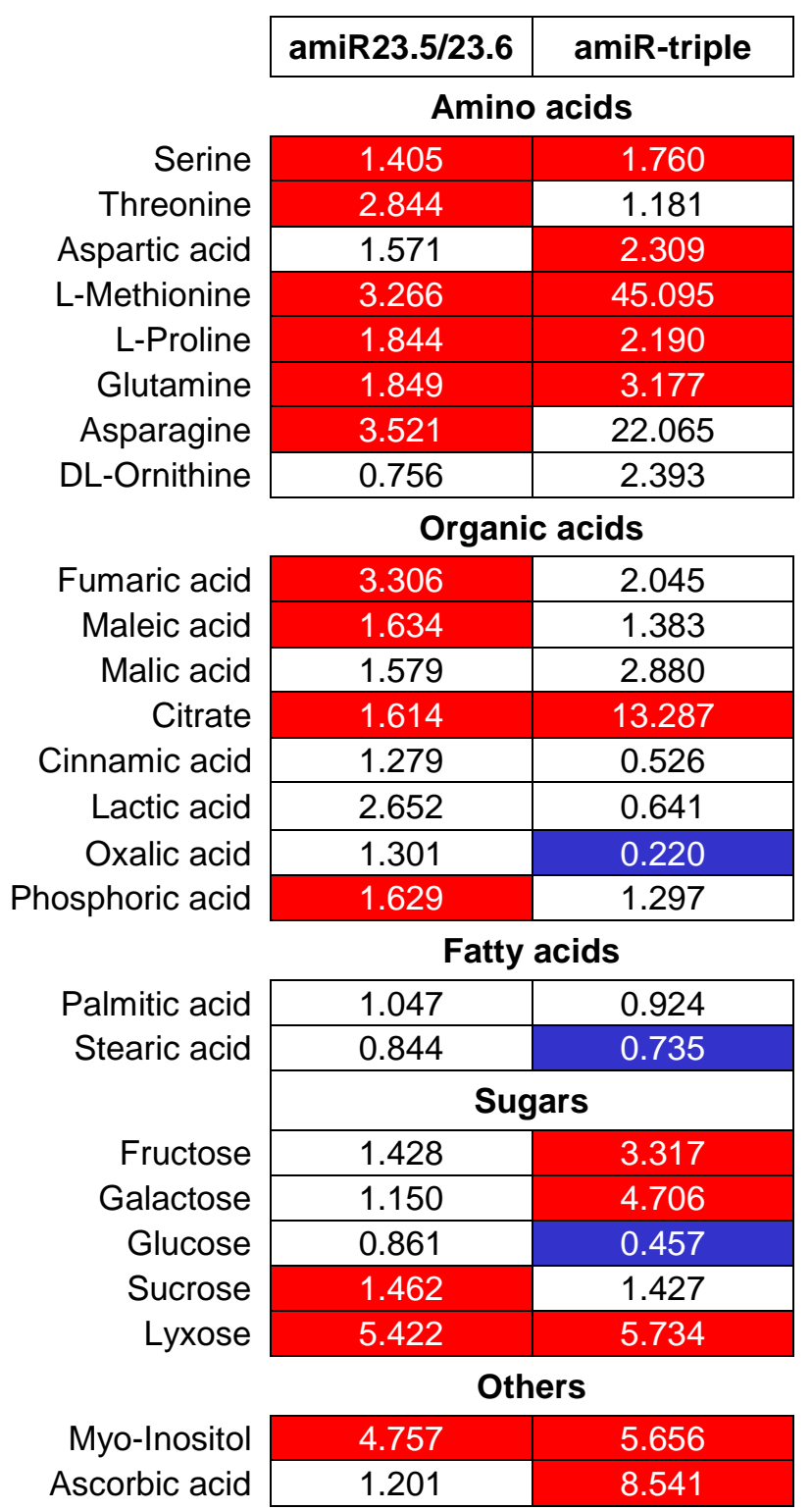

Table 4.14. Metabolic profiling of amiR23.5/23.6 and amiR23.5/23.6/26.5 by GC-MS. Metabolites were extracted from 15 day-old mutants and Col-0 plants. Numbers indicate fold changes relative to Col- 0 values. Significant up-regulated (red) and down-regulated (blue) metabolites are shown $(p=0.05$ by t-test analysis). White color was used to indicate no statistical significance. 
To gain more insight into the metabolic modifications observed in double amiR23.5/23.6 and triple mutants, 28 day-old plants were examined by GC-MS and a list of altered metabolites was obtained (Table 4.15). Most of the detected metabolites suffered a significant reduction in the triple mutant lines (blue color). Except for maleic acid which exhibited non-significant change, the rest of the organic acids decreased in these mutants. Down-regulation of amino acids, fatty acids, and sugars was observed for the amiR-T. In the mutant with double reduction of sHSP23.5 and SHSP23.6, the metabolic pattern was more variable. Among the detected amino acids, serine and threonine significantly accumulated whereas aspartic acid and proline decreased. From the organic acid group, succinic, fumaric, cinnamic, and glyceric acids showed significant accumulation in at least two independent lines. On the other side, citric, lactic and oxalic acids suffered a reduction in these lines. Both fatty acids, palmitic and stearic acid increased in the double amiR23.5/23.6 mutant as well as the myo-inositol and glycerol. Sugars presented distinct patterns: some like sucrose and lyxose accumulated and at the same time, others like fructose, galactose, and glucose, decreased.

\begin{tabular}{|c|c|c|c|c|c|c|}
\hline & $\begin{array}{c}\text { amiR- } \\
\text { double-1 }\end{array}$ & $\begin{array}{c}\text { amiR- } \\
\text { double-2 }\end{array}$ & $\begin{array}{c}\text { amiR- } \\
\text { double-3 }\end{array}$ & $\begin{array}{c}\text { amiR- } \\
\text { triple-1 }\end{array}$ & $\begin{array}{c}\text { amiR- } \\
\text { triple-2 }\end{array}$ & $\begin{array}{c}\text { amiR- } \\
\text { triple-3 }\end{array}$ \\
\hline & \multicolumn{6}{|c|}{ Amino acids } \\
\hline Serine & 5.589 & 5.944 & 4.167 & 0.276 & 0.291 & 0.338 \\
\hline Threonine & 6.507 & 3.659 & 2.072 & 0.134 & 0.233 & 0.124 \\
\hline Aspartic acid & 0.037 & 0.066 & 0.038 & 0.134 & 0.120 & 0.120 \\
\hline \multirow[t]{2}{*}{ Proline } & 0.602 & 0.696 & 0.361 & 0.325 & 0.288 & 0.422 \\
\hline & \multicolumn{6}{|c|}{ Organic acids } \\
\hline Succinic acid & 1.096 & 1.760 & 2.231 & 0.168 & 0.118 & 0.149 \\
\hline Fumaric acid & 2.784 & 4.446 & 4.577 & 0.079 & 0.105 & 0.147 \\
\hline Maleic acid & 0.258 & 0.411 & 0.244 & 1.082 & 0.605 & 0.672 \\
\hline Malic acid & 0.944 & 0.844 & 0.906 & 0.297 & 0.230 & 0.230 \\
\hline Citrate & 0.545 & 0.747 & 0.486 & 0.335 & 0.157 & 0.245 \\
\hline Cinnamic acid & 1.188 & 1.811 & 1.511 & 0.748 & 0.489 & 0.606 \\
\hline Lactic acid & 0.691 & 0.277 & 0.436 & 0.311 & 0.362 & 0.330 \\
\hline Oxalic acid & 0.493 & 0.595 & 0.412 & 0.303 & 0.290 & 0.272 \\
\hline Phosphoric acid & 0.807 & 1.195 & 1.056 & 0.248 & 0.220 & 0.258 \\
\hline \multirow[t]{2}{*}{ Glyceric acid } & 1.616 & 2.330 & 2.559 & 0.222 & 0.149 & 0.167 \\
\hline & \multicolumn{6}{|c|}{ Fatty acids } \\
\hline Palmitic acid & 1.955 & 2.184 & 2.779 & 0.343 & 0.536 & 0.509 \\
\hline \multirow[t]{2}{*}{ Stearic acid } & 2.001 & 2.463 & 3.471 & 0.398 & 0.529 & 0.602 \\
\hline & \multicolumn{6}{|c|}{ Sugars } \\
\hline Fructose & 0.417 & 0.638 & 0.523 & 0.223 & 0.165 & 0.179 \\
\hline Galactose & 0.521 & 0.568 & 0.871 & 0.430 & 0.174 & 0.286 \\
\hline Glucose & 0.441 & 0.694 & 0.811 & 0.188 & 0.167 & 0.184 \\
\hline Sucrose & 1.756 & 3.759 & 2.789 & 0.388 & 0.239 & 0.254 \\
\hline \multirow[t]{2}{*}{ Lyxose } & 1.889 & 3.075 & 2.420 & 0.622 & 0.513 & 0.824 \\
\hline & \multicolumn{6}{|c|}{ Others } \\
\hline Myo-Inositol & 1.412 & 1.936 & 1.803 & 0.380 & 0.284 & 0.353 \\
\hline Glycerol & 3.215 & 4.388 & 2.106 & 0.268 & 0.230 & 0.220 \\
\hline
\end{tabular}


Table 4.15. Metabolic profiling of amiR23.5/23.6 and amiR23.5/23.6/26.5 by GC-MS. Metabolites were extracted from 28 day-old mutants and Col-0 plants. Numbers indicate fold changes relative to Col-0 values. Significant up-regulated (red) and down-regulated (blue) metabolites are shown ( $p=0.05$ by t-test analysis). White color was used to indicate no statistical significance.

To further confirm the reduction of proline observed in the 28-days triple mutants, free proline content was also determined by spectrophotometry (Fig. 4.27). The amiR-triple mutant had significantly lower levels of free proline in the three independent lines that were analyzed compared to Col-0 plants.

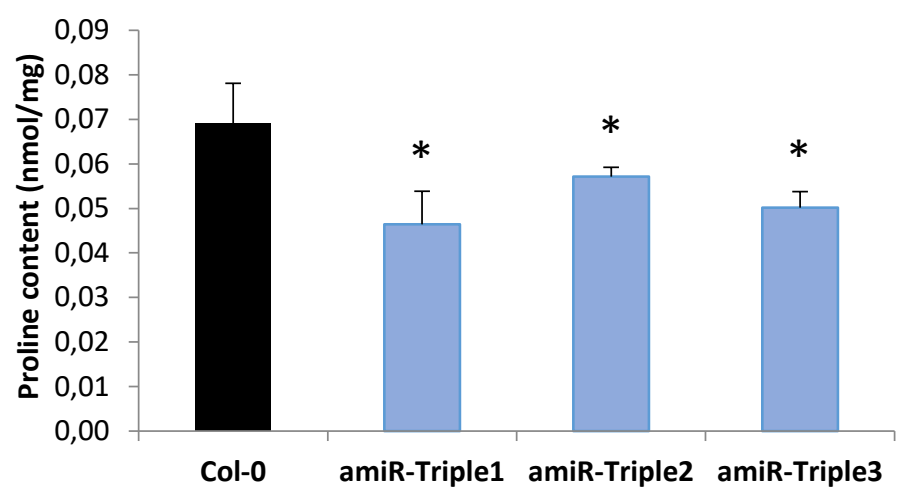

Figure 4.27. Free Proline content in amiR-triple and Col-0 plants. Proline was extracted from adult 28 day-old plants grown under normal conditions. Three independent transgenic lines were measured. Each data point corresponds to the mean values $+S D$ of four biological replicates. Each replicate consisted of a pool of 3 plants. Asterisks represent significance by one-sided t-test with $p<0.05$.

\subsubsection{5. amiR-triple mutant exhibited up-regulation of several photosynthetic related proteins.}

As it was previously described, triple amiR mutant plants produce small and chlorotic leaves. Figure 4.28-A. shows 28 day-old mutants and Col-0 plants. It can be seen the pale green leaves in the triple mutants and the veins which are especially evident in these plants. Furthermore, several proteins which are related to photosynthesis differentially accumulated in the proteome of amiR-triple mutant (Table 4.16). In order to test if the downregulation of sHSP-M produced an alteration of the photosynthetic processes, chlorophylls, and other pigments were determined. The small measurable area in amiR-triple leaves made difficult the evaluation of the activity of photosystem I and II and of other important photosynthetic parameters. For pigments determination, samples were taken from 28 day-old plants grown under normal conditions. Four independent triple knockdown lines were evaluated and 8 plants were measured in each line. Among the quantified pigments, the levels of chlorophyll a showed a significant reduction in all the triple mutant lines (Fig. 4.28-B). Chlorophyll $b$ and carotenoids and xanthophylls exhibited reduced levels in only one triple mutant line. 


\begin{tabular}{lllll}
\hline Accession & $\begin{array}{c}\text { Uniprot } \\
\text { code }\end{array}$ & \multicolumn{1}{c}{ Description } & $\begin{array}{c}\text { Fold change } \\
\text { (log })\end{array}$ & Adj. P-Value \\
\hline AT1G77090 & O49292 & $\begin{array}{l}\text { Mog1/PsbP/DUF1795-like photosystem II } \\
\text { reaction center PsbP family protein }\end{array}$ & 6.64 & $6.70 \mathrm{E}-17$ \\
\hline AT4G03280 & Q9ZR03 & photosynthetic electron transfer C & 6.64 & $6.70 \mathrm{E}-17$ \\
\hline AT3G60370 & Q0WRJ7 & $\begin{array}{l}\text { FKBP-like peptidyl-prolyl cis-trans isomerase } \\
\text { family protein }\end{array}$ & 6.64 & $6.70 \mathrm{E}-17$ \\
\hline AT4G28750 & Q9S831 & $\begin{array}{l}\text { Photosystem I reaction center subunit IV / } \\
\text { PsaE protein }\end{array}$ & 6.64 & $6.70 \mathrm{E}-17$ \\
\hline AT1G76450 & Q9S720 & $\begin{array}{l}\text { Photosystem II reaction center PsbP family } \\
\text { protein }\end{array}$ & 6.64 & $6.70 \mathrm{E}-17$ \\
\hline AT2G20260 & Q9S714 & photosystem I subunit E-2 & 6.64 & $6.70 \mathrm{E}-17$ \\
\hline AT5G38430 & P10796 & $\begin{array}{l}\text { Ribulose bisphosphate carboxylase (small } \\
\text { chain) family protein }\end{array}$ & 5.61 & $6.70 \mathrm{E}-17$ \\
\hline AT4G02770 & Q9S7H1 & photosystem I subunit D-1 & 4.35 & $6.70 \mathrm{E}-17$ \\
\hline AT5G38410 & B3H5S2 & $\begin{array}{l}\text { Ribulose bisphosphate carboxylase (small } \\
\text { chain) family protein }\end{array}$ & 4.1 & $6.70 \mathrm{E}-17$ \\
\hline AT4G12800 & Q9SU14 & photosystem I subunit I & 3.74 & $6.70 \mathrm{E}-17$ \\
\hline AT1G06680 & Q42029 & photosystem II subunit P-1 & 3.64 & $2.94 \mathrm{E}-15$ \\
\hline AT1G67090 & F4HRR5 & $\begin{array}{l}\text { ribulose bisphosphate carboxylase small } \\
\text { chain 1A }\end{array}$ & 3.52 & $2.60 \mathrm{E}-14$ \\
\hline AT4G21280 & Q9XFT3 & photosystem II subunit QA & 2.91 & $4.96 \mathrm{E}-10$ \\
\hline AT4G28660 & F4JM05 & photosystem II reaction center PSB28 protein & 2.89 & $1.06 \mathrm{E}-08$ \\
\hline AT1G54780 & Q9ZVL6 & thylakoid lumen 18.3 kDa protein & 2.62 & $2.78 \mathrm{E}-08$ \\
\hline AT3G55330 & P82538 & PsbP-like protein 1 & 2.39 & $5.76 \mathrm{E}-07$ \\
\hline
\end{tabular}

Table 4.16. List of photosynthesis-related proteins in the amiR-triple mutant showing significant level changes relative to control plants.

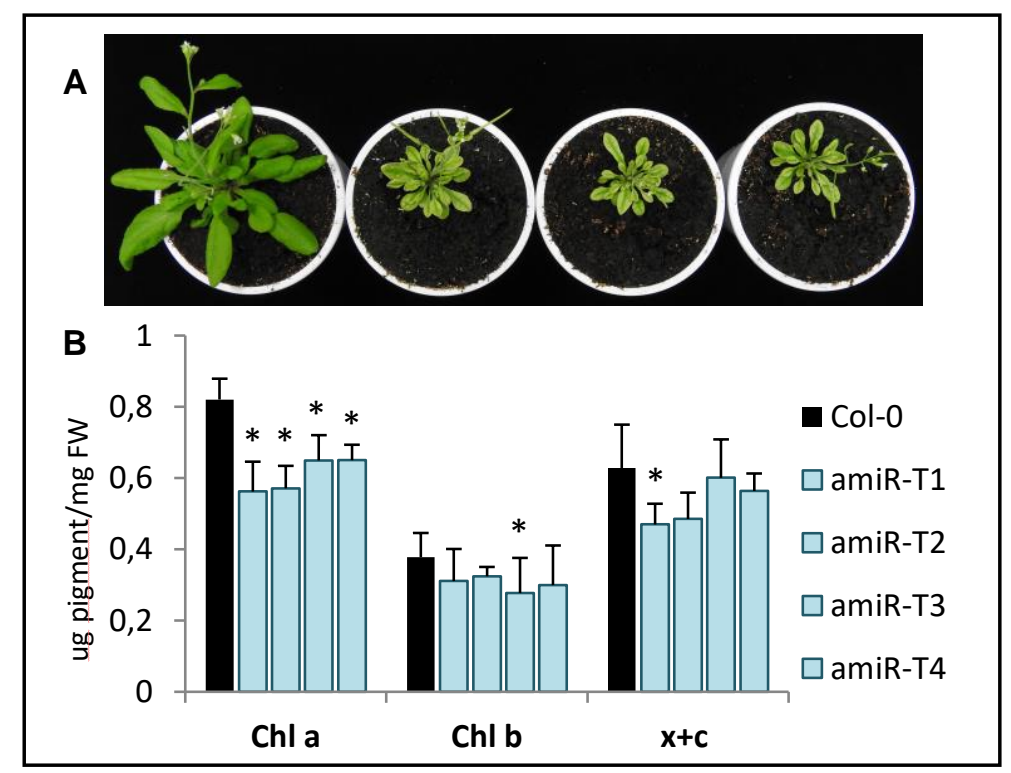

Figure 4.28. A) Chlorotic and dwarf phenotype of triple amiR mutants compared to Col-0 (the first on the left). B) Pigments content in amiR-triples and Col-0 plants. Chlorophyll $a$, chlorophyll $b$, and xanthophylls and carotenoids $(x+c)$ were extracted from adult 28 day-old plants. Four independent transgenic lines were measured. The data points in the graph correspond to the mean value $+S D$ of 8 biological replicates. Asterisks mean statistical significance by one-sided t-test with $p<0.05$. 


\subsubsection{Up-regulation of ROS detoxifying enzymes in the amiR mutants.}

Proteomic data revealed several enriched GO terms and proteins that are related to oxidative stress and to the detoxification of reactive oxygen species (ROS) in the mutants. As it was presented in Fig. 4.18-B, Panther analysis at the $1^{\text {st }}$ level of ontology showed specific overrepresentation of antioxidant activity in the single mutant amiR26.5 unlike the other single amiR23.5 and amiR23.6 mutants. Nine proteins with essential roles in ROS detoxification were upregulated in the amiR26.5 mutant when compared to control plants (Table 4.17). Among them, the peroxiredoxin IIF (PRXIIF), glutathione peroxidase 6 (GPX6), and 2-cysteine peroxiredoxin $B(P R X I I B)$ were found. Some proteins related to oxidative stress were also increased in the proteome of double amiR and triple amiR mutants (Suppl. Table 4.4-4.5). In the triple amiR, four protein were increased including three peroxidases (PRXIIF, PRXIIB, PRXIIE) (highlighted in Suppl. Tables 4.5). This increased antioxidant capacity in the amiR26.5, amiR23.5/23.6, and triple mutants should be reflected in alleviated levels of ROS. To prove this hypothesis, the production of reactive oxygen species in adult plants was determined according to standard histochemical detection. The level of $\mathrm{O}_{2} \cdot-$ and $\mathrm{H}_{2} \mathrm{O}_{2}$ in leaves of 28 day-old plants were evaluated in Col-0 and mutant plants. Accumulation of $\mathrm{H}_{2} \mathrm{O}_{2}$ was found in leaves of single amiR23.5 and amiR23.6, and in the double amiR23.5/23.6 as it can be concluded from the dark brown color in these mutants (Fig. 4.29-A). On the other side, a reduced amount of the dark brown color was observed in the single amiR26.5 and in the amiRtriples mutants. Leaves exhibited a pain color staining what indicates less amount of $\mathrm{H}_{2} \mathrm{O}_{2}$. The results of NBT staining assays revealed a slight $\mathrm{O}_{2}$ - increase in amiR23.6 and in the double mutant amiR23.5/23.6 while leaves from amiR23.5 lines appeared to have control-like $\mathrm{O}_{2}$-levels (Fig. 4.29-B). Triple mutant lines seem to strongly accumulate superoxide anion leading to a deep blue coloration. amiR26.5 showed again no accumulation of the $\mathrm{O}_{2}-$ molecule. Together, these histochemical and proteomic data indicate that amiR26.5 mutant may have increased capacity to decompose ROS eventually leading to better tolerance to oxidative stress conditions.

\begin{tabular}{lllll}
\hline Accession & $\begin{array}{c}\text { Uniprot } \\
\text { code }\end{array}$ & \multicolumn{1}{c}{ Description } & $\begin{array}{c}\text { Fold change } \\
\left(\mathbf{l o g}_{2}\right)\end{array}$ & Adj. P-Value \\
\hline AT3G06050 & Q9M7T0 & peroxiredoxin IIF(PRXIIF) & 6.64 & $6.29 \mathrm{E}-17$ \\
\hline AT1G65980 & Q9XEX2 & thioredoxin-dependent peroxidase 1 & 6.64 & $6.29 \mathrm{E}-17$ \\
\hline AT4G03520 & Q9SEU8 & thioredoxin superfamily protein & 6.64 & $6.29 \mathrm{E}-17$ \\
\hline AT4G11600 & O48646 & glutathione peroxidase 6 (GPX6) & 6.64 & $6.29 \mathrm{E}-17$ \\
\hline AT3G15360 & Q9SEU6 & thioredoxin M-type 4 & 4.87 & $6.29 \mathrm{E}-17$ \\
\hline AT5G06290 & Q9C5R8 & 2-cysteine peroxiredoxin B (PRXIIB) & 2.93 & $6.29 \mathrm{E}-17$ \\
\hline AT3G52960 & Q949U7 & thioredoxin superfamily protein (PRXIIE) & 1.67 & $1.26 \mathrm{E}-08$ \\
\hline AT3G11630 & Q96291 & thioredoxin superfamily protein & 1.58 & $2.50 \mathrm{E}-06$ \\
\hline AT3G26060 & Q9LU86 & thioredoxin superfamily protein & 1.42 & $1.34 \mathrm{E}-05$ \\
\hline
\end{tabular}

Table 4.17. List of ROS-related proteins in the ami26.5 mutant showing significant level changes relative to control plants. 
A

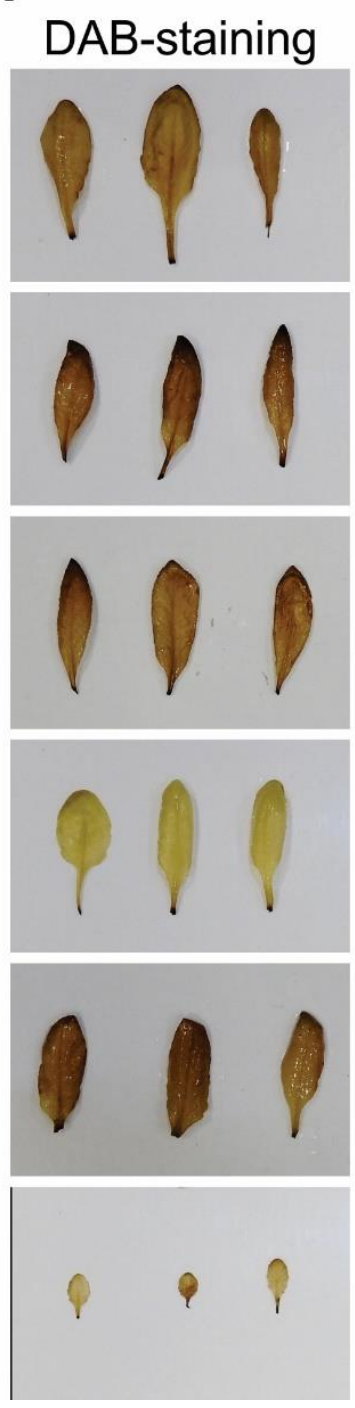

B

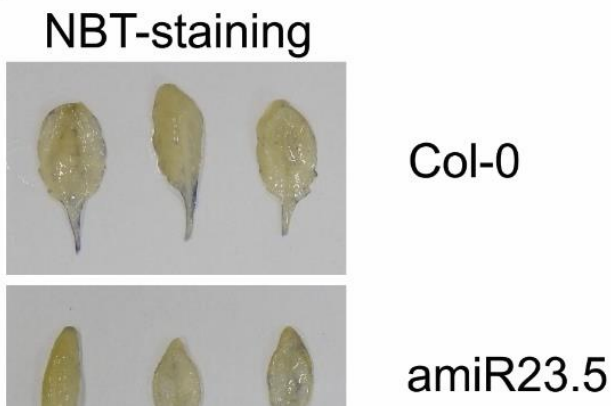

amiR23.5

amiR23.6
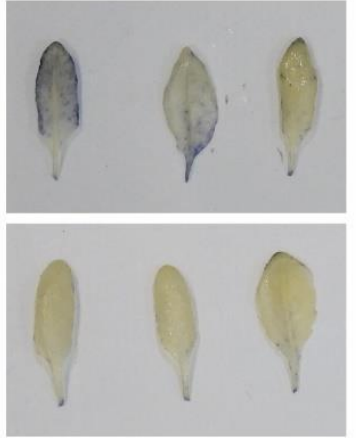

amiR26.5

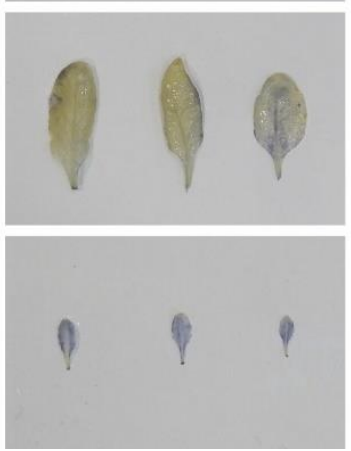

amiR23.5/23.6

amiR23.5/23.6/26.5

Figure 4.29. Histochemical detection of reactive oxygen species in amiR knockdown plants. Detection of $\mathrm{H}_{2} \mathrm{O}_{2}$ by DAB-staining $(A)$ and $\mathrm{O}_{2}-$ by NBT staining (B) was performed in leaves from 28 day-old plants.

\subsubsection{Cell membranes are highly affected in the knockdown sHSP-M mutants.}

High levels of ROS can be extremely harmful to cells by causing lipid peroxidation in cellular membranes, protein and carbohydrate oxidation, DNA damage and cell death [Gill, 2010]. The ion permeability of cell membranes, which is considered an indicator of cell damage induced by ROS was evaluated in the knockdown mutant plants. Mutants and Col-0 plants were grown under normal conditions and 28 day-old plants were subjected for $3 \mathrm{~h}$ to $37 \stackrel{\circ}{\mathrm{C}}$ followed by 1 $\mathrm{h}$ of recovery in the climate chamber. Leaf disks were cut from these plants before the treatment and directly after the recovery period and used to determine the electrolyte leakage. Single, double and triple amiR mutant plants showed higher electrolyte leakage compare to Col-0 plants as it is presented in Fig. 4.30-A. This higher amount of electrolytes in solution may be possible due to the loss of cell membrane stability and integrity. It is known that high and 
low temperature altered cell membrane ion permeability of plants leading to considerable loss of electrolyte. Figure 4.30-B shows the increase in the electrolyte leakage especially in amiR23.5 and amiR23.6 heat-treated mutants. Accumulation of electrolytes leaked was also observed in triple mutants while in amiR26.5 only one independent line showed a similar increase. Double amiR mutant showed no significant changes although a tendency to higher levels of electrolyte leaked can be seen.

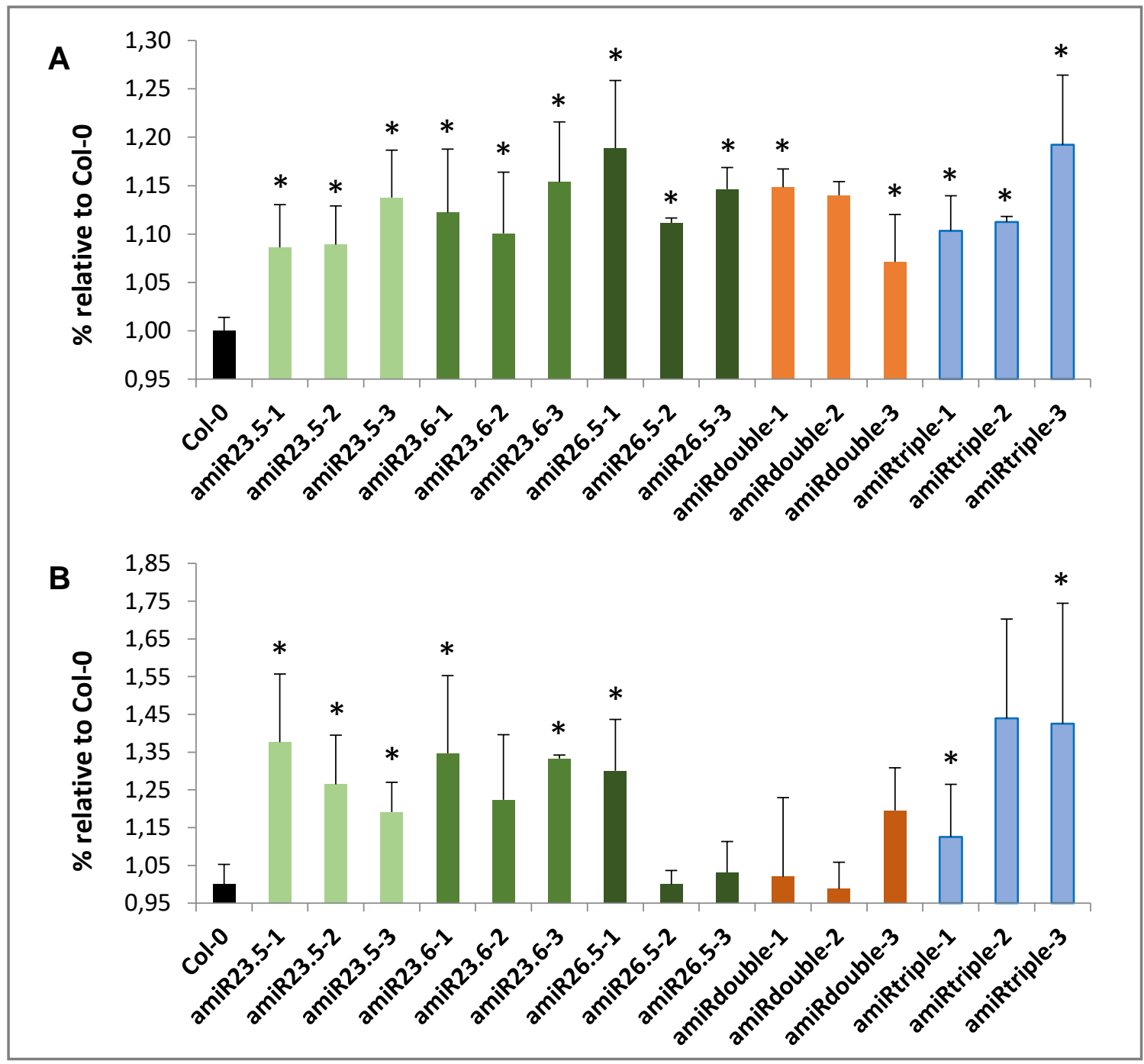

Figure 4.30. Electrolyte leakage in amiR knockdown and Col-0 plants. Determinations were performed on 28 day-old plants before (A) and after being exposed for $3 \mathrm{~h}$ at $37^{\circ} \mathrm{C}$ followed by $1 \mathrm{~h}$ of recovering at normal temperature (B). Three independent transgenic lines were measured for each mutant. Results are presented as relative to Col-0 values. Data points represent the mean value + SD of three biological replicates. Asterisks mean significance by one-sided t-test with $p<0.05$.

The high loss of membrane integrity may cause severe damage and cell death. To visualize cell death in amiR mutant lines trypan blue staining was performed. Mutants and Col-0 plants were grown under normal conditions and leaf number 8 was cut out from 28-day-old plants 
and stained with tryphan blue. While in single and double mutants tryphan blue staining did not show accumulation, the staining was significantly higher in the triple amiR. It can be appreciated in Fig. 4.31, the deep blue color in the leaves of the triple mutant plants.

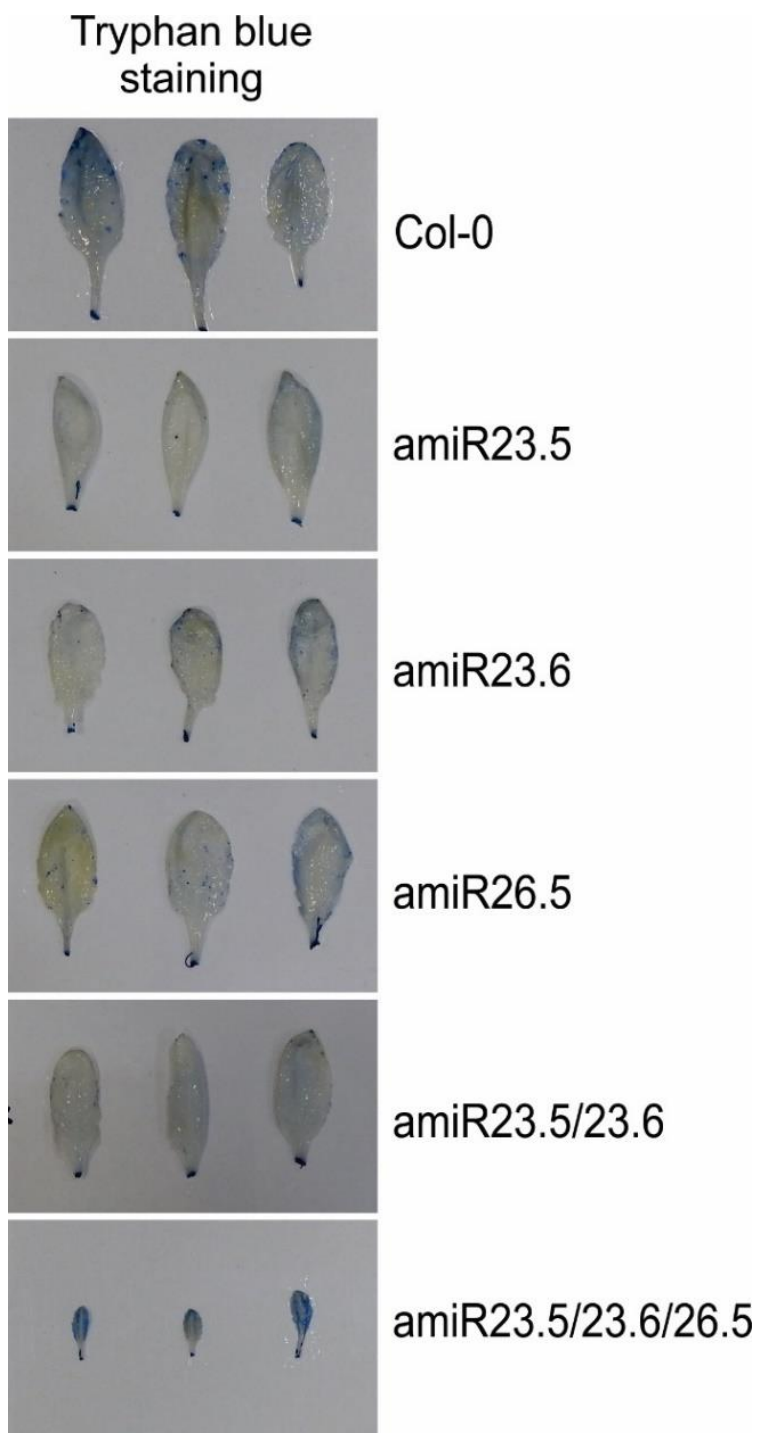

Figure 4.31. Cell death visualization with trypan blue staining in amiR mutant plants. Staining was performed on leaves from 28 day-old plants grown under normal conditions. Note the strong staining of the triple amiR23.5/23.6/26.5 leaves. 


\subsubsection{Discussion}

The function of organellar sHSPs in plants under stress conditions and during normal development is to date largely unknown. In this work, functional characterization of the mitochondrial small heat shock proteins under stress conditions and during $A$. thaliana development was presented.

Firstly, paralogous of the sHSPs-M were investigated to discover other proteins that were closely related and could function as mitochondrial SHSP. Apart from the already mentioned sHSPs-M, only one additional protein appeared, the HSP21 located in chloroplasts (Table 4.10). Sequence comparison between these proteins revealed that sHSP23.5 and SHSP23.6 share high similarity in their sequences (around 70\%) (Table 4.11). Far from this, sHSP26.5 which is also 22 amino acids longer, show no more than $35 \%$ of sequence similarity compared to the others SHSP-M. Subcellular localization of these three proteins to mitochondria has been predicted and confirmed in previews studies [Siddique, 2008; Van Aken, 2009]. Mitochondria perform many essential functions in the cell and thus disruption or alterations in their metabolic activities may affect the cell viability. SHSPs-M were formerly associated with the protection of mitochondrial proteins and with the thermotolerance of mitochondria [Chou, 1989; Sanmiya, 1989]. Considering these findings and the fact that three sHSP are located in mitochondria in A. thaliana, it can be speculated that these proteins might play crucial roles in the cell homeostasis.

Analysis of the sHSPs-M expression by q-PCR under normal conditions clearly showed the low basal expression of these genes. On the other hand, the three sHSPs-M were strongly induced by the heat shock treatment with more than 400 -fold enrichment. sHSP26.5 was also up-regulated by cold $\left(4^{\circ} \mathrm{C}\right)$ and $s H S P 23.5$ down-regulated by salinity. Similar up-regulation by high temperatures has been previously reported for sHSP23.5 and sHSP23.6 [Waters, 2008a]. Promoters activities corroborated up-regulation of the three $s H S P-M$ upon heat shock, but sHSP23.5 promoter was responsive to other stresses (Fig. 4.9). Notably, the promoter activity of sHSP26.5 appeared to be restricted to roots while the other two promoters exhibited increased activity in all tissues. Once again, sHSP26.5 differentiates from the sHSP23.5 and sHSP23.6. Moreover, various cis-regulatory motifs belonging to the HEAT family were found in the three promoter sequences, although in a different location, in sHSP23.5 promoter three HEAT elements are in the proximal region of transcription initiation while in sHSP23.6 and sHSP26.5 promoters, most HEAT elements are in a distal region (Fig. 4.10). All these data indicate that mitochondrial sHSPs may be mainly regulated by high temperature, probably at a different level.

To further explore the function of SHSPs-M single, double and triple knockdown mutants (amiR23.5/23.6/26.5) of $A$. thaliana were examined. Numerous transgenic lines were successfully generated and evaluated at the phenotypic, molecular and biochemical level. 
Single knockdown mutants of sHSP23.5 and sHSP23.6 displayed similar phenotype. They grew normally but produced rosettes of bigger sizes compare to Col-0 plants. Apart from being bigger, leaves of these mutants appeared to be curved indicating an alteration during leaf development. Knockdown mutants of sHSP26.5 did not show any of these characteristics. These mutants exhibited normal growth and do not seem to have compromised their vegetative and reproductive development showing a Col-0-like phenotype. Nevertheless, when root growth was evaluated the only single amiR mutant that displayed alterations in this tissue was amiR26.5: their roots were significantly shorter than Col-0 plants. Roots of single mutants amiR23.5 and amiR23.6 did not differentiate from Col-0 roots. This is in concordance with the pattern of expression of the genes as it can be speculated from the promoter activity analysis. The promoter of SHSP23.5 and sHSP23.6 was stronger in the aerial parts of seedlings while the promoter of SHSP26.5 showed higher activity only in roots. Thus, single mutants amiR23.5 and amiR23.6 were mainly affected in leaves while single mutant amiR23.6 showed alterations in roots. The phenotype observed in single mutants amiR23.5 and amiR23.6 was also found in mutants where both genes were simultaneously down-regulated. Double mutant amiR23.5/23.6 produced rosettes with bigger leaves than Col-0 plants. Beside this, leaves showed the same curvatures as those found in the single mutants. However, double mutants amiR23.5/23.6 do not exhibit an exacerbated phenotype compared to the singles amiR mutants. These may indicate a probable redundancy of function and compensation given by the third sHSP26.5. The triple knockdown amiR mutant displayed, unlike double and single mutants, a strongly affected phenotype. Plants were considerably smaller indicating a profound alteration in the plant development. A comparison between leaves from triple amiR mutant and Col-0 plants showed a clear difference in size, with more than 4-fold smaller leaves in the triple amiR mutant. Epidermal cells areas were evaluated in order to investigate the possible reasons for having small leaves. Epidermal cells of amiR-T plants were significantly smaller than Col-0 whereas no differences in the cell number were found. Considering the results obtained from the cell areas observation, it can be concluded that the small size of amiR-T plants is mainly due to the alteration in the process of cell expansion but not in the cellular proliferation. Apart from being small, leaves of amiR-T were narrower, chlorotic and showed a mildly reticulated phenotype. These mutants exhibited lower levels of chlorophyll $a$ and up-regulation of several photosynthesis-related proteins in the amiR-T proteome. Not only leaves were smaller but also whole plants showed smaller size compared to Col-0 plants. Rosettes were tiny as a consequence of the leaf size and stems were shorter resulting in dwarf plants. Additionally, these mutants showed decreased seed production with 12-fold lower seed yield than control plants. Like amiR26.5, triple amiR produced significantly shorter roots that reached no more than $2.5 \mathrm{~cm}$ after 15 days of growth. This altered phenotype can be explained by the expression pattern of the sHSPs-M, sHSP23.5 and sHSP23.5 are mostly expressed in leaves 
and SHSP26.5 in roots. Moreover, in single and double mutants, functional redundancy may probably compensate for the effects of the genes down-regulation. When the three $S H S P-M$ are knocked down functional compensation is no longer possible and the consequences in the phenotype became evident as seen in the triple amiR mutant.

OMICS technologies such as proteomics and metabolomics are highly useful techniques to elucidate and explore changes occurring at a more global scale [Feussner, 2015]. These techniques provide a huge amount of data about metabolites and proteins being modified and thus can be extremely informative in the study of sHSP-M functions. The present work provides information on the differential protein and metabolites abundance between knockdown mutants and Col-0 plants. Down-regulation of the mitochondrial SHSPs produced profound proteome-wide changes as it can be assumed from the high number of proteins that significantly changed. Interestingly, most of the proteome accumulated to higher levels and only a few proteins decrease in the mutants. In seedlings grown under normal conditions, amiR26.5, amiR23.5/23.6, and amiR23.5/23.6/26.5 showed differential accumulation of more than 200 proteins compared to control plants. The number of changing proteins was considerably smaller in the amiR23.5 and amiR23.6. This indicates that, although no major changes were observed in the phenotype of amiR26.5, reduced level of sHSP26.5 produced wide effects on protein homeostasis. Similarly, simultaneously reduction of two and the three mitochondrial sHSPs lead to important changes in the basal proteome and consequently in the phenotype. On the other hand, individual reduction of sHSP23.5 and sHSP23.6 appeared to produce less severe effects in the proteome of the mutants. However, when compared the differential basal proteome with the differential proteome of heat-treated mutants, the number of proteins with modified abundances increased after heat only in the amiR23.5 and amiR23.6. In the rest of the analyzed mutants, a smaller number of differential proteins were observed in the treated compared to non-treated plants. These results imply that proteomes of single mutants amiR23.5 and amiR23.6 are more vulnerable and responsive to the heat shock stress, not like the other mutants. Proteomic analysis of the single amiR showed similarities in the gene ontology annotation of the changed proteins at the first level of ontology of Panther and by using String. It can be speculated though, from the low number of common changed proteins between single amiRs, that low abundance of the individual sHSP-M produced distinct proteomes response.

All amiR mutants displayed alterations in the abundance of several proteins related to translation and to the ribosome functioning and structure. Triple amiR mutant exhibited a higher number of proteins with differential abundance involved in these processes compared to the other single and double amiR23.5/23.6 mutants. Such a wide change in ribosome-related proteins may indicate a possible alteration in the proper ribosome function. It has been reported that dysfunction of ribosomes can affect the translation of certain transcripts that are important 
in the leaf development [Horiguchi, 2011]. Several studies have shown that mutants deficient in different ribosome proteins have abnormal phenotypes suggesting that ribosomes have specialized developmental functions in addition to their role in translation. Interestingly, different aspects of the triple amiR phenotype were also found in these r-proteins mutants. For example, a mutation in the cytoplasmic ribosomal protein RPS13A produced narrow pointed leaves and inhibition of root growth like in the amiR-T mutant [Ito, 2000]. The mutation also affects the cell division activity leading to small leaves. Mutants of the ribosomal proteins RPL7B and RPS6A produce chlorotic and reticulated leaves, similar to amiR-T leaves. Other r-proteins mutants that were characterized displayed also comparable phenotype in leaves [Horiguchi, 2011; Carroll, 2013]. In some of them, reductions in both cell division and cell expansion contributed to the small leaf size while others were mainly affected by only one of these two processes [Horiguchi, 2011]. In this work, amiR-T displayed small narrow leaves resembling the phenotype of $r$-proteins mutants. Even though the leaf cell number in this mutant was not altered, cell areas were considerably smaller indicating that the cell elongation process is controlling the leaf size. It can be hypothesized that the alteration of several ribosome-related proteins due to the down-regulation of the three $s H S P s-M$, lead to a partial or complete dysfunction of ribosomes and the perturbation in the leaf development.

Apart from the correct function of ribosomes, plant development requires the optimal functioning of mitochondria and chloroplasts. Disruption in the mitochondrial function, for instance, can result in serious changes in the energy metabolism. Proteome analysis revealed the accumulation of a high number of proteins involved in the primary metabolism and several biosynthetic processes. Analysis of the metabolome of double and triple amiR mutants by GCMS showed altered levels of numerous metabolites including amino acids, organic and fatty acids, and sugars. Nevertheless, the metabolic state of 15 day-old amiR mutants differed from the one found in 28 day-old plants. In 15 day-old plants, most of the detected metabolites accumulated in both double and triple mutants. amiR plants showed enrichment of several amino acids such as serine, glutamine methionine, and proline. Of special interest is the accumulation of proline which has important functions in energy utilization, reactive oxygen species (ROS) generation, development and stress resistance. Proline metabolism includes the interconversion of proline and glutamate thanks to two mitochondrial enzymes, in a process that affects cellular energetics through the respiratory electron transport chain [Zhang, 2015]. There was also increased in some TCA cycle intermediates including fumaric acid and citrate, and in several sugars. This accumulation of organic acids and amino acids implicates an alteration at the central metabolism level. Accumulation of metabolites was previously reported in mutants with loss of mitochondrial function [Meyer, 2009; Tomaz, 2010]. Mutants defective in prohibitin 3, a protein of the inner mitochondrial membrane and in the organellar RNA polymerase important for the mitochondrial activity, showed the same accumulation of 
metabolites [Van Aken, 2016]. These mutants with mitochondrial defect showed reduction in the rosette size, growth retardation, lower seed production, and shorter roots. Although double amiR23.5/23.6 mutant did not exhibit these alterations, triple amiR mutants phenocopied almost all of them. The accumulation of several intermediates suggests that photosynthesis may be functioning properly in these mutants producing enough substrates for glycolysis. On the other hand, limited and slow processing of these metabolites into growth-supporting compounds may lead to their accumulation, as it was observed. Metabolites profiles were nearly the opposite in the 28 day-old triple amiR mutant. While at this age double amiR mutants showed accumulation of certain metabolites and reduction of others, triple amiR suffered a significant reduction of all of them. The depletion of metabolites in the triple amiR mutant suggests a constriction of primary metabolism and profound mitochondrial dysfunction. In concordance with this, it has been proved that the inhibition of complex I in $A$. thaliana by rotenone induced a significant alteration in the mitochondria function, reduced cell respiration and the depletion of several metabolites [Garmier, 2008]. Along with the reduction of several sugars, amino and organic acids, the molecule myo-Inositol showed significantly lower levels in the amiR-T lines. Since an oxidized form of inositol is the most common and important sugar involved in the production of cell walls polysaccharide, this molecule is essential in the cell wall biosynthesis [Loewus, 2000]. Two fatty acids, palmitic and stearic were also reduced in the triple amiR mutant. These fatty acids are found in almost all lipids classes of cell membranes. This may suggest that the overall membrane fluidity is changed in the triple mutant.

The proteomic approach used in this work also revealed the up-regulation of several proteins involved in redox processes and ROS response. In particular, the differential proteome of amiR26.5 analyzed by Panther showed an overrepresentation of the antioxidant activity process. In addition to this, histochemical staining showed that leaves from amiR26.5 lines did not accumulate $\mathrm{O}_{2} \cdot-$ and $\mathrm{H}_{2} \mathrm{O}_{2}$. This may indicate that, compared to Col-0 plants, amiR26.5 may better tolerate and mitigate the effects of ROS leading to a lower amount of these reactive molecules. Additional experiments should be performed in order to confirm this hypothesis. The abundance of several peroxidases in the triple amiR as it was found in the proteomic analysis, might be responsible for the reduction in $\mathrm{H}_{2} \mathrm{O}_{2}$ levels, albeit accumulation of $\mathrm{O}_{2}-$ could not be avoided. Although showing an increase of ROS-related proteins, double amiR23.5/23.6 mutants accumulated a considerable amount of $\mathrm{O}_{2} \cdot-$ and $\mathrm{H}_{2} \mathrm{O}_{2}$ indicating that the scavenging system in these plants was probably not enough to reduce the ROS levels.

To estimate the cell death, electrolyte leakage from dead or damaged cells was quantified. According to the results presented in this work, amiR plants appeared to be more disturbed regarding the membrane integrity compared to Col-0 plants. All amiR lines showed increase electrolyte leakage under normal conditions and after being exposed to high temperature. Additionally, trypan blue does not pass through intact cell membranes of live cells, therefore it 
selectively stains dying dead cells and can be used to measure cell death [van Wees, 2008]. Visual analysis revealed deep staining of amiR-T leaves. These assays suggest that the integrity of membranes in amiR lines may be compromised leading to cell death in the triple amiR mutants.

The data described in this work provided evidence of the important roles that SHSPs-M may play not only in the heat response but also in the plant development. Results demonstrate that a functional compensation might be responsible for the phenotype in mutants lacking single sHSPs-M. However, the reduction of the three sHSPs-M produces a profound disruption in the mitochondria and ribosome functionality, severely affects the energy metabolism and the overall cell homeostasis, and leads to alterations in the correct plant development. It remains to be investigated the specific processes in which SHSPs-M are involved and which seem to be disrupted in the amiR-T. Elucidating the role of these SHSPs-M in the mitochondria would also provide a significant contribution to the understanding of the function and regulation of organellar sHSPs. 


\section{CHAPTER III}

\subsection{Mitochondrial small heat shock protein and chilling tolerance in tomato fruit}

Chilling injury $(\mathrm{Cl})$ is a physiological disorder that appears when plants and plant organs are exposed to low, but non-freezing temperatures. In tomato fruit, low temperature is used as a strategy to extend the commercialization period after harvesting. But this procedure can lead to $\mathrm{Cl}$ affecting the production yield and quality. However, genetic variability in the lowtemperature sensitivity is found within different tomato varieties. A comparative study showed contrasting postharvest chilling tolerance between two tomato varieties, cv. Micro-Tom and cv. Minitomato [Gonzalez, 2015]. While cv. Micro-Tom was more tolerant to postharvest chilling, $\mathrm{cv}$. Minitomato showed susceptibility to $\mathrm{Cl}$, demonstrated that $\mathrm{cv}$. Micro-Tom can be considered as a model system to study postharvest chilling tolerance.

It was previously reported the existence of various genes related to different stress responses that may be involved in the acquirement of chilling tolerance [Sanchez-Bel, 2012; CruzMendívil, 2015]. Among them, the sHSP genes may have a role in conferring chilling tolerance. For instance, heat-treated tomato fruit showed acquired tolerance to $\mathrm{Cl}$ and it was related to the accumulation of heat shock proteins [Polenta, 2007]. Proteomic analysis in the fruit of two contrasting tomato lines showed that four SHSPs were increased in the more tolerant genotype in response to cold storage [Page, 2010]. In another proteomic study in tomato fruit stored at chilling and non-chilling temperatures, two sHSPs were up-regulated in response to the cold stress [Sanchez-Bel, 2012]. sHSPs expression after chilling was also analyzed in the pericarp of Micro-Tom and Minitomato fruit. These two varieties exhibited clear different expression of sHSPs, with an increase of sHSPs in Micro-Tom. Interestingly, among them, one mitochondrial sHSPs (sHSP23.8) showed higher expression in Micro-Tom green mature fruit after cold storage. On the contrary, the more susceptible Minitomato fruit showed down-regulation of all sHSPs analyzed during cold storage [Ré, 2016]. These data indicate a potential correlation between the accumulation of sHSP23.8 and the amelioration of chilling symptoms in tomato fruit. Unlike $A$. thaliana where three mitochondrial SHSPs were described [Waters, 2013], tomato has only one mitochondria-located SHSP. Considering that this unique protein may participate in the protection mechanisms against chilling stress, the functional consequences of the down-regulation of SHSP23.8 in tomato fruit was investigated by using knockdown mutants of cv. Micro-Tom. Transgenic fruit was analyzed in their phenotype and in their susceptibility to chilling injury. The results are presented in the current section. 


\subsubsection{Generation of knockdown mutants of SHSP23.8.}

sHSP23.8 gene was targeted and silenced by a specific artificial microRNA (amiR). The amiR design and constructs were developed by Dr. Martin Ré. To focus on the effects on fruit, a fruitspecific promoter (PPC2pro) [Fernandez, 2009] was used to drive the amiR construction. In this study, constructs were used to transform cv. Micro-Tom cotyledons to propagate in vitro and to generate T0 kanamycin-resistant plants. The T1 seeds obtained from these plants were selected in kanamycin-containing MS (1/2) and resistant plants were confirmed by PCR with specific oligonucleotides. The expression reduction of the sHSP23.8 was tested by q-PCR in these $T 1$ transgenics lines. Since a fruit-specific promoter was employed, the q-PCR analysis was performed with cDNA synthesized from green mature fruit. This analysis allowed the identification of several T1 knockdown lines (data not shown) which were propagated to obtain the T2 generation. All experiments showed in this work were performed in the T2 plants, from now on refer to as amiR23.8 lines. Figure 4.32 illustrates the lower expression levels of sHSP23.8 measured by q-PCR in the isolated T2 lines. Green mature fruit from Micro-Tom WT plants was used as a control. The reference gene ribosomal protein L2 (RPL2) was added as a control gene for the quantification. The data obtained from q-PCR experiments were normalized to the basal expression of SHSP23.8 in WT plants.

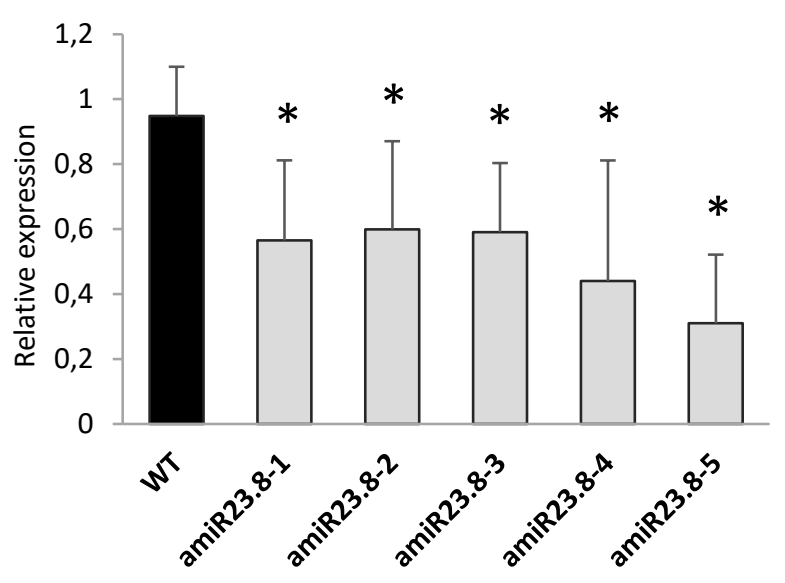

Figure 4.32. Expression of $S H S P 23.8$ in WT and amiR23.8 fruit. Quantitative real-time PCR of $s H S P 23.8$ in Micro-Tom transgenic fruit. Fruit of 5 independent T2 lines were collected at the green mature stage and used for q-PCR determinations. All expression values were first normalized to the RPL2 expression taken as a reference. Relative expression of transcripts was then normalized to their respective expression levels in WT fruit. Each data point consists of the mean value + SD of three biological replicates. Asterisks were used to show statistical significance by one-sided t-test with $p<0.05$. 


\subsection{2. amiR23.8 fruit is more susceptible to chilling injury than WT fruit.}

To evaluate the effects of the down-regulation of SHSP23.8 in the chilling tolerance, transgenic fruit was collected at the green mature stage and evaluated after cold treatment. Fruit ripening was studied in two conditions: on the vine (fruit that ripens on the plant), and prechilled (fruit that was harvested at the green mature stage, stored for 28 days at $4 \stackrel{\circ}{ } \mathrm{C}$, and then transferred back to the climate chamber). Under normal conditions of ripening, transgenic fruit showed WT-like phenotype. Immediately after cold treatment fruit was still green and showed only slight signs of injury. Evidence of $\mathrm{Cl}$ was seen in the following days after being returned to growth temperature. Ripening of fruit was visually checked during 15 days. Although amiR23.8 fruit did not completely decay, they developed visible symptoms of chilling injury after 15 days of ripening (Fig. 4.33). Most of the amiR23.8 fruit failed to ripen normally, they showed wilting and skin wrinkles, partial discoloration and did not reach full red color. Fifteen days after chilling, fruit was internally analyzed by using the "Tomato Analyzer Color Test" to quantify the color parameters $\mathrm{R}$ (red), $\mathrm{G}$ (green), and B (blue) of the RGB color space. Besides this, the $\mathrm{L}^{*}, \mathrm{a}^{*}$ and $b^{*}$ values of the CIELAB color space along with the Hue and Chroma color descriptors were calculated. Color data of WT and 5 amiR23.8 lines are exposed in Table 4.18. Since fruit did not differ in $\mathrm{B}$, the values of this parameter were excluded from the table. Compare to WT, amiR23.8 fruit exhibited lower "Red" and greater "Green" values, which is consistent with more green-yellow fruit as it can be observed in Fig. 4.33-B. The lightness value $L^{*}$ was higher in amiR23.8 fruit indicating the brighter color of the fruit. $a^{*}$ value represents the green-red component: $a^{*}<0$ indicates green and $a^{*}>0$ indicates red. On the other hand, $b^{*}$ represents the blue-yellow component, indicating blue when $b^{*}<0$ and yellow when $b^{*}>0$. As it was expected, amiR23.8 fruit showed lower $\mathrm{a}^{*}$ and higher $\mathrm{b}^{*}$ values compared to WT fruit. Similarly, Hue values were greater in amiR23.8 lines which is associated with the green color. No differences were found in Chroma.

Since a well-known symptom of chilling injury is the loss of water, amiR23.8 and WT fruit was weighed after being harvested and once again directly after the chilling treatment (28 days at $\left.4^{\circ} \mathrm{C}\right)$. The loss of weight was used as an estimation of water loss. Fruit of amiR23.8 showed higher loss of water compared to WT fruit (Fig. 4.34). Furthermore, electrolyte leakage was evaluated in the pericarp of fruit exposed to chilling temperatures and allowed to recover for 1 day in the climate chamber. The pericarp tissue of amiR23.8 pre-chilled fruit showed increased ion leakage compare to WT fruit (Fig. 4.35). The fruit deterioration indicates that knockdown mutants of sHSP23.8 are highly susceptible to cold stress and developed chilling injury symptoms. 


\begin{tabular}{|c|c|c|c|c|c|c|c|}
\hline & Red & Green & $L^{*}$ & $a^{*}$ & $\mathbf{b}^{*}$ & Hue & Chroma \\
\hline WT & 185.56 & 138.54 & 57.23 & 9.41 & 33.93 & 74.35 & 37.40 \\
\hline amiR23.8-1 & $174.37^{*}$ & 149.39 * & 59.46 * & 2.09 * & 36.40 * & 87.26 * & 36.38 \\
\hline amiR23.8-2 & 174.34 * & $153.47^{*}$ & 60.02 * & -1.52 * & 37.12 * & & 7.83 \\
\hline amiR23.8-3 & $177.63^{*}$ & $147.35^{*}$ & $58.75^{*}$ & 2.53 * & 36.37 * & 87.93 * & 37.06 \\
\hline amiR23.8-4 & 178.20 * & 152.30 * & 60.62 * & 1.99 * & 38.78 * & 87.09 * & 39.19 \\
\hline amiR23.8-5 & $173.57^{*}$ & 146.42 * & 58.92 * & 1.68 * & 35.70 * & $87.78^{*}$ & 34.78 \\
\hline
\end{tabular}

Table 4.18. Color parameters of WT and amiR23.8 fruit after chilling treatment. Cross section fruit was used for color analysis through Tomato Analyzer 3.0. Values in the table correspond to the mean value + SD of at least ten biological replicates. Asterisks were employed to indicate statistical significance by one-sided t-test with $\mathrm{p}<0.05$.

A

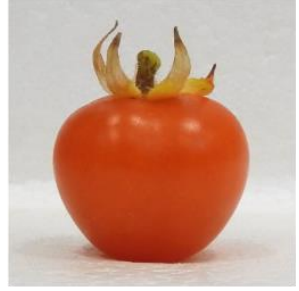

B

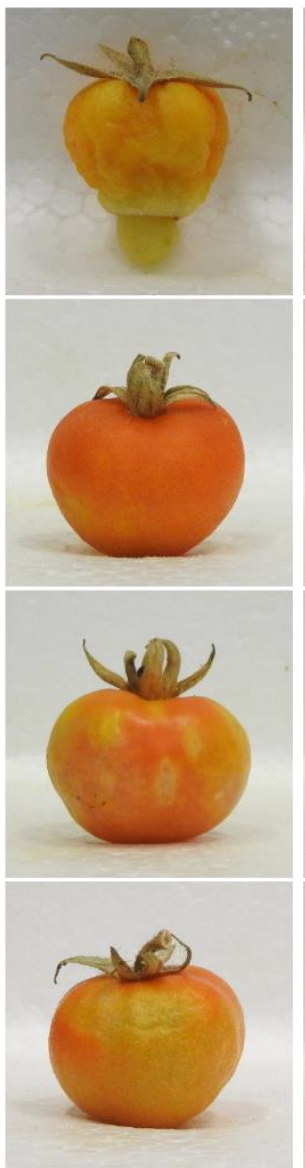

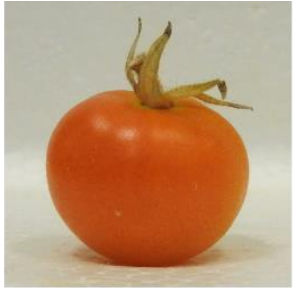
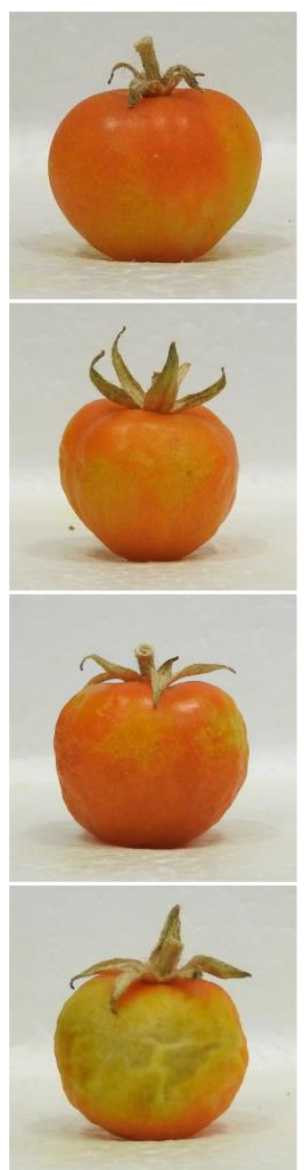
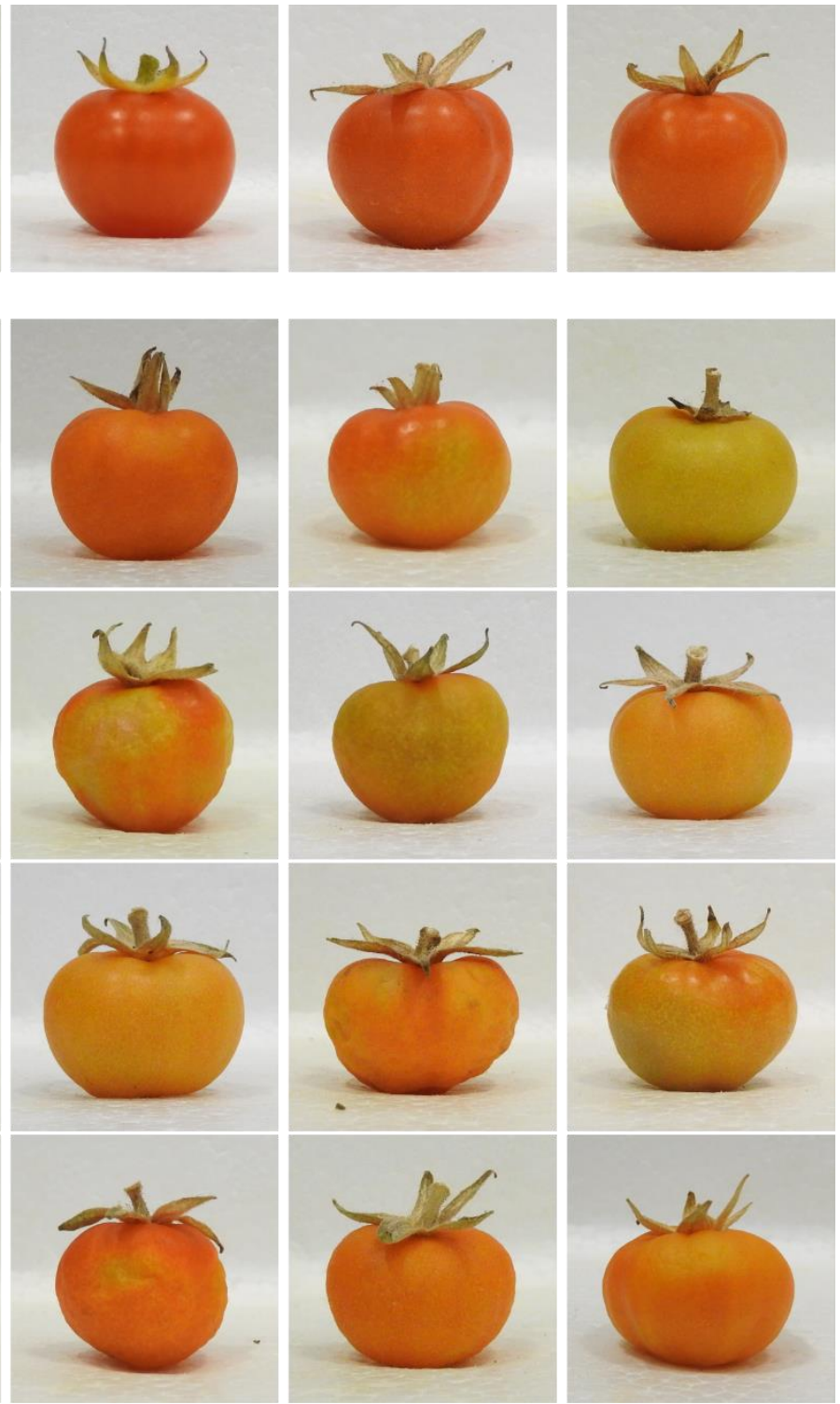

Figure 4.33. Fruit of WT and amiR23.8 plants after chilling treatment. Fruit was harvested at the green mature stage, stored for 28 days at $4 \stackrel{\circ}{\circ} \mathrm{C}$ and transferred to the climate chamber with normal temperature for ripening. Pictures were taken 15 days after chilling. (A) WT fruit. (B) amiR23.8 fruit (4 representative fruits from 5 independent lines). 

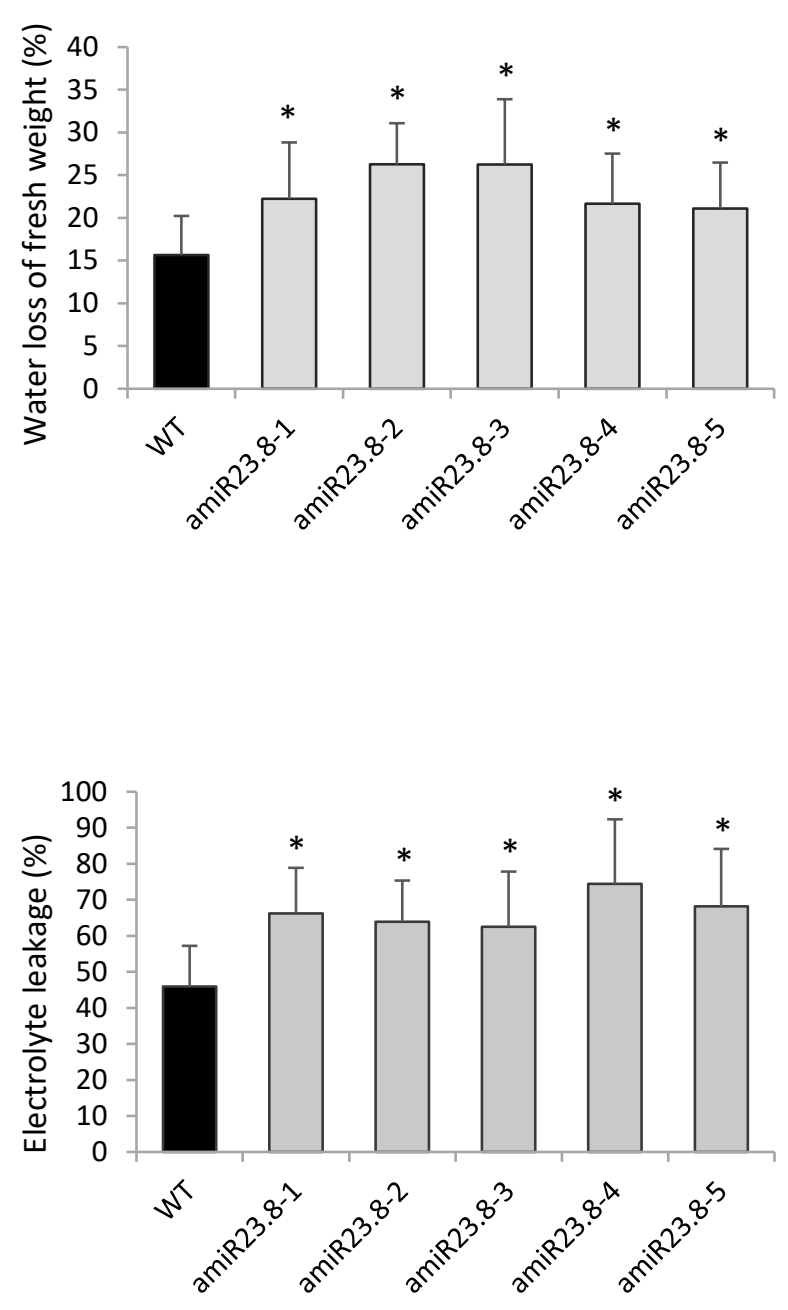

Figure 4.34. Water loss rates of WT and amiR23.8 fruit. Green mature fruit was weighed before and directly after cold storage (28 days at $4{ }^{\circ} \mathrm{C}$ ). Values represent weight losses as a percentage relative to the starting weight. Data point represents the mean value and SD of at least 8 biological replicates. Asterisks mean significance by one-sided t-test with $p<0.05$.
Figure 4.35. Electrolyte leakage in amiR23.8 and WT fruit after chilling treatment. Fruit was collected at the green mature stage and stored at $4{ }^{\circ} \mathrm{C}$ for 28 days before returned to the climate chamber for recovery. Measurements were performed after $24 \mathrm{~h}$ of recovery. Data points correspond to the mean value and SD of at least 4 biological replicates. Asterisks mean significance by one-sided t-test with $\mathrm{p}<0.05$.

\subsubsection{Antioxidant system of amiR23.8 fruit is slightly altered after chilling storage.}

Oxidative stress has been previously linked to the occurrence of chilling injury symptoms in fruit. In this regard, the antioxidant system may play a role in the protection of fruit against chilling [Sala, 1999]. To evaluate the antioxidant capacity of the amiR23.8 and WT fruit, activities of guaiacol peroxidase (GPOX) and catalase (CAT) were measured in pre-chilled fruit (fruit was exposed for 28 days at $4 \stackrel{\circ}{\circ}$ and then allowed to recover for 1 day in the climate chamber). GPOX showed significant changes in fruit of amiR23.8 lines after chilling storage, with lower activity in 8-3 and 8-5 lines and higher activity in 8-2 and 8-4 lines. On the other hand, CAT activity showed significant changes in line 8.1 and not significant changes in the other lines, although a tendency to lower enzyme activity was found in the amiR23.8 mutants (Fig 4.36-B). 


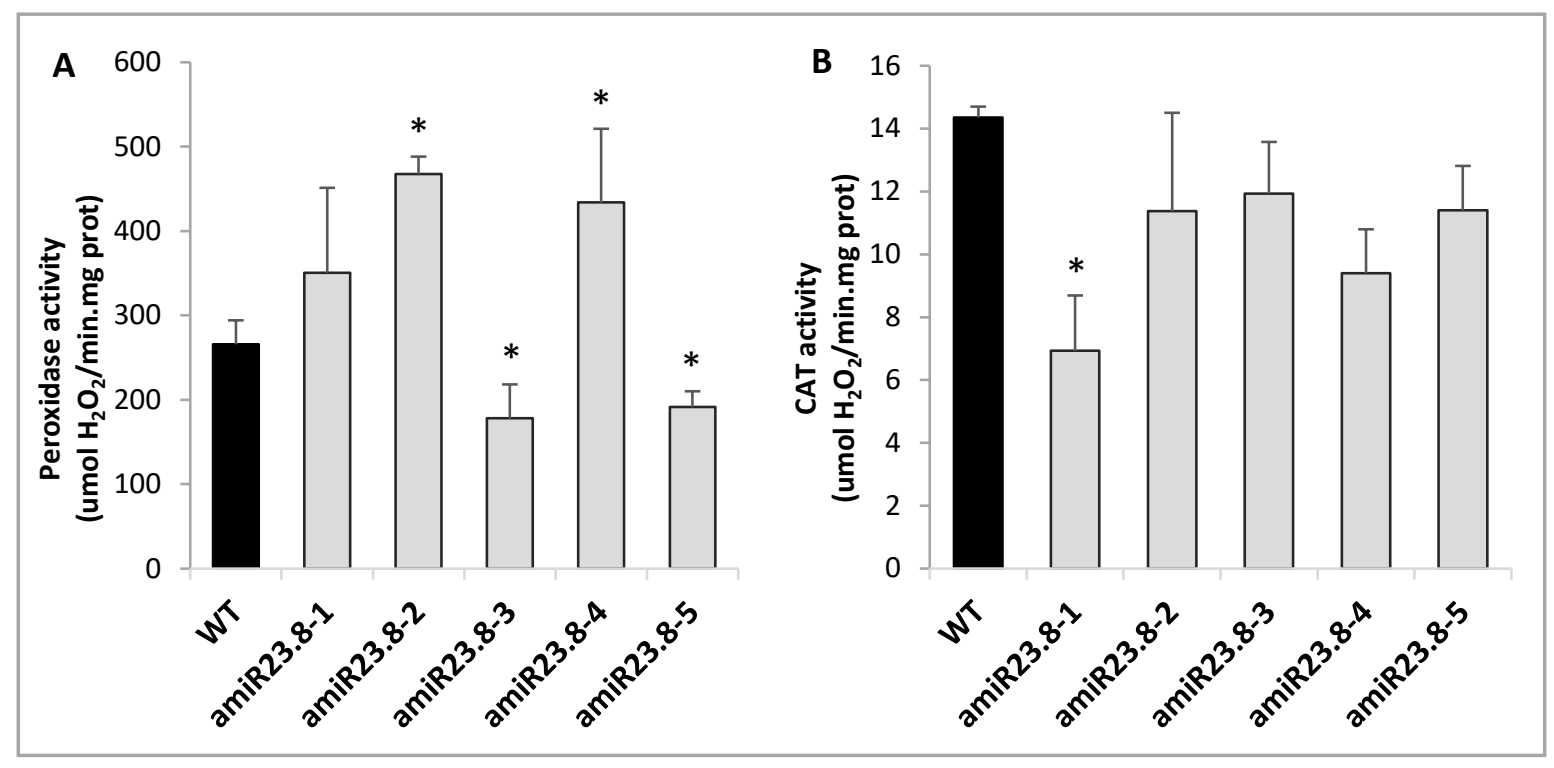

Figure 4.36. Activity of guaiacol peroxidase (A) and catalase (B) in amiR23.8 and WT fruit after chilling treatment. Fruit was collected at the green mature stage and stored at $4{ }^{\circ} \mathrm{C}$ for 28 days before returned to the climate chamber for recovery. Determinations were performed after $24 \mathrm{~h}$ of recovery. Data points correspond to the mean value + SD of three biological replicates. Asterisks were used to indicate significance by one-sided t-test with $\mathrm{p}<0.05$.

\subsubsection{Lipid composition of amiR23.8 fruit after chilling stress differs from WT fruit.}

An UPLC-ESI-MS/MS-based lipid profiling approach was used to investigate changes in the amiR23.8 and WT fruit lipid profiles under normal conditions and after chilling treatment. Fruit lipid profile was analyzed in the pericarp tissue at the stage of green mature and after lowtemperature treatment (fruit harvested at the green mature stage were stored for 28 days at 4 ${ }^{\circ} \mathrm{C}$, and then transferred to the climate chamber for 1 day before pericarp was isolated). Fruit of five independent transgenic lines and two replicates per line were analyzed. Samples consisted of a pool of three fruits from three individual plants. This profile included lipid species belonging to 5 lipid categories, glycerophospholipids, glyceroglycolipids, neutral lipids, sterol lipids, and sphingolipids. Lyso-lipids survey was also performed, although these species were difficult to detect in the samples. Measurements did not include internal standards that can be used as references to determine absolute lipid amounts. Results are shown as relative peak area of every lipid species compared to the total peak area of all species measured in one lipid category. To compare changes happening at the class level relative peak areas of all lipid species of one class were combined. Alterations in lipid categories after chilling stress were also studied by combining the total peak area of all lipid species within one category and by comparing the resulting total areas between WT and amiR23.8 fruit. Data analysis was mainly focused on the differential response of the fruit lipidome to the chilling treatment in WT and amiR23.8 samples separately. 
Lipidomic analysis of tomato fruit after chilling stress revealed alterations in the level of some individual lipid species but no clear patterns were observed. Probably differences in the lipidome response between WT and amiR23.8 fruit can be better observed at a more general scale when combining all lipid species, instead of single lipid species. Therefore, results are mainly presented at the lipid classes and categories levels.

\subsubsection{1. amiR23.8 fruit had altered amounts of glycerolipids and showed differential response to chilling stress.}

The glycerolipidome which includes glycerophospholipids and glyceroglycolipids was evaluated in the amiR23.8 and WT fruit before and after chilling stress. The detected glycerolipids species can be found in Suppl. Fig. 4.5-4.13. Lyso-lipids are molecules with one fatty acid moiety instead of two. Lyso-lipids were measured for all glycerolipid classes, but due to the very low signal intensities, only a few species were used for data analysis (Suppl. Fig 4.14-4.15). In Fig. 4.37 and 4.39 the total levels of the phospholipid classes phosphatidylcholine (PC), phosphatidyl-ethanolamine (PE), phosphatidyl-glycerol (PG), phosphatidylinositol (PI), and phosphatidyl-serine (PS), and glycolipids classes monogalactosyldiacylglycerol (MGDG), digalactosyldiacylglycerol (DGDG), and sulfoquinovosyldiacylglycerols (SQDG) are presented. Since methylation of samples is necessary for PA and lyso-PA measurements, data of these molecules are presented separately (see Suppl. Fig 4.14 and 4.15 for lyso-PA and other lyso-lipids). Compared to control fruit, both amiR23.8 and WT accumulated PC but showed a reduction of PE after chilling stress. Besides this, amiR23.8 fruit exhibited lower levels of PE in control and chilling conditions. Even though PG did not suffer significant changes after cold treatment, the basal levels of these lipids were significantly higher in amiR23.8 fruits. Furthermore, the correlation between specific lipid markers and cold sensitivity of plants has been reported [Zheng, 2016]. That is the case for PG molecules with the composition of 16:0/16:0 which have high melting points. Significant higher amounts of PG (16:0/16:0) were found in amiR23.8 fruit in both normal and treated conditions (Suppl. Fig. 4.7). PI and PS were both reduced in amiR23.8 fruit after being cold-treated while no changes were observed for these molecules in WT fruit. The total peak area of phosphatidic acid (PA) corresponding to 13 detected species was calculated and presented in Fig 4.38 (see Suppl. Fig 4.10 for individual PA species). Interestingly, total PA significantly decreased in WT-treated fruit whereas no level alteration was observed in amiR23.8 fruit after cold treatment. None of the glycolipids showed differential changes in the amiR23.8 treated fruit. After the chilling treatment, WT fruit showed an increase of DGDG and decrease of MGDG molecules, while SQDG did not show significant changes. 


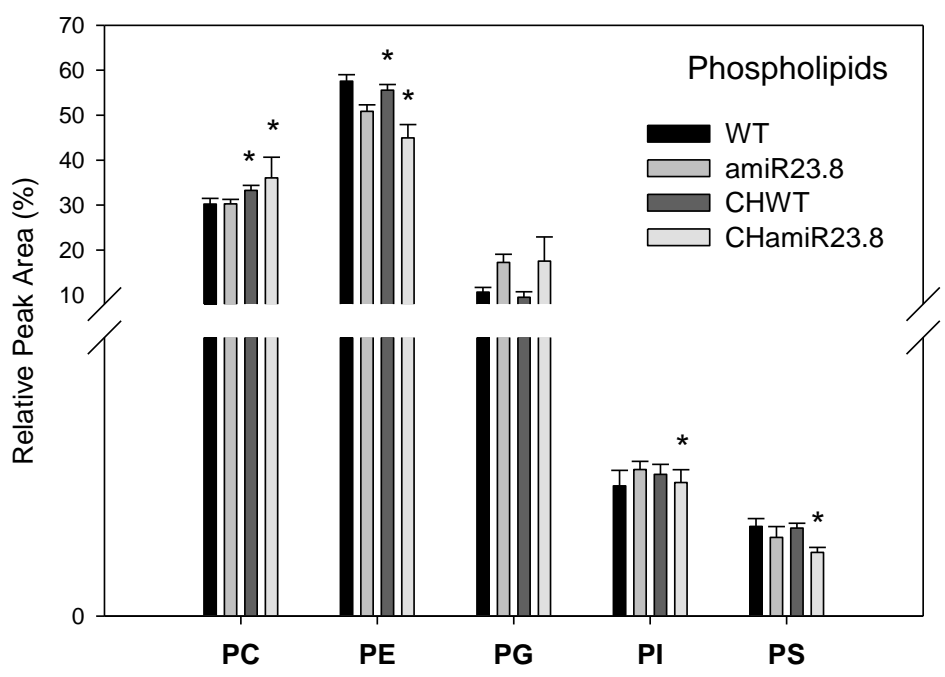

Figure 4.37. Lipid profile of glycerophospholipids in WT and amiR23.8 fruit. Classes include: phosphatidyl-choline (PC), phosphatidyl-ethanolamine (PE), phosphatidyl-glycerol (PG), phosphatidylinositol (PI), and phosphatidyl-serine (PS). Data are shown as the relative peak area of each class compared to the total peak area of all molecules measured in the phospholipid category. Samples of five independent amiR23.8 lines and two replicates per line were analyzed and the 10 samples were then combined to calculate mean and SD values. Six WT samples were also measured and taken as control. Samples consisted of a pool of three fruits from three individual plants. Asterisks indicate significance between normal and chilling conditions by one-way ANOVA, LSD Fisher test with $p<0.05$.

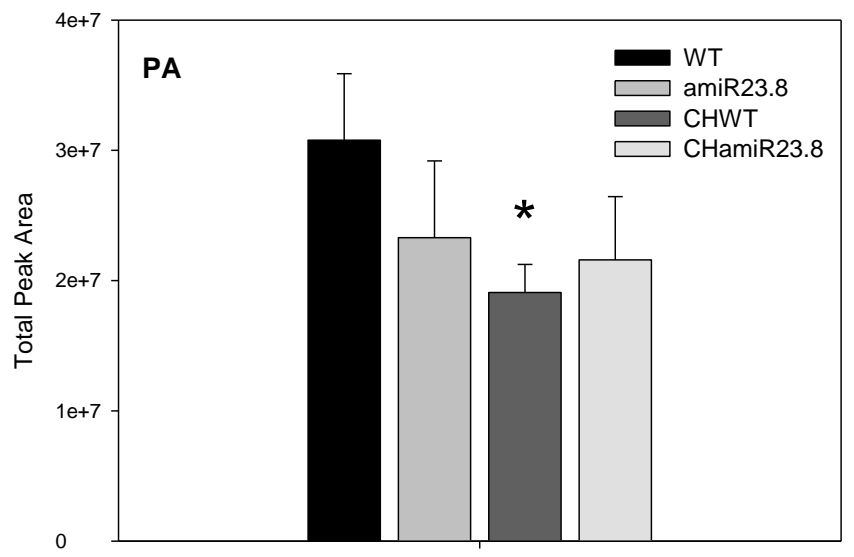

Figure 4.38. Total peak area of phosphatidic acid (PA) in WT and amiR23.8 fruit. Peak areas of all molecules detected in the PA class were combined. Samples of five independent amiR23.8 lines and two replicates per line were analyzed and the 10 samples were then combined to calculate mean and SD values. Six WT samples were also measured and taken as control. Samples consisted of a pool of three fruits from three individual plants. Asterisks mean significance between normal and chilling conditions by one-way ANOVA, LSD Fisher test with $p<0.05$. 


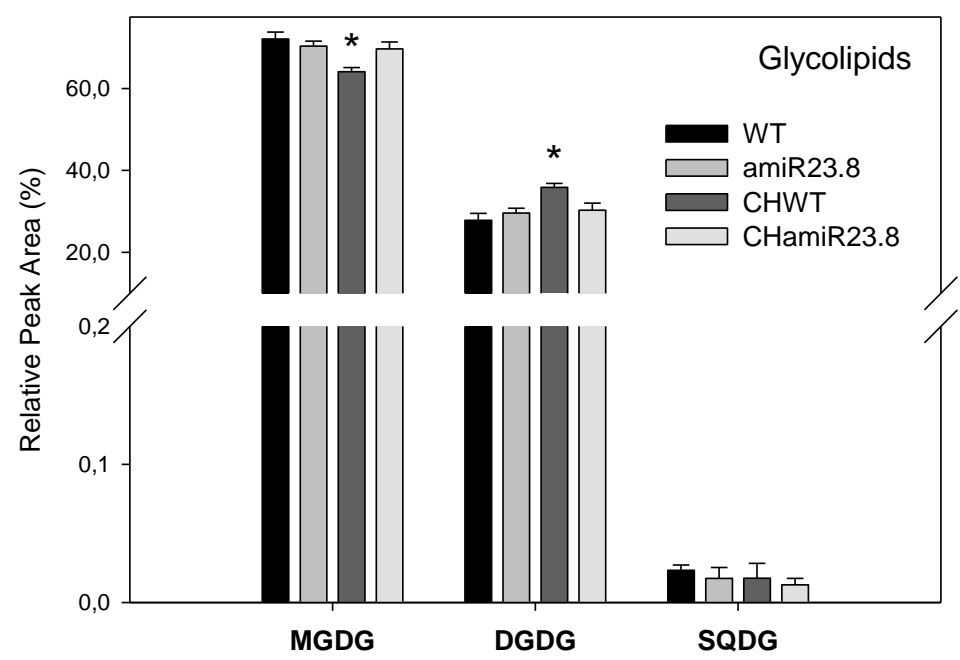

Figure 4.39. Lipid profile of glyceroglycolipids in WT and amiR23.8 fruit. Classes include monogalactosyldiacylglycerol (MGDG), digalactosyldiacylglycerol (DGDG), sulfoquinovosyldiacylglycerol (SQDG). Data are shown as the relative peak area of each class compared to the total peak area of all molecules measured in the glycolipids category. Samples of five independent amiR23.8 lines and two replicates per line were analyzed and the 10 samples were then combined to calculate mean and SD values. Six WT samples were also measured and taken as control. Samples consisted of a pool of three fruits from three individual plants. Asterisks indicate significance between normal and chilling conditions by one-way ANOVA, LSD Fisher test with $p<0.05$.

When considering the total peak area of all phospholipids and glycolipids, an accumulation of total phospholipids in WT and amiR23.8 fruit after chilling stress was observed (Fig. 4.40-A). However, it was higher in WT fruit with a $17 \%$ increase compared to amiR23.8 fruit that showed an increase of only $12 \%$. Total glycolipids, on the other hand, decreased in both WT and amiR23.8 fruit after being exposed to the cold treatment, with $28 \%$ and $24 \%$ fewer glycolipids in WT and amiR23.8, respectively (Fig. 4.40-B). Notably, amiR23.8 fruit exhibited a higher amount of overall glycerolipids in basal and stress conditions. The ratio of phospholipids to glycolipids in green mature fruit was 8.16 in WT and 6.80 in amiR23.8, whereas after coldtreatment it was 13.41 and 10.14 in WT and amiR23.8, respectively. Among glycerolipids, MGDG and DGDG have head groups of different size and the proportion of these molecules influences the integrity of membranes under stress. The same has been assumed to PC and PE molecules. Thus, the ratio of DGDG/MGDG and PC/PE can be useful to evaluate the membrane stability after cold stress. In green mature fruit, the ratio of DGDG/MGDG was 0.38 in WT and 0.42 in amiR23.8. After cold stress, this ratio increased considerably to 0.55 in WT while in amiR23.8 reached only a ratio of 0.43 (Fig. 4.41). The ratio of PC/PE in WT and amiR23.8 was rather similar with 0.52 and 0.59 , respectively. In response to chilling treatment, $\mathrm{PC} / \mathrm{PE}$ increased slightly to 0.59 in WT but it was especially higher in amiR23.8 (ratio of 0.8 ). Double bond index (DBI) and acyl chain length index (ACLI) are good estimators of the degree of unsaturation of glycerolipids and membrane fluidity. A higher DBI and a lower ACLI has been associated with more fluid membranes at low temperatures. To investigate these two 
indexes in the membrane lipids, all glycerolipids in each sample were combined and relative peak areas of each lipid species calculated. The DBI was calculated as $\Sigma[\mathrm{N} \times \mathrm{mol} \%$ lipid] $) / 100$ where $\mathrm{N}$ is the total number of double bonds present in the two fatty acid chains contained in each glycerolipid species. The ACL was considered as $\Sigma[\mathrm{n} \times \mathrm{mol} \%$ lipid])/100 where $\mathrm{n}$ is the total number of carbons in the two fatty acid chains present in each glycerolipid molecule. In addition to this, all glycerolipids with no double bonds in their structure were combined to determine the relative percentage of saturated lipids. In the same way, all glycerolipids with at least one double bond were taken together to estimate the relative percentage of unsaturated membrane lipids. While DBI increased after chilling treatment in both WT and amiR23.8, ACLI showed only a small reduction in amiR23.8 (Table 4.19). Besides this, the basal relative amount of saturated lipids was significantly higher in amiR23.8 fruit compared to WT. In response to chilling, the level of saturated lipids in amiR23.8 decreased, not lower than the level in WT under normal conditions. The opposite was found in the relative percentage of unsaturated lipids, having amiR23.8 fruit significantly lower levels in normal conditions and after chilling.
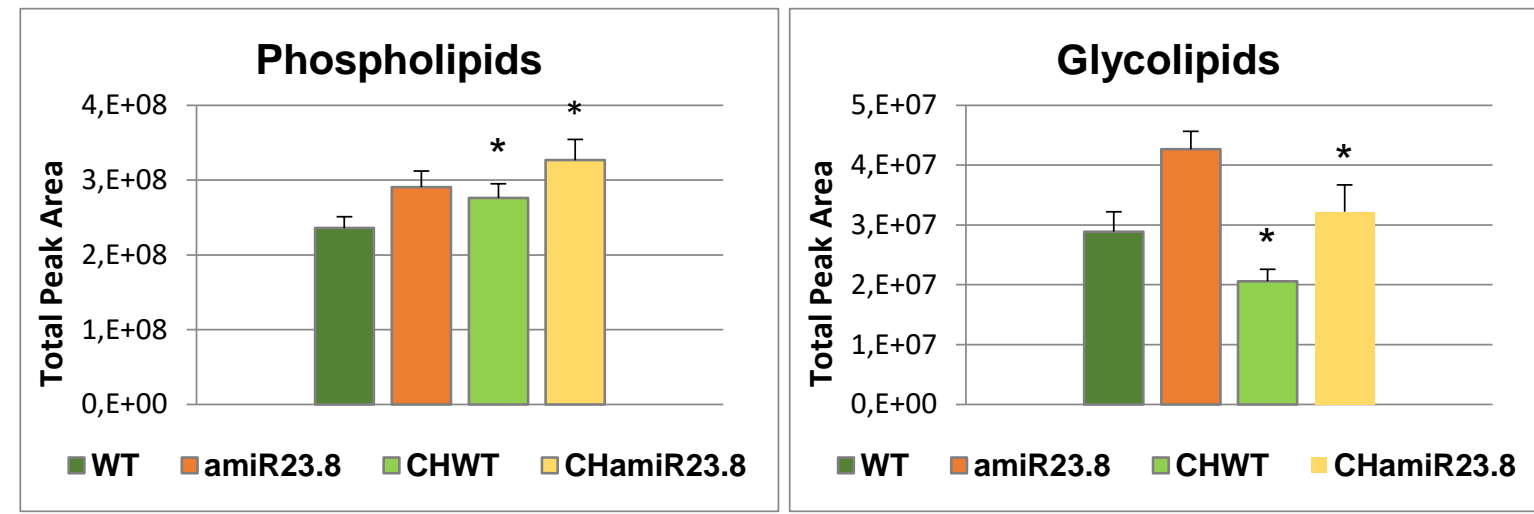

Figure 4.40. Total peak areas of glycerolipids in WT and amiR23.8 fruit. Peak areas of all molecules detected in the phospholipid (A) and glycolipid (B) categories were combined. Samples of five independent amiR23.8 lines and two replicates per line were analyzed and the 10 samples were then combined to calculate mean and SD values. Six WT samples were also measured and taken as control. Samples consisted of a pool of three fruits from three individual plants. Asterisks indicate significance between normal and chilling conditions by one-way ANOVA, LSD Fisher test with $p<0.05$. 


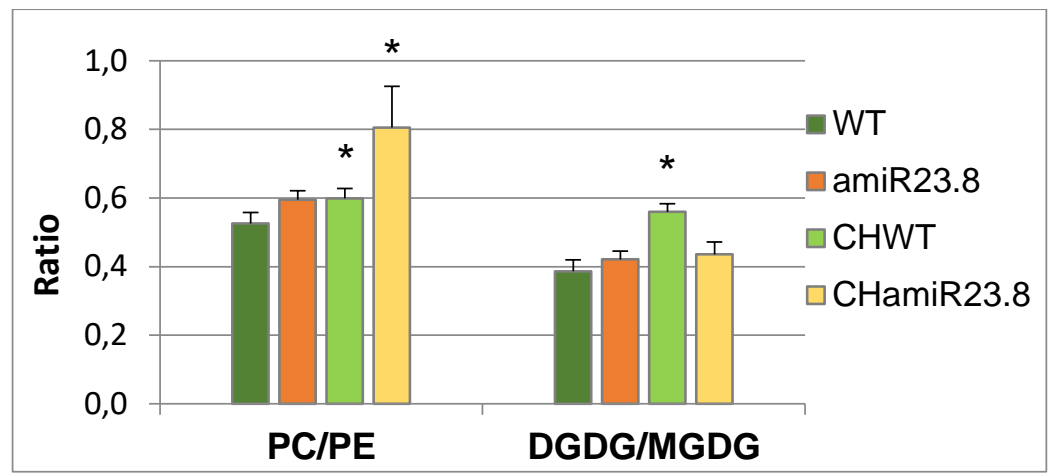

Figure 4.41. PC/PE and DGDG/MGDG in WT and amiR23.8 fruit. Relative peak areas of all molecules detected in each class were combined and ratios were calculated. Samples of five independent amiR23.8 lines and two replicates per line were analyzed and the 10 samples were then combined to calculate mean and SD values. Six WT samples were also measured and taken as control. Samples consisted of a pool of three fruits from three individual plants. Asterisks indicate significance between normal and chilling conditions by one-way ANOVA, LSD Fisher test with $p<0.05$.

\begin{tabular}{lcccc} 
& DBI & ACLI & Saturation (\%) & Unsaturation (\%) \\
\hline WT & $2,86 \mathrm{a}$ & $34,93 \mathrm{ab}$ & $2,46 \mathrm{ab}$ & $97,54 \mathrm{ab}$ \\
\hline amiR23.8 & $2,94 \mathrm{ab}$ & $34,89 \mathrm{a}$ & $4,27 \mathrm{c}$ & $95,73 \mathrm{c}$ \\
\hline CHWT & $3,02 \mathrm{bc}$ & $34,96 \mathrm{~b}$ & $1,44 \mathrm{a}$ & $98,56 \mathrm{a}$ \\
\hline CHamiR23.8 & $3,05 \mathrm{c}$ & $34,83 \mathrm{~d}$ & $2,77^{*} \mathrm{~b}$ & $97,23 \mathrm{~b}$ \\
\hline
\end{tabular}

Table 4.19. Double bond and acyl chain length index of the glycerolipidome of WT and amiR23.8 fruit. All glycerolipids species were combined and used to calculate DBI and ACLI (see formulas in the text). Saturation (\%) and Unsaturation (\%) refer to the relative percentage of all lipid species with no double bond or with at least one double bond in their molecules, respectively. Values in the same column indicated with different letters are significantly different by two-way ANOVA, LSD Fisher test with $p<0.05$.

\subsubsection{Lipidome response of WT and amiR23.8 fruit differed depending on the lipid class.}

Neutral lipids were measured in the lipid classes of diacylglycerides (DAG) and triacylglycerides (TAG), showing no significant changes between WT and amiR23.8 fruit and after the chilling treatment (Fig. 4.42). Sphingolipids are molecules with a backbone of a longchain base (LCB) that can have attached a fatty acid moiety forming a ceramide (Cer). When a sugar moiety is connected, the resulting molecule is a glycosyl-ceramide (GlcCer). On the other hand, when a complex head group consisting of sugars and phosphate is added, the sphingolipid molecule is called glycosylinositolphospho-ceramide (GIPC). In addition to this, free LCBs that do not have a FA attached can be found but only in small amounts. Among the group of sphingolipids, free LCB, Cer, and GlcCer were analyzed. GIPC could not be clearly detected in the fruit samples due to their low signals. Free LCBs were only detected for 18:0;2, 18:0;3 and 18:1;3 (Suppl. Fig. 4.20). Similar sphingolipids response to the cold stress were found in WT and amiR23.8 fruit. Both significantly accumulated Cer and LCB but showed reduced levels of GlcCer (Fig 4.43). Sterol lipids in WT and amiR23.8 fruit were detected as 
the lipid classes sterol-esters (SE), sterol-glycosides (SG) and acylsterol-glycosides (ASG). Sterols molecules consist of a steroid backbone connected in its hydroxyl-group to another residue such as a FA moiety in SE, a sugar moiety in SG or both in ASG. Overall ASG decreased in amiR23.8 fruit while SG accumulated in these mutant samples. SE did not show alterations in response to chilling in WT and amiR23.8 (Fig. 4.44).

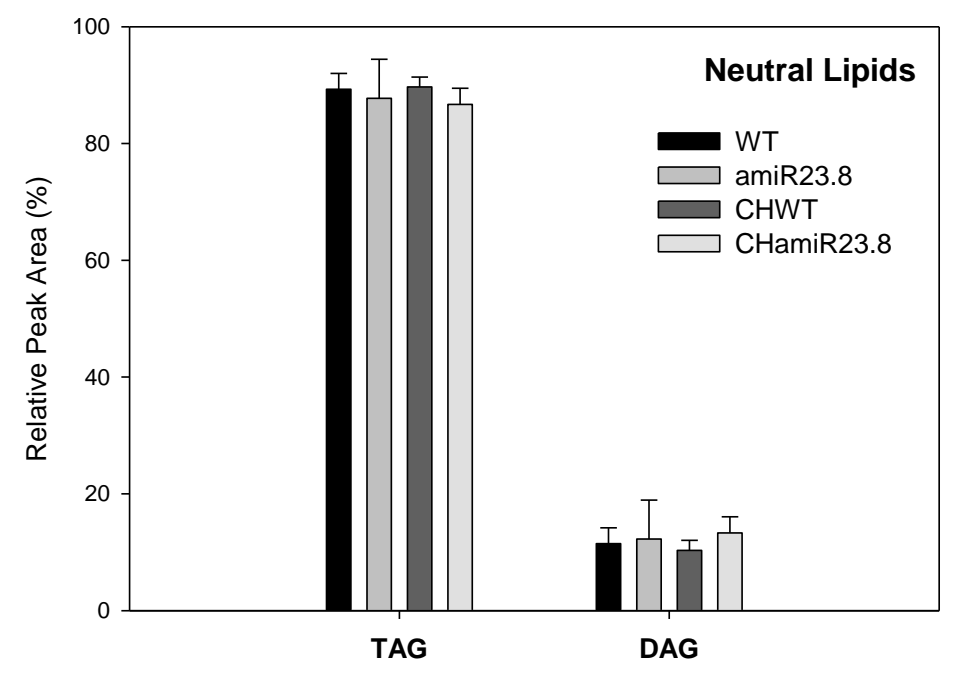

Figure 4.42. Lipid profile of neutral lipids in WT and amiR23.8 fruit. Classes include triacylglycerides (TAG) and diacylglycerides (DAG). Data are shown as the relative peak area of each class compared to the total peak area of all molecules measured in the neutral lipid category. Samples of five independent amiR23.8 lines and two replicates per line were analyzed and the 10 samples were then combined to calculate mean and SD values. Six WT samples were also measured and taken as control. Samples consisted of a pool of three fruits from three individual plants. Asterisks mean significance between normal and chilling conditions by one-way ANOVA, LSD Fisher test with $p<0.05$.

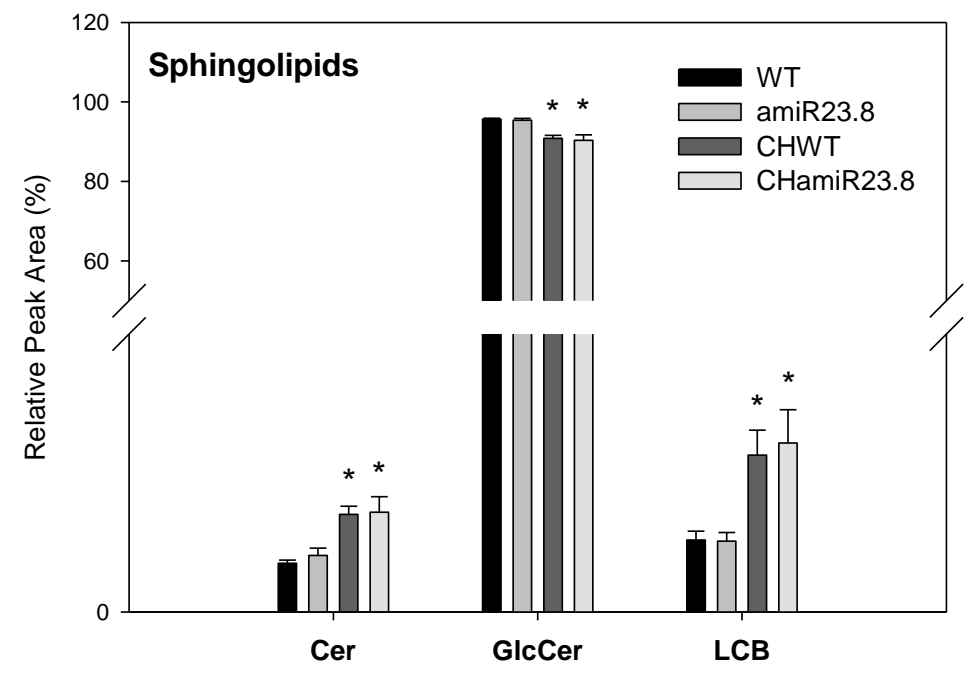

Figure 4.43. Lipid profile of sphingolipids in WT and amiR23.8 fruit. Classes include ceramides (Cer), glucosyl-ceramides (GlcCer) and free LCB. Data are shown as the relative peak area of each class compared to the total peak area of all molecules measured in the sphingolipid category. Samples of five independent amiR23.8 lines and two replicates per line were analyzed and the 10 samples were then combined to calculate mean and SD values. Six WT samples were also measured and taken as control. Samples consisted of a pool of three fruits from three individual plants. Asterisks indicate significance between normal and chilling conditions by one-way ANOVA, LSD Fisher test with $p<0.05$. 


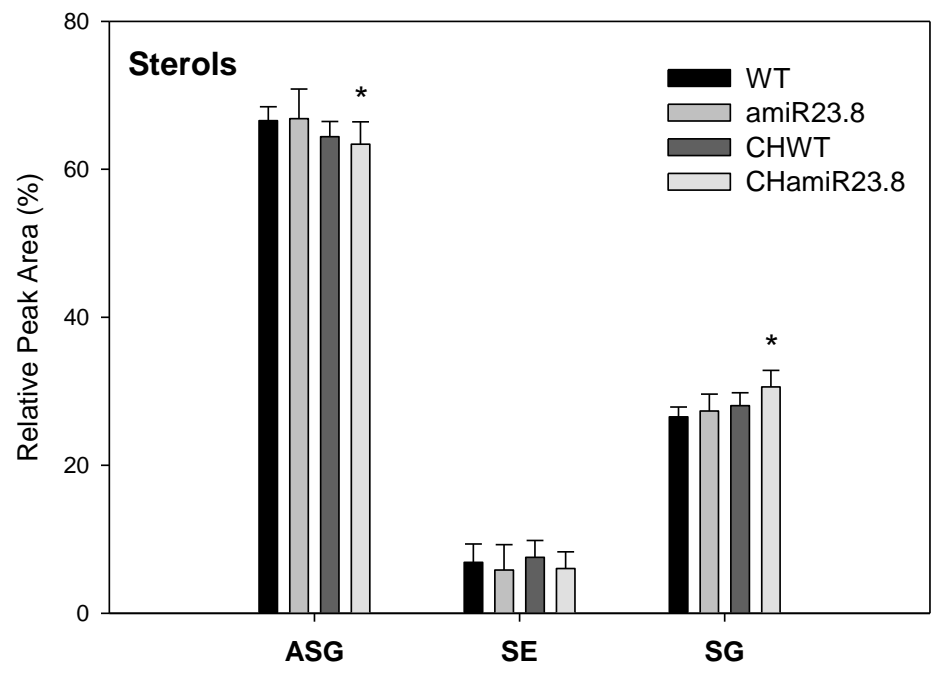

Figure 4.44. Lipid profile of sterolipids in WT and amiR23.8 fruit. Classes include acylsterylglucosides (ASG), sterolester (SE) and sterylglucoside (SG). Data are shown as the relative peak area of each class compared to the total peak area of all molecules measured in the sterol category. Samples of five independent amiR23.8 lines and two replicates per line were analyzed and the 10 samples were then combined to calculate mean and SD values. Six WT samples were also measured and taken as control. Samples consisted of a pool of three fruits from three individual plants. Asterisks indicate significance between normal and chilling conditions by one-way ANOVA, LSD Fisher test with $p<0.05$. 


\subsubsection{Discussion}

The accumulation of small heat shock proteins and acquired chilling tolerance in plants has been subject of several previews reports [Polenta, 2007; Page, 2010; Ré, 2016]. However, the particular functions that SHSPs may have in the response to chilling are largely unknown. In this work, the correlation between the accumulation of the mitochondrial sHSP23.8 and chilling injury resistance in tomato fruit was assessed in a detail that has not been reported to date. The data shown here provide evidence of the protecting role that sHSP23.8 may play against chilling stress.

Fruit of tomato $\mathrm{cv}$. Micro-Tom has been proposed to be tolerant to chilling postharvest injury when comparing with cv. Minitomato, which is susceptible [Gonzalez, 2015]. After chilling, Micro-Tom fruit completes the ripening process and reach the red stage [Malacrida, 2006]. Furthermore, accumulation of the sHSP23.8 was observed in Micro-Tom but not in Minitomato fruit after chilling storage [Ré, 2016]. These results make Micro-Tom a suitable model to study the effects of the down-regulation of SHSP23.8 in the chilling resistance. Knockdown fruit of sHSP23.8 was evaluated in their susceptibility to chilling injury directly after and during several days after chilling treatments. Symptoms of chilling injury were especially evident in amiR23.8 fruit after 15 days of recovery. Compared to WT, amiR23.8 fruit showed partial discoloration, wilting and wrinkles at the surface. Color parameters determination confirmed the more greenyellow color observed in amiR23.8. Most of the amiR23.8 fruit were not able to reach the red color as WT did. Besides this, the loss of water is a common symptom found in tissues after being exposed to cold temperature. Membrane permeability and surface organization of tissues are disrupted due to the extreme temperatures, leading to the greater flux of water through the damaged area and causing fruit dehydration [Lyons, 1973]. amiR23.8 fruit showed higher loss of water and electrolyte leakage after chilling storage, indicating that membrane permeability and stability was especially compromised in this fruit.

Membranes, especially the plasma and chloroplast membranes, can sense external stimuli and are vulnerable to environmental stresses. Several studies have investigated the effect of low temperature in membranes and it has been proposed that membrane damage is the main cause of chilling injury induced by low-temperature in plants [Welti, 2002; Wang, 2006; Zheng, 2016]. Phospholipids and galactolipids are the main glycerolipids and are the principal constituents of plasma and chloroplast membranes, respectively. In order to evaluate the composition and response of amiR23.8 membranes, the complete lipidome of WT and amiR23.8 fruit was evaluated. Previews studies have described the specific changes in membrane composition after exposure to low temperature [Guschina, 2006]. These changes include lipid class alterations, increase in fatty acid unsaturation, chain shortening and the increase of glycerolipids harboring large polar head groups. Alterations at the lipid class levels were found for almost all classes evaluated in the glycerolipidome. After chilling stress, both 
fruits accumulated PC, but only WT fruit accumulated DGDG. PC and DGDG are bilayer lipids with large polar head groups that may increase membrane stability. On the contrary, MGDG and $\mathrm{PE}$ are lipids with small head groups that can lead to the transition to non-bilayer HII-type structures. The decrease of PE was observed in WT and amiR23.8 fruit while MGDG only decreased in WT after chilling stress. Our data demonstrated an increase in the ratio PC/PE in WT and amiR23.8 fruit after chilling, while ratio DGDG/MGDG increased only in WT samples. Increases in the ratio PC/PE and DGDG/MGDG have been previously reported in plants in response to low temperatures and are associated with more stable and fluid membranes [Welti, 2002; Zheng, 2016]. MGDG and DGDG are the major lipids in chloroplast membranes and the adjustment of the ratio DGDG to MGDG in response to chilling stress highly influences the physical state of thylakoid membranes. The fact that DGDG/MGDG did not increase in amiR23.8-treated fruit can be due to the unchanged levels of these two molecules and indicates a disruption in the glycolipid remodeling in response to chilling.

$P G$ is the main phospholipid in plastidic membranes and high levels of these molecules have been previously related to cold sensitivity in plants [Zheng, 2016]. With a high melting point, $P G$ accumulation leads to a decrease in the membrane fluidity [Sakamoto, 2004]. Higher levels of PG were found in amiR23.8 fruit in basal conditions and after chilling. The lipid species PG $32: 0$, which is considered a marker of cold sensitivity, showed especially higher amounts in amiR23.8 fruit. This particular PG composition of amiR23.8 fruit might prevent tolerance to chilling temperature. Although PI functions under freezing stresses are largely unknown, it has been proposed that its accumulation may positively correlate with an enhance chilling tolerance. No PI alterations were observed in WT fruit after chilling. On the contrary, PI was significantly reduced in the more sensitive amiR23.8 fruit. PA are small molecules that show a propensity to form hexagonal II phase in the presence of $\mathrm{Ca}^{2+}$. Increased levels of PA in the membranes of Arabidopsis promotes the non-bilayer phase formation and were associated with less tolerance to freezing [Welti, 2002]. Low temperatures induced an increase of PA level in Arabidopsis and rice, which is then reduced during the recovery period [Zheng, 2016]. After 1 day of recovery, WT fruit suffered a similar reduction in the total levels of PA, which were lower than the amount in basal conditions. amiR23.8 fruit did not show such a reduction indicating an inability to remove PA during recovery that can compromise the membrane integrity.

In addition to this, previews studies have reported that during post-freezing recovery phospholipids increased and glycolipids decreased, probably due to the higher galactolipase activity and hydrolysis in plastidic lipids [Li, 2008]. It was observed in this work that the proportion of phospholipids increased in both WT and amiR23.8 fruit while glycolipids decreased after cold stress. However, data indicated that the increase of phospholipids was smaller and the degradation of glycolipids was lower in amiR23.8 fruit after cold stress. The 
relation between the degree of saturation of membrane lipids and temperature determines the fluidity status of membranes [Zheng, 2011]. A similar response was found in the degree of fatty acid unsaturation in WT and amiR23.8, DBI increased after chilling. The number of saturated lipids decreased while more unsaturated species accumulated after chilling. Increase in the fatty acid unsaturation under cold temperature has also been proved for tomato plants [Spicher, 2016]. Of particular interest was the higher amount of saturated lipid species in amiR23.8 what correlates with less fluid membranes. In addition to the changes in phospholipids and galactolipid contents, a comparable increase in Cer and LCBs, and a decrease in GlcCer contents after cold stress was observed in WT and amiR23.8 fruit. Similar sphingolipids responses were found in leaves of several Arabidopsis accession after cold acclimation [Degenkolbe, 2012]. No considerable modifications in neutral and sterol lipids was observed after chilling, although amiR23.8 showed a slight increase of ASG and SG. Overall, the results presented here indicate a differential degradation of extraplastidic and plastidic lipids in amiR23.8 fruit, and alterations in the remodeling of the lipidome after cold stress, which may lead to higher sensitivity to chilling injury.

In a previews study using tomato Micro-Tom fruit exposed to chilling, it was observed an increase in CAT activity during the first day of recovery. This high antioxidant activity might be responsible for the removal of $\mathrm{H}_{2} \mathrm{O}_{2}$ produced during the first hours after cold stress and it is in concordance with the higher tolerance of Micro-Tom fruit to chilling injury [Malacrida, 2006]. amiR23.8 and WT fruit showed variable alteration in the activity of GPOX and CAT in response to chilling. However, a clear tendency to lower CAT activity was found in amiR23.8 fruit with a significant reduction in one transformant line.

Storage at low temperatures is a common practice used to extend the commercialization period of many important cultivated plants, but it is also responsible for the chilling injury caused by this procedure. The symptoms of the chilling injury highly affect and reduce the fruit quality and consumer acceptability conducting to important economic loss. Elucidating the molecular mechanisms and the key factors beyond chilling tolerance is, in this regard, critical for maintaining fruit quality and diminishing postharvest losses. In this work, the effects of the down-regulation of the mitochondrial SHSP on the chilling resistance of tomato fruit were presented. Micro-Tom fruit with lower levels of sHSP23.8 showed clear symptoms of chilling injury, not only in their external phenotype but also at the plasma membrane and antioxidant system levels. The results discussed here indicate that SHSP23.8 may be directly involved in the protection mechanisms against chilling stress in tomato fruit. 


\section{Conclusions}

The main goal of this work was to understand the role of SHSP-M under stressful conditions in Arabidopsis and tomato.

- The gene pair At5g51430-At5g51440 is head-to-head orientated in the Arabidopsis thaliana genome and they encode a protein that is homologous to Cog7 (EYE), a subunit of the Golgi complex (At5g51430) and a mitochondrial small heat shock protein (sHSP23.5) (At5g51440). They included a bidirectional promoter (446 bp) that is strongly heat induced in the direction of $A t 5 g 51440$ but not considerably stress induced in the other one. Other bidirectional promoters of ACD encoding genes were also analyzed under stress situations, and they were also upregulated by stress conditions. Altogether, these bidirectional promoters have a great potential to be used for biotechnological purposes.

- Three paralogous genes encoding sHSP-M were found in Arabidopsis (At5g51440, At4g25200, and At1g52560). To understand the role of SHSP-M in Arabidopsis, the single, double and triple knock-down mutants were generated by artificial microRNA technology. The triple mutant (amiR23.5/23.6/26.5) showed the most prominent altered phenotype at vegetative and reproductive stages. All amiR mutants displayed alterations in the abundance of several proteins related to translation and to the ribosome functioning and structure. Triple amiR mutant exhibited a higher number of proteins with differential abundance involved in these processes compared to the other single and double amiR23.5/23.6 mutants. Results demonstrated that a functional compensation might be responsible for the phenotype in mutants lacking single sHSPsM. However, the reduction of the three sHSPs-M caused a profound disruption in the mitochondria and ribosome functionality that severely affected the energy metabolism and the overall cell homeostasis, leading to alterations in the correct plant development.

- Unlike A. thaliana, tomato has only one mitochondria-localized sHSP. The functional consequences of the down-regulation of SHSP23.8 in tomato fruit were investigated by using knockdown mutants of cv. Micro-Tom. Transgenic fruit was analyzed in their phenotype and in their susceptibility to chilling injury. Pre-chilled fruit of amiR23.8 mutant showed higher loss of water and increased ion leakage of pericarp tissue compared to WT fruit. The amiR23.8 fruit deterioration indicates that it is highly 
susceptible to cold stress and developed chilling injury symptoms. Differential degradation of extraplastidic and plastidic lipids in amiR23.8 fruit and alterations in the remodeling of the lipidome after cold stress may lead to the high sensitivity of amiR23.8 fruit to chilling injury. The results discussed here indicate that SHSP23.8 may be crucial in the chilling stress tolerance in tomato fruit.

Future studies will be directed to elucidate the physiological substrates of the SHSPs-M and the motif involved in substrate interaction. 


\section{References}

1. Adachi, N., Lieber, M.R. (2002). "Bidirectional gene organization: A common architectural feature of the human genome." Cell. 109: 807-809.

2. Almoguera, C., Coca, M.A., Jordano, J. (1993). "Tissue-specific expression of sunflower heat shock proteins in response to water stress." Plant Journal. 4: 947-958.

3. Armijo, G., Salinas, P., Monteoliva, M., Seguel, A., Garcia, C., Villarroel-Candia, E., Song, W., van der Krol, A., Alvarez, M., Holuigue, L. (2013). "A salicylic acid-induced lectin-like protein plays a positive role in the effector-triggered immunity response of Arabidopsis thaliana to Pseudomonas syringae Avr-Rpm1." Mol. Plant Microbe Interact. 26: 1395-1406.

4. Avelange-Macherel, M. H., Payet, N., Lalanne, D., Neveu, M., Tolleter, D., Burstin, J., Macherel, D. (2015). "Variability within a pea core collection of LEAM and HSP22, two mitochondrial seed proteins involved in stress tolerance." Plant Cell Environ. 38: 12991311.

5. Bailey-Serres, J., Mittler, R. (2006). "The Roles of Reactive Oxygen Species in Plant Cells." Plant Physiology. 141: 311.

6. Bailey, T., Bodén, M., Buske, F., Frith, M., Grant, C., Clementi, L., Ren, J., Li, W., Noble, W. (2009). "MEME SUITE: tools for motif discovery and searching." Nucleic Acids Research. 37: 202-208.

7. Baldwin, A. J., Lioe, H., Robinson, C.V., Kay, L.E., Benesch, J.L. (2011). "aB-crystallin polydispersity is a consequence of unbiased quaternary dynamics." Journal of Molecular Biology. 413: 297-309.

8. Banerjee, J., Kumar Sahoo, D., Dey, N., Houtz, R., Bhushan Maiti, I. (2013). "An Intergenic Region Shared by At4g35985 and At4g35987 in Arabidopsis thaliana Is a Tissue Specific and Stress Inducible Bidirectional Promoter Analysed in Transgenic Arabidopsis and Tobacco Plants." PLOS ONE. 8: e79622.

9. Banzet, N., Richaud, C., Deveaux, Y., Kazmaier, M., Gagnon, J., Triantaphylidés, C. (1998). "Accumulation of small heat shock proteins, including mitochondrial HSP22, induced by oxidative stress and adaptive response in tomato cells." Plant Journal. 13: 519-527.

10. Bartsch, S., Monnet, J., Selbach, K., Quigley, F., Gray, J., von Wettstein, D., Reinbothe, S., Reinbothe, C. (2008). "Three thioredoxin targets in the inner envelope membrane of chloroplasts function in protein import and chlorophyll metabolism." PNAS. 105: 49334938.

11. Basha, E., Friedrich, K.L., Vierling, E. (2006). "The N-terminal arm of small heat shock proteins is important for both chaperone activity and substrate specificity." $\mathrm{J}$ Biol Chem. 281: 39943-39952.

12. Basha, E., Jones, C., Blackwell, A.E., Cheng, G., Waters, E.R., Samsel, K.A., Siddique, M., Pett, V., Wysocki, V., Vierling, E. (2013). "An unusual dimeric small heat shock protein provides insight into the mechanism of this class of chaperones." J Mol Biol. 425: 1683-1696.

13. Basha, E., O'Neill, H., Vierling, E. (2012). "Small heat shock proteins and alphacrystallins: dynamic proteins with flexible functions." Trends Biochem Sci. 37: 106-117.

14. Bates, L. S., Waldran, R.P., Teare, I.D. (1973). "Rapid determination of free proline in water stress studies." Plant and Soil. 39: 205-208.

15. Bernfur, K., Rutsdottir, G., Emanuelsson, C.. (2017). "The chloroplast-localized small heat shock protein Hsp21 associates with the thylakoid membranes in heat-stressed plants." Protein Sci. 26: 1773-1784.

16. Bertani, G. (1951). "Studies on lysogenesis. I. The mode of phage liberation by lysogenic Escherichia coli." J. Bacteriol. 62: 293-300. 
17. Boij, P., Patel, R., Garcia, C., Jarvis, P., Aronsson, H. (2009). "In vivo Studies on the Roles of Tic55-Related Proteins in Chloroplast Protein Import in Arabidopsis thaliana. ." Molecular Plant. 2: 1397-1409.

18. Bondino, H., Valle, E., ten Have, A. (2012). "Evolution and functional diversification of the small heat shock protein/a-crystallin family in higher plants." Planta. 235: 1299-1313.

19. Bondino, H. G., Valle E. M. (2009). "A small intergenic region drives exclusive tissuespecific expression of the adjacent genes in Arabidopsis thaliana." BMC Mol. Biol. 10: 95.

20. Boorstein, W. R., Ziegelhoffer, T., Craig, E.A. (1994). "Molecular evolution of the HSP70 multigene family." J Mol Evol. 38: 1-17.

21. Bréhélin, C., Meye, E., de Souris, J., Bonnard, G., Meyer, Y. (2003). "Resemblance and dissemblance of Arabidopsis type II peroxiredoxins: similar sequences for divergent gene expression, protein localization, and activity." Plant Physiol. 132: 2045-2057.

22. Carroll, A. (2013). "The Arabidopsis Cytosolic Ribosomal Proteome: From form to Function." Front Plant Sci. 4: 1-14.

23. Cartharius, K., Frech, K., Grote, K., Klocke, B., Haltmeier, M., Klingenhoff, A., Frisch, M., Bayerlein, M., Werner, T. (2005). "MatInspector and beyond: promoter analysis based on transcription factor binding sites." Bioinformatics. 21: 2933-2942.

24. Clough, S. J., Bent, A. (1998). "Floral Dip: A simplified method for Agrobacteriummediated transformation of Arabidopsis thaliana." Plant J. 16: 735-743.

25. Colinas, J., Schmidler, S. C., Bohrer, G., Iordanov, B., Benfey, P.N. (2008). "Intergenic and genic sequence lengths have opposite relationships with respect to gene expression." PLoS ONE. 3: e3670.

26. Cruz-Mendívil, A., López-Valenzuela, J. A., Calderón-Vázquez, C. L., Vega-García, M. O., Reyes-Moreno, C., Valdez-Ortiz, A. (2015). "Transcriptional changes associated with chilling tolerance and, susceptibility in 'Micro-Tom' tomato fruit using RNA-Seq." Postharvest Biol. Tec. 99: 141-151.

27. Czechowski, T., Stitt, M., Altmann, T., Udvardi, M.K., and Scheible, W.R. (2005). "Genome-wide identification and testing of superior reference genes for transcript normalization in Arabidopsis." Plant Physiol. 139: 5-17.

28. Chou, M., Chen, Y.-M., Lin, C.-Y. (1989). "Thermotolerance of isolated mitochondria associated with heat shock proteins." Plant Physiol. 98: 617-621.

29. de Miguel, N., Echeverria, P., Angel, S. (2005). "Differential subcellular localization of members of the Toxoplasma gondii small heat shock protein family." Eukaryot Cell. 4: 1990-1997.

30. De Rocher, A. E., Vierling, E . (1994). "Developmental control of small heat shock protein expression during pea seed maturation." The Plant Journal. 5: 93-102.

31. Degenkolbe, T., Giavalisco, P., Zuther, E., Seiwert, B., Hincha, D., Willmitzer, L. (2012). "Differential remodeling of the lipidome during cold acclimation in natural accessions of Arabidopsis thaliana." 72: 972-982.

32. Dhadi, S. R., Krom, N., Ramakrishna, W. (2009). "Genome-wide comparative analysis of putative bidirectional promoters from rice, Arabidopsis and Populus." Gene. 429: 6573.

33. Dixon, D., Davis, B., Edwards, R. (2002). "Functional divergence in the glutathione transferase superfamily in plants. Identification of two classes with putative functions in redox homeostasis in Arabidopsis thaliana." J Biol Chem. 277: 30859-30869.

34. Donahue, J., Alford, S., Torabinejad, J., Kerwin, R., Nourbakhsh, A., Ray, W., Hernick, M., Huang, X., Lyons, B., Hein, P., Gillaspy, G. (2010). "The Arabidopsis thaliana Myoinositol 1-phosphate synthase1 gene is required for Myo-inositol synthesis and suppression of cell death." Plant Cell. 22: 888-903.

35. Downs, C. A., Heckathorn, S.A. (1998). "The mitochondrial small heat-shock protein protects NADH:ubiquinone oxidoreductase of the electron transport chain during heat stress in plants." FEBS Letters. 430: 246-250.

36. Einset, J., Winge, P., Bones, A. (2007). "ROS signaling pathways in chilling stress. ." Plant Signal Behav. 2: 365-367. 
37. Fang, Y., Wang, L., Wang, X., You, Q., Pan, X., Xiao, J., Wang, X. E., Wu, Y., Su, Z., Zhang, W. (2016a). "Histone modifications facilitate the coexpression of bidirectional promoters in rice." BMC Genomics. 17: 768.

38. Fang, Y., Wang, X., Wang, L., Pan, X., Xiao, J., Wang, X. E., Wu, Y., Zhang, W. (2016b). "Functional characterization of open chromatin in bidirectional promoters of rice." Sci Rep. 6: 32088.

39. Fernández-Bautista, N., Domínguez-Núñez, J. A., Moreno, M. C., Berrocal-Lobo, M. (2016). (2016). "Plant Tissue Trypan Blue Staining During Phytopathogen Infection." Bioprotocol. 6: 2078.

40. Fernandez, A., Viron, N., Alhagdow, M., Karimi, M., Jones, M., Amsellem, Z., Sicard, A., Czerednik, A., Angenent, G., Grierson, D. (2009). "Flexible tools for gene expression and silencing in tomato." Plant Physiology. 151: 1729-1740.

41. Feussner, I., Polle, A. (2015). "What the transcriptome does not tell — proteomics and metabolomics are closer to the plants' patho-phenotype." Current Opinion in Plant Biology. 26: 26-31.

42. Finkemeier, I., Goodman, M., Lamkemeyer, P., Kandlbinder, A., Sweetlove, L., Dietz, K. (2005). "The mitochondrial type II peroxiredoxin $\mathrm{F}$ is essential for redox homeostasis and root growth of Arabidopsis thaliana under stress." J Biol Chem. 280: 12168-12180.

43. Fucile, G., Falconer, S., Christendat, D. (2008). "Evolutionary Diversification of Plant Shikimate Kinase Gene Duplicates." PLoS Genet. 4: e1000292.

44. Gamborg, O., Miller, R., Ojima K. (1968). "Nutrient requirement of suspensions cultures of soybean root cells." Exp. Cell Res. 50: 151-158.

45. Garmier, M., Carroll, A., Delannoy, E., Vallet, C., Day, D., Small, I., Millar, A. (2008). "Complex I dysfunction redirects cellular and mitochondrial metabolism in Arabidopsis." Plant Physiol. 148: 1324-1341.

46. Giese, K. C., Basha, E., Catague, B.Y., Vierling, E. (2005). "Evidence for an essential function of the $\mathrm{N}$ terminus of a small heat shock protein in vivo, independent of in vitro chaperone activity." Proceedings of the National Academy of Sciences, USA. 102: 18896-18901.

47. Gill, S., Tuteja, N. (2010). "Reactive oxygen species and antioxidant machinery in abiotic stress tolerance in crop plants." Plant Physiology and Biochemistry. 48: 909-930.

48. Gonzalez, C., Ré, M., Sossi, M., Valle, E., Boggio, S. (2015). "Tomato cv. micro-tom as a model system to study postharvest chilling tolerance." Sci Hortic. 184: 63-69.

49. Gonzalez, C., Zanor, M. I., Ré, M. D., Otaiza, S., Asis, R., Valle, E. M., Boggio, S. B. (2019). "Chilling tolerance of Micro-Tom fruit involves changes in the primary metabolite levels and in the stress response." Postharvest Biology and Technology. 148: 58-67.

50. Graham, D., Patterson, B. D. (1982). "Responses of plants to low, nonfreezing temperatures: proteins, metabolism, and acclimation." Ann Rev Plant Physiol. 33: 347372.

51. Grillitsch, K., Tarazona, P., Klug, L., Wriessnegger, T., Zellnig, G., Leitner, E., Feussner, I., Daum, G. (2014). "Isolation and characterization of the plasma membrane from the yeast Pichia pastoris." Biochimica et Biophysica Acta. 1838: 1889-1897.

52. Grimplet, J., Wheatley, M.D., Jouira, H.B., Deluc, L.G., Cramer, G.R., Cushman, J.C. (2009). "Proteomic and selected metabolite analysis of grape berry tissues under wellwatered and water-deficit stress conditions." Proteomics. 9: 2503-2528.

53. Guschina, I., Harwood, J. (2006). "Mechanisms of temperature adaptation in poikilotherms." FEBS Letters. 580: 5477-5483.

54. Hartl, F. U., Bracher, A., Hayer-Hartl, M. (2011). "Molecular chaperones in protein folding and proteostasis." Nature. 475: 324-332.

55. Haslbeck, M., Vierling, E. (2015). "A First Line of Stress Defense: Small Heat Shock Proteins and their function in protein homeostasis." J Mol Biol. 427: 1537-1548.

56. He, L. H., Chen, J.Y., Kuang, .JF., Lu, W.J. (2012). "Expression of three sHSP genes involved in heat pretreatment-induced chilling tolerance in banana fruit." $\underline{\mathrm{J} \text { Sci Food }}$ Agric. 92: 1924-1930.

57. Herr, D. R., Harris, G.L. (2004). "Close head-to-head juxtaposition of genes favors their coordinate regulation in Drosophila melanogaster." FEBS Letters. 572: 147-153. 
58. Higo, K., Ugawa, Y., Iwamoto, M., Korenaga, T. (1999). "Plant cis-acting regulatory DNA elements (PLACE) database." Nucleic Acids Res. 27: 297-300.

59. Hodges, D. M. (2003). "Postharvest oxidative stress in horticultural crops. ." New York: Food Products Press.

60. Horiguchi, G., Mollá-Morales, A., Pérez-Pérez, J., Kojima, K., Robles, P., Ponce, M., Micol, J., Tsukaya, H. (2011). "Differential contributions of ribosomal protein genes to Arabidopsis thaliana leaf development." Plant J. 65: 724-736.

61. Horwitz, J. (1992). "Alpha-crystallin can function as a molecular chaperone." Proc Natl Acad Sci USA. 89: 10449-10453.

62. Huang, C., Shen, Y., Huang, L., Wu, S., Yeh, C., Lu, C. (2016). "The DEAD-Box RNA Helicase AtRH7/PRH75 Participates in Pre-rRNA Processing, Plant Development and Cold Tolerance in Arabidopsis." Plant Cell Physiol. 57: 174-191.

63. Hüther, C. M., Martinazzo, E. G., Rombaldi, C. V., Bacarin, M. A. (2016). "Effects of flooding stress in 'Micro-Tom' tomato plants transformed with different levels of mitochondrial sHSP23.6." Braz. J. Biol. 77.

64. Hüther, C. M., Ramm, A., Rombaldi, C. V., Bacarin, M. A. (2013). "Physiological response to heat stress of tomato 'MicroTom' plants expressing high and low levels of mitochondrial sHSP23.6 protein." Plant Growth Regulation. 70: 175-185.

65. Ishikawa, T., Machida, C., Yoshioka, Y., Ueda, T., Nakano, A., Machida, Y. (2008). "EMBRYO YELLOW gene, encoding a subunit of the conserved oligomeric Golgi complex, is required for appropriate cell expansion and meristem organization in Arabidopsis thaliana." Genes to Cells. 13: 521-535.

66. Ito, T., Kim, G., Shinozaki, K. (2000). "Disruption of an Arabidopsis cytoplasmic ribosomal protein $\mathrm{S} 13-$ homologous gene by transposon-mediated mutagenesis causes aberrant growth and development." Plant J. 22: 257-264.

67. Jarvis, P. (2008). "Targeting of nucleus-encoded proteins to chloroplasts in plants." 179: 257-285.

68. Jaspard, E., Hunault, G. (2016). "sHSPdb: a database for the analysis of small Heat Shock Proteins." BMC Plant Biol. 16: 135.

69. Jung, H., Okegawa, Y., Shih, P., Kellogg, E., Abdel-Ghany, S., Pilon, M., Sjölander, K., Shikanai, T., Niyogi, K. (2010). "Arabidopsis thaliana PGR7 encodes a conserved chloroplast protein that is necessary for efficient photosynthetic electron transport." PLoS One. 5: e11688.

70. Kader, A. A. (2003). "A Perspective on Postharvest Horticulture (1978-2003)." HortSci 38: 1004-1008.

71. Kersey, P., Allen, J., Allot, A., Barba, M., Boddu, S., Bolt, B.,Carvalho-Silva, D., Christensen, M., Davis, P., Grabmueller, C., Kumar, N., Liu, Z., Maurel, T., Moore, B., McDowall, M., Maheswari, U., Naamati,G., Newman, V., Ong, C., Bolser, D., De Silva, N., Howe, K., Langridge, N., Maslen, G., Staines, D., Yates, A. (2018). "Ensembl Genomes 2018: an integrated omics infrastructure for non-vertebrate species." Nucleic Acids Research. 46: 802-808.

72. Kim, K. P., Yu, J.H., Park, S.M., Koo, H.J., Hong, C.B. (2011). "Tobacco mitochondrial small heat shock protein NtHSP24.6 adopts a dimeric configuration and has a broad range of substrates." BMB Rep. 44: 816-820.

73. Kim, R., Kim, K.K., Yokota, H., Kim, S.H. (1998). "Small heat shock protein of Methanococcus jannaschii, a hyperthermophile." Proc Natl Acad Sci U S A. 95: 91299133.

74. Kotak, S., Larkindale, J., Lee, U., von Koskull-Doring, P., Vierling, E., Scharf, K. (2007). "Complexity of the heat stress response in plants." Curr. Op. Plant. Biol. 10: 310-316.

75. Kovacs, D., Kalmar, E., Torok, Z., Tompa, P. (2008). "Chaperone activity of ERD10 and ERD14, two disordered stress-related plant proteins." Plant Physiol. 147: 381-390.

76. Kriehuber, T., Rattei, T., Weinmaier, T., Bepperling, A., Haslbeck, M., Buchner, J. (2010). "Independent evolution of the core domain and its flanking sequences in small heat shock proteins." FASEB J. 24: 3633-3642. 
77. Krom, N., Ramakrishna, W. (2008). "Comparative analysis of divergent and convergent gene pairs and their expression patterns in rice, Arabidopsis, and Populus." Plant Physiol. 147: 1763-1773.

78. Kumar, S., AlAbed, D., Whitteck, J. T., Chen, W., Bennett, S., Asberry, A., Wang, X., De Sloover, D., Rangasamy, M., Wright, T. R., Gupta, M. (2015). "A combinatorial bidirectional and bicistronic approach for coordinated multi-gene expression in corn." Plant Mol. Biol. 87: 341-353.

79. Lee, G. J., Roseman, A.M., Saibil, H.R., Vierling, E. (1997). "A small heat shock protein stably binds heat-denatured model substrates and can maintain a substrate in a foldingcompetent state." EMBO J. 16: 659-671.

80. Lee, G. J., Vierling, E. (2000). "A Small Heat Shock Protein Cooperates with Heat Shock Protein 70 Systems to Reactivate a Heat-Denatured Protein." Plant Physiol. 122: 189198.

81. Lee, U., Wie, C., Escobar, M., Williams, B., Hong, S.W., Vierling, E. (2005). "Genetic analysis reveals domain interactions of Arabidopsis Hsp100/ClpB and cooperation with the small heat shock protein chaperone system." Plant Cell. 17: 559-571.

82. Leister, D., Wang, X., Haberer, G., Mayer, K. F. X., Kleine, T. (2011). "Intracompartmental and intercompartmental transcriptional networks coordinate the expression of genes for organellar functions." Plant Physiol. 157: 386-404.

83. Lenne, C., Douce, R. (1994). "A low molecular mass heat-shock protein is localized to higher plant mitochondria." Plant Physiol. 105: 1255-1261.

84. Lescot, M., Déhais, P., Thijs, G., Marchal, K., Moreau, Y., Van de Peer, Y., Rouzé, P., Rombauts, S. (2002). "PlantCARE, a database of plant cis-acting regulatory elements and a portal to tools for in silico analysis of promoter sequences." Nucleic Acids Res. 30: 325-327.

85. Li, M., Ji, L., Yang, X., Meng, Q., Guo, S. (2012). "The protective mechanisms of CaHSP26 in transgenic tobacco to alleviate photoinhibition of PSIl during chilling stress." Plant Cell Rep. 31: 1969-1979.

86. Li, W., Wang, R., Li, M., Li, L., Wang, C., Welti, R., Wang, X. (2008). "Differential degradation of extraplastidic and plastidic lipids during freezing and post-freezing recovery in Arabidopsis thaliana." J Biol Chem. 283: 461-468.

87. Lichtenthaler, H. K., Buschmann, C., Rinderle, U., Schmuck, G. (1986). "Application of chlorophyll fluorescence in ecophysiology." Radiation and Environmental Biophysics. 25: 297-298.

88. Lisec, J., Schauer, N., Kopka, J., Willmitzer, L.,Fernie, A.R. (2006). "Gas chromatography mass spectrometry-based metabolite profiling in plants." Nature Protocols. 1: 387-396.

89. Liu, J., Shono, M. (1999). "Characterization of mitochondria-located small heat shock protein from tomato (Lycopersicon esculentum)." Plant Cell Physiol. 40: 1297-1304.

90. Liu, S.-J., Yue, Q.-J., Zhang, W. (2015). "Structural and functional analysis of an asymmetric bidirectional promoter in Arabidopsis thaliana." 57: 162-170.

91. Liu, X., Yang, W., Li, Y., Li, S., Zhou, X., Zhao, Q., Fan, Y., Lin, M., Chen, R. (2016). "The intergenic region of the maize defensin-like protein genes Def1 and Def2 functions as an embryo-specific asymmetric bidirectional promoter." J Exp Bot. 67: 4403-4413.

92. Loewus, F., Murthy, P. (2000). "myo-Inositol metabolism in plants." Plant Science. 150: 1-19.

93. Lubaretz, O., Zur Nieden, U. (2002). "Accumulation of plant small heat-stress proteins in storage organs." Planta. 215: 220-228.

94. Luengwilai, K., Fiehn, O. E., Beckles, D. M. (2010). "Comparison of leaf and fruit metabolism in two tomato (Solanum lycopersicum L.) genotypes varying in total soluble solids." Agric Food Chem. 58: 11790-11800.

95. Lukatkin, A. S., Brazaityte, A., Bobinas, C., Duchovskis, P. (2012). "Chilling injury in chilling-sensitive plants: a review." Agriculture. 99: 111-124.

96. Lund, A. A., Rhoads, D.M., Lund, A.L., Cerny, R.L., Elthon, T.E. (2001). "In vivo modifications of the maize mitochondrial small heat stress protein, HSP22." J Biol Chem. 276: 29924-29929. 
97. Lurie, S., Sabehat, A. (1997). "Prestorage temperature manipulations to reduce chilling injury in tomatoes." Postharvest Biol Technol. 11: 57-62.

98. Lyons, J. M. (1973). "Chilling injury in plants." Annu. Rev. Plant. Physiol. 24: 445-466.

99. Ma, C., Haslbeck, M., Babujee, L., Jahn, O., Reumann, S. (2006). "Identification and Characterization of a Stress-Inducible and a Constitutive Small Heat-Shock Protein Targeted to the Matrix of Plant Peroxisomes." Plant Physiology. 141: 47-60.

100. Malacrida, C., Valle, E.M., Boggio, S.B. (2006). "Postharvest chilling induces oxidative stress response in the dwarf tomato cultivar Micro-Tom." Physiol. Plant. 127: 10-18.

101. Mani, N., Ramakrishna, K., Suguna, K. (2015). "Characterization of rice small heat shock proteins targeted to different cellular organelles." Cell Stress Chaperones. 20: 451-460.

102. Maniatis, T., Fritsch, E., Sambrook, J. (1989). Molecular Cloning. A Laboratory Manual. New York, Cold Spring Harbor Laboratory Press.

103. Maul, F., Sargent, S. A., Sims, C. A., Baldwin, E. A., Balaban, M. O., Huber, D. J. (2000). "Tomato flavor and aroma quality as affected by storage temperature." J Food Sci. 65: 1228-1237.

104. McLoughlin, F., Basha, E., Fowler, M., Kim, M., Bordowitz, J., Katiyar-Agarwal, S., Vierling, E. (2016). "Class I and II Small Heat Shock Proteins Together with HSP101 Protect Protein Translation Factors during Heat Stress." Plant Physiol. 172: 1221-1236.

105. McHaourab, H. S., Godar, J.A., Stewart, P.L. (2009). "Structure and mechanism of protein stability sensors: chaperone activity of small heat shock proteins." Biochemistry. 48: 3828-3837.

106. Meyer, E., Tomaz, T., Carroll, A., Estavillo, G., Delannoy, E., Tanz, S., Small, I., Pogson, B., Millar, A. (2009). "Remodeled respiration in ndufs4 with low phosphorylation efficiency suppresses Arabidopsis germination and growth and alters control of metabolism at night." Plant Physiol. 151: 603-619.

107. Mi, H., Huang, X., Muruganujan, A., Tang, H., Mills, C., Kang, D., Thomas, P. (2016). "PANTHER version 11: expanded annotation data from Gene Ontology and Reactome pathways, and data analysis tool enhancements." Nucl. Acids Res. 45: D183-D189.

108. Minorsky, P. V. (1985). "A heuristic hypothesis of chilling injury in plants: a role for calcium as the primary physiological transducer of injury." Plant, Cell and Environment. 8: 75-94.

109. Mitra, A., Han, J., Zhang, Z.J., Mitra, A. (2009). "The intergenic region of Arabidopsis thaliana cab1 and cab2 divergent genes functions as a bidirectional promoter." Planta. 229: 1015-1022.

110. Mittler, R. (2002). "Oxidative stress, antioxidants and stress tolerance." Trends Plant Sci. 7: 405-410.

111. Mittler, R., Vanderauwera, S., Gollery, M., van-Breusegem, F. (2004). "Reactive oxygen gene network of plants." Trends Plant Sci. 9: 490-498.

112. Mogk, A., Schlieker, C., Friedrich, K.L., Schönfeld, H.J., Vierling, E., Bukau, B. (2003). "Refolding of substrates bound to small Hsps relies on a disaggregation reaction mediated most efficiently by ClpB/DnaK." J. Biol. Chem. 278: 31033-31042.

113. Morrow, G., Inaguma, Y., Kato, K., Tanguay, R.M. (2000). "The small heat shock protein Hsp22 of Drosophila melanogaster is a mitochondrial protein displaying oligomeric organization." J Biol Chem. 275: 1204-1210.

114. Murashige, T., Skoog, F. (1962). "A Revised Medium for Rapid Growth and Bio Assays with Tobacco Tissue Cultures." Physiologia Plantarum. 15: 473-497.

115. Nakamoto, H., Vígh, L. (2007). "The small heat shock proteins and their clients." Cell. Mol. Life Sci. 64: 294-306.

116. Nautiyal, P. C., Shono, M., Egawa, Y. (2005). "Enhanced thermotolerance of the vegetative part of MT-sHSP transgenic tomato line." Scientia Horticulturae. 105: 393409.

117. Neta-Sharir, I., Isaacson, T., Lurie, S., Weiss, D. (2005). "Dual role for tomato heat shock protein 21: protecting photosystem II from oxidative stress and promoting color changes during fruit maturation." The Plant Cell. 17: 1829-1838. 
118. Nishizawa, A., Yabuta, Y., Yoshida, E., Maruta, T., Yoshimura, K., Shigeoka, S. (2006). "Arabidopsis heat shock transcription factor A2 as a key regulator in response to several types of environmental stress." Plant J. 48: 535-547.

119. Novina, C. D., Roy, A. L. (1996). "Core promoters and transcriptional control." Trends Genet. 12: 351-355.

120. Oliveros, J. (2007). "VENNY. An interactive tool for comparing lists with Venn Diagrams.".

121. Page, D., Gouble, B., Valot, B., Bouchet, J., Callot, C., Kretzschmar, A., Causse, M., Renard, C., Faurobert, M. (2010). "Protective proteins are differentially expressed in tomato genotypes differing for their tolerance to low-temperature storage." Planta. 232: 483-500.

122. Parkin, K. L., Marangoni, A., Jackman, R. L., Yada, R. Y., Stanley, D. W. (1989). "Chilling injury. A review of possible mechanisms." J Food Biochem. 13: 127-153.

123. Patel, S., Vierling, E., Tama, F.. (2014). "Replica exchange molecular dynamics simulations provide insight into substrate recognition by small heat shock proteins." Biophys J. 106: 2644-2655.

124. Polenta, G., Calvete, J., González, C. (2007). "Isolation and characterization of the main small heat shock proteins induced in tomato pericarp by thermal treatment." FEBS Journal. 274: 6447-6455.

125. Prasad, T. K., Anderson, M. D., Martin, B. A., Stewart, C. R. (1994). "Evidence for chilling-induced oxidative stress in maize seedlings and a regulatory role for hydrogen peroxide." Plant Cell. 6: 65-74.

126. Qian, W., Miki, D., Lei, M., Zhu, X., Zhang, H., Liu, Y., Li, Y., Lang, Z., Wang, J., Tang, K., Liu, R., Zhu, J.K. (2014). "Regulation of active DNA demethylation by an a-crystallin domain protein in Arabidopsis." Mol Cell. 7: 361-371.

127. Ré, M., Gonzalez, C., Escobar, M., Sossi, M., Valle, E., Boggio, S. (2016). "Small heat shock proteins and the postharvest chilling tolerance of tomato fruit." Physiologia Plantarum. 159: 148-160.

128. Ré, M. D., Gonzalez, C., Sdrigotti, M.A., Sorrequieta, A., Valle, E.M., Boggio, S.B. (2012). "Ripening tomato fruit after chilling storage alters protein turnover." J. Sci. FoodAgric. 92: 1490-1496.

129. Ren, D., Yang, H., Zhang, S. (2002). "Cell death mediated by MAPK is associated with hydrogen peroxide production in Arabidopsis." J Biol Chem. 277: 559-565.

130. Renné, P., Dressen, U., Hebbeker, U., Hille, D., Flügge, U., Westhoff, P., Weber, A. (2003). "The Arabidopsis mutant dct is deficient in the plastidic glutamate/malate translocator DiT2." Plant J. 35: 316-331.

131. Rodriguez, G. R., Moyseenko, J.B., Robbins, M.D., Morejon, N.H., Francis, D.M., van der Knaap, E. (2010). "Tomato analyzer: a useful software application to collect accurate and detailed morphological and colorimetric data from two-dimensional objects." J. Vis. Exp. 37: 1-9.

132. Rugkong, A., Mcquinn, R., Giovannoni, J. J., Rose, J. K. C., Watkins, C. B. (2011). "Expression of ripening-related genes in cold-stored tomato fruit." Postharvest Biol Technol. 61: 1-14.

133. Sabehat, A., Lurie, S., Weiss, D. (1998). "Expression of small heat-shock proteins at low temperatures: A possible role in protecting against chilling injuries." Plant Physiology. 117: $651-658$.

134. Sabehat, A., Weiss, D., Lurie, S. (1996). "The correlation between heat-shock protein accumulation and persistence and chilling tolerance in tomato fruit." Plant Physiology. 110: $531-547$.

135. Sakamoto, A., Sulpice, R., Hou, C.X., Kinoshita, M., Higashi, S.I., Kanaseki, T., Nonaka, H., Moon, B.Y., Murata, N. (2004). "Genetic modification of the fatty acid unsaturation of phosphatidylglycerol in chloroplasts alters the sensitivity of tobacco plants to cold stress." Plant Cell Environ. 27: 99-105.

136. Sala, J. M., Lafuente, M.T. (1999). "Catalase in the Heat-Induced Chilling Tolerance of Cold-Stored Hybrid Fortune Mandarin Fruits." J. Agric. Food Chem. 47: 2410-2414. 
137. Sambrook, J., Russell, D. (2001). Molecular cloning: A Laboratory Manual. New York, Cold Spring Harbor Laboratory Press.

138. Sanchez-Bel, P., Egea, I., Sanchez-Ballesta, M., Sevillano, L., Bolarin, M., Flores, F. (2012). "Proteome changes in tomato fruits prior to visible symptoms of chilling injury are linked to defensive mechanisms, uncoupling of photosynthetic processes and protein degradation machinery." Plant Cell Physiol. 53: 470-484.

139. Sanmiya, K., Suzuki, K., Egawa, Y., Shono, M. (2004). "Mitochondrial small heat-shock protein enhances thermotolerance in tobacco plants." FEBS Letters. 557: 265-268.

140. Sanmiya, K., Suzuki, K., Egawa, Y., Shono, M. (1989). "Mitochondrial small heat-shock protein enhances thermotolerance in tobacco plants." Plant Physiol. 89: 617-621.

141. Scarpeci, T. E., Zanor, M.I., Carrillo, N., Mueller-Roeber, B., Valle, E.M. (2008a). "Generation of superoxide anion in chloroplasts of Arabidopsis thaliana during active photosynthesis: a focus on rapidly induced genes." Plant Molecular Biology. 66: 361378.

142. Scarpeci, T. E., Zanor, M.I., Valle, E.M. (2008b). "Investigating the role of plant heat shock proteins during oxidative stress." Plant Signal Behav. 3: 856-857.

143. Scharf, K., Siddique, M., Vierling, E. (2001). "The expanding family of Arabidopsis thaliana small heat stress proteins and a new family of proteins containing a-crystallin domains (Acd proteins)." Cell Stress Chaperones. 6: 225-237.

144. Scharf, K. D., Berberich, T., Ebersberger, I., Nover, L. (2012). "The plant heat stress transcription factor (Hsf) family: structure, function and evolution." Biochimica et Biophysica Acta. 1819: 104-119.

145. Schauer, N., Steinhauser, D., Strelkov, S., Schomburg, D., Allison, G., Moritz, T. Kopka, J. (2005). "GCMS libraries for the rapid identification of metabolites in complex biological samples." FEBS Letters. 579: 1332-1337.

146. Schwab, R., Ossowski, S., Riester, M., Warthmann, N., Weigel, D. (2006). "Highly specific gene silencing by artificial microRNAs in Arabidopsis." Plant Cell. 18: 1121-1133.

147. Shewfelt, R. L., Rosario, B. A. (2000). "The role of lipid peroxidation in storage disorders of fresh fruits and vegetables." HortSci. 35: 575-579.

148. Shin, R., Kim, M. J., Paek, K. H. (2003). "The CaTin1 (Capsicum annuum TMV-induced Clone 1) and CaTin1-2 Genes are linked head-to-head and share a bidirectional promoter." Plant Cell Physiol. 44: 549-554.

149. Shono, M., Liu, J., Sanmiya, K., Singh, I., Din, J. U., Suzuki, K., Tsukaguchi, T., Egawa, Y. (2002). "Functional analysis of mitochondrial small heat shock protein." JIRCAS Working Report.: 17-23.

150. Siddique, M., Gernhard, S., von Koskull-Döring, P., Vierling, E., Scharf, K-D. (2008). "The plant SHSP superfamily: five new members in Arabidopsis thaliana with unexpected properties." Cell Stress and Chaperones. 13: 183-197.

151. Sievers, F. H., D. (2018). "Clustal Omega for making accurate alignments of many protein sequences." 27: 135-145.

152. Singh, A., Sahi, C., Grover, A. (2009). "Chymotrypsin protease inhibitor gene family in rice: Genomic organization and evidence for the presence of a bidirectional promoter shared between two chymotrypsin protease inhibitor genes." Gene. 428: 9-19.

153. Spicher, L., Glauser, G., Kessler, F. (2016). "Lipid Antioxidant and Galactolipid Remodeling under Temperature Stress in Tomato Plants." Front Plant Sci. 7: 167.

154. Spitzer, C., Reyes, F., Buono, R., Sliwinski, M., Haas, T., Otegui, M. (2009). "The ESCRT-related CHMP1A and B proteins mediate multivesicular body sorting of auxin carriers in Arabidopsis and are required for plant development." Plant Cell. 21: 749-766.

155. Srivastava, R., Liu, J., Howell, S. (2008). "Proteolytic processing of a precursor protein for a growth-promoting peptide by a subtilisin serine protease in Arabidopsis." Plant J. 56: 219-227.

156. Stechmann, A. C.-S., T. (2003). "Phylogenetic analysis of eukaryotes using heat-shock protein Hsp90." J Mol Evol. 57: 408-419.

157. Sun, F., Suen, P., Zhang, Y., Liang, C., Carrie, C., Whelan, J., Ward, J., Hawkins, N., Jiang, L., Lim, B. (2012). "A dual-targeted purple acid phosphatase in Arabidopsis 
thaliana moderates carbon metabolism and its overexpression leads to faster plant growth and higher seed yield." New Phytologist Trust. 194: 206-219.

158. Sun, W., Bernard, C., Van Cotte, B.D., Van Montagu, M., Verbruggen, N. (2001). "AtHSP17.6A, encoding a small heat-shock protein in Arabidopsis, can enhance osmotolerance upon overexpression." Plant Journal. 27: 407-415.

159. Sun, W., van-Montagu, M., Verbruggen, N. (2002). "Small heat shock proteins and stress tolerance in plants." Biochim Biophys Acta. 1577: 1-9.

160. Sun, Y., MacRae, T.H. (2005). "Small heat shock proteins: molecular structure and chaperone function." Cell Mol Life Sci. 62: 2460-2476.

161. Suzuki, N., Mittler, R. (2006). "Reactive oxygen species and temperature stresses: a delicate balance between signaling and destruction." Physiol Plant. 126: 45-51.

162. Swindell, W. R., Huebner, M., Weber, A. P. (2007). "Transcriptional profiling of Arabidopsis heat shock proteins and transcription factors reveals extensive overlap between heat and non-heat stress response pathways." BMC Genomics. 8: 125.

163. Szklarczyk, D., Franceschini, A., Wyder, S., Forslund, K., Heller, D., Huerta-Cepas, J., Simonovic, M., Roth, A., Santos, A., Tsafou, K., Kuhn, M., Bork, P., Jensen, L., von Mering, C. (2015). "STRING v10: protein-protein interaction networks, integrated over the tree of life." Nucleic Acids Res. 43: D447-452.

164. Tao, F., Zhang, L., McCarthy, M.J., Beckles, D.M., Saltveit, M., (2014). "Magnetic resonance imaging provides spatial resolution of chilling injury in Micro-Tom tomato(Solanum lycopersicum L.) fruit." Postharvest Biol. Technol. 97: 62-67.

165. Tarazona, P., Feussner, K., Feussner, I. (2015). "An enhanced plant lipidomics method based on multiplexed liquid chromatography-mass spectrometry reveals additional insights into cold- and drought-induced membrane remodeling." The Plant Journal. 84: 621-633.

166. Textor, S., Bartram, S., Kroymann, J., Falk, K., Hick, A., Pickett, J., Gershenzon, J. (2004). "Biosynthesis of methionine-derived glucosinolates in Arabidopsis thaliana: recombinant expression and characterization of methylthioalkylmalate synthase, the condensing enzyme of the chain-elongation cycle." Planta. 218: 1026-1035.

167. Toivonen, P. (2004). "Postharvest storage procedures and oxidative stress." HortSci. 39: 938-942.

168. Tomaz, T., Bagard, M., Pracharoenwattana, I., Lindén, P., Lee, C., Carroll, A., Ströher, E., Smith, S., Gardeström, P., Millar, A. (2010). "Mitochondrial malate dehydrogenase lowers leaf respiration and alters photorespiration and plant growth in Arabidopsis." Plant Physiol. 154: 1143-1157.

169. Trinklein, N. D., Aldred, S.F., Hartman, S.J., Schroeder, D.I., Otillar, R.P., Myers, R.M. (2004). "An abundance of bidirectional promoters in the human genome." Genome Res. 14: 62-66.

170. Untergasser, A., Cutcutache, I., Koressaar, T., Ye, J., Faircloth, B., Remm, M., Rozen, S. (2012). "Primer3--new capabilities and interfaces." Nucleic Acids Res. 40: e115.

171. Van Aken, O., Ford, E., Lister, R., Huang, S., Millar, A. (2016). "Retrograde signalling caused by heritable mitochondrial dysfunction is partially mediated by ANAC017 and improves plant performance." Plant J. 88: 542-558.

172. Van Aken, O., Zhang, B., Carrie, C., Uggalla, V., Paynter, E., Giraud, E., Whelan, J. (2009). "Defining the Mitochondrial Stress Response in Arabidopsis thaliana." Molecular Plant. 2: 1310-1324

173. van Montfort, R. L., Basha, E., Friedrich, K.L., Slingsby, C., Vierling, E. (2001). "Crystal structure and assembly of a eukaryotic small heat shock protein." Nat Struct Biol. 8: 1025-1030.

174. van Montfort, R. L., Slingsby, C., Vierling, E. (2002). "Structure and function of the small heat shock protein/alpha-crystallin family of molecular chaperones." Adv Protein Chem. 59: 105-156.

175. van Wees, S. (2008). "Phenotypic analysis of Arabidopsis mutants: trypan blue stain for fungi, oomycetes, and dead plant cells." $\underline{\mathrm{CSH} \text { Protoc. }}$ 
176. Volkov, R. A., Panchuk, I.I., Schoffl, F. (2005). "Small heat shock proteins are differentially regulated during pollen development and following heat stress in tobacco." Plant Molecular Biology. 57: 487-502.

177. Wang, C. Y. (2004a). "Chilling and freezing injury the commercial storage of fruits, vegetables, and florist and nursery stocks." Agriculture Handbook. 66.

178. Wang, C. Y. (2010). "Alleviation of chilling injury in tropical and subtropical fruits." Acta Hortic. 864: 267-273.

179. Wang, L., Zhao, C.M., Wang, Y.J., Liu, J. (2005). "Overexpression of chloroplastlocalized small molecular heat-shock protein enhances chilling tolerance in tomato plant." Zhi Wu Sheng Li Yu Fen Zi Sheng Wu Xue Xue Bao. 31: 167-174.

180. Wang, Q., Wan, L., Li, D., Zhu, L., Qian, M., Deng, M. (2009). "Searching for bidirectional promoters in Arabidopsis thaliana." BMC Bioinformatics. 30: 1471-2105.

181. Wang, R., Yan, Y., Zhu, M., Yang, M., Zhou, F., Chen, H., Lin, Y. (2016). "Isolation and Functional Characterization of Bidirectional Promoters in Rice." Frontiers in Plant Science. 7: 766.

182. Wang, W., Vinocur, B., Shoseyov, O., Altman, A. (2004b). "Role of plant heat-shock proteins and molecular chaperones in the abiotic stress response." Trends Plant Sci. 9: 244-252.

183. Wang, X., Li, W., Li, M., Welti, R. (2006). "Profiling lipid changes in plant response to low temperatures." 126: 90-96.

184. Waters, E., Nguyen, S., Eskandar, R., Behan, J., Sanders-Reed, Z. (2008a). "The recent evolution of a pseudogene: diversity and divergence of a mitochondria-localized small heat shock protein in Arabidopsis thaliana." Genome. 51: 177-186.

185. Waters, E. R. (1995). "The molecular evolution of the small heat-shock proteins in plants." Genetics. 141: 785-795.

186. Waters, E. R. (2013). "The evolution, function, structure, and expression of the plant sHSPs." J Exp Bot. 64: 391-403.

187. Waters, E. R., Aevermann, B.D., Sanders-Reed, Z. (2008b). "Comparative analysis of the small heat shock proteins in three angiosperm genomes identifies new subfamilies and reveals diverse evolutionary patterns." Cell Stress \& Chaperones. 13: 127-142.

188. Waters, E. R., Lee, G.J., Vierling, E. (1996). "Evolution, structure and function of the small heat shock proteins in plants." Journal of Experimental Botany. 47: 325-338.

189. Watkins, C. B., Picton, S., Grierson, D. (1990). "Stimulation and inhibition of expression of ripening-related mRNAs in tomatoes as influenced by chilling temperatures." J Plant Physiol. 136: 318-323.

190. Wehmeyer, N., Hernandez, L.D., Finkelstein, R.R., Vierling, E. (1996). "Synthesis of small heat-shock proteins is part of the developmental program of late seed maturation." Plant Physiol. 12: 747-757.

191. Wei, W., Pelechano, V., Järvelin, A., Steinmetz, L. (2011). "Functional consequences of bidirectional promoters." Trends in Genetics. 27: 267-276.

192. Weigel, D., Glazebrook, J. (2002). Arabidopsis: A Laboratory Manual. New York, Cold Spring Harbor Laboratory Press.

193. Welti, R., Li, W., Li, M., Sang, Y., Biesiada, H., Zhou, H.E., Rajashekar, C., Williams, T., Wang, X. (2002). "Profiling membrane lipids in plant stress responses: Role of phospholipase Da in freezing-induced lipid changes in Arabidopsis." J Bio Chem. 277: 1994-2002.

194. Williams, E. J., Bowles, D.J. (2004). "Coexpression of neighboring genes in the genome of Arabidopsis thaliana." Genome Res. 14: 1060-1067.

195. Winter, D., Vinegar, B., Ammar, R., Nahal, H., Wilson, G.V., Provart, N. (2007). "An 'Electronic Fluorescent Pictograph' Browser for Exploring and Analyzing Large-Scale Biological Data Sets." PLoS ONE. 2: e718.

196. Xu, Z., Wei, W., Gagneur, J., Perocchi, F., Clauder-Munster, S., Camblong, J., Guffanti, E., Stutz, F., Huber, W., Steinmetz, L.M. (2009). "Bidirectional promoters generate pervasive transcription in yeast." Nature. 457: 1033-1037. 
197. Yang, X., Winter, C. M., Xia, X., Gan, S. (2011a). "Genome-wide analysis of the intergenic regions in Arabidopsis thaliana suggests the existence of bidirectional promoters and genetic insulators." Current Topics in Plant Biology. 12: 15-33.

198. Yang, X., Winter, C., Xia, X., Gan, S. (2011b). "Genome-wide analysis of the intergenic regions in Arabidopsis thaliana suggests the existence of bidirectional promoters and genetic insulators. ." Current Topics in Plant Biology. 12: 15-33.

199. Yi, S.-Y., Sun, A.-Q., Sun, Y., Yang, J.-Y., Zhao, C.-M., Liu, J. (2006). "Differential regulation of LeHsp23.8 in tomato plants: Analysis of a multiple stress-inducible promoter." Plant Sci. 171: 398-407.

200. Youssef, A., Laizet, Y., Block, M., Maréchal, E., Alcaraz, JP., Larson, T., Pontier, D. Gaffé, J., Kuntz, M. (2010). "Plant lipid-associated fibrillin proteins condition jasmonate production under photosynthetic stress." The Plant Journal. 61: 436-445.

201. Zhang, L., Becker, D. (2015). "Connecting proline metabolism and signaling pathways in plant senescence." Front Plant Sci. 6: 552.

202. Zhang, T., Marand, A., Jiang, J. (2016). "PlantDHS: a database for DNase I hypersensitive sites in plants." Nucleic Acids Research. 44: 1148-1153.

203. Zhao, Y., Xie, S., Li, X., Wang, C., Chen, Z., Lai, J., Gong, Z. (2014). "REPRESSOR OF SILENCING5 Encodes a Member of the Small Heat Shock Protein Family and Is Required for DNA Demethylation in Arabidopsis." Plant Cell. 26: 2660-2675.

204. Zheng, G., Li, L., Li, W. (2016). "Glycerolipidome responses to freezing- and chillinginduced injuries: examples in Arabidopsis and rice." BMC Plant Biol. 16: 70.

205. Zheng, G., Tian, B., Zhang, F., Tao, F., Li, W. (2011). "Plant adaptation to frequent alterations between high and low temperatures: remodelling of membrane lipids and maintenance of unsaturation levels." Plant. Cell Environ. 34: 1431-1442. 


\section{Supplemental data}

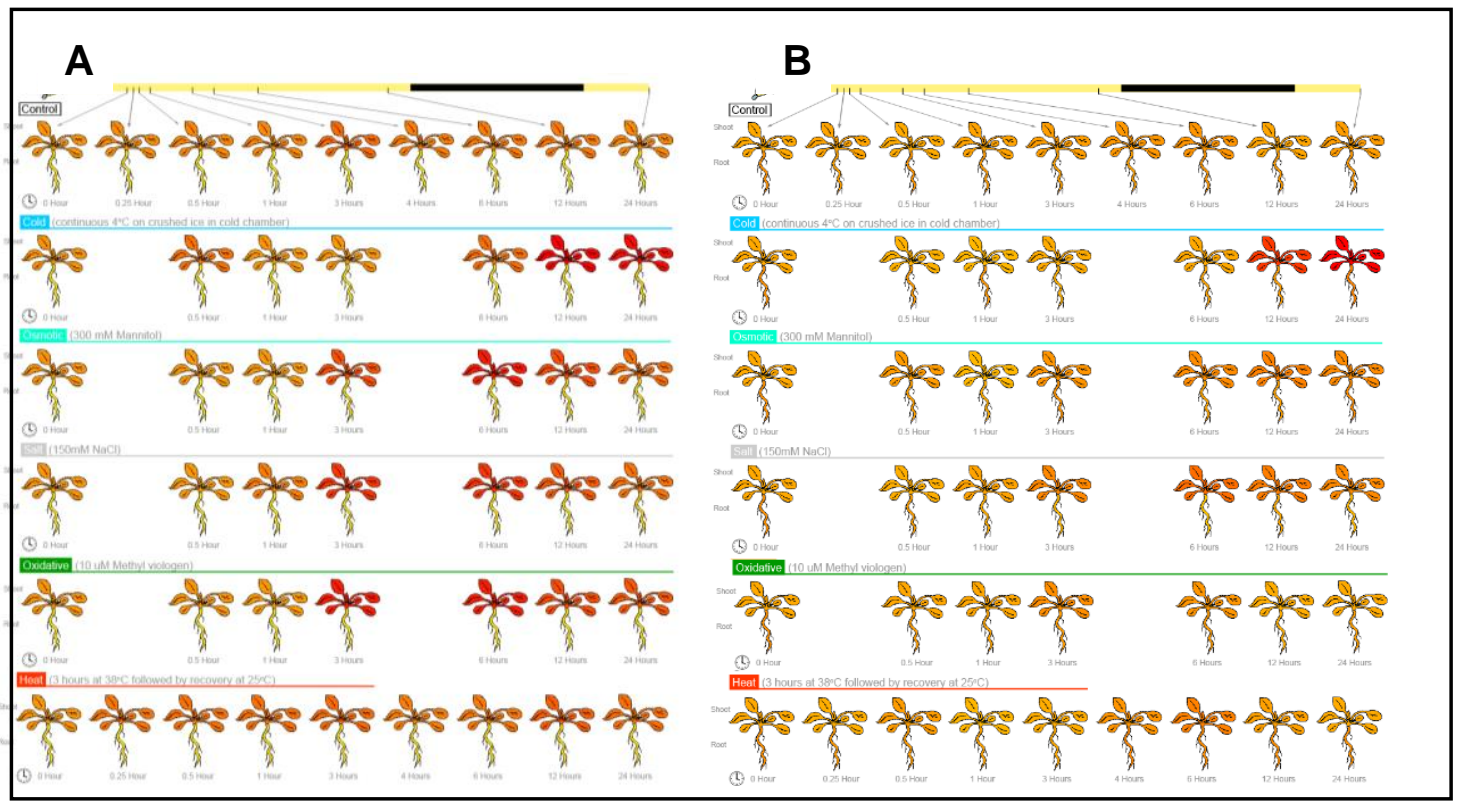

Supplemental figure 4.1. Expression of At4g25650 and At4g25660 after abiotic stresses. Gene expression analysis after cold, osmotic, salt, oxidative and heat stress, from 0 to 24 hours after the stimulus. A) Expression pattern of At4g25650. B) Expression pattern of At4g25660. The graphics were obtained from Arabidopsis eFP-Browser [Winter, 2007]. Access 19.11.2018.

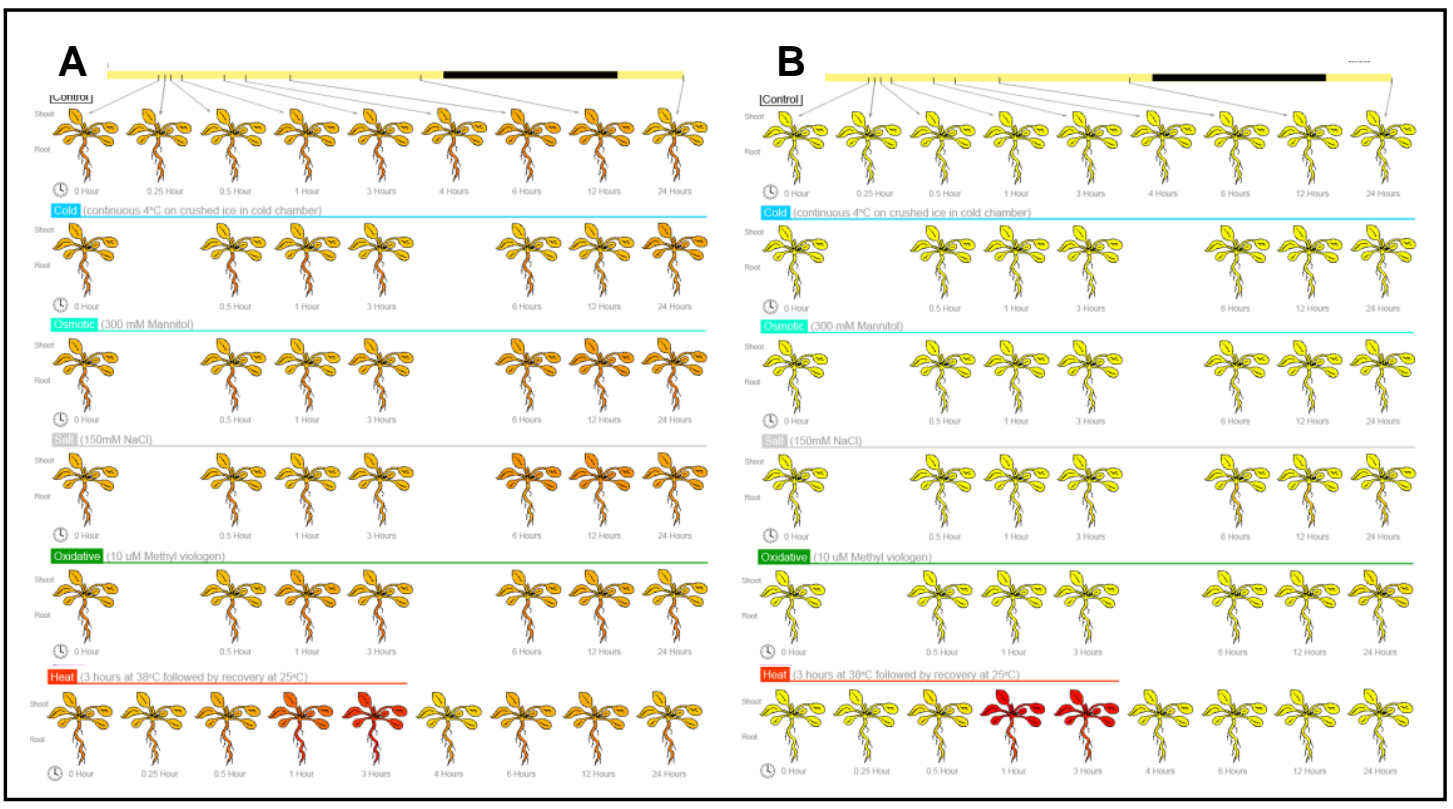

Supplemental figure 4.2. Expression of $A t 5 g 51430$ and $A t 5 g 51440$ after abiotic stresses. Gene expression analysis after cold, osmotic, salt, oxidative and heat stress, from 0 to 24 hours after the stimulus. A) Expression pattern of At5g51430. B) Expression pattern of At5g51440. The graphics were obtained from Arabidopsis eFP-Browser [Winter, 2007]. Access 19.11.2018. 


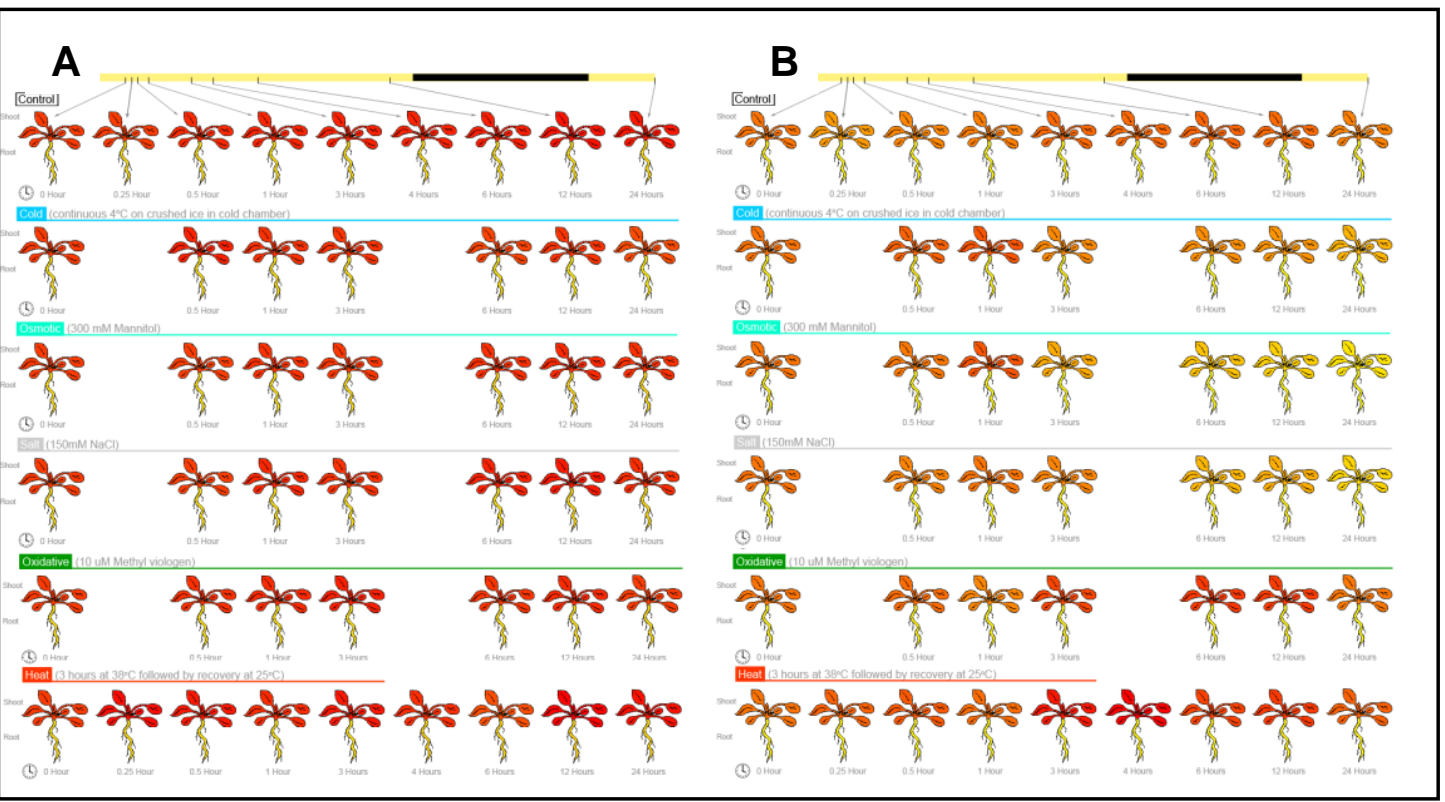

Supplemental figure 4.3. Expression of At2g35490 and At2g35500 after abiotic stresses. Gene expression analysis after cold, osmotic, salt, oxidative and heat stress, from 0 to 24 hours after the stimulus. A) Expression pattern of At2g35490. B) Expression pattern of At2g35500. The graphics were obtained from Arabidopsis eFP-Browser [Winter, 2007]. Access 19.11.2018.

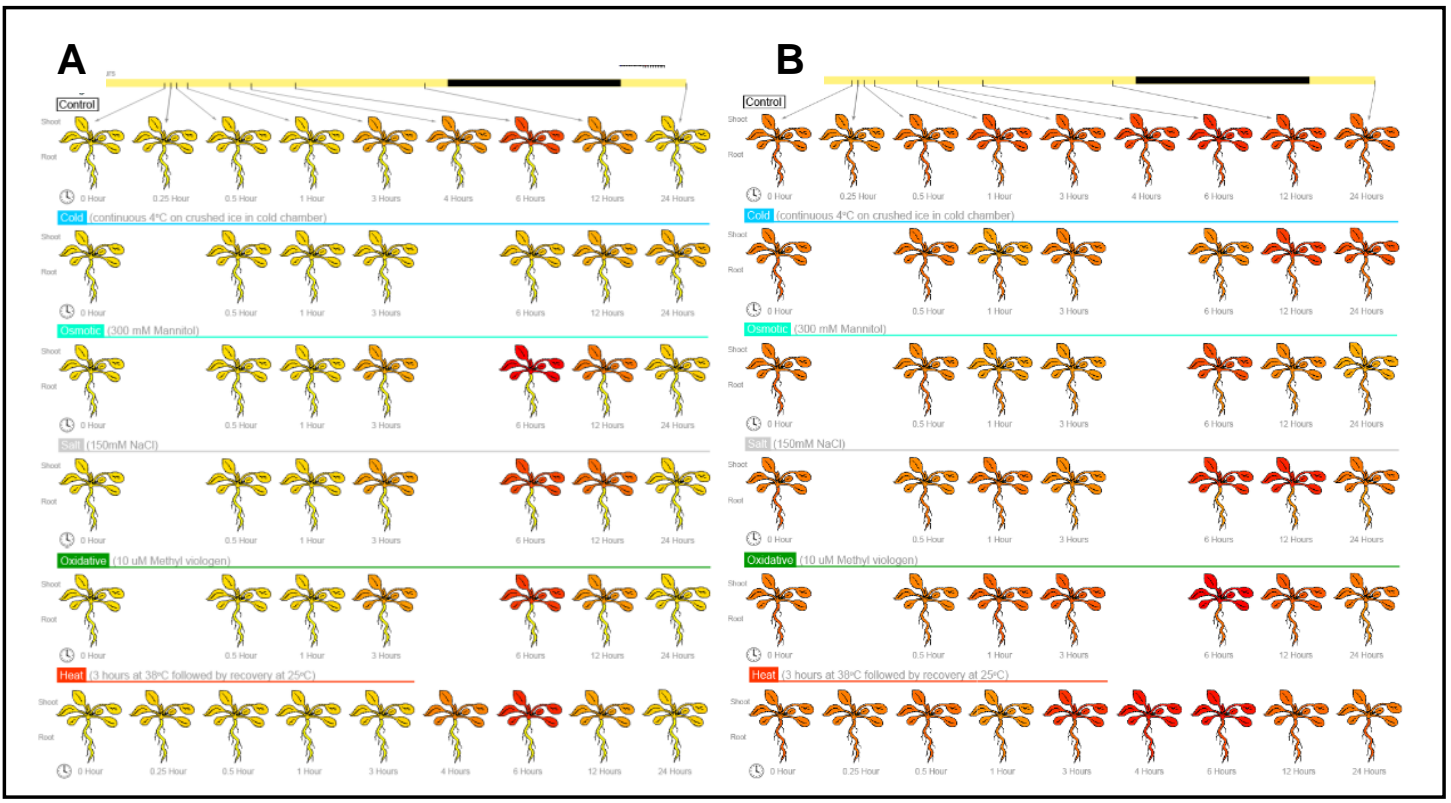

Supplemental figure 4.4. Expression of At1g06460 and At1g06470 after abiotic stresses. Gene expression analysis after cold, osmotic, salt, oxidative and heat stress, from 0 to 24 hours after the stimulus. A) Expression pattern of At1g06460. B) Expression pattern of At1g06470. The graphics were obtained from Arabidopsis eFP-Browser [Winter, 2007]. Access 19.11.2018. 


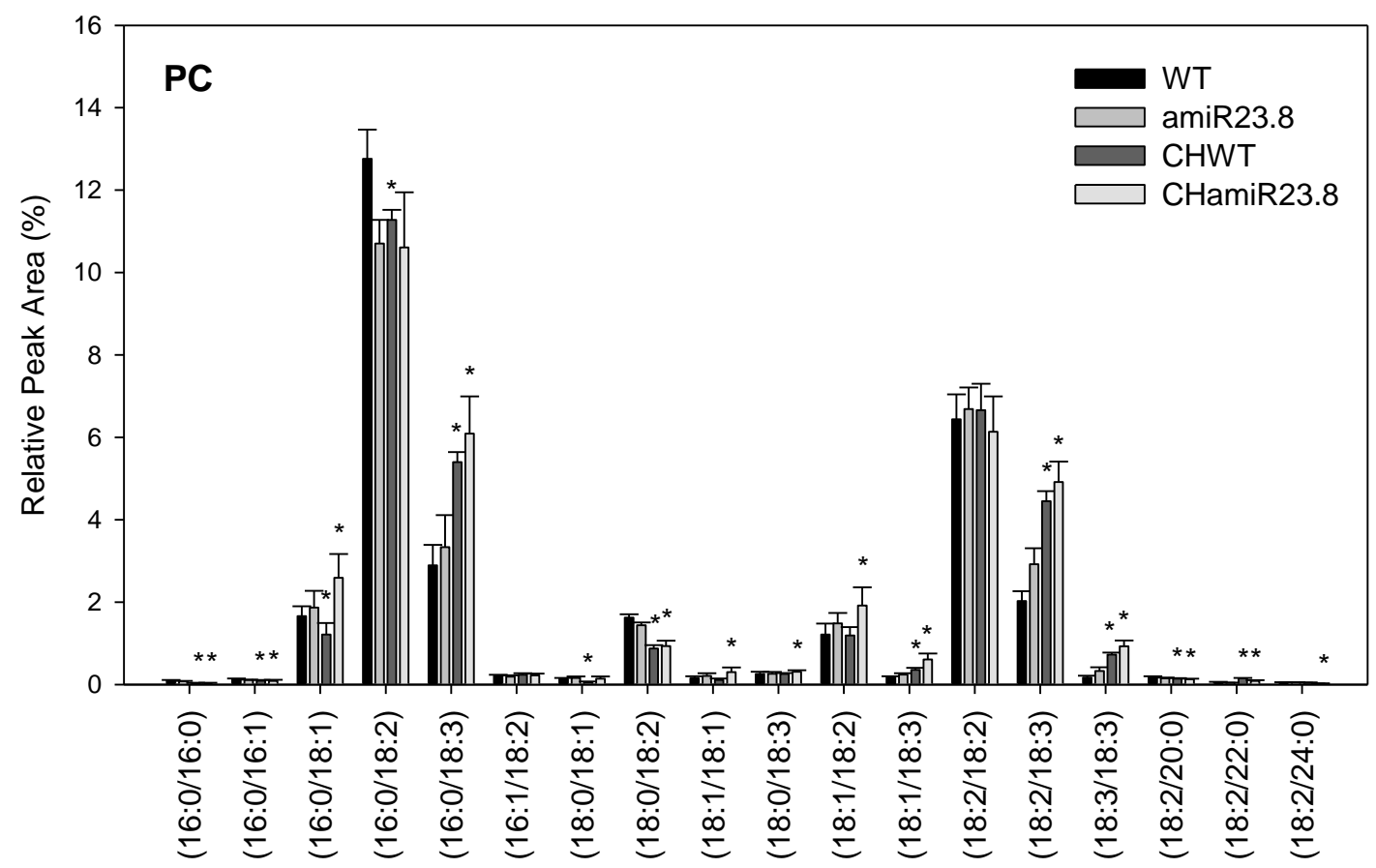

Supplemental figure 4.5. Lipid profile of phosphatidyl-choline (PC) in WT and amiR23.8 fruit. Only lipid species detected with a relative peak area $>0.05 \%$ were included in the dataset. Five independent amiR23.8 lines and two replicates per line were analyzed and the 10 samples were then combined to calculate mean and SD values. Six WT samples were also measured and taken as control. Samples consisted of a pool of three fruits from three individual plants. Asterisks indicate significance between normal and chilling conditions by one-way ANOVA, LSD Fisher test with $p<0.05$.

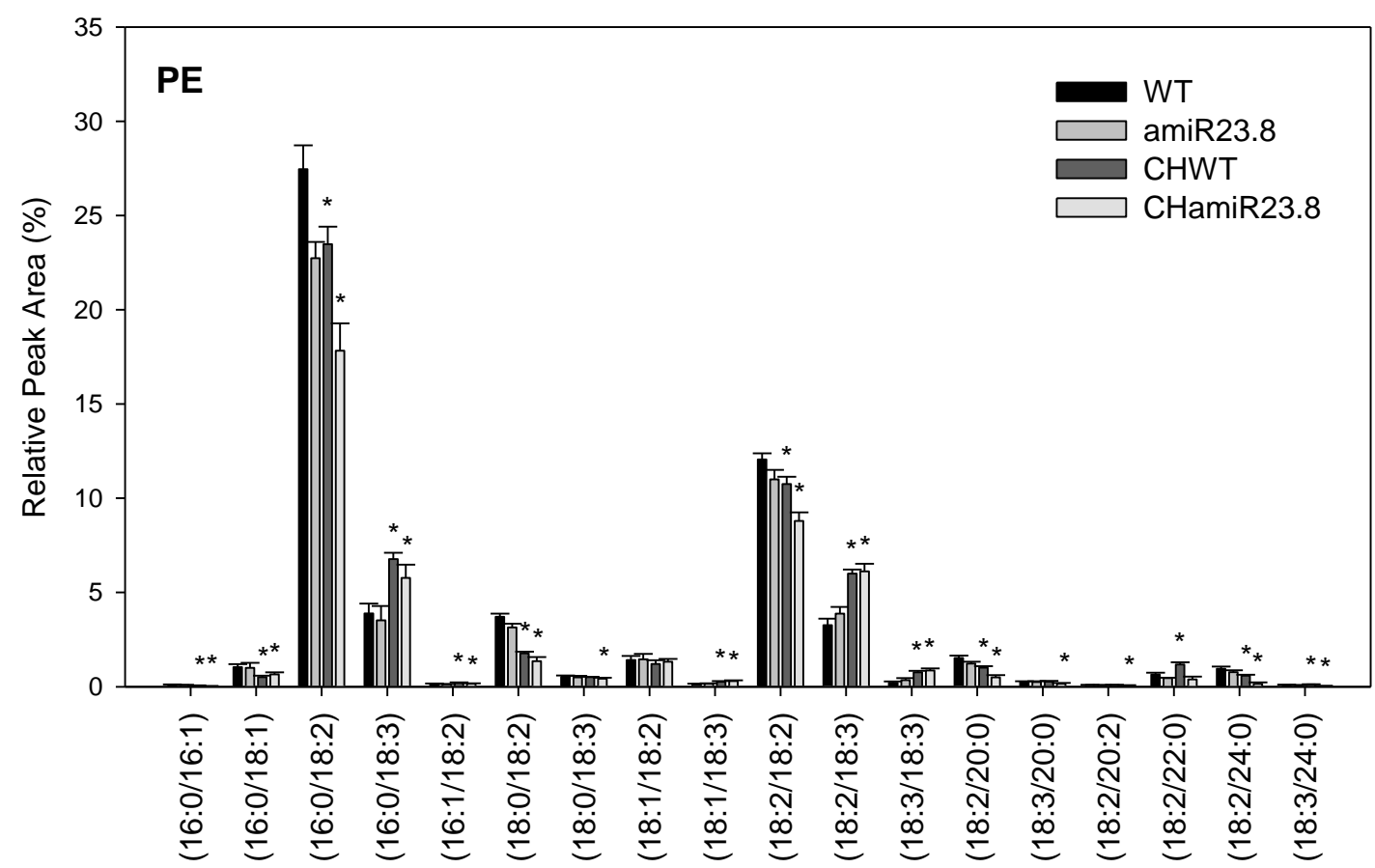

Supplemental figure 4.6. Lipid profile of phosphatidyl-ethanolamine (PE) in WT and amiR23.8 fruit. Only lipid species detected with a relative peak area $>0.05 \%$ were included in the dataset. Five independent amiR23.8 lines and two replicates per line were analyzed and the 10 samples were then combined to calculate mean and SD values. Six WT samples were also measured and taken as control. Samples consisted of a pool of three fruits from three individual plants. Asterisks indicate significance between normal and chilling conditions by one-way ANOVA, LSD Fisher test with $p<0.05$. 


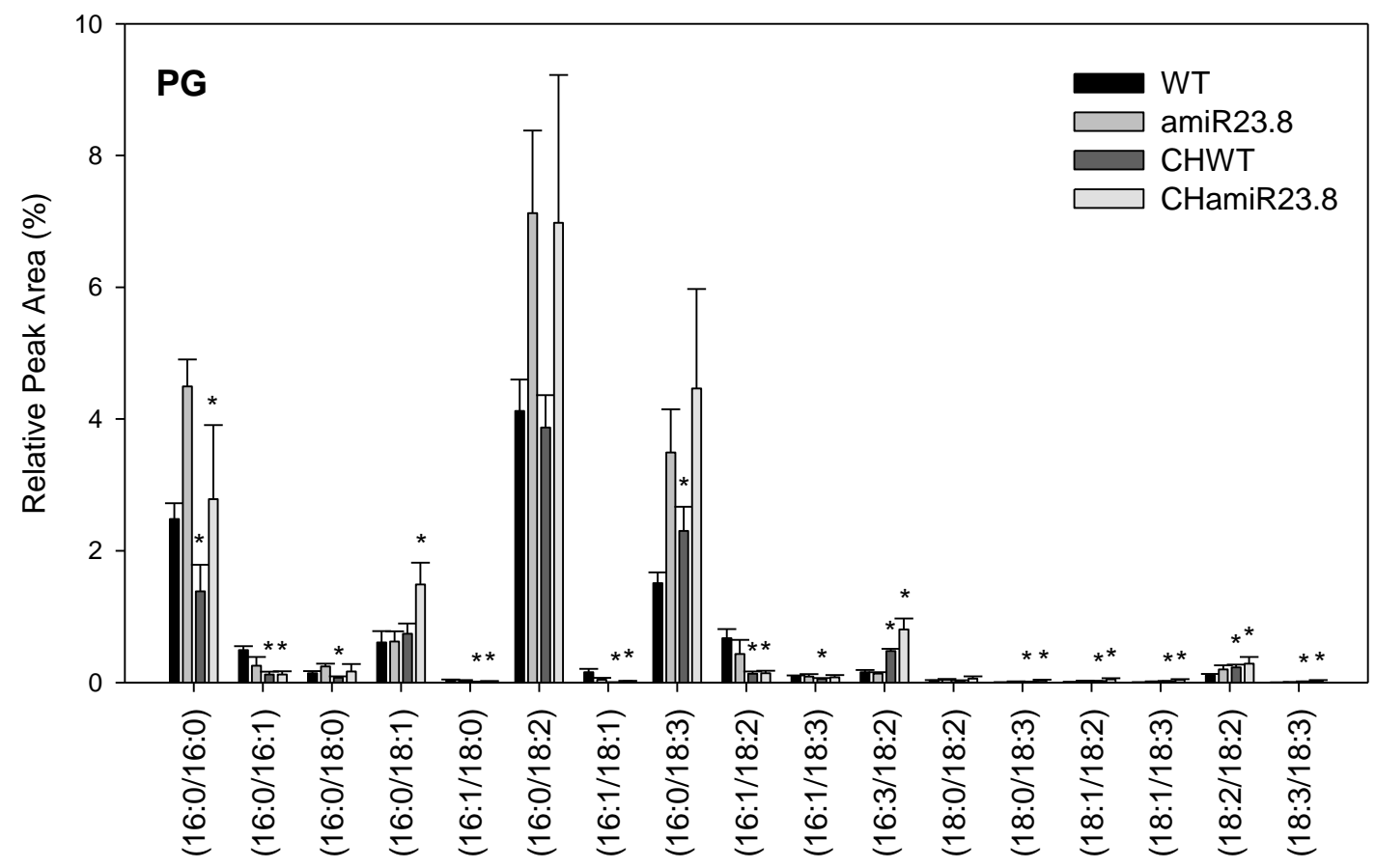

Supplemental figure 4.7. Lipid profile of phosphatidyl-glycerol (PG) in WT and amiR fruit. Only lipid species detected with a relative peak area $>0.02 \%$ were included in the dataset. Five independent amiR23.8 lines and two replicates per line were analyzed and the 10 samples were then combined to calculate mean and SD values. Six WT samples were also measured and taken as control. Samples consisted of a pool of three fruits from three individual plants. Asterisks indicate significance between normal and chilling conditions by one-way ANOVA, LSD Fisher test with $p<0.05$.

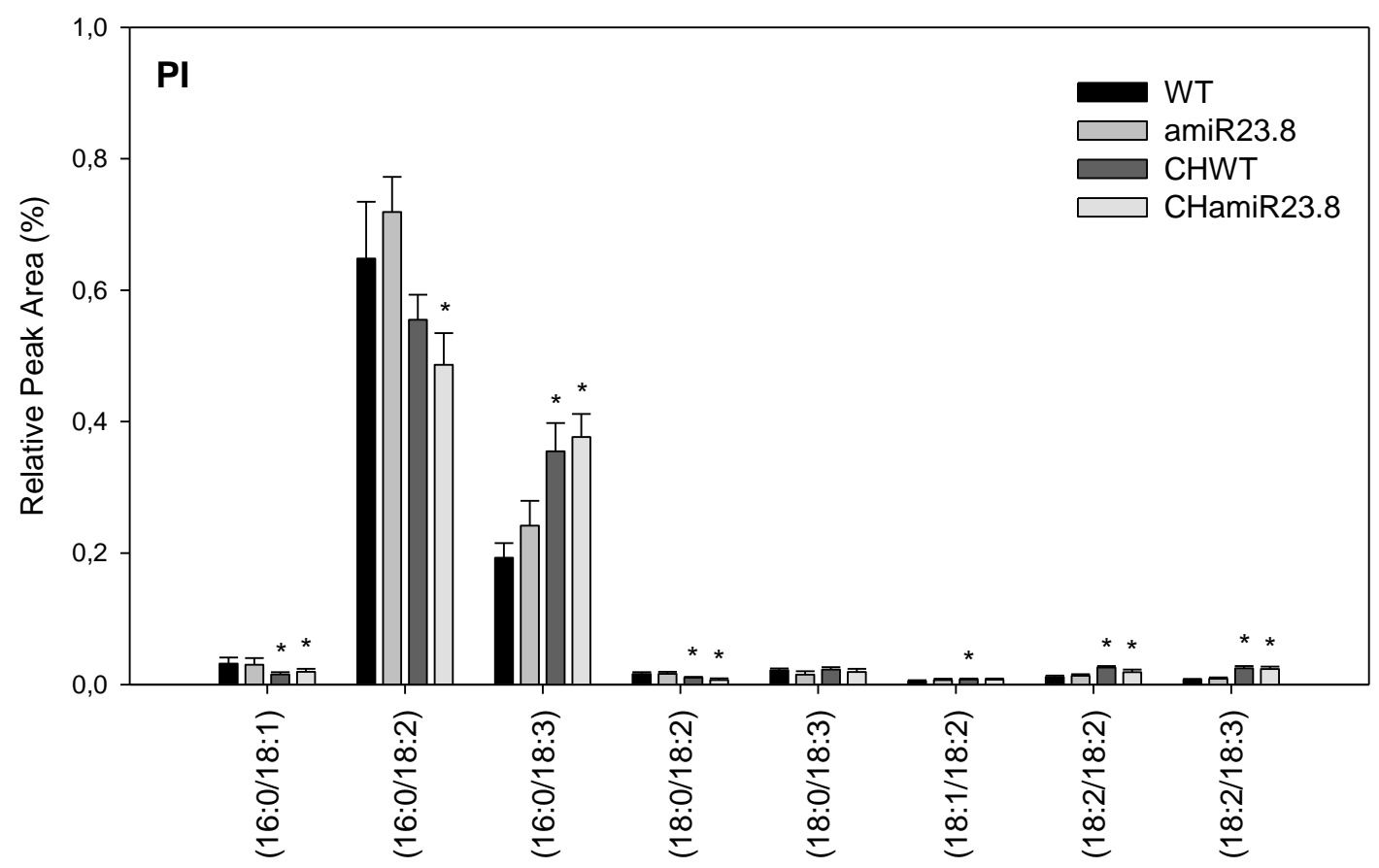

Supplemental figure 4.8. Lipid profile of phosphatidyl-inositol (PI) in WT and amiR fruit. Only lipid species detected with a relative peak area $>0.006 \%$ were included in the dataset. Five independent amiR23.8 lines and two replicates per line were analyzed and the 10 samples were then combined to calculate mean and SD values. Six WT samples were also measured and taken as control. Samples consisted of a pool of three fruits from three individual plants. Asterisks indicate significance between normal and chilling conditions by one-way ANOVA, LSD Fisher test with $p<0.05$. 


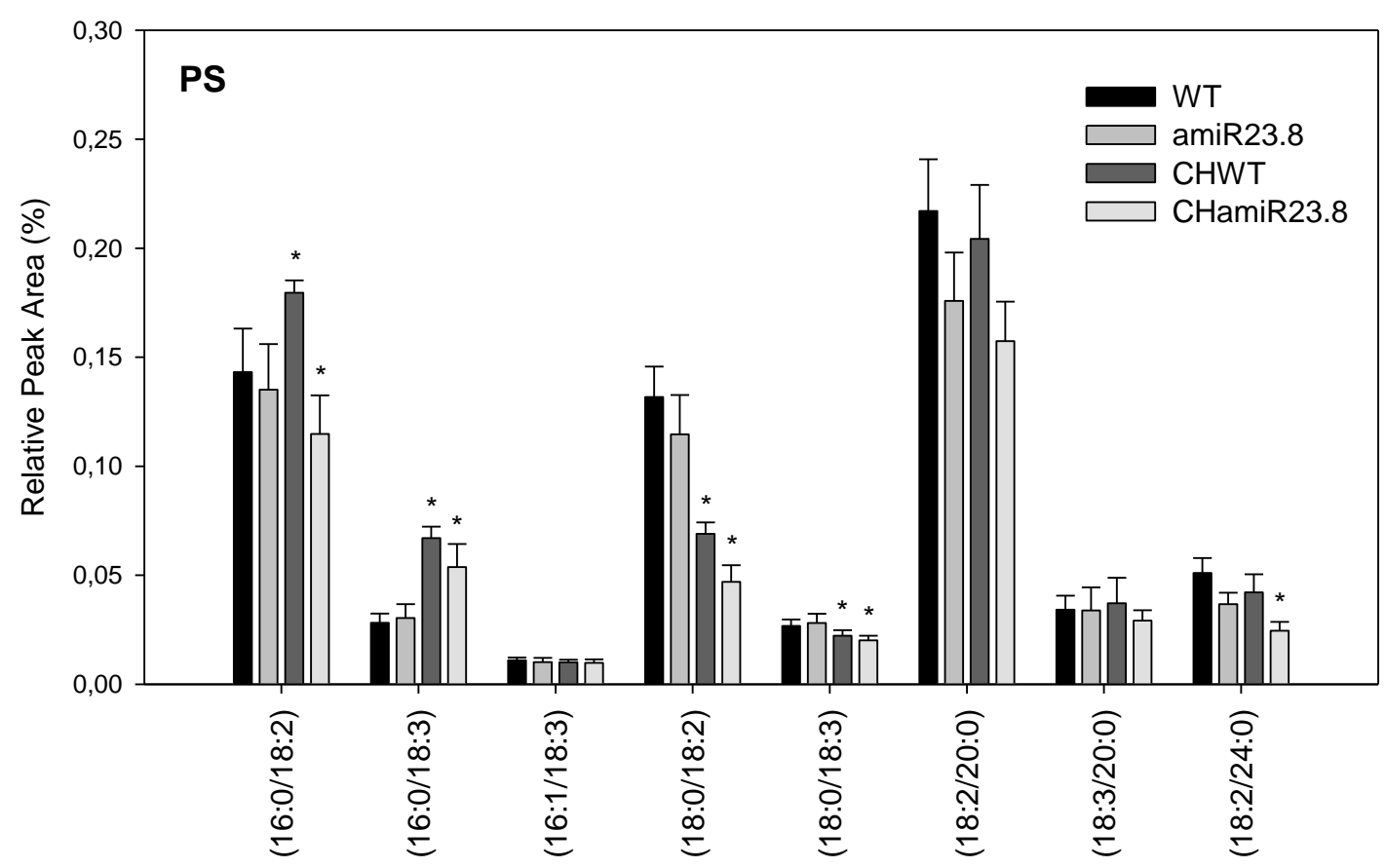

Supplemental figure 4.9. Lipid profile of phosphatidyl-serine (PS) in WT and amiR fruit. Only lipid species detected with a relative peak area $>0.01 \%$ were included in the dataset. Five independent amiR23.8 lines and two replicates per line were analyzed and the 10 samples were then combined to calculate mean and SD values. Six WT samples were also measured and taken as control. Samples consisted of a pool of three fruits from three individual plants. Asterisks indicate significance between normal and chilling conditions by one-way ANOVA, LSD Fisher test with $p<0.05$.

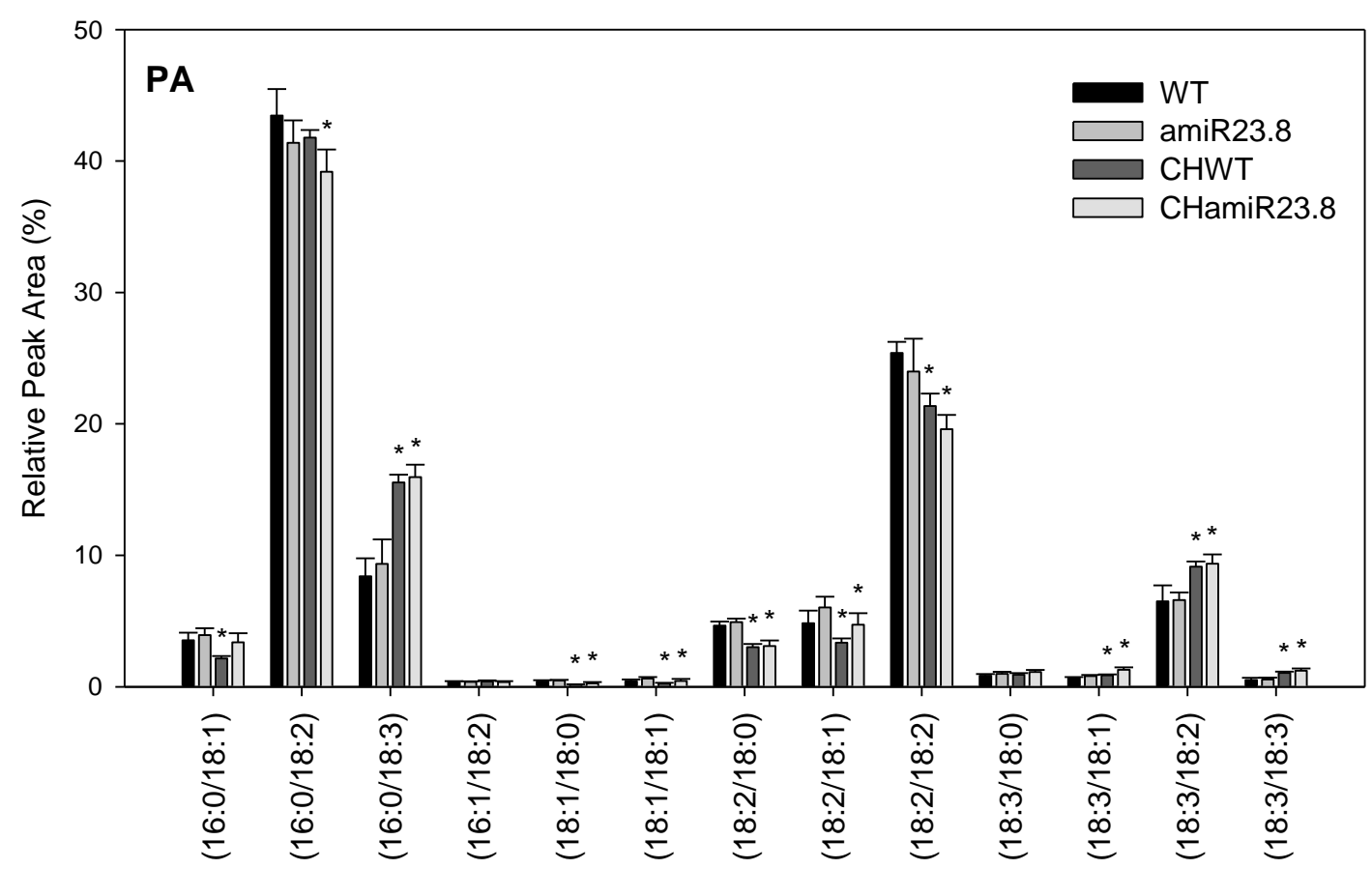

Supplemental figure 4.10. Lipid profile of phosphatidic acid (PA) in WT and amiR fruit. Only lipid species detected with a relative peak area $>0.3 \%$ were included in the dataset. Five independent amiR23.8 lines and two replicates per line were analyzed and the 10 samples were then combined to calculate mean and SD values. Six WT samples were also measured and taken as control. Samples consisted of a pool of three fruits from three individual plants. Asterisks indicate significance between normal and chilling conditions by one-way ANOVA, LSD Fisher test with $p<0.05$. 


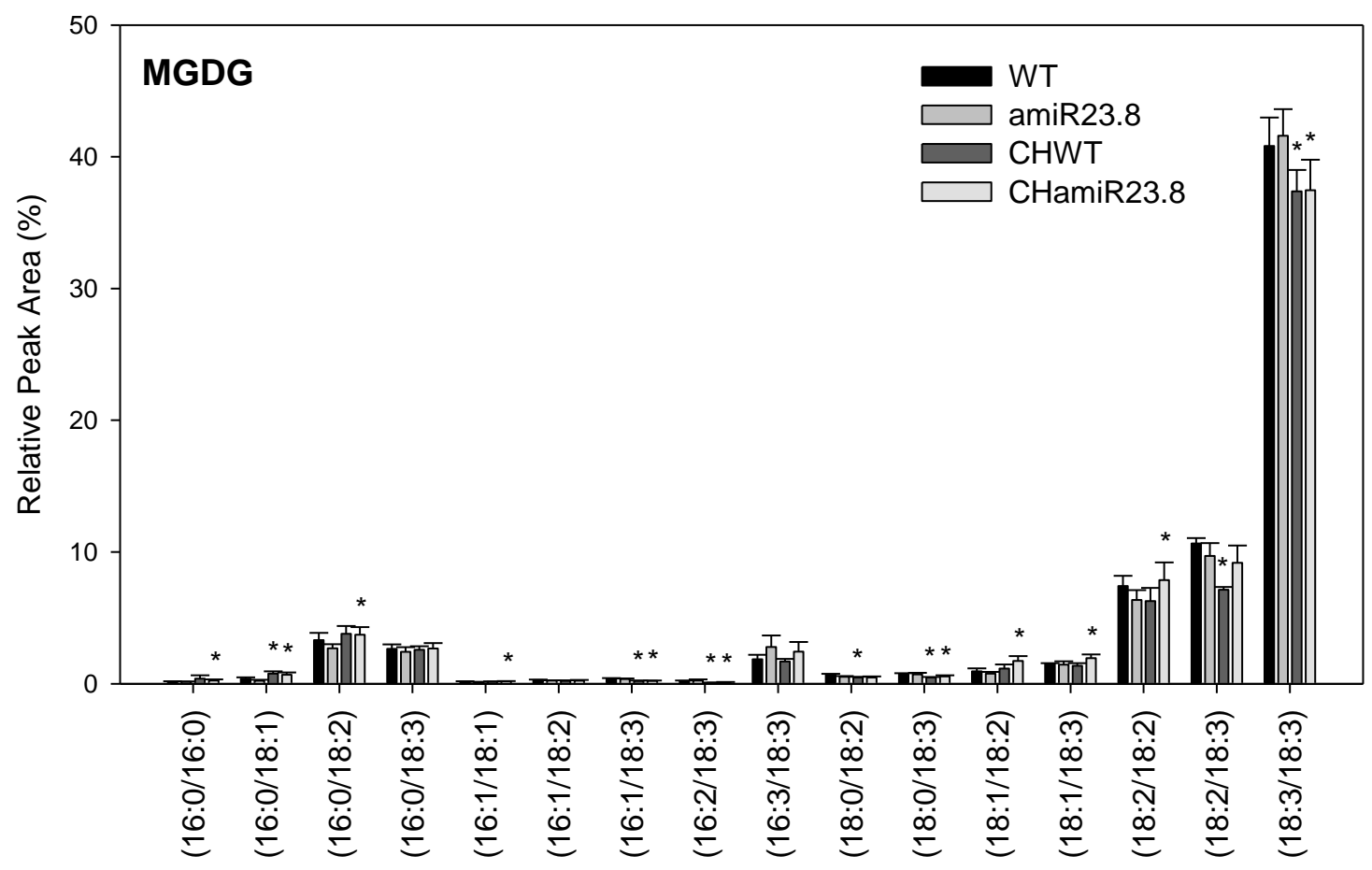

Supplemental figure 4.11. Lipid profile of monogalactosyldiacylglycerol (MGDG) in WT and amiR fruit. Only lipid species detected with a relative peak area $>0.1 \%$ were included in the dataset. Five independent amiR23.8 lines and two replicates per line were analyzed and the 10 samples were then combined to calculate mean and SD values. Six WT samples were also measured and taken as control. Samples consisted of a pool of three fruits from three individual plants. Asterisks indicate significance between normal and chilling conditions by one-way ANOVA, LSD Fisher test with $p<0.05$.

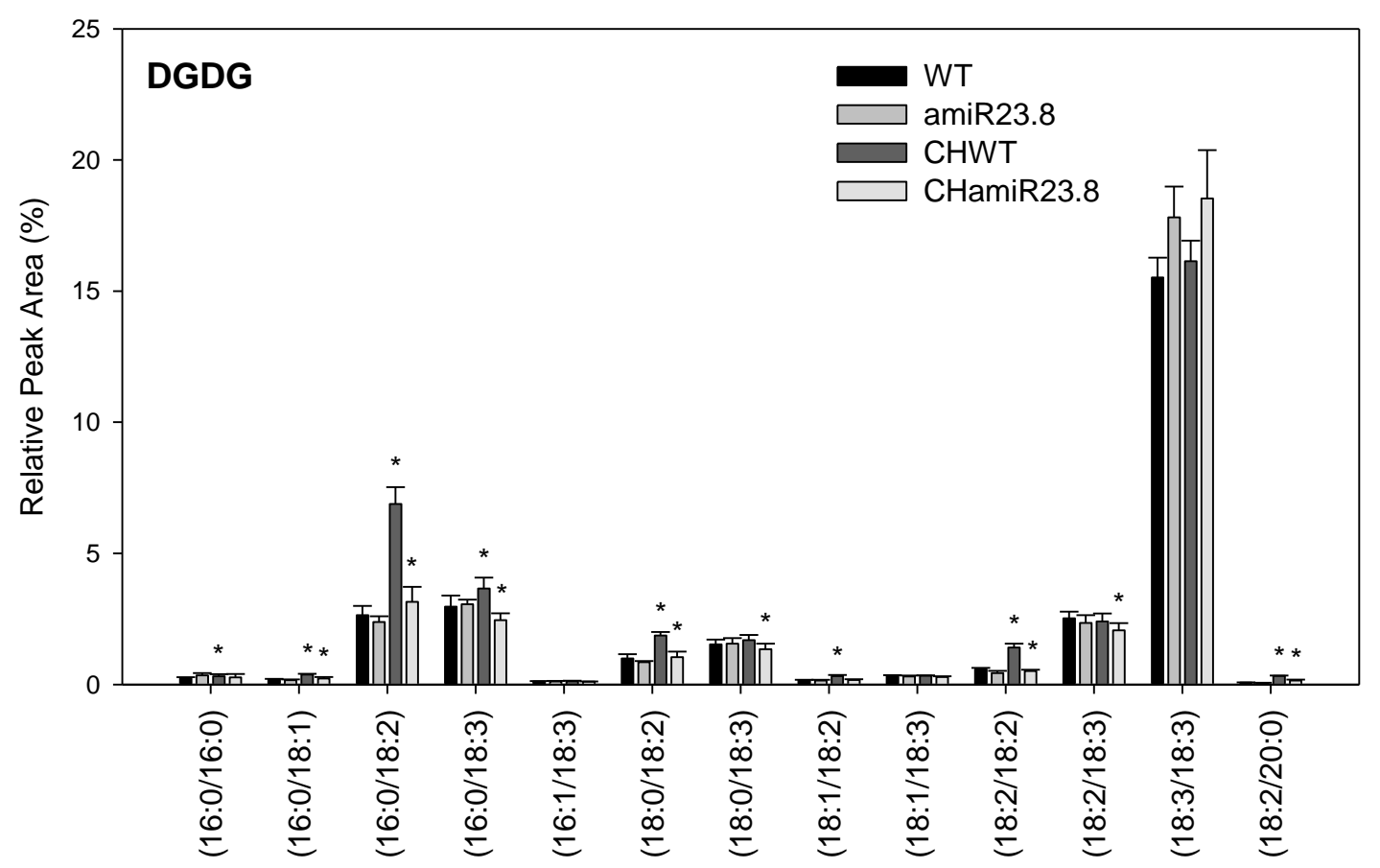

Supplemental figure 4.12. Lipid profile of digalactosyldiacylglycerol (DGDG) in WT and amiR fruit. Only lipid species detected with a relative peak area $>0.1 \%$ were included in the dataset. Five independent amiR23.8 lines and two replicates per line were analyzed and the 10 samples were then combined to calculate mean and SD values. Six WT samples were also measured and taken as control. Samples consisted of a pool of three fruits from three individual plants. Asterisks indicate significance between normal and chilling conditions by one-way ANOVA, LSD Fisher test with $p<0.05$. 


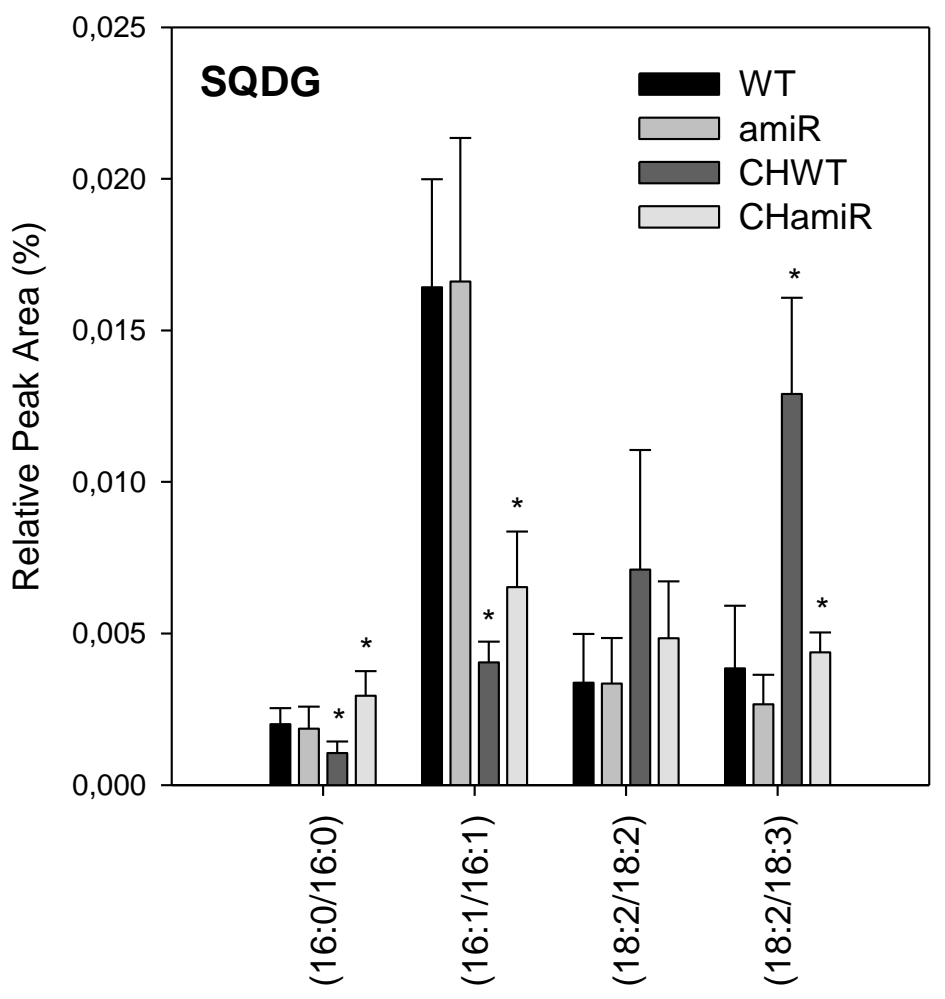

$\begin{array}{lrr}\text { Supplemental figure } & 4.13 . \\ \text { Lipid } & \text { profile } & \text { of }\end{array}$ sulfoquinovosyldiacylglycerol s (SQDG) in WT and amiR fruit. Only lipid species detected with a relative peak area $>0.001 \%$ were included in the dataset. Five independent amiR23.8 lines and two replicates per line were analyzed and the 10 samples were then combined to calculate mean and SD values. Six WT samples were also measured and taken as control. Samples consisted of a pool of three fruits from three individual plants. Asterisks indicate significance between normal and chilling conditions by one-way ANOVA, LSD Fisher test with $p<0.05$.

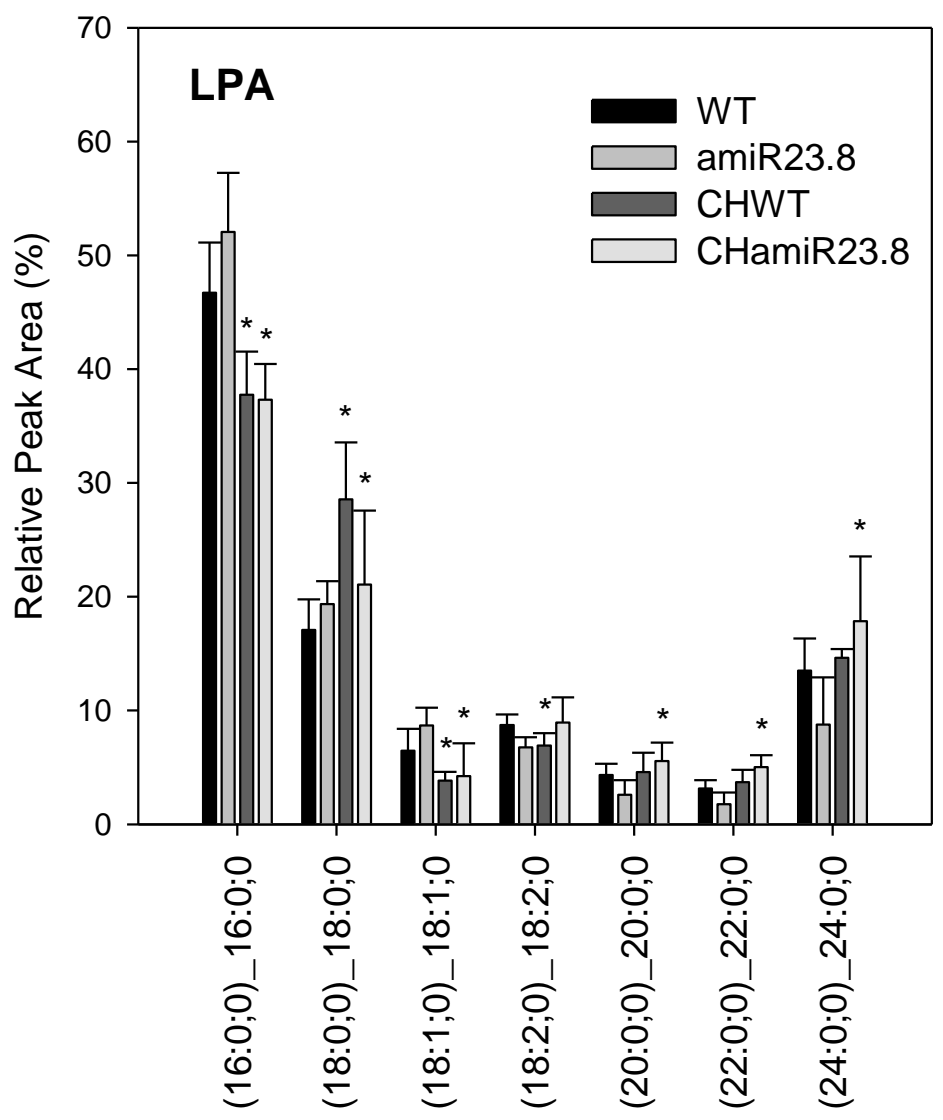

Supplemental figure 4.14. Lyso-PA composition in WT and amiR fruit. Only lipid species detected with a relative peak area $>3 \%$ were included in the dataset. Five independent amiR23.8 lines and two replicates per line were analyzed and the 10 samples were then combined to calculate mean and SD values. Six WT samples were also measured and taken as control. Samples consisted of a pool of three fruits from three individual plants. Asterisks indicate significance between normal and chilling conditions by one-way ANOVA, LSD Fisher test with $p<0.05$ 

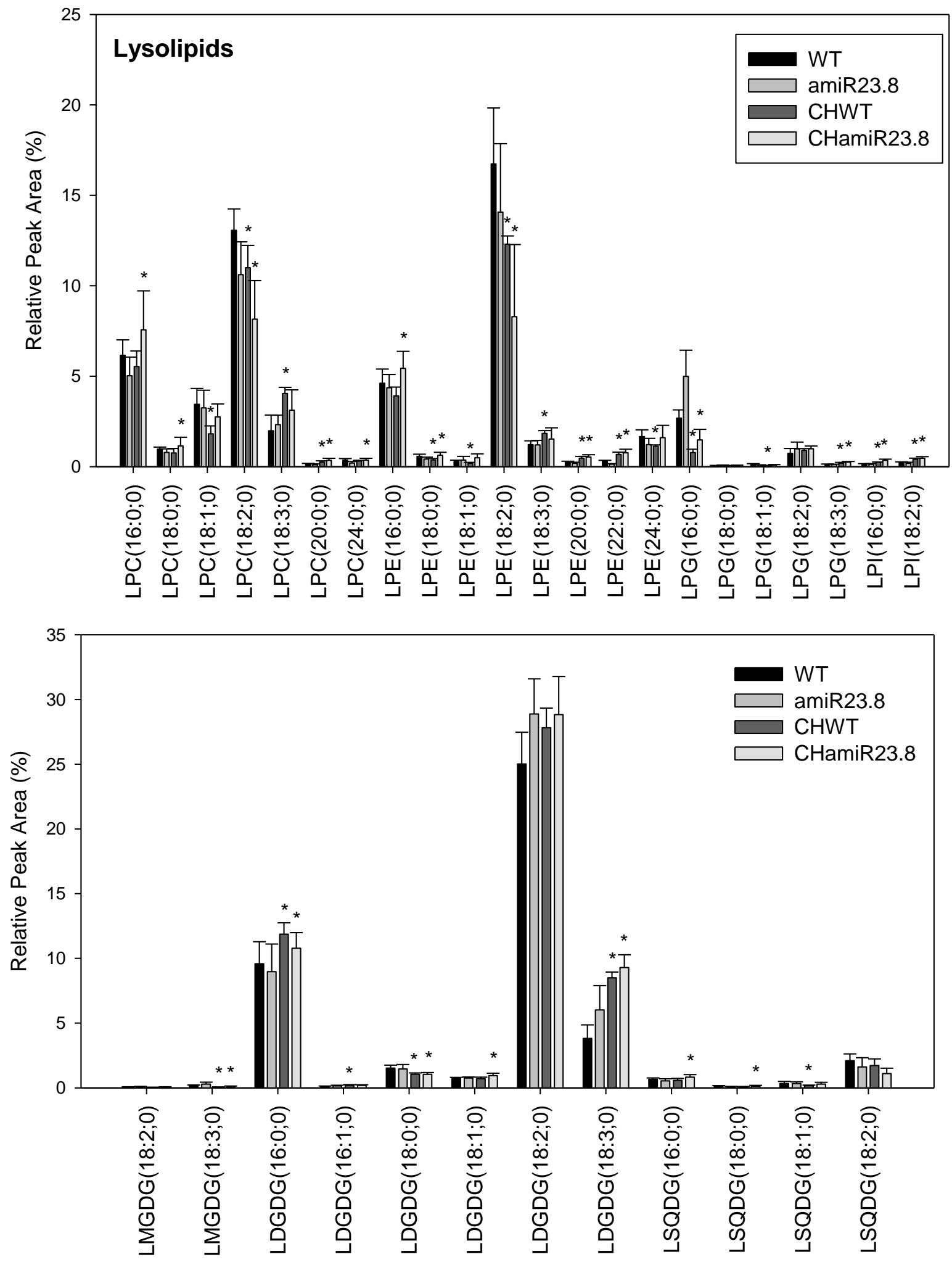

Supplemental figure 4.15. Lyso-lipid composition in WT and amiR fruit. Only lipid species detected with a relative peak area $>0.06 \%$ were included in the dataset. Five independent amiR23.8 lines and two replicates per line were analyzed and the 10 samples were then combined to calculate mean and SD values. Six WT samples were also measured and taken as control. Samples consisted of a pool of three fruits from three individual plants. Asterisks indicate significance between normal and chilling conditions by one-way ANOVA, LSD Fisher test with $p<0.05$. 


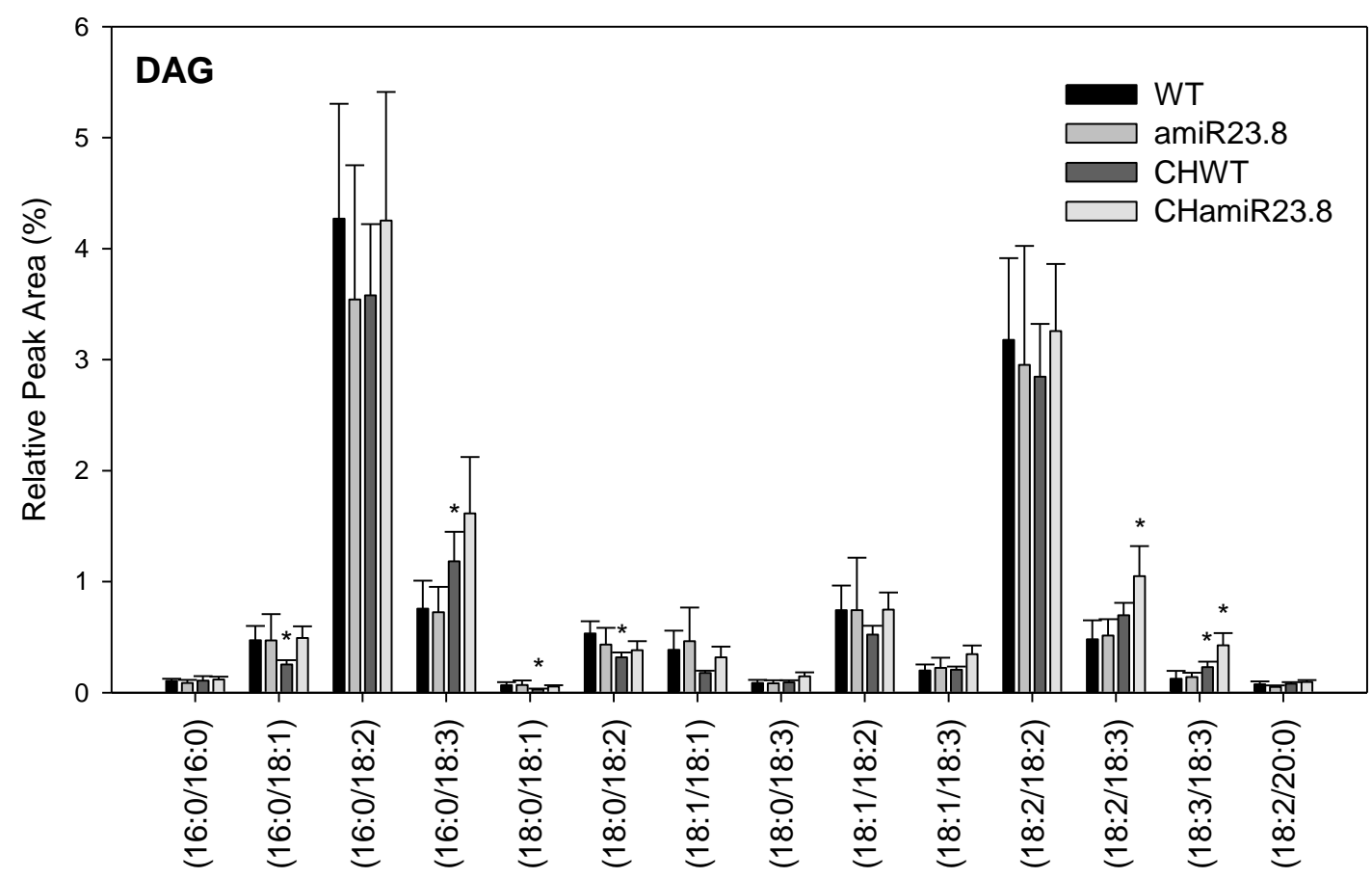

Supplemental figure 4.16. Lipid profile of diacylglycerides (DAG) in WT and amiR fruit. Only lipid species detected with a relative peak area $>0.08 \%$ were included in the dataset. Five independent amiR23.8 lines and two replicates per line were analyzed and the 10 samples were then combined to calculate mean and SD values. Six WT samples were also measured and taken as control. Samples consisted of a pool of three fruits from three individual plants. Asterisks indicate significance between normal and chilling conditions by one-way ANOVA, LSD Fisher test with $p<0.05$.

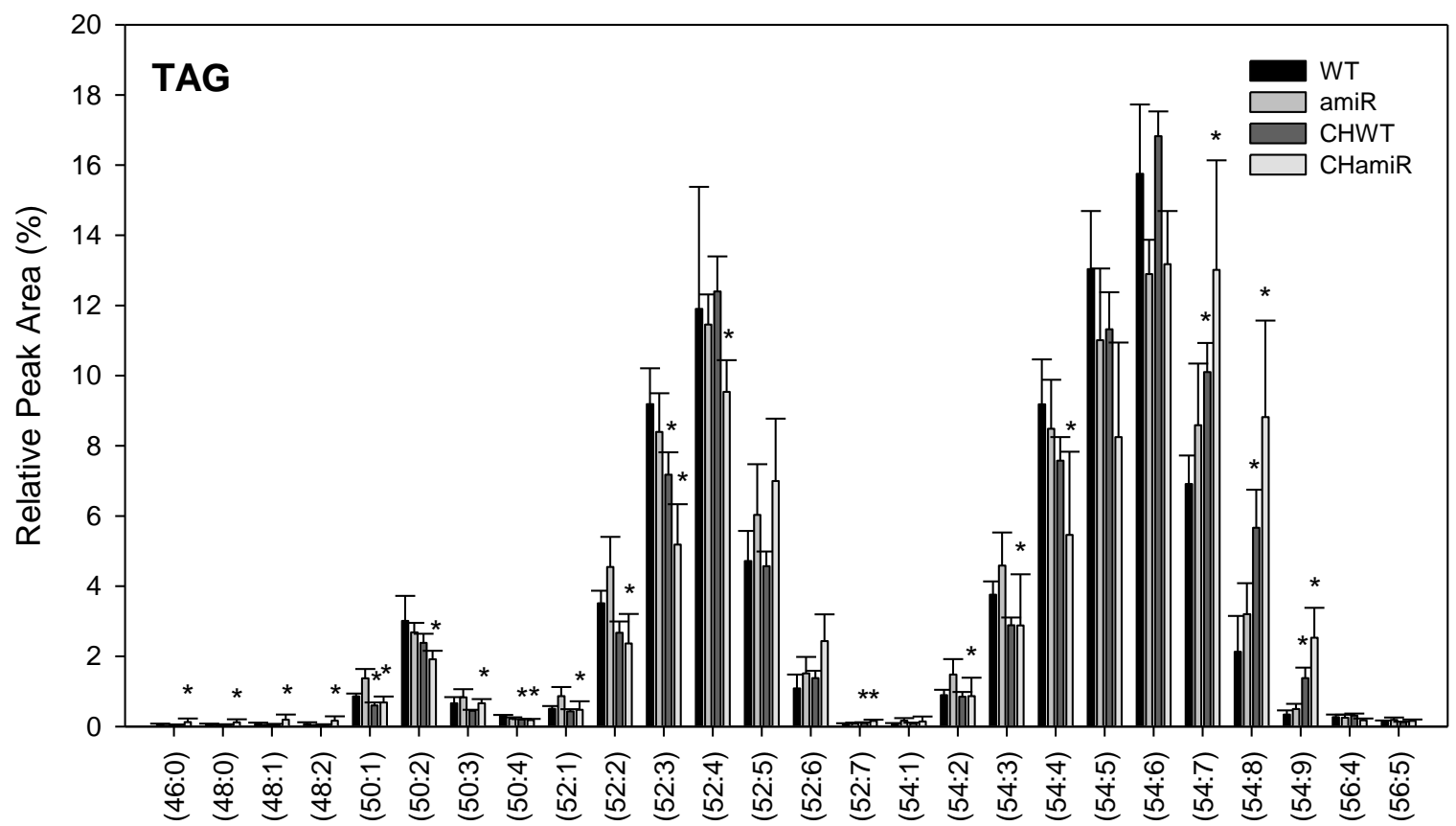

Supplemental figure 4.17. Lipid profile of triacylglycerides (TAG) in WT and amiR fruit. Only lipid species detected with a relative peak area $>0.5 \%$ were included in the dataset. Five independent amiR23.8 lines and two replicates per line were analyzed and the 10 samples were then combined to calculate mean and SD values. Six WT samples were also measured and taken as control. Samples consisted of a pool of three fruits from three individual plants. Asterisks indicate significance between normal and chilling conditions by one-way ANOVA, LSD Fisher test with $p<0.05$. 


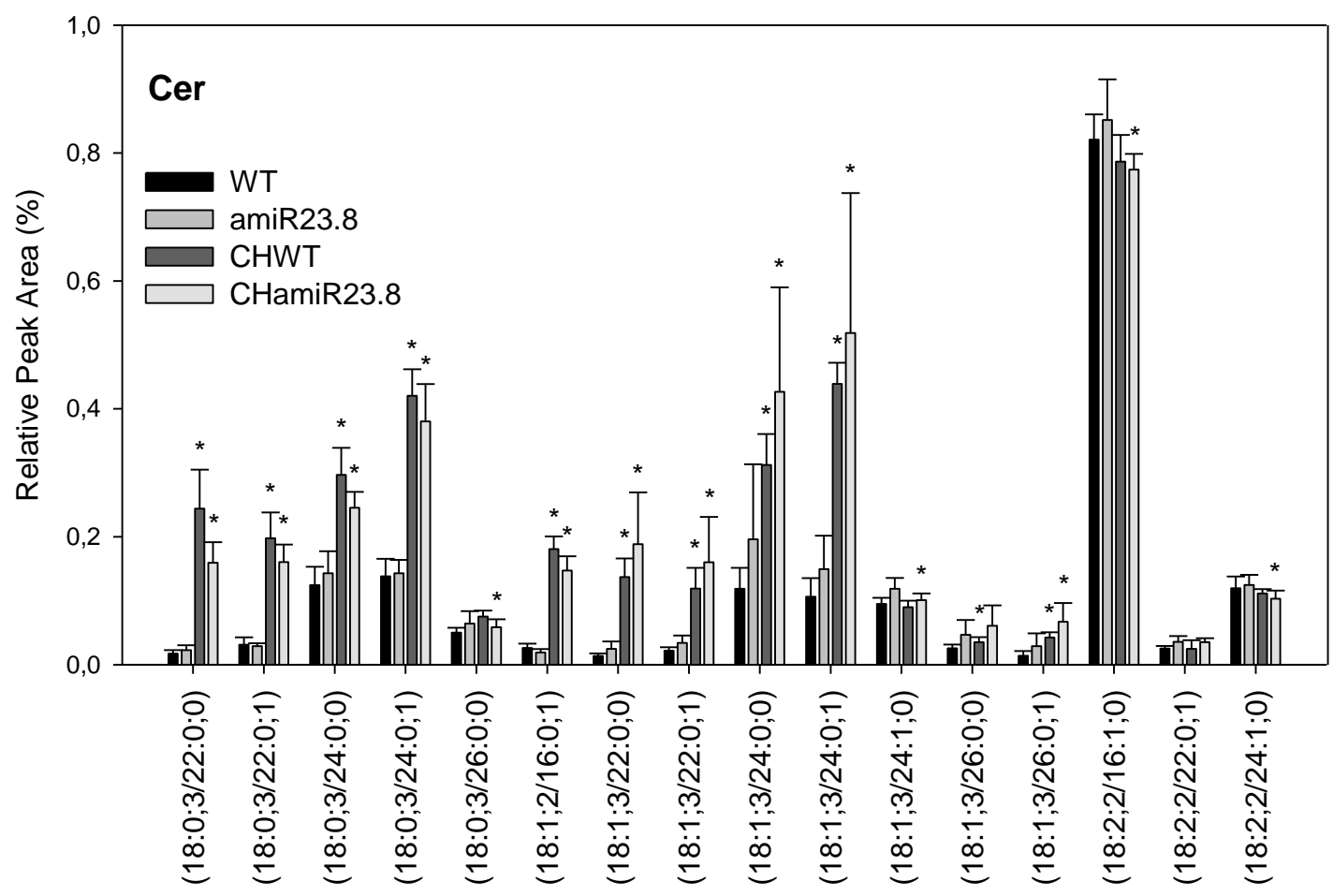

Supplemental figure 4.18. Lipid profile of ceramides (Cer) in WT and amiR fruit. Only lipid species detected with a relative peak area $>0.1 \%$ were included in the dataset. Five independent amiR23.8 lines and two replicates per line were analyzed and the 10 samples were then combined to calculate mean and SD values. Six WT samples were also measured and taken as control. Samples consisted of a pool of three fruits from three individual plants. Asterisks indicate significance between normal and chilling conditions by one-way ANOVA, LSD Fisher test with $p<0.05$.

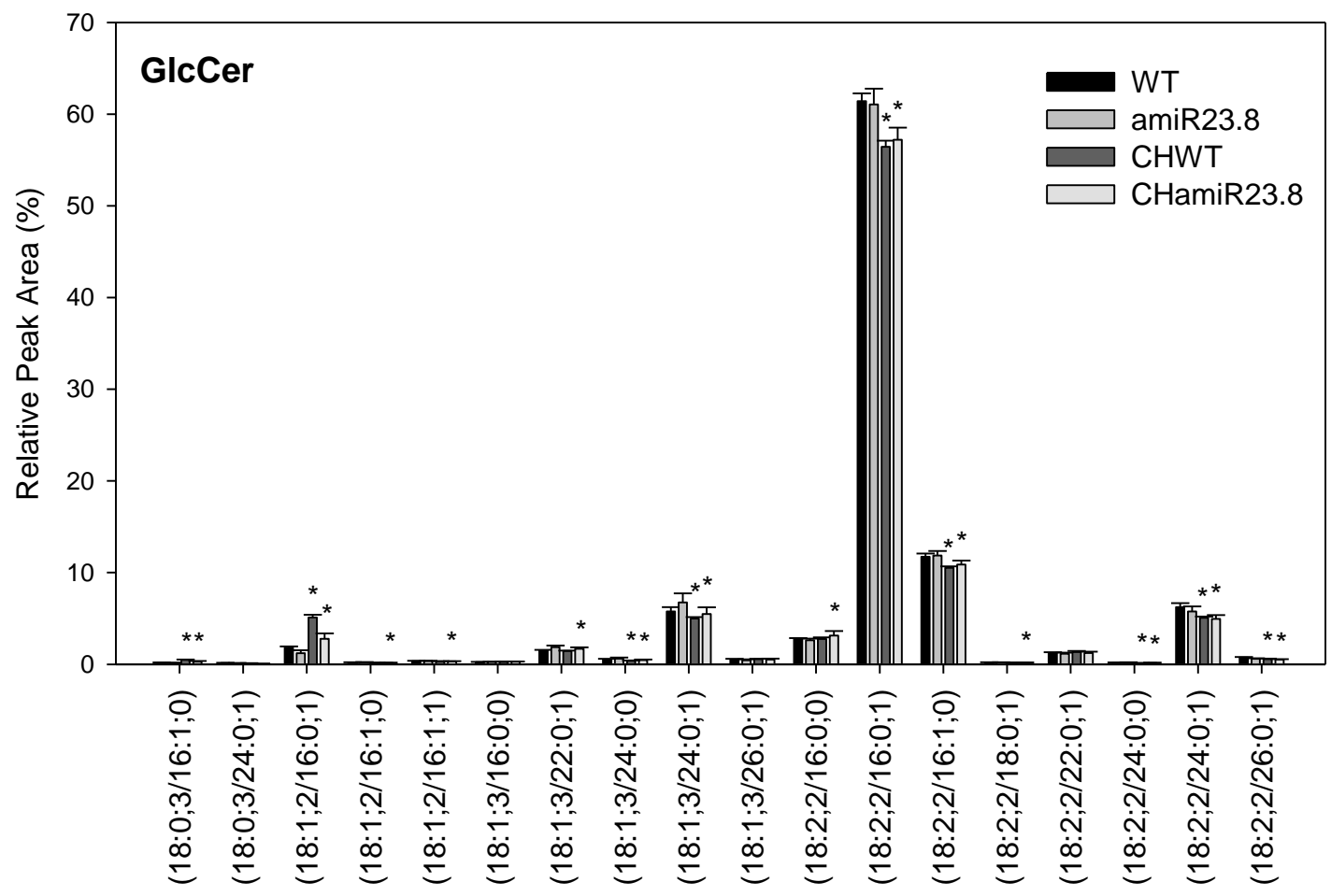

Supplemental figure 4.19. Lipid profile of glucosyl-ceramides (GlcCer) in WT and amiR fruit. Only lipid species detected with a relative peak area $>0.1 \%$ were included in the dataset. Five independent amiR23.8 lines and two replicates per line were analyzed and the 10 samples were then combined to calculate mean and SD values. Six WT samples were also measured and taken as control. Samples consisted of a pool of three fruits from three individual plants. Asterisks indicate significance between normal and chilling conditions by one-way ANOVA, LSD Fisher test with $p<0.05$. 


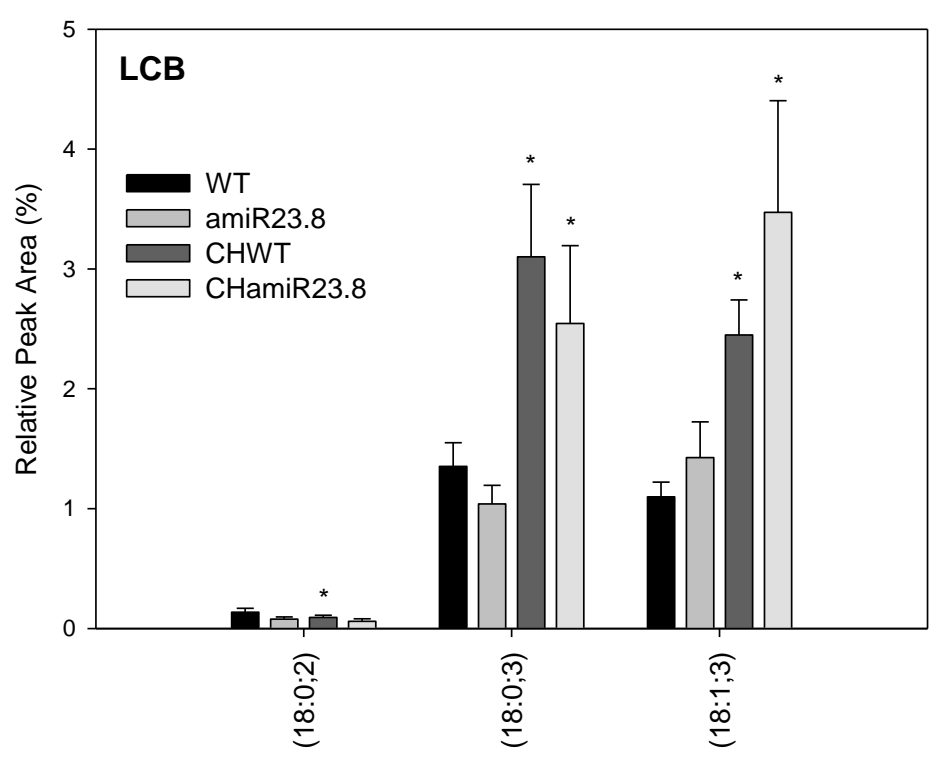

Supplemental figure 4.20. Lipid profile of (LCB) in WT and amiR fruit. Only lipid species detected with a relative peak area $>0.1 \%$ were included in the dataset. Five independent amiR23.8 lines and two replicates per line were analyzed and the 10 samples were then combined to calculate mean and SD values. Six WT samples were also measured and taken as control. Samples consisted of a pool of three fruits from three individual plants. Asterisks indicate significance between normal and chilling conditions by one-way ANOVA, LSD Fisher test with $p<0.05$.

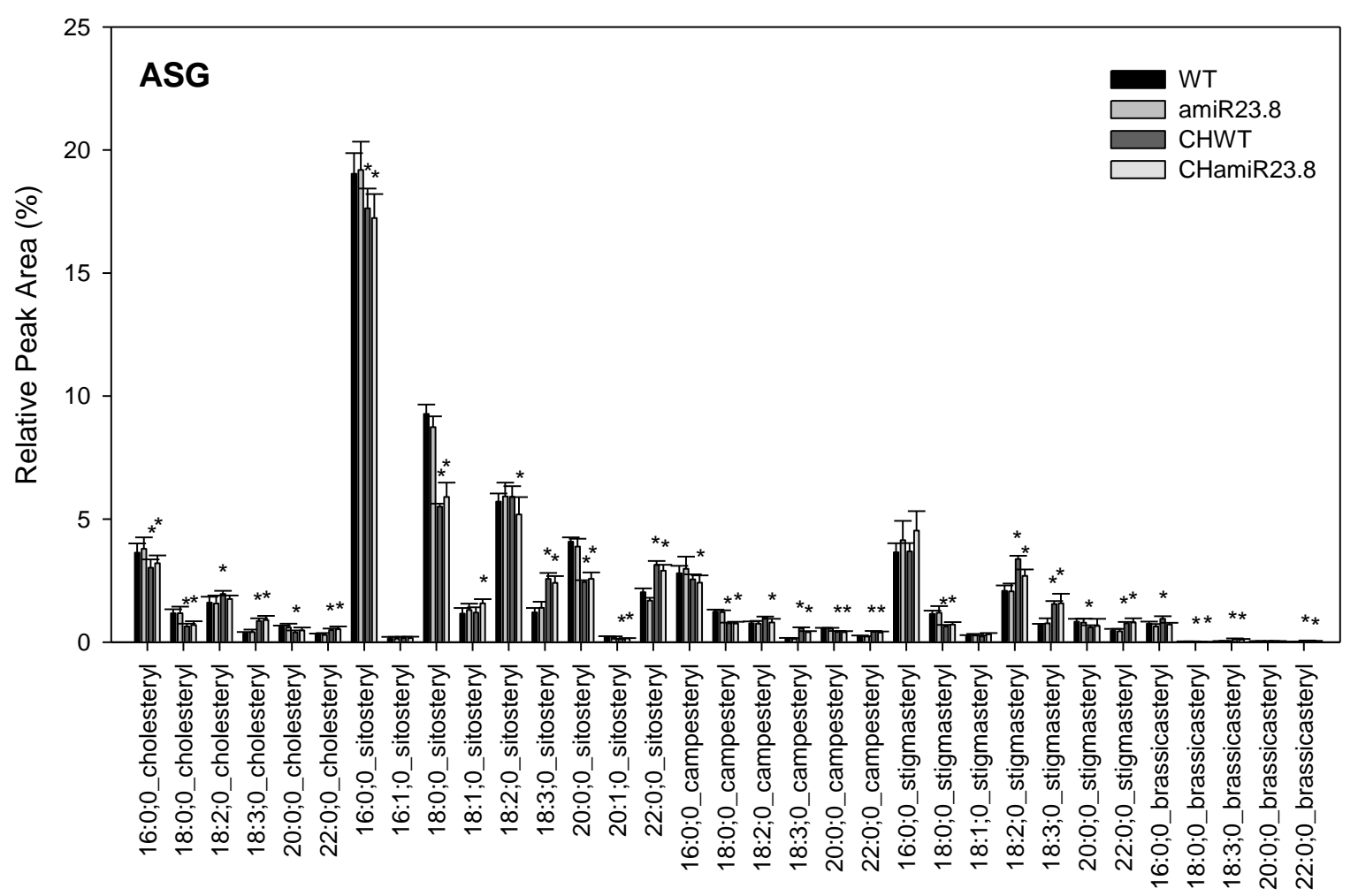

Supplemental figure 4.21. Lipid profile of acylsterylglucosides (ASG) in WT and amiR fruit. Only lipid species detected with a relative peak area $>0.02 \%$ were included in the dataset. Five independent amiR23.8 lines and two replicates per line were analyzed and the 10 samples were then combined to calculate mean and SD values. Six WT samples were also measured and taken as control. Samples consisted of a pool of three fruits from three individual plants. Asterisks indicate significance between normal and chilling conditions by one-way ANOVA, LSD Fisher test with $p<0.05$. 


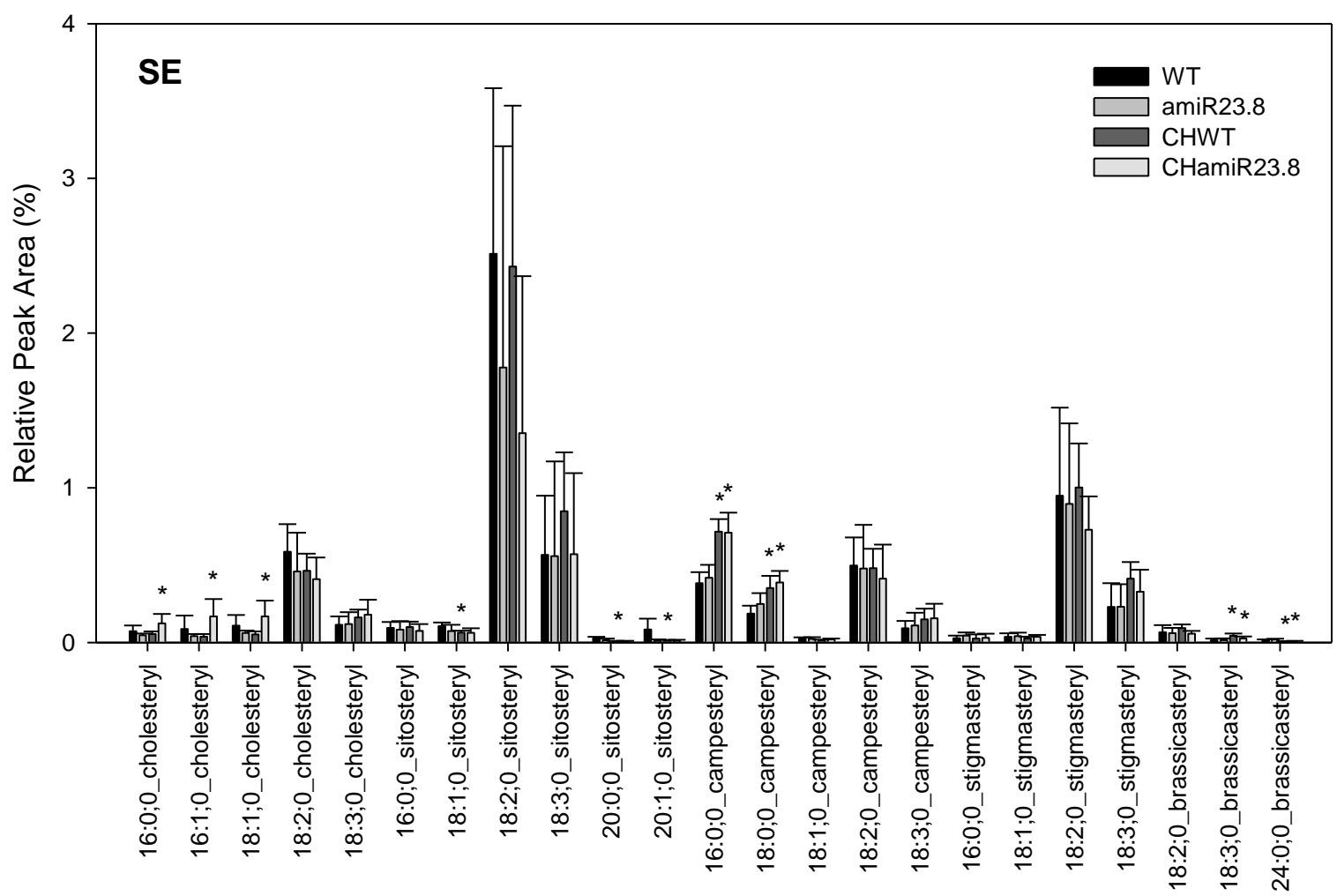

Supplemental figure 4.22. Lipid profile of sterolester (SE) in WT and amiR fruit. Only lipid species detected with a relative peak area $>0.01 \%$ were included in the dataset. Five independent amiR23.8 lines and two replicates per line were analyzed and the 10 samples were then combined to calculate mean and SD values. Six WT samples were also measured and taken as control. Samples consisted of a pool of three fruits from three individual plants. Asterisks indicate significance between normal and chilling conditions by one-way ANOVA, LSD Fisher test with $p<0.05$.

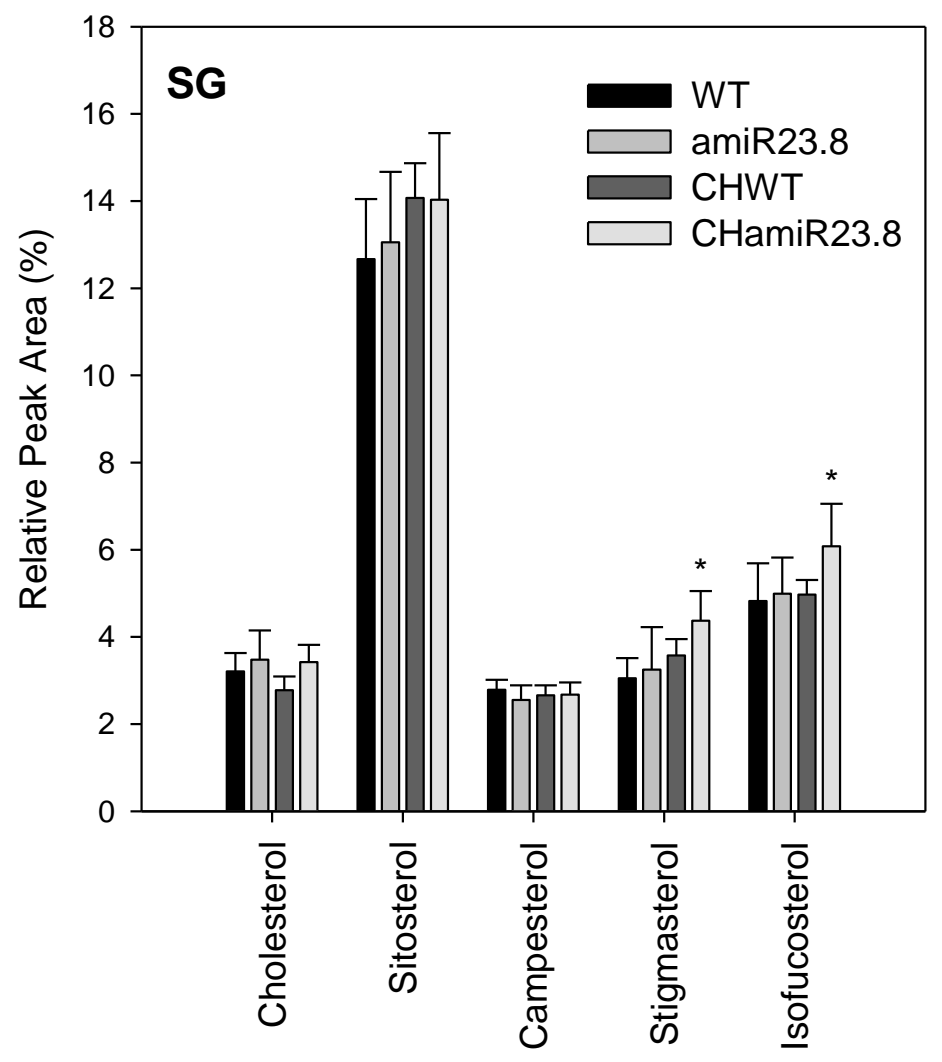

Supplemental figure 4.23. Lipid profile of sterylglucoside (SG) in WT and amiR fruit. Five independent amiR23.8 lines and two replicates per line were analyzed and the 10 samples were then combined to calculate mean and SD values. Six WT samples were also measured and taken as control. Samples consisted of a pool of three fruits from three individual plants. Asterisks indicate significance between normal and chilling conditions by one-way ANOVA, LSD Fisher test with $p<0.05$. 
Suppl. Table 4.1. List of proteins that are differentially expressed in amiR23.5 compared to control plants.

\begin{tabular}{|c|c|c|c|c|c|c|}
\hline Accession & Description & $\begin{array}{l}\text { Coverage } \\
{[\%]}\end{array}$ & $\begin{array}{l}\text { \# Unique } \\
\text { Peptides }\end{array}$ & $\begin{array}{l}\text { Fold } \\
\text { change }\end{array}$ & $\begin{array}{c}\text { Fold } \\
\text { change } \\
\left(\log _{2}\right)\end{array}$ & $\begin{array}{l}\text { Adj. P- } \\
\text { Value }\end{array}$ \\
\hline AT1G04510 & MOS4-associated complex $3 \mathrm{~A}$ & 11 & 2 & 100 & 6.64 & $6.4 \mathrm{E}-17$ \\
\hline AT3G09680 & Ribosomal protein S12/S23 family protein & 35 & 3 & 100 & 6.64 & $6.4 \mathrm{E}-17$ \\
\hline AT5G47190 & Ribosomal protein L19 family protein & 19 & 4 & 100 & 6.64 & $6.4 \mathrm{E}-17$ \\
\hline AT4G09650 & ATP synthase delta-subunit gene & 15 & 3 & 100 & 6.64 & $6.4 \mathrm{E}-17$ \\
\hline AT1G21720 & proteasome beta subunit $\mathrm{C} 1$ & 25 & 3 & 100 & 6.64 & $6.4 \mathrm{E}-17$ \\
\hline AT1G74970 & ribosomal protein S9 & 31 & 6 & 100 & 6.64 & $6.4 \mathrm{E}-17$ \\
\hline AT5G39730 & AIG2-like (avirulence induced gene) family protein & 28 & 4 & 100 & 6.64 & $6.4 \mathrm{E}-17$ \\
\hline AT2G22170 & Lipase/lipooxygenase, PLAT/LH2 family protein & 21 & 4 & 100 & 6.64 & $6.4 \mathrm{E}-17$ \\
\hline AT1G11750 & CLP protease proteolytic subunit 6 & 31 & 5 & 100 & 6.64 & $6.4 \mathrm{E}-17$ \\
\hline AT5G27390 & $\begin{array}{l}\text { Mog1/PsbP/DUF1795-like photosystem II reaction center PsbP family } \\
\text { protein }\end{array}$ & 16 & 4 & 100 & 6.64 & $6.4 \mathrm{E}-17$ \\
\hline AT3G08740 & elongation factor $\mathrm{P}(\mathrm{EF}-\mathrm{P})$ family protein & 38 & 9 & 100 & 6.64 & $6.4 \mathrm{E}-17$ \\
\hline AT5G18420 & unknown protein & 5 & 2 & 100 & 6.64 & $6.4 \mathrm{E}-17$ \\
\hline AT3G47810 & Calcineurin-like metallo-phosphoesterase superfamily prot & 17 & 3 & 100 & 6.64 & $6.4 \mathrm{E}-17$ \\
\hline AT3G46000 & actin depolymerizing factor 2 & 24 & 2 & 100 & 6.64 & $6.4 \mathrm{E}-17$ \\
\hline AT2G19740 & Ribosomal protein L31e family protein & 8 & 2 & 100 & 6.64 & $6.4 \mathrm{E}-17$ \\
\hline AT5G56260 & $\begin{array}{l}\text { Ribonuclease E inhibitor RraA/Dimethylmenaquinone } \\
\text { methyltransferase }\end{array}$ & 13 & 2 & 100 & 6.64 & $6.4 \mathrm{E}-17$ \\
\hline AT2G06050 & oxophytodienoate-reductase 3 & 6 & 2 & 100 & 6.64 & $6.4 \mathrm{E}-17$ \\
\hline AT5G23010 & methylthioalkylmalate synthase 1 & 7 & 3 & 100 & 6.64 & $6.4 \mathrm{E}-17$ \\
\hline AT1G63970 & isoprenoid $\mathrm{F}$ & 17 & 3 & 100 & 6.64 & $6.4 \mathrm{E}-17$ \\
\hline AT5G61970 & signal recognition particle-related / SRP-related & 6 & 2 & 100 & 6.64 & $6.4 \mathrm{E}-17$ \\
\hline AT5G64040 & $\begin{array}{l}\text { photosystem I reaction center subunit PSI-N, chloroplast, putative / } \\
\text { PSI-N, putative (PSAN) }\end{array}$ & 11 & 2 & 100 & 6.64 & $6.4 \mathrm{E}-17$ \\
\hline AT3G21220 & MAP kinase kinase 5 & 4 & 2 & 100 & 6.64 & $6.4 \mathrm{E}-17$ \\
\hline AT5G48760 & Ribosomal protein L13 family protein & 34 & 2 & 100 & 6.64 & $6.4 \mathrm{E}-17$ \\
\hline AT3G05590 & ribosomal protein L18 & 52 & 3 & 100 & 6.64 & $6.4 \mathrm{E}-17$ \\
\hline AT1G53670 & methionine sulfoxide reductase B 1 & 10 & 2 & 100 & 6.64 & $6.4 \mathrm{E}-17$ \\
\hline AT3G46630 & Protein of unknown function (DUF3223) & 9 & 2 & 100 & 6.64 & $6.4 \mathrm{E}-17$ \\
\hline AT1G24020 & MLP-like protein 423 & 36 & 6 & 100 & 6.64 & $6.4 \mathrm{E}-17$ \\
\hline AT5G28050 & Cytidine/deoxycytidylate deaminase family protein & 21 & 3 & 100 & 6.64 & $6.4 \mathrm{E}-17$ \\
\hline AT4G34870 & rotamase cyclophilin 5 & 42 & 5 & 41.32 & 5.37 & $6.4 \mathrm{E}-17$ \\
\hline AT5G38430 & Ribulose bisphosphate carboxylase family protein & 69 & 7 & 30.00 & 4.91 & $6.4 \mathrm{E}-17$ \\
\hline AT1G22840 & CYTOCHROME C-1 & 17 & 2 & 8.31 & 3.05 & 1.2E-11 \\
\hline AT1G48350 & Ribosomal L18p/L5e family protein & 25 & 4 & 7.62 & 2.93 & $2.6 \mathrm{E}-13$ \\
\hline AT2G19760 & profilin 1 & 43 & 4 & 7.33 & 2.87 & 1.7E-09 \\
\hline AT5G65350 & histone 311 & 42 & 2 & 6.72 & 2.75 & $1.8 \mathrm{E}-11$ \\
\hline AT4G12800 & photosystem I subunit I & 19 & 3 & 6.37 & 2.67 & 4.7E-09 \\
\hline AT1G14410 & ssDNA-binding transcriptional regulator & 21 & 3 & 5.74 & 2.52 & 5.7E-07 \\
\hline AT1G62290 & Saposin-like aspartyl protease family protein & 16 & 3 & 5.07 & 2.34 & 1.6E-07 \\
\hline AT1G54780 & thylakoid lumen $18.3 \mathrm{kDa}$ protein & 20 & 5 & 4.61 & 2.21 & $3.4 \mathrm{E}-07$ \\
\hline AT3G55330 & PsbP-like protein 1 & 43 & 8 & 3.91 & 1.97 & $1.2 \mathrm{E}-09$ \\
\hline AT3G45140 & lipoxygenase 2 & 59 & 39 & 3.86 & 1.95 & $2.8 \mathrm{E}-13$ \\
\hline AT3G63540 & $\begin{array}{l}\text { Mog1/PsbP/DUF1795-like photosystem II reaction center PsbP family } \\
\text { protein }\end{array}$ & 50 & 5 & 3.25 & 1.7 & 1.1E-04 \\
\hline AT2G25070 & Protein phosphatase $2 \mathrm{C}$ family protein & 5 & 2 & 3.22 & 1.69 & 4.8E-03 \\
\hline AT5G14670 & ADP-ribosylation factor A1B & 63 & 13 & 3.19 & 1.67 & 9.6E-09 \\
\hline AT3G14210 & epithiospecifier modifier 1 & 47 & 13 & 3.13 & 1.65 & $3.3 \mathrm{E}-10$ \\
\hline AT5G25980 & glucoside glucohydrolase 2 & 42 & 17 & 3.00 & 1.59 & 6.3E-09 \\
\hline AT5G20630 & germin 3 & 64 & 7 & 2.98 & 1.57 & $6.2 \mathrm{E}-07$ \\
\hline AT3G54600 & Class I glutamine amidotransferase-like superfamily protein & 8 & 2 & 2.68 & 1.42 & $1.0 \mathrm{E}-02$ \\
\hline AT5G51110 & Transcriptional coactivator/pterin dehydratase & 12 & 2 & 2.54 & 1.35 & 2.1E-02 \\
\hline AT4G30140 & GDSL-like Lipase/Acylhydrolase superfamily protein & 10 & 3 & 2.39 & 1.26 & $1.1 \mathrm{E}-02$ \\
\hline AT1G52400 & beta glucosidase 18 & 22 & 5 & 2.37 & 1.24 & $2.0 \mathrm{E}-03$ \\
\hline AT4G36700 & RmIC-like cupins superfamily protein & 16 & 6 & 2.27 & 1.18 & $1.5 \mathrm{E}-03$ \\
\hline AT5G23900 & Ribosomal protein $\mathrm{L} 13 \mathrm{e}$ family protein & 33 & 2 & 0.18 & -2.45 & $4.8 \mathrm{E}-06$ \\
\hline AT4G23570 & phosphatase-related & 11 & 3 & 0.01 & -6.64 & $6.4 \mathrm{E}-17$ \\
\hline AT2G01720 & Ribophorin I & 8 & 2 & 0.01 & -6.64 & $6.4 \mathrm{E}-17$ \\
\hline AT1G62480 & Vacuolar calcium-binding protein-related & 38 & 2 & 0.01 & -6.64 & $6.4 \mathrm{E}-17$ \\
\hline AT5G34940 & glucuronidase 3 & 5 & 2 & 0.01 & -6.64 & $6.4 \mathrm{E}-17$ \\
\hline AT3G10090 & Nucleic acid-binding, OB-fold-like protein & 23 & 2 & 0.01 & -6.64 & $6.4 \mathrm{E}-17$ \\
\hline
\end{tabular}

Suppl. Table 4.2. List of proteins that are differentially expressed in amiR23.6 compared to control plants.

\begin{tabular}{|c|c|c|c|c|c|c|}
\hline Accession & Description & $\begin{array}{c}\text { Coverage } \\
{[\%]}\end{array}$ & $\begin{array}{l}\text { \# Unique } \\
\text { Peptides }\end{array}$ & $\begin{array}{c}\text { Fold } \\
\text { change }\end{array}$ & $\begin{array}{c}\text { Fold } \\
\text { change } \\
\left(\log _{2}\right)\end{array}$ & $\begin{array}{l}\text { Adj. P- } \\
\text { Value }\end{array}$ \\
\hline AT5G65220 & Ribosomal L29 family protein & 27 & 5 & 100 & 6.64 & $1.1 \mathrm{E}-16$ \\
\hline AT1G13900 & Purple acid phosphatases superfamily protein & 5 & 3 & 100 & 6.64 & $1.1 \mathrm{E}-16$ \\
\hline AT5G47190 & Ribosomal protein L19 family protein & 19 & 4 & 100 & 6.64 & $1.1 \mathrm{E}-16$ \\
\hline AT2G21620 & Adenine nucleotide alpha hydrolases-like superfamily protein & 47 & 7 & 100 & 6.64 & 1.1E-16 \\
\hline AT1G74970 & ribosomal protein S9 & 31 & 6 & 100 & 6.64 & $1.1 \mathrm{E}-16$ \\
\hline AT5G03350 & Legume lectin family protein & 20 & 4 & 100 & 6.64 & 1.1E-16 \\
\hline
\end{tabular}


AT5G23010 methylthioalkylmalate synthase 1

AT3G21220 MAP kinase kinase 5

AT3G45140 lipoxygenase 2

AT3G26520 tonoplast intrinsic protein 2

AT5G23900 Ribosomal protein L13e family protein

AT2G20560 DNAJ heat shock family protein

AT1G62480 Vacuolar calcium-binding protein-related

AT1G73030 SNF7 family protein

AT5G62190 DEAD box RNA helicase (PRH75)

AT5G66550 Maf-like protein

AT5G65840 Thioredoxin superfamily protein

\begin{tabular}{c|c|c|c|c|}
$\mathbf{7}$ & $\mathbf{3}$ & $\mathbf{1 0 0}$ & $\mathbf{6 . 6 4}$ & $\mathbf{1 . 1 E}-\mathbf{1 6}$ \\
\hline $\mathbf{4}$ & $\mathbf{2}$ & $\mathbf{1 0 0}$ & $\mathbf{6 . 6 4}$ & $\mathbf{1 . 1 E}-\mathbf{1 6}$ \\
\hline 59 & 39 & 2.20 & 1.14 & $3.0 \mathrm{E}-08$ \\
\hline $\mathbf{1 7}$ & $\mathbf{3}$ & $\mathbf{0 . 4 0}$ & $\mathbf{- 1 . 3 2}$ & $\mathbf{5 . 5 E}-\mathbf{1 1}$ \\
\hline 33 & 2 & 0.31 & -1.67 & $2.4 \mathrm{E}-06$ \\
\hline 35 & 8 & 0.01 & -6.64 & $1.1 \mathrm{E}-16$ \\
\hline $\mathbf{3 8}$ & $\mathbf{2}$ & $\mathbf{0 . 0 1}$ & $\mathbf{- 6 . 6 4}$ & $\mathbf{1 . 1 E}-\mathbf{1 6}$ \\
\hline $\mathbf{1 0}$ & $\mathbf{2}$ & $\mathbf{0 . 0 1}$ & $\mathbf{- 6 . 6 4}$ & $\mathbf{1 . 1 E}-\mathbf{1 6}$ \\
\hline $\mathbf{3}$ & $\mathbf{2}$ & $\mathbf{0 . 0 1}$ & $\mathbf{- 6 . 6 4}$ & $\mathbf{1 . 1 E}-\mathbf{1 6}$ \\
\hline 15 & 2 & 0.01 & -6.64 & $1.1 \mathrm{E}-16$ \\
\hline $\mathbf{8}$ & $\mathbf{2}$ & $\mathbf{0 . 0 1}$ & $\mathbf{- 6 . 6 4}$ & $\mathbf{1 . 1 E}-16$ \\
\hline
\end{tabular}

Suppl. Table 4.3. List of proteins that are differentially expressed in amiR26.5 compared to control plants.

\begin{tabular}{|c|c|c|c|c|c|c|}
\hline Accession & Description & $\begin{array}{l}\text { Coverage } \\
{[\%]}\end{array}$ & $\begin{array}{l}\text { \# Unique } \\
\text { Peptides }\end{array}$ & $\begin{array}{l}\text { Fold } \\
\text { change }\end{array}$ & $\begin{array}{c}\text { Fold } \\
\text { change } \\
\left(\log _{2}\right)\end{array}$ & $\begin{array}{l}\text { Adj. P- } \\
\text { Value }\end{array}$ \\
\hline AT3G10940 & dual specificity protein phosphatase (DsPTP1) family prot. & 7 & 2 & 100 & 6.64 & 6.3E-17 \\
\hline AT5G05270 & Chalcone-flavanone isomerase family protein & 10 & 2 & 100 & 6.64 & 6.3E-17 \\
\hline AT4G18100 & Ribosomal protein L32e & 31 & 3 & 100 & 6.64 & $6.3 \mathrm{E}-17$ \\
\hline AT5G65220 & Ribosomal L29 family protein & 27 & 5 & 100 & 6.64 & $6.3 \mathrm{E}-17$ \\
\hline AT3G09680 & Ribosomal protein S12/S23 family protein & 35 & 3 & 100 & 6.64 & $6.3 \mathrm{E}-17$ \\
\hline AT2G04690 & Pyridoxamine 5'-phosphate oxidase family protein & 15 & 2 & 100 & 6.64 & $6.3 \mathrm{E}-17$ \\
\hline AT5G47190 & Ribosomal protein L19 family protein & 19 & 4 & 100 & 6.64 & $6.3 \mathrm{E}-17$ \\
\hline AT5G19140 & Aluminium induced protein with YGL and LRDR motifs & 46 & 6 & 100 & 6.64 & 6.3E-17 \\
\hline AT4G09650 & ATP synthase delta-subunit gene & 15 & 3 & 100 & 6.64 & $6.3 \mathrm{E}-17$ \\
\hline AT5G14030 & translocon-associated protein beta (TRAPB) family protein & 47 & 4 & 100 & 6.64 & 6.3E-17 \\
\hline AT2G27720 & $60 \mathrm{~S}$ acidic ribosomal protein family & 76 & 3 & 100 & 6.64 & $6.3 \mathrm{E}-17$ \\
\hline AT1G79850 & ribosomal protein $\mathrm{S} 17$ & 31 & 4 & 100 & 6.64 & $6.3 \mathrm{E}-17$ \\
\hline AT2G21620 & Adenine nucleotide alpha hydrolases-like superfamily prot. & 47 & 7 & 100 & 6.64 & $6.3 \mathrm{E}-17$ \\
\hline AT5G13780 & Acyl-CoA N-acyltransferases (NAT) superfamily protein & 11 & 2 & 100 & 6.64 & $6.3 \mathrm{E}-17$ \\
\hline AT1G32470 & Single hybrid motif superfamily protein & 51 & 2 & 100 & 6.64 & $6.3 \mathrm{E}-17$ \\
\hline AT2G21060 & glycine-rich protein $2 \mathrm{~B}$ & 24 & 2 & 100 & 6.64 & 6.3E-17 \\
\hline AT1G17860 & Kunitz family trypsin and protease inhibitor protein & 30 & 4 & 100 & 6.64 & $6.3 \mathrm{E}-17$ \\
\hline AT1G09560 & germin-like protein 5 & 15 & 3 & 100 & 6.64 & $6.3 \mathrm{E}-17$ \\
\hline AT1G20810 & FKBP-like peptidyl-prolyl cis-trans isomerase family protein & 11 & 2 & 100 & 6.64 & $6.3 \mathrm{E}-17$ \\
\hline AT1G21720 & proteasome beta subunit $\mathrm{C} 1$ & 25 & 3 & 100 & 6.64 & $6.3 \mathrm{E}-17$ \\
\hline AT4G17530 & RAB GTPase homolog $1 \mathrm{C}$ & 32 & 3 & 100 & 6.64 & 6.3E-17 \\
\hline AT1G77090 & $\begin{array}{l}\text { Mog1/PsbP/DUF1795-like photosystem II reaction center PsbP family } \\
\text { protein }\end{array}$ & 31 & 5 & 100 & 6.64 & $6.3 \mathrm{E}-17$ \\
\hline AT3G63170 & Chalcone-flavanone isomerase family protein & 21 & 5 & 100 & 6.64 & $6.3 \mathrm{E}-17$ \\
\hline AT1G74970 & ribosomal protein S9 & 31 & 6 & 100 & 6.64 & $6.3 \mathrm{E}-17$ \\
\hline AT4G03280 & photosynthetic electron transfer $\mathrm{C}$ & 14 & 2 & 100 & 6.64 & $6.3 \mathrm{E}-17$ \\
\hline AT4G14800 & $20 S$ proteasome beta subunit $\mathrm{D} 2$ & 20 & 2 & 100 & 6.64 & 6.3E-17 \\
\hline AT3G06050 & peroxiredoxin IIF-PRXIIF & 45 & 7 & 100 & 6.64 & 6.3E-17 \\
\hline AT5G39730 & AIG2-like (avirulence induced gene) family protein & 28 & 4 & 100 & 6.64 & $6.3 \mathrm{E}-17$ \\
\hline AT1G72610 & germin-like protein 1 & 30 & 4 & 100 & 6.64 & $6.3 \mathrm{E}-17$ \\
\hline AT2G22170 & Lipase/lipooxygenase, PLAT/LH2 family protein & 21 & 4 & 100 & 6.64 & $6.3 \mathrm{E}-17$ \\
\hline AT1G65980 & thioredoxin-dependent peroxidase 1-PRXIIB & 62 & 8 & 100 & 6.64 & 6.3E-17 \\
\hline AT2G47910 & chlororespiratory reduction 6 & 26 & 5 & 100 & 6.64 & $6.3 \mathrm{E}-17$ \\
\hline AT3G59870 & unknown protein & 19 & 2 & 100 & 6.64 & $6.3 \mathrm{E}-17$ \\
\hline AT1G20580 & Small nuclear ribonucleoprotein family protein & 13 & 2 & 100 & 6.64 & 6.3E-17 \\
\hline AT5G58060 & SNARE-like superfamily protein & 14 & 3 & 100 & 6.64 & $6.3 \mathrm{E}-17$ \\
\hline AT3G08740 & elongation factor $\mathrm{P}(\mathrm{EF}-\mathrm{P})$ family protein & 38 & 9 & 100 & 6.64 & $6.3 \mathrm{E}-17$ \\
\hline AT3G10520 & haemoglobin 2 & 27 & 4 & 100 & 6.64 & $6.3 \mathrm{E}-17$ \\
\hline AT5G27390 & $\begin{array}{l}\text { Mog1/PsbP/DUF1795-like photosystem II reaction center PsbP family } \\
\text { protein }\end{array}$ & 16 & 4 & 100 & 6.64 & $6.3 \mathrm{E}-17$ \\
\hline AT3G52560 & ubiquitin E2 variant 1D-4 & 32 & 2 & 100 & 6.64 & $6.3 \mathrm{E}-17$ \\
\hline AT2G33120 & synaptobrevin-related protein 1 & 16 & 3 & 100 & 6.64 & $6.3 \mathrm{E}-17$ \\
\hline AT1G11750 & CLP protease proteolytic subunit 6 & 31 & 5 & 100 & 6.64 & $6.3 \mathrm{E}-17$ \\
\hline AT3G07470 & Protein of unknown function, DUF538 & 20 & 3 & 100 & 6.64 & $6.3 \mathrm{E}-17$ \\
\hline AT1G26550 & FKBP-like peptidyl-prolyl cis-trans isomerase family protein & 15 & 2 & 100 & 6.64 & 6.3E-17 \\
\hline AT5G54600 & Translation protein SH3-like family protein & 28 & 6 & 100 & 6.64 & $6.3 \mathrm{E}-17$ \\
\hline ATCG00670 & plastid-encoded CLP P & 21 & 2 & 100 & 6.64 & $6.3 \mathrm{E}-17$ \\
\hline AT5G26667 & $\begin{array}{l}\text { P-loop containing nucleoside triphosphate hydrolases superfamily } \\
\text { protein }\end{array}$ & 23 & 3 & 100 & 6.64 & $6.3 \mathrm{E}-17$ \\
\hline AT3G59400 & enzyme binding;tetrapyrrole binding & 14 & 3 & 100 & 6.64 & $6.3 \mathrm{E}-17$ \\
\hline AT1G13280 & allene oxide cyclase 4 & 17 & 2 & 100 & 6.64 & 6.3E-17 \\
\hline AT3G46000 & actin depolymerizing factor 2 & 24 & 2 & 100 & 6.64 & $6.3 \mathrm{E}-17$ \\
\hline AT3G47810 & Calcineurin-like metallo-phosphoesterase superfamily prot. & 17 & 3 & 100 & 6.64 & $6.3 \mathrm{E}-17$ \\
\hline AT3G53990 & Adenine nucleotide alpha hydrolases-like superfamily prot. & 46 & 5 & 100 & 6.64 & $6.3 \mathrm{E}-17$ \\
\hline AT5G13410 & FKBP-like peptidyl-prolyl cis-trans isomerase family protein & 29 & 5 & 100 & 6.64 & $6.3 \mathrm{E}-17$ \\
\hline AT5G27670 & histone $\mathrm{H} 2 \mathrm{~A} 7$ & 45 & 3 & 100 & 6.64 & $6.3 \mathrm{E}-17$ \\
\hline AT1G24020 & MLP-like protein 423 & 36 & 6 & 100 & 6.64 & $6.3 \mathrm{E}-17$ \\
\hline AT3G62530 & ARM repeat superfamily protein & 43 & 7 & 100 & 6.64 & $6.3 \mathrm{E}-17$ \\
\hline AT2G06050 & oxophytodienoate-reductase 3 & 6 & 2 & 100 & 6.64 & $6.3 \mathrm{E}-17$ \\
\hline AT2G34160 & Alba DNA/RNA-binding protein & 15 & 2 & 100 & 6.64 & $6.3 \mathrm{E}-17$ \\
\hline AT1G03600 & photosystem II family protein & 28 & 4 & 100 & 6.64 & $6.3 \mathrm{E}-17$ \\
\hline AT1G21065 & unknown protein & 9 & 2 & 100 & 6.64 & $6.3 \mathrm{E}-17$ \\
\hline
\end{tabular}




\begin{tabular}{|c|c|c|c|c|c|c|}
\hline AT5G56260 & $\begin{array}{l}\text { Ribonuclease E inhibitor RraA/Dimethylmenaquinone } \\
\text { methyltransferase }\end{array}$ & 13 & 2 & 100 & 6.64 & $6.3 \mathrm{E}-17$ \\
\hline AT2G07707 & Plant mitochondrial ATPase, F0 complex, subunit 8 protein & 13 & 2 & 100 & 6.64 & 6.3E-17 \\
\hline AT3G56070 & rotamase cyclophilin 2 & 25 & 4 & 100 & 6.64 & 6.3E-17 \\
\hline AT4G29350 & profilin 2 & 32 & 3 & 100 & 6.64 & $6.3 \mathrm{E}-17$ \\
\hline AT1G15930 & Ribosomal protein L7Ae/L30e/S12e/Gadd45 family protein & 42 & 3 & 100 & 6.64 & $6.3 \mathrm{E}-17$ \\
\hline AT1G11430 & plastid developmental protein DAG, putative & 17 & 2 & 100 & 6.64 & $6.3 \mathrm{E}-17$ \\
\hline AT3G22230 & Ribosomal L27e protein family & 34 & 2 & 100 & 6.64 & 6.3E-17 \\
\hline AT1G75950 & S phase kinase-associated protein 1 & 29 & 3 & 100 & 6.64 & 6.3E-17 \\
\hline AT5G48760 & Ribosomal protein L13 family protein & 34 & 2 & 100 & 6.64 & 6.3E-17 \\
\hline AT5G28050 & Cytidine/deoxycytidylate deaminase family protein & 21 & 3 & 100 & 6.64 & 6.3E-17 \\
\hline AT1G76450 & Photosystem II reaction center PsbP family protein & 36 & 5 & 100 & 6.64 & 6.3E-17 \\
\hline AT4G11600 & glutathione peroxidase 6 -GPX6 & 22 & 4 & 100 & 6.64 & 6.3E-17 \\
\hline AT2G19740 & Ribosomal protein L31e family protein & 8 & 2 & 100 & 6.64 & 6.3E-17 \\
\hline AT5G08410 & ferredoxin/thioredoxin reductase subunit A 2 & 8 & 2 & 100 & 6.64 & 6.3E-17 \\
\hline AT2G21530 & SMAD/FHA domain-containing protein & 12 & 2 & 100 & 6.64 & 6.3E-17 \\
\hline AT1G16890 & ubiquitin-conjugating enzyme 36 & 10 & 2 & 100 & 6.64 & 6.3E-17 \\
\hline AT1G63970 & isoprenoid $\mathrm{F}$ & 17 & 3 & 100 & 6.64 & 6.3E-17 \\
\hline AT3G60370 & FKBP-like peptidyl-prolyl cis-trans isomerase family protein & 14 & 3 & 100 & 6.64 & 6.3E-17 \\
\hline AT2G16600 & rotamase CYP 3 & 38 & 4 & 100 & 6.64 & 6.3E-17 \\
\hline AT4G34120 & Cystathionine beta-synthase (CBS) family protein & 16 & 3 & 100 & 6.64 & 6.3E-17 \\
\hline AT5G64040 & $\begin{array}{l}\text { photosystem I reaction center subunit PSI-N, chloroplast, putative / } \\
\text { PSI-N, putative (PSAN) }\end{array}$ & 11 & 2 & 100 & 6.64 & $6.3 \mathrm{E}-17$ \\
\hline AT3G25220 & FK506-binding protein $15 \mathrm{kD}-1$ & 16 & 2 & 100 & 6.64 & $6.3 \mathrm{E}-17$ \\
\hline AT5G13120 & cyclophilin $20-2$ & 22 & 4 & 100 & 6.64 & 6.3E-17 \\
\hline AT1G20225 & Thioredoxin superfamily protein & 19 & 3 & 100 & 6.64 & $6.3 \mathrm{E}-17$ \\
\hline AT2G20260 & photosystem I subunit E-2 & 46 & 2 & 100 & 6.64 & $6.3 \mathrm{E}-17$ \\
\hline AT1G29250 & Alba DNA/RNA-binding protein & 37 & 2 & 100 & 6.64 & 6.3E-17 \\
\hline AT1G44790 & ChaC-like family protein & 13 & 2 & 100 & 6.64 & 6.3E-17 \\
\hline AT4G17560 & Ribosomal protein L19 family protein & 16 & 3 & 100 & 6.64 & $6.3 \mathrm{E}-17$ \\
\hline AT1G78630 & Ribosomal protein L13 family protein & 42 & 7 & 89.40 & 6.48 & 6.3E-17 \\
\hline AT5G52650 & RNA binding Plectin/S10 domain-containing protein & 25 & 5 & 52.29 & 5.71 & $6.3 \mathrm{E}-17$ \\
\hline AT5G38430 & Ribulose bisphosphate carboxylase family protein & 69 & 7 & 47.36 & 5.57 & 6.3E-17 \\
\hline AT5G65840 & Thioredoxin superfamily protein & 8 & 2 & 46.34 & 5.53 & 6.3E-17 \\
\hline AT2G43560 & FKBP-like peptidyl-prolyl cis-trans isomerase family protein & 31 & 6 & 43.36 & 5.44 & 6.3E-17 \\
\hline AT5G10860 & Cystathionine beta-synthase (CBS) family protein & 43 & 8 & 36.46 & 5.19 & 6.3E-17 \\
\hline AT4G34870 & rotamase cyclophilin 5 & 42 & 5 & 32.29 & 5.01 & 6.3E-17 \\
\hline AT4G39260 & cold, circadian rhythm, and RNA binding 1 & 51 & 5 & 30.26 & 4.92 & 6.3E-17 \\
\hline AT3G15360 & thioredoxin M-type 4 & 26 & 4 & 29.21 & 4.87 & 6.3E-17 \\
\hline AT4G09320 & Nucleoside diphosphate kinase family protein & 58 & 8 & 29.18 & 4.87 & 6.3E-17 \\
\hline AT2G44650 & chloroplast chaperonin 10 & 37 & 4 & 26.32 & 4.72 & 6.3E-17 \\
\hline AT3G56240 & copper chaperone & 60 & 5 & 26.06 & 4.7 & $6.3 \mathrm{E}-17$ \\
\hline AT5G38410 & Ribulose bisphosphate carboxylase family protein & 69 & 7 & 25.26 & 4.66 & $6.3 \mathrm{E}-17$ \\
\hline AT5G23820 & MD-2-related lipid recognition domain-containing protein & 20 & 5 & 25.15 & 4.65 & $6.3 \mathrm{E}-17$ \\
\hline AT3G16640 & translationally controlled tumor protein & 82 & 12 & 24.25 & 4.6 & 6.3E-17 \\
\hline AT2G04390 & Ribosomal S17 family protein & 30 & 5 & 22.58 & 4.5 & $6.3 \mathrm{E}-17$ \\
\hline AT4G12800 & photosystem I subunit I & 19 & 3 & 21.13 & 4.4 & 6.3E-17 \\
\hline AT4G15000 & Ribosomal L27e protein family & 43 & 4 & 20.77 & 4.38 & 6.3E-17 \\
\hline AT3G11940 & ribosomal protein $5 \mathrm{~A}$ & 56 & 12 & 19.63 & 4.29 & 6.3E-17 \\
\hline AT1G09310 & Protein of unknown function, DUF538 & 52 & 9 & 19.60 & 4.29 & 6.3E-17 \\
\hline AT2G34480 & Ribosomal protein L18ae/LX family protein & 36 & 5 & 18.06 & 4.17 & 6.3E-17 \\
\hline AT2G28720 & Histone superfamily protein & 23 & 3 & 17.79 & 4.15 & 6.3E-17 \\
\hline
\end{tabular}


AT5G61170 Ribosomal protein S19e family protein

AT4G02770 photosystem I subunit D-1

AT1G48350 Ribosomal L18p/L5e family protein

AT1G08110 lactoylglutathione lyase/glyoxalase I family prot.

AT3G60770 Ribosomal protein S13/S15

AT5G59870 histone H2A 6

AT5G16130 Ribosomal protein S7e family protein

AT4G16720 Ribosomal protein L23/L15e family protein

AT3G27830 ribosomal protein L12-A

AT3G44890 ribosomal protein $L 9$

AT5G27850 Ribosomal protein L18e/L15 superfamily protein

AT4G12880 early nodulin-like protein 19

AT5G11770 NADH-ubiquinone oxidoreductase $20 \mathrm{kDa}$ subunit,

AT1G05190 Ribosomal protein L6 family

ATCG00380 chloroplast ribosomal protein S4

AT4G28660 photosystem II reaction center PSB28 protein

AT1G22840 CYTOCHROME C-1

AT1G78370 glutathione S-transferase TAU 20

AT3G55330 PsbP-like protein 1

AT5G06290 2-cysteine peroxiredoxin B-PRXIIB

AT3G02560 Ribosomal protein S7e family protein

AT5G18380 Ribosomal protein S5 domain 2-like superfamily protein

AT2G19760 profilin 1

AT3G48930 Nucleic acid-binding, OB-fold-like protein

AT4G27090 Ribosomal protein L14

AT4G01310 Ribosomal L5P family protein

AT4G11010 nucleoside diphosphate kinase 3

AT3G63190 ribosome recycling factor, chloroplast precursor

AT2G42740 ribosomal protein large subunit 16A

AT5G52840 NADH-ubiquinone oxidoreductase-related

AT1G15820 light harvesting complex photosystem II subunit 6

AT1G13060 20S proteasome beta subunit E1

AT3G04400 Ribosomal protein L14p/L23e family protein

AT5G63310 nucleoside diphosphate kinase 2

AT4G39730 Lipase/lipooxygenase, PLAT/LH2 family protein

AT1G27400 Ribosomal protein L22p/L17e family protein

AT1G69620 ribosomal protein L34

AT3G17020 Adenine nucleotide alpha hydrolases-like superfamily prot.

AT1G14410 ssDNA-binding transcriptional regulator

AT3G24830 Ribosomal protein L13 family protein

AT3G09500 Ribosomal L29 family protein

AT1G54780 thylakoid lumen $18.3 \mathrm{kDa}$ protein

ATCG00900 Ribosomal protein S7p/S5e family protein

AT3G49910 Translation protein SH3-like family protein

AT4G10300 RmIC-like cupins superfamily protein

ATCG00750 ribosomal protein S11

AT2G28490 RmIC-like cupins superfamily protein

AT5G20630 germin 3

AT1G48830 Ribosomal protein S7e family protein

AT1G32990 plastid ribosomal protein I11

AT3G18820 RAB GTPase homolog G3F

AT1G22780 Ribosomal protein S13/S18 family

AT1G35680 Ribosomal protein L21

AT3G05560 Ribosomal L22e protein family

AT1G59860 HSP20-like chaperones superfamily protein

AT1G14320 Ribosomal protein L16p/L10e family protein

AT4G34620 small subunit ribosomal protein 16

AT4G39890 RAB GTPase homolog H1C

AT3G52960 Thioredoxin superfamily protein-PRXIIE

AT3G45140 lipoxygenase 2

AT3G11630 Thioredoxin superfamily protein

AT1G41880 Ribosomal protein L35Ae family protein

AT5G13510 Ribosomal protein L10 family protein

ATCG01120 chloroplast ribosomal protein S15

AT4G33350 Tic22-like family protein

ATCG00780 ribosomal protein L14

AT3G26060 Thioredoxin superfamily protein

AT5G59880 actin depolymerizing factor 3

AT3G18740 Ribosomal protein L7Ae/L30e/S12e/Gadd45 family protein

AT2G39460 ribosomal protein L23AA

AT2G02740 ssDNA-binding transcriptional regulator

AT3G44100 MD-2-related lipid recognition domain-containing protein

AT1G31330 photosystem I subunit F

AT1G70600 Ribosomal protein L18e/L15 superfamily protein

AT4G01480 pyrophosphorylase 5

\begin{tabular}{l|l} 
AT3G26450 & Polyketide cyclase/dehydrase and lipid transport superfamily prot \\
\hline AT3G04920 & Ribosomal protein S24e family protein
\end{tabular}

AT3G61260 Remorin family protein

AT4G23570 phosphatase-related

AT2G20560 DNAJ heat shock family protein

AT1G62480 Vacuolar calcium-binding protein-related

AT5G23900 Ribosomal protein L13e family protein

AT5G56870 beta-galactosidase 4

\begin{tabular}{|l|l|}
\hline 48 & \\
\hline 42 & \\
\hline 25 & \\
\hline 20 & \\
\hline 46 & \\
\hline 52 & \\
\hline 49 & \\
\hline
\end{tabular}


Suppl. Table 4.4. List of proteins that are differentially expressed in amiR23.5/23.6 compared to control plants.

\begin{tabular}{|c|c|c|c|c|c|c|}
\hline Accession & Description & $\begin{array}{l}\text { Coverage } \\
{[\%]}\end{array}$ & $\begin{array}{l}\text { \# Unique } \\
\text { Peptides }\end{array}$ & $\begin{array}{l}\text { Fold } \\
\text { change }\end{array}$ & $\begin{array}{c}\text { Fold } \\
\text { change } \\
\left(\log _{2}\right)\end{array}$ & $\begin{array}{l}\text { Adj. P- } \\
\text { Value }\end{array}$ \\
\hline AT4G18100 & Ribosomal protein L32e & 31 & 3 & 100 & 6.64 & 6.7E-17 \\
\hline AT4G39460 & S-adenosylmethionine carrier 1 & 13 & 2 & 100 & 6.64 & 6.7E-17 \\
\hline AT5G05270 & Chalcone-flavanone isomerase family protein & 10 & 2 & 100 & 6.64 & $6.7 \mathrm{E}-17$ \\
\hline AT3G09680 & Ribosomal protein S12/S23 family protein & 35 & 3 & 100 & 6.64 & 6.7E-17 \\
\hline AT5G47190 & Ribosomal protein L19 family protein & 19 & 4 & 100 & 6.64 & $6.7 \mathrm{E}-17$ \\
\hline AT1G31860 & histidine biosynthesis bifunctional protein (HISIE) & 22 & 3 & 100 & 6.64 & $6.7 \mathrm{E}-17$ \\
\hline AT5G14030 & translocon-associated protein beta (TRAPB) family protein & 47 & 4 & 100 & 6.64 & 6.7E-17 \\
\hline AT2G27720 & $60 \mathrm{~S}$ acidic ribosomal protein family & 76 & 3 & 100 & 6.64 & 6.7E-17 \\
\hline AT1G79850 & ribosomal protein $\mathrm{S} 17$ & 31 & 4 & 100 & 6.64 & $6.7 \mathrm{E}-17$ \\
\hline AT2G21620 & Adenine nucleotide alpha hydrolases-like superfamily prot. & 47 & 7 & 100 & 6.64 & $6.7 \mathrm{E}-17$ \\
\hline AT5G13780 & Acyl-CoA N-acyltransferases (NAT) superfamily protein & 11 & 2 & 100 & 6.64 & 6.7E-17 \\
\hline AT1G32470 & Single hybrid motif superfamily protein & 51 & 2 & 100 & 6.64 & 6.7E-17 \\
\hline AT2G21060 & glycine-rich protein 2B & 24 & 2 & 100 & 6.64 & 6.7E-17 \\
\hline AT1G17860 & Kunitz family trypsin and protease inhibitor protein & 30 & 4 & 100 & 6.64 & 6.7E-17 \\
\hline AT1G09560 & germin-like protein 5 & 15 & 3 & 100 & 6.64 & 6.7E-17 \\
\hline AT1G20810 & FKBP-like peptidyl-prolyl cis-trans isomerase family protein & 11 & 2 & 100 & 6.64 & 6.7E-17 \\
\hline AT1G21720 & proteasome beta subunit $\mathrm{C} 1$ & 25 & 3 & 100 & 6.64 & $6.7 \mathrm{E}-17$ \\
\hline AT4G17530 & RAB GTPase homolog 1C & 32 & 3 & 100 & 6.64 & 6.7E-17 \\
\hline AT1G77090 & $\begin{array}{l}\text { Mog1/PsbP/DUF1795-like photosystem II reaction center PsbP family } \\
\text { protein }\end{array}$ & 31 & 5 & 100 & 6.64 & $6.7 \mathrm{E}-17$ \\
\hline AT3G63170 & Chalcone-flavanone isomerase family protein & 21 & 5 & 100 & 6.64 & 6.7E-17 \\
\hline AT1G74970 & ribosomal protein S9 & 31 & 6 & 100 & 6.64 & 6.7E-17 \\
\hline AT4G03280 & photosynthetic electron transfer C & 14 & 2 & 100 & 6.64 & 6.7E-17 \\
\hline AT1G78630 & Ribosomal protein L13 family protein & 42 & 7 & 100 & 6.64 & 6.7E-17 \\
\hline AT1G02930 & glutathione S-transferase 6 & 64 & 6 & 100 & 6.64 & 6.7E-17 \\
\hline AT4G14800 & 20 S proteasome beta subunit D2 & 20 & 2 & 100 & 6.64 & $6.7 \mathrm{E}-17$ \\
\hline AT1G75270 & dehydroascorbate reductase 2 & 39 & 7 & 100 & 6.64 & 6.7E-17 \\
\hline AT3G06050 & peroxiredoxin IIF-PRXIIF & 45 & 7 & 100 & 6.64 & 6.7E-17 \\
\hline AT5G39730 & AIG2-like (avirulence induced gene) family protein & 28 & 4 & 100 & 6.64 & 6.7E-17 \\
\hline AT1G72610 & germin-like protein 1 & 30 & 4 & 100 & 6.64 & 6.7E-17 \\
\hline AT2G22170 & Lipase/lipooxygenase, PLAT/LH2 family protein & 21 & 4 & 100 & 6.64 & 6.7E-17 \\
\hline AT1G65980 & thioredoxin-dependent peroxidase 1-PRXIIB & 62 & 8 & 100 & 6.64 & 6.7E-17 \\
\hline AT5G65840 & Thioredoxin superfamily protein & 8 & 2 & 100 & 6.64 & 6.7E-17 \\
\hline AT2G47910 & chlororespiratory reduction 6 & 26 & 5 & 100 & 6.64 & $6.7 \mathrm{E}-17$ \\
\hline AT3G59870 & unknown protein & 19 & 2 & 100 & 6.64 & 6.7E-17 \\
\hline AT1G11750 & CLP protease proteolytic subunit 6 & 31 & 5 & 100 & 6.64 & 6.7E-17 \\
\hline AT2G19730 & Ribosomal L28e protein family & 25 & 3 & 100 & 6.64 & $6.7 \mathrm{E}-17$ \\
\hline AT5G58060 & SNARE-like superfamily protein & 14 & 3 & 100 & 6.64 & $6.7 \mathrm{E}-17$ \\
\hline AT3G52560 & ubiquitin E2 variant 1D-4 & 32 & 2 & 100 & 6.64 & 6.7E-17 \\
\hline AT4G28750 & Photosystem I reaction centre subunit IV / PsaE protein & 47 & 2 & 100 & 6.64 & $6.7 \mathrm{E}-17$ \\
\hline AT2G16600 & rotamase CYP 3 & 38 & 4 & 100 & 6.64 & 6.7E-17 \\
\hline AT3G47810 & Calcineurin-like metallo-phosphoesterase superfamily prot. & 17 & 3 & 100 & 6.64 & $6.7 \mathrm{E}-17$ \\
\hline AT1G26550 & FKBP-like peptidyl-prolyl cis-trans isomerase family protein & 15 & 2 & 100 & 6.64 & 6.7E-17 \\
\hline AT3G46000 & actin depolymerizing factor 2 & 24 & 2 & 100 & 6.64 & 6.7E-17 \\
\hline AT5G27390 & $\begin{array}{l}\text { Mog1/PsbP/DUF1795-like photosystem II reaction center PsbP family } \\
\text { protein }\end{array}$ & 16 & 4 & 100 & 6.64 & 6.7E-17 \\
\hline AT1G16890 & ubiquitin-conjugating enzyme 36 & 10 & 2 & 100 & 6.64 & $6.7 \mathrm{E}-17$ \\
\hline AT3G07470 & Protein of unknown function, DUF538 & 20 & 3 & 100 & 6.64 & 6.7E-17 \\
\hline AT3G10520 & haemoglobin 2 & 27 & 4 & 100 & 6.64 & $6.7 \mathrm{E}-17$ \\
\hline AT3G09270 & glutathione S-transferase TAU 8 & 17 & 3 & 100 & 6.64 & $6.7 \mathrm{E}-17$ \\
\hline ATCG00670 & plastid-encoded CLP P & 21 & 2 & 100 & 6.64 & 6.7E-17 \\
\hline AT5G26667 & $\begin{array}{l}\text { P-loop containing nucleoside triphosphate hydrolases superfamily } \\
\text { prot. }\end{array}$ & 23 & 3 & 100 & 6.64 & $6.7 \mathrm{E}-17$ \\
\hline AT1G24020 & MLP-like protein 423 & 36 & 6 & 100 & 6.64 & $6.7 \mathrm{E}-17$ \\
\hline AT3G59400 & enzyme binding;tetrapyrrole binding & 14 & 3 & 100 & 6.64 & $6.7 \mathrm{E}-17$ \\
\hline AT5G27670 & histone $\mathrm{H} 2 \mathrm{~A} 7$ & 45 & 3 & 100 & 6.64 & $6.7 \mathrm{E}-17$ \\
\hline AT1G20580 & Small nuclear ribonucleoprotein family protein & 13 & 2 & 100 & 6.64 & 6.7E-17 \\
\hline AT1G13280 & allene oxide cyclase 4 & 17 & 2 & 100 & 6.64 & $6.7 \mathrm{E}-17$ \\
\hline AT2G35810 & unknown protein & 13 & 2 & 100 & 6.64 & 6.7E-17 \\
\hline AT3G08740 & elongation factor $\mathrm{P}(\mathrm{EF}-\mathrm{P})$ family protein & 38 & 9 & 100 & 6.64 & 6.7E-17 \\
\hline AT3G53990 & Adenine nucleotide alpha hydrolases-like superfamily prot. & 46 & 5 & 100 & 6.64 & 6.7E-17 \\
\hline AT5G13410 & FKBP-like peptidyl-prolyl cis-trans isomerase family protein & 29 & 5 & 100 & 6.64 & 6.7E-17 \\
\hline AT5G54600 & Translation protein SH3-like family protein & 28 & 6 & 100 & 6.64 & $6.7 \mathrm{E}-17$ \\
\hline AT2G34160 & Alba DNA/RNA-binding protein & 15 & 2 & 100 & 6.64 & 6.7E-17 \\
\hline AT1G17880 & basic transcription factor 3 & 28 & 2 & 100 & 6.64 & $6.7 \mathrm{E}-17$ \\
\hline AT1G76450 & Photosystem II reaction center PsbP family protein & 36 & 5 & 100 & 6.64 & $6.7 \mathrm{E}-17$ \\
\hline AT5G28050 & Cytidine/deoxycytidylate deaminase family protein & 21 & 3 & 100 & 6.64 & $6.7 \mathrm{E}-17$ \\
\hline AT1G73230 & Nascent polypeptide-associated complex NAC & 41 & 3 & 100 & 6.64 & 6.7E-17 \\
\hline AT2G20260 & photosystem I subunit E-2 & 46 & 2 & 100 & 6.64 & 6.7E-17 \\
\hline AT2G36145 & unknown protein & 9 & 2 & 100 & 6.64 & $6.7 \mathrm{E}-17$ \\
\hline AT4G23680 & Polyketide cyclase/dehydrase and lipid transport superfamily prot. & 21 & 3 & 100 & 6.64 & 6.7E-17 \\
\hline AT3G22230 & Ribosomal L27e protein family & 34 & 2 & 100 & 6.64 & 6.7E-17 \\
\hline AT3G05590 & ribosomal protein L18 & 52 & 3 & 100 & 6.64 & $6.7 \mathrm{E}-17$ \\
\hline
\end{tabular}


AT1G73260 kunitz trypsin inhibitor 1

\begin{tabular}{l|l|l} 
AT1G73260 & kunitz trypsin inhibitor 1 & 26 \\
\hline AT1G15930 & Ribosomal protein L7Ae/L30e/S12e/Gadd45 family protein & 42
\end{tabular}

\begin{tabular}{|l|r} 
ATIG15930 & Ribosomal protein L7Ae/L30e/S12e/Gadd45 family protein \\
\hline AT2G43945 & unknown protein
\end{tabular}

AT3G56070 rotamase cyclophilin 2

AT2G07707 $\quad$ Plant mitochondrial ATPase, F0 complex, subunit 8 protein

AT1G53670 methionine sulfoxide reductase B 1

AT5G35620 Eukaryotic initiation factor 4E protein

AT5G56260 Ribonuclease E inhibitor RraA/Dimethylmenaquinone

AT1G03600 photosystem II family protein

AT1G11430 plastid developmental protein DAG, putative

\begin{tabular}{l|l}
\hline AT4G11600 & glutathione peroxidase 6-GPX6 \\
\hline
\end{tabular}

AT2G01520 MLP-like protein 328

AT1G20225 Thioredoxin superfamily protein

AT5G13120 cyclophilin 20-2

AT5G08180 Ribosomal protein L7Ae/L30e/S12e/Gadd45 family protein

AT2G33120 synaptobrevin-related protein 1

AT5G08410 ferredoxin/thioredoxin reductase subunit A 2

AT1G29250 Alba DNA/RNA-binding protein

AT2G06050 oxophytodienoate-reductase 3

AT5G23740 ribosomal protein S11-beta

AT1G63970 isoprenoid F

AT3G60370 FKBP-like peptidyl-prolyl cis-trans isomerase family protein

AT4G03520 Thioredoxin superfamily protein

AT5G62350 Plant invertase/pectin methylesterase inhibitor superfamily protein

AT5G48760 Ribosomal protein $L 13$ family protein

AT5G03350 Legume lectin family protein

AT4G34120 Cystathionine beta-synthase (CBS) family pr

AT3G25220 FK506-binding protein $15 \mathrm{kD}$-1

AT3G62530 ARM repeat superfamily protein

AT3G46630 Protein of unknown function (DUF3223)

AT2G19740 Ribosomal protein L31e family protein

AT1G44790 ChaC-like family protein

AT4G17560 Ribosomal protein L19 family protein

AT5G52650 RNA binding Plectin/S10 domain-containing protein

AT5G38430 Ribulose bisphosphate carboxylase family protein

AT4G34870 rotamase cyclophilin 5

AT4G09320 Nucleoside diphosphate kinase family protein

AT5G38410 Ribulose bisphosphate carboxylase family protein

AT1G78370 glutathione S-transferase TAU 20

AT2G04390 Ribosomal S17 family protein

AT1G67090 ribulose bisphosphate carboxylase small chain 1A

AT3G16640 translationally controlled tumor protein

AT2G43560 FKBP-like peptidyl-prolyl cis-trans isomerase family protein

AT3G15360 thioredoxin M-type 4

AT2G27710 60S acidic ribosomal protein family

AT4G39260 cold, circadian rhythm, and RNA binding 1

AT2G34480 Ribosomal protein L18ae/LX family protein

AT1G33140 Ribosomal protein L6 family

AT1G09590 Translation protein SH3-like family protein

AT5G57290 60S acidic ribosomal protein family

AT2G21660 cold, circadian rhythm, and rna binding 2

AT5G40950 ribosomal protein large subunit 27

AT3G56240 copper chaperone

AT3G11940 ribosomal protein 5A

AT1G09310 Protein of unknown function, DUF538

AT5G15200 Ribosomal protein S4

AT5G10860 Cystathionine beta-synthase (CBS) family protein

AT4G15000 Ribosomal L27e protein family

AT2G28190

AT2G28720 Histone superfamily protein

AT3G27830 ribosomal protein L12-A

AT5G23820 MD-2-related lipid recognition domain-containing protein

AT3G62030 rotamase CYP 4

AT4G40030 Histone superfamily protein

AT3G60770 Ribosomal protein S13/S15

AT3G44890 ribosomal protein L9

AT2G35370 glycine decarboxylase complex $\mathrm{H}$

AT1G15820 light harvesting complex photosystem II subunit 6

AT4G21280 photosystem II subunit QA

AT1G06680 photosystem II subunit P-1

ATMG00070 NADH dehydrogenase subunit 9

AT4G27150 seed storage albumin 2

AT4G12880 early nodulin-like protein 19

AT5G27850 Ribosomal protein L18e/L15 superfamily protein

AT5G59870 histone H2A 6

AT5G53490 Tetratricopeptide repeat (TPR)-like superfamily protein

AT1G05190 Ribosomal protein L6 family

AT5G11770 NADH-ubiquinone oxidoreductase $20 \mathrm{kDa}$ subunit

AT3G02560 Ribosomal protein S7e family protein

AT5G61170 Ribosomal protein S19e family protein

AT5G18380 Ribosomal protein S5 domain 2-like superfamily protein

\begin{tabular}{|l|l|}
\hline 26 & \\
\hline 42 & \\
\hline 18 & \\
\hline 25 & \\
\hline 13 & \\
\hline 10 & \\
\hline 15 & \\
\hline 13 & \\
\hline
\end{tabular}

\begin{tabular}{|l|l|}
4 & \\
\hline 3 & \\
2 & \\
\hline 4 & \\
\hline 2 & \\
2 & \\
3 & \\
\hline 2 & \\
\hline 2 & \\
\hline
\end{tabular}

\begin{tabular}{l|l}
100 & 6.64 \\
\hline
\end{tabular}

\begin{tabular}{l|l|l|}
100 & 6.64 & $6.7 \mathrm{E}-17$ \\
\hline 100 & 6.64 & $6.7 \mathrm{E}-17$ \\
\hline
\end{tabular}

\begin{tabular}{l|l|l|}
100 & 6.64 & $6.7 \mathrm{E}-17$ \\
\hline
\end{tabular}

\begin{tabular}{l|l|l|}
100 & 6.64 & $6.7 \mathrm{E}-17$ \\
\hline
\end{tabular}

\begin{tabular}{l|l|l|}
100 & 6.64 & $6.7 \mathrm{E}-17$
\end{tabular}

\begin{tabular}{|l|l|l|}
\hline 100 & 6.64 & $6.7 \mathrm{E}-17$ \\
\hline
\end{tabular}

9
28
7
22
30
19
22
6

4

\begin{tabular}{l|l|l|}
\hline 100 & 6.64 & $6.7 \mathrm{E}-17$ \\
\hline
\end{tabular}

100

100

$6.7 \mathrm{E}-17$

$6.64 \quad 6.7 \mathrm{E}-17$

\begin{tabular}{l|l}
6.64 & $6.7 \mathrm{E}-17$ \\
\hline
\end{tabular}

\begin{tabular}{l|l}
6.64 & $6.7 \mathrm{E}-17$ \\
\hline 6.64 & $6.7 \mathrm{E}-17$
\end{tabular}

\begin{tabular}{l|l}
6.64 & $6.7 \mathrm{E}-17$ \\
\hline 6.64 & $6.7 \mathrm{E}-17$
\end{tabular}

\begin{tabular}{l|l}
6.64 & $6.7 \mathrm{E}-17$ \\
\hline 6.64 & $6.7 \mathrm{E}-17$ \\
\hline
\end{tabular}

$6.64 \quad 6.7 \mathrm{E}-17$

$6.64 \quad 6.7 \mathrm{E}-17$

\begin{tabular}{l|l}
6.64 & $6.7 \mathrm{E}-17$ \\
\hline
\end{tabular}

$6.64 \quad 6.7 \mathrm{E}-17$

$6.64 \quad 6.7 \mathrm{E}-17$

\begin{tabular}{l|l}
6.64 & $6.7 \mathrm{E}-17$ \\
\hline 6.64 & $6.7 \mathrm{E}-17$
\end{tabular}

$6.64 \quad 6.7 \mathrm{E}-17$

6.64

\begin{tabular}{l|l}
6.64 & $6.7 \mathrm{E}-17$ \\
\hline 6.64 & $6.7 \mathrm{E}-17$
\end{tabular}

\begin{tabular}{l|l}
6.64 & $6.7 \mathrm{E}-17$ \\
6.64 & $6.7 \mathrm{E}-17$
\end{tabular}

\begin{tabular}{l|l}
6.64 & $6.7 \mathrm{E}-17$
\end{tabular}

\begin{tabular}{l|l}
6.64 & $6.7 \mathrm{E}-17$ \\
\hline
\end{tabular}

$6.64-6.7 \mathrm{E}-17$

$6.64 \quad 6.7 \mathrm{E}-17$

\begin{tabular}{l|l}
6.64 & $6.7 \mathrm{E}-17$
\end{tabular}

$6.64-6.7 \mathrm{E}-17$

$\begin{array}{ll}6.15 & 6.7 \mathrm{E}-17\end{array}$

\begin{tabular}{ll}
5.68 & $6.7 \mathrm{E}-17$ \\
\hline
\end{tabular}

$\begin{array}{ll}5.33 & 6.7 \mathrm{E}-17 \\ 5.25 & 6.7 \mathrm{E}-17\end{array}$

$5.25 \quad 6.7 \mathrm{E}-17$

\begin{tabular}{|l|l|l|}
\hline 38.08 & 5.25 & $6.7 \mathrm{E}-17$ \\
\hline 35.82 & 5.16 & $6.7 \mathrm{E}-17$ \\
\hline
\end{tabular}

\begin{tabular}{|l|l|l|}
\hline 33.58 & 5.07 & $6.7 \mathrm{E}-17$ \\
\hline
\end{tabular}

\begin{tabular}{l|l|l}
32.62 & 5.03 & $6.7 \mathrm{E}-17$ \\
\hline
\end{tabular}

\begin{tabular}{|c|c|c|}
\hline 32.62 & 5.03 & $6.7 \mathrm{E}-17$ \\
\hline 32.00 & 5 & $6.7 \mathrm{E}-17$ \\
\hline
\end{tabular}

\begin{tabular}{|l|l|l|}
\hline 31.75 & 4.99 & $6.7 \mathrm{E}-17$ \\
\hline
\end{tabular}

\begin{tabular}{l|l|l}
31.25 & 4.97 & $6.7 \mathrm{E}-17$
\end{tabular}

\begin{tabular}{|l|l|l|}
\hline 28.41 & 4.83 & $6.7 \mathrm{E}-17$ \\
\hline
\end{tabular}

\begin{tabular}{|l|l|l|}
\hline 27.40 & 4.78 & $6.7 \mathrm{E}-17$ \\
\hline
\end{tabular}

\begin{tabular}{ll|l}
27.23 & 4.77 & $6.7 \mathrm{E}-17$ \\
\hline
\end{tabular}

\begin{tabular}{l|l|l|}
\hline 22.39 & 4.48 & $6.7 \mathrm{E}-17$
\end{tabular}

\begin{tabular}{|l|l|l|}
\hline 22.39 & 4.48 & $6.7 \mathrm{E}-17$ \\
\hline 22.27 & 4.48 & $6.7 \mathrm{E}-17$ \\
\hline
\end{tabular}

\begin{tabular}{|l|l|l|}
\hline 22.20 & 4.47 & $6.7 \mathrm{E}-17$ \\
\hline
\end{tabular}

\begin{tabular}{l|l|l|}
\hline 20.56 & 4.36 & $6.7 \mathrm{E}-17$ \\
\hline
\end{tabular}

\begin{tabular}{|l|l|l|}
\hline 20.32 & 4.34 & $6.7 \mathrm{E}-17$ \\
\hline
\end{tabular}

\begin{tabular}{l|l|l}
20.21 & 4.34 & $6.7 \mathrm{E}-17$
\end{tabular}

\begin{tabular}{l|l|l|}
\hline 19.98 & 4.32 & $6.7 \mathrm{E}-17$ \\
\hline
\end{tabular}

\begin{tabular}{|l|l|l|}
\hline 19.98 & 4.32 & $6.7 \mathrm{E}-17$ \\
\hline 19.34 & 4.27 & $6.7 \mathrm{E}-17$ \\
\hline
\end{tabular}

\begin{tabular}{l|l|l}
18.77 & 4.23 & $6.7 \mathrm{E}-17$ \\
\hline
\end{tabular}

\begin{tabular}{l|l|l}
\hline 18.28 & 4.19 & $6.7 \mathrm{E}-17$ \\
\hline
\end{tabular}

\begin{tabular}{|l|l|l|}
\hline 18.27 & 4.19 & $6.7 \mathrm{E}-17$ \\
\hline
\end{tabular}

\begin{tabular}{|l|l|l|}
\hline 17.81 & 4.15 & $6.7 \mathrm{E}-17$ \\
\hline
\end{tabular}

\begin{tabular}{l|l|l|}
\hline 17.54 & 4.13 & $6.7 \mathrm{E}-17$ \\
\hline
\end{tabular}

\begin{tabular}{l|l|l|}
\hline 17.03 & 4.09 & $6.7 \mathrm{E}-17$ \\
\hline
\end{tabular}

\begin{tabular}{l|l|l}
\hline 16.95 & 4.08 & $6.7 \mathrm{E}-17$ \\
\hline
\end{tabular}

\begin{tabular}{|l|l|l|}
\hline 16.53 & 4.05 & $6.7 \mathrm{E}-17$ \\
\hline
\end{tabular}

\begin{tabular}{|l|l|l|}
\hline 16.38 & 4.03 & $6.7 \mathrm{E}-17$ \\
\hline
\end{tabular}

\begin{tabular}{|c|c|c|}
\hline 16.38 & 4.03 & $6.7 \mathrm{E}-17$ \\
\hline 16.15 & 4.01 & $6.7 \mathrm{E}-17$ \\
\hline
\end{tabular}

\begin{tabular}{|l|l|l|}
16.02 & 4 & $6.7 \mathrm{E}-17$
\end{tabular}

\begin{tabular}{l|l|l}
15.10 & 3.92 & $6.7 \mathrm{E}-17$ \\
\hline
\end{tabular}

\begin{tabular}{|l|l|l|}
\hline 14.79 & 3.89 & $1.5 \mathrm{E}-15$ \\
\hline
\end{tabular}

\begin{tabular}{|l|l|l|}
\hline 14.35 & 3.84 & $6.7 \mathrm{E}-17$ \\
\hline
\end{tabular}

\begin{tabular}{l|l|l}
14.16 & 3.82 & $6.7 \mathrm{E}-17$ \\
\hline
\end{tabular}

\begin{tabular}{l|l|l}
13.97 & 3.8 & $1.5 \mathrm{E}-15$ \\
\hline
\end{tabular}

\begin{tabular}{l|l|l|}
\hline 13.85 & 3.79 & $1.5 \mathrm{E}-15$ \\
\hline
\end{tabular}

$\begin{array}{lll}13.81 & 3.79 & 2.9 E-15\end{array}$

\begin{tabular}{|l|l|l|}
\hline 13.77 & 3.78 & $6.7 \mathrm{E}-17$ \\
\hline
\end{tabular}

\begin{tabular}{|l|l|l|}
13.77 & 3.78 & $6.7 \mathrm{E}-17$ \\
\hline 13.75 & 3.78 & $6.7 \mathrm{E}-17$ \\
\hline
\end{tabular}

\begin{tabular}{|l|l|l|}
\hline 13.67 & 3.77 & $6.7 \mathrm{E}-17$ \\
\hline
\end{tabular}

\begin{tabular}{l|l|l}
13.39 & 3.74 & $6.7 \mathrm{E}-17$
\end{tabular}

\begin{tabular}{|l|l|l|}
\hline 13.39 & 3.74 & $6.7 \mathrm{E}-17$ \\
\hline 13.15 & 3.72 & $3.9 \mathrm{E}-14$ \\
\hline
\end{tabular}

\begin{tabular}{l|l|l|}
\hline 12.83 & 3.68 & $6.7 \mathrm{E}-17$ \\
\hline
\end{tabular}

\begin{tabular}{ll|l}
12.51 & 3.65 & $6.7 \mathrm{E}-17$
\end{tabular}

\begin{tabular}{|l|l|}
\hline 12.12 & 3.6
\end{tabular}

$6.7 \mathrm{E}-17$ 
AT5G65350 histone 311

AT4G02770 photosystem I subunit D-1

AT4G12800 photosystem I subunit I

AT3G04400 Ribosomal protein L14p/L23e family protein

AT1G08110 lactoylglutathione lyase family protein / glyoxalase I family protein

AT4G27090 Ribosomal protein L14

AT4G16720 Ribosomal protein L23/L15e family protein

AT4G28660 photosystem II reaction center PSB28 protein

AT5G06290 2-cysteine peroxiredoxin B-PRXIIB

AT1G27400 Ribosomal protein L22p/L17e family protein

AT2G28490 RmIC-like cupins superfamily protein

AT5G16130 Ribosomal protein S7e family protein

AT5G10160 Thioesterase superfamily protein

AT3G55330 PsbP-like protein 1

AT3G09500 Ribosomal L29 family protein

AT4G39730 Lipase/lipooxygenase, PLAT/LH2 family protein

AT5G23140 nuclear-encoded CLP protease P7

AT3G48930 Nucleic acid-binding, OB-fold-like protein

AT1G22840 CYTOCHROME C-1

AT5G59880 actin depolymerizing factor 3

AT5G52840 NADH-ubiquinone oxidoreductase-related

AT4G24930 thylakoid lumenal $17.9 \mathrm{kDa}$ protein, chloroplast

AT2G30860 glutathione S-transferase PHI 9

AT3G49910 Translation protein SH3-like family protein

AT1G12410 CLP protease proteolytic subunit 2

AT1G69620 ribosomal protein L34

AT2G42740 ribosomal protein large subunit 16A

AT1G54780 thylakoid lumen $18.3 \mathrm{kDa}$ protein

ATCG00380 chloroplast ribosomal protein S4

AT4G25100 Fe superoxide dismutase 1

AT1G07820 Histone superfamily protein

AT2G19760 profilin 1

AT2G44650 chloroplast chaperonin 10

AT4G10300 RmIC-like cupins superfamily protein

ATCG00750 ribosomal protein S11

AT1G14410 ssDNA-binding transcriptional regulator

ATCG00900 Ribosomal protein S7p/S5e family protein

AT5G63310 nucleoside diphosphate kinase 2

AT3G56650 Mog1/PsbP/DUF1795-like photosystem II reaction center PsbP family protein

AT3G45140 lipoxygenase 2

AT1G13930 Involved in response to salt stress.

AT1G48350 Ribosomal L18p/L5e family protein

AT3G24830 Ribosomal protein L13 family protein

AT3G18820 RAB GTPase homolog G3F

AT1G13060 20S proteasome beta subunit E1

AT2G30620 winged-helix DNA-binding transcription factor family protein

AT3G24500 multiprotein bridging factor $1 \mathrm{C}$

AT4G05180 photosystem II subunit Q-2

AT5G25980 glucoside glucohydrolase 2

AT3G05560 Ribosomal L22e protein family

AT1G48830 Ribosomal protein S7e family protein

AT3G17020 Adenine nucleotide alpha hydrolases-like superfamily prot.

ATCG01120 chloroplast ribosomal protein S15

AT3G54600 Class I glutamine amidotransferase-like superfamily protein

AT1G70600 Ribosomal protein L18e/L15 superfamily protein

AT3G14210 epithiospecifier modifier 1

AT1G14320 Ribosomal protein L16p/L10e family protein

ATCG00780 ribosomal protein L14

AT4G34620 small subunit ribosomal protein 16

AT1G61520 photosystem I light harvesting complex gene 3

AT4G39890 RAB GTPase homolog $\mathrm{H} 1 \mathrm{C}$

AT3G04920 Ribosomal protein S24e family protein

AT3G18740 Ribosomal protein L7Ae/L30e/S12e/Gadd45 family protein

AT1G77940 Ribosomal protein L7Ae/L30e/S12e/Gadd45 family protein

AT1G44575 Chlorophyll A-B binding family protein

AT1G41880 Ribosomal protein L35Ae family protein

AT2G34430 light-harvesting chlorophyll-protein complex II subunit B1

AT3G52960 Thioredoxin superfamily protein-PRXIIE

AT4G02520 glutathione S-transferase PHI 2

AT4G30140 GDSL-like Lipase/Acylhydrolase superfamily protein

AT5G38940 RmIC-like cupins superfamily protein

AT3G63540 Mog1/PsbP/DUF1795-like photosystem II reaction center PsbP family

AT3G63540 protein

AT3G26450 Polyketide cyclase/dehydrase and lipid transport superfamily prot

AT4G31300 N-terminal nucleophile aminohydrolases superfamily protein

AT3G03920 H/ACA ribonucleoprotein complex, subunit Gar1/Naf1 prot.

AT2G30870 glutathione S-transferase PHI 10

AT1G35680 Ribosomal protein L21

AT5G01600 ferretin 1

AT1G03090 methylcrotonyl-CoA carboxylase alpha chain

AT3G06850 2-oxoacid dehydrogenases acyltransferase family protein

AT4G33150 lysine-ketoglutarate reductase/saccharopine dehydrogenase

\begin{tabular}{|c|c|c|c|c|}
\hline 42 & 2 & 11.13 & 3.48 & 6.7E-17 \\
\hline 42 & 8 & 10.87 & 3.44 & $6.7 \mathrm{E}-17$ \\
\hline 19 & 3 & 10.31 & 3.37 & $6.5 \mathrm{E}-12$ \\
\hline 48 & 7 & 10.28 & 3.36 & 6.7E-17 \\
\hline 20 & 5 & 9.84 & 3.3 & 1.3E-12 \\
\hline 56 & 6 & 9.41 & 3.23 & 6.7E-17 \\
\hline 35 & 8 & 9.22 & 3.2 & $6.7 \mathrm{E}-17$ \\
\hline 12 & 2 & 9.05 & 3.18 & 4.7E-09 \\
\hline 58 & 4 & 8.99 & 3.17 & $6.7 \mathrm{E}-17$ \\
\hline 26 & 5 & 8.96 & 3.16 & $6.7 \mathrm{E}-17$ \\
\hline 24 & 8 & 8.68 & 3.12 & $1.6 \mathrm{E}-10$ \\
\hline 49 & 12 & 8.64 & 3.11 & $6.7 \mathrm{E}-17$ \\
\hline 37 & 7 & 8.11 & 3.02 & $1.0 \mathrm{E}-13$ \\
\hline 43 & 8 & 7.92 & 2.99 & $8.6 \mathrm{E}-14$ \\
\hline 31 & 5 & 7.81 & 2.96 & $6.9 \mathrm{E}-12$ \\
\hline 22 & 3 & 7.79 & 2.96 & $5.8 \mathrm{E}-13$ \\
\hline 17 & 3 & 7.78 & 2.96 & $3.1 \mathrm{E}-10$ \\
\hline 49 & 6 & 7.40 & 2.89 & $6.7 \mathrm{E}-17$ \\
\hline 17 & 2 & 7.21 & 2.85 & 4.9E-09 \\
\hline 60 & 5 & 6.88 & 2.78 & $2.0 \mathrm{E}-12$ \\
\hline 24 & 3 & 6.77 & 2.76 & $1.0 \mathrm{E}-08$ \\
\hline 25 & 5 & 6.73 & 2.75 & $2.7 \mathrm{E}-08$ \\
\hline 65 & 13 & 6.53 & 2.71 & $3.5 \mathrm{E}-14$ \\
\hline 41 & 7 & 6.48 & 2.7 & $3.7 \mathrm{E}-11$ \\
\hline 47 & 8 & 6.47 & 2.69 & $6.5 \mathrm{E}-11$ \\
\hline 29 & 2 & 6.44 & 2.69 & $3.5 \mathrm{E}-11$ \\
\hline 64 & 13 & 6.41 & 2.68 & $6.1 \mathrm{E}-14$ \\
\hline 20 & 5 & 6.26 & 2.65 & $9.4 \mathrm{E}-08$ \\
\hline 68 & 10 & 6.04 & 2.59 & 3.7E-13 \\
\hline 26 & 3 & 5.91 & 2.56 & $1.1 \mathrm{E}-10$ \\
\hline 50 & 8 & 5.86 & 2.55 & $1.4 \mathrm{E}-10$ \\
\hline 43 & 4 & 5.64 & 2.5 & 2.0E-05 \\
\hline 37 & 4 & 5.60 & 2.49 & $9.0 \mathrm{E}-06$ \\
\hline 22 & 3 & 5.32 & 2.41 & 5.3E-06 \\
\hline 28 & 5 & 5.21 & 2.38 & $2.5 \mathrm{E}-07$ \\
\hline 21 & 3 & 5.11 & 2.35 & $2.4 \mathrm{E}-05$ \\
\hline 46 & 8 & 5.00 & 2.32 & $7.9 \mathrm{E}-11$ \\
\hline 43 & 9 & 4.88 & 2.29 & 3.8E-09 \\
\hline 39 & 7 & 4.84 & 2.28 & $1.5 \mathrm{E}-07$ \\
\hline 59 & 39 & 4.59 & 2.2 & $7.6 \mathrm{E}-10$ \\
\hline 15 & 2 & 4.59 & 2.2 & $3.7 \mathrm{E}-06$ \\
\hline 25 & 4 & 4.58 & 2.2 & 6.3E-06 \\
\hline 47 & 6 & 4.51 & 2.17 & $3.0 \mathrm{E}-08$ \\
\hline 34 & 5 & 4.50 & 2.17 & $1.1 \mathrm{E}-07$ \\
\hline 34 & 7 & 4.48 & 2.16 & $1.9 \mathrm{E}-06$ \\
\hline 3 & 2 & 4.13 & 2.05 & $1.5 \mathrm{E}-04$ \\
\hline 33 & 4 & 4.08 & 2.03 & 3.7E-05 \\
\hline 46 & 10 & 4.07 & 2.02 & 6.2E-07 \\
\hline 42 & 17 & 3.95 & 1.98 & $3.1 \mathrm{E}-08$ \\
\hline 64 & 2 & 3.94 & 1.98 & $1.2 \mathrm{E}-06$ \\
\hline 57 & 11 & 3.88 & 1.96 & 2.3E-06 \\
\hline 37 & 5 & 3.88 & 1.96 & $1.1 \mathrm{E}-04$ \\
\hline 47 & 5 & 3.86 & 1.95 & 7.1E-05 \\
\hline 8 & 2 & 3.79 & 1.92 & 8.4E-04 \\
\hline 43 & 6 & 3.75 & 1.91 & 4.7E-06 \\
\hline 47 & 13 & 3.59 & 1.84 & $8.8 \mathrm{E}-07$ \\
\hline 32 & 2 & 3.54 & 1.82 & 1.1E-06 \\
\hline 52 & 5 & 3.51 & 1.81 & 7.0E-06 \\
\hline 68 & 6 & 3.35 & 1.75 & $6.0 \mathrm{E}-05$ \\
\hline 27 & 5 & 3.30 & 1.72 & $6.8 \mathrm{E}-04$ \\
\hline 14 & 2 & 3.26 & 1.7 & $3.8 \mathrm{E}-03$ \\
\hline 31 & 3 & 3.25 & 1.7 & $2.8 \mathrm{E}-05$ \\
\hline 48 & 3 & 3.23 & 1.69 & $4.5 \mathrm{E}-06$ \\
\hline 36 & 2 & 3.19 & 1.67 & $3.0 \mathrm{E}-03$ \\
\hline 34 & 7 & 3.05 & 1.61 & 7.4E-05 \\
\hline 16 & 2 & 3.04 & 1.6 & $1.8 \mathrm{E}-03$ \\
\hline 81 & 3 & 2.91 & 1.54 & $1.5 \mathrm{E}-02$ \\
\hline 53 & 12 & 2.89 & 1.53 & 2.1E-05 \\
\hline 81 & 14 & 2.88 & 1.53 & 2.2E-05 \\
\hline 10 & 3 & 2.85 & 1.51 & $2.7 \mathrm{E}-03$ \\
\hline 48 & 3 & 2.82 & 1.5 & $1.1 \mathrm{E}-02$ \\
\hline 50 & 5 & 2.58 & 1.37 & $8.1 \mathrm{E}-03$ \\
\hline 78 & 8 & 2.56 & 1.35 & 9.8E-03 \\
\hline 32 & 7 & 2.52 & 1.33 & 2.9E-03 \\
\hline 14 & 2 & 2.51 & 1.32 & 2.3E-02 \\
\hline 60 & 13 & 2.43 & 1.28 & 4.4E-04 \\
\hline 44 & 10 & 2.40 & 1.26 & $3.1 \mathrm{E}-03$ \\
\hline 33 & 6 & 2.15 & 1.11 & $2.0 \mathrm{E}-02$ \\
\hline 40 & 21 & 0.47 & -1.09 & $4.0 \mathrm{E}-02$ \\
\hline 16 & 6 & 0.41 & -1.29 & $3.3 \mathrm{E}-02$ \\
\hline 12 & 9 & 0.35 & -1.5 & 1.6E-02 \\
\hline
\end{tabular}


AT1G11260 sugar transporter 1

AT5G14450 GDSL-like Lipase/Acylhydrolase superfamily protein

AT4G23570 phosphatase-related

AT5G12110 Glutathione S-transferase, C-terminal-like;Translation elongation

AT1G62480 Vacuolar calcium-binding protein-related

AT2G21370 xylulose kinase-1

AT5G19460 nudix hydrolase homolog 20

AT1G34760 general regulatory factor 11

AT1G70290 trehalose-6-phosphatase synthase S8

AT4G35470 plant intracellular ras group-related LRR 4

\begin{tabular}{c|c|c|c|c|}
6 & 3 & 0.35 & -1.51 & $3.0 \mathrm{E}-02$ \\
\hline 12 & 4 & 0.34 & -1.56 & $1.0 \mathrm{E}-02$ \\
\hline 11 & 3 & 0.01 & -6.64 & $6.7 \mathrm{E}-17$ \\
\hline 23 & 2 & 0.01 & -6.64 & $6.7 \mathrm{E}-17$ \\
\hline 38 & 2 & 0.01 & -6.64 & $6.7 \mathrm{E}-17$ \\
\hline 13 & 4 & 0.01 & -6.64 & $6.7 \mathrm{E}-17$ \\
\hline 7 & 2 & 0.01 & -6.64 & $6.7 \mathrm{E}-17$ \\
\hline 32 & 4 & 0.01 & -6.64 & $6.7 \mathrm{E}-17$ \\
\hline 10 & 2 & 0.01 & -6.64 & $6.7 \mathrm{E}-17$ \\
\hline 4 & 2 & 0.01 & -6.64 & $6.7 \mathrm{E}-17$ \\
\hline
\end{tabular}

Suppl. Table 4.5. List of proteins that are differentially expressed in amiR23.5/23.6/26.5 compared to control plants.

\begin{tabular}{|c|c|c|c|c|c|c|}
\hline Accession & Description & $\begin{array}{l}\text { Coverage } \\
{[\%]}\end{array}$ & $\begin{array}{l}\text { \# Unique } \\
\text { Peptides }\end{array}$ & $\begin{array}{l}\text { Fold } \\
\text { change }\end{array}$ & $\begin{array}{c}\text { Fold } \\
\text { change } \\
\left(\log _{2}\right)\end{array}$ & $\begin{array}{l}\text { Adj. P- } \\
\text { Value }\end{array}$ \\
\hline AT4G18100 & Ribosomal protein L32e & 31 & 3 & 100 & 6.64 & 6.7E-17 \\
\hline AT5G05270 & Chalcone-flavanone isomerase family protein & 10 & 2 & 100 & 6.64 & $6.7 \mathrm{E}-17$ \\
\hline AT2G25950 & Protein of unknown function (DUF1000) & 19 & 2 & 100 & 6.64 & 6.7E-17 \\
\hline AT3G10940 & dual specificity protein phosphatase (DsPTP1) family protein & 7 & 2 & 100 & 6.64 & $6.7 \mathrm{E}-17$ \\
\hline AT3G09680 & Ribosomal protein S12/S23 family protein & 35 & 3 & 100 & 6.64 & 6.7E-17 \\
\hline AT5G47190 & Ribosomal protein L19 family protein & 19 & 4 & 100 & 6.64 & $6.7 \mathrm{E}-17$ \\
\hline AT1G31860 & histidine biosynthesis bifunctional protein (HISIE) & 22 & 3 & 100 & 6.64 & $6.7 \mathrm{E}-17$ \\
\hline AT3G49870 & ADP-ribosylation factor-like A1C & 10 & 2 & 100 & 6.64 & $6.7 \mathrm{E}-17$ \\
\hline AT3G62840 & Small nuclear ribonucleoprotein family protein & 25 & 3 & 100 & 6.64 & $6.7 \mathrm{E}-17$ \\
\hline AT5G14030 & translocon-associated protein beta (TRAPB) family protein & 47 & 4 & 100 & 6.64 & $6.7 \mathrm{E}-17$ \\
\hline AT2G27720 & $60 \mathrm{~S}$ acidic ribosomal protein family & 76 & 3 & 100 & 6.64 & 6.7E-17 \\
\hline AT1G79850 & ribosomal protein $\mathrm{S} 17$ & 31 & 4 & 100 & 6.64 & 6.7E-17 \\
\hline AT2G21620 & Adenine nucleotide alpha hydrolases-like superfamily prot. & 47 & 7 & 100 & 6.64 & $6.7 \mathrm{E}-17$ \\
\hline AT5G13780 & Acyl-CoA N-acyltransferases (NAT) superfamily protein & 11 & 2 & 100 & 6.64 & 6.7E-17 \\
\hline AT1G32470 & Single hybrid motif superfamily protein & 51 & 2 & 100 & 6.64 & 6.7E-17 \\
\hline AT2G21060 & glycine-rich protein $2 \mathrm{~B}$ & 24 & 2 & 100 & 6.64 & $6.7 \mathrm{E}-17$ \\
\hline AT1G17860 & Kunitz family trypsin and protease inhibitor protein & 30 & 4 & 100 & 6.64 & $6.7 \mathrm{E}-17$ \\
\hline AT1G09560 & germin-like protein 5 & 15 & 3 & 100 & 6.64 & $6.7 \mathrm{E}-17$ \\
\hline AT1G20810 & FKBP-like peptidyl-prolyl cis-trans isomerase family protein & 11 & 2 & 100 & 6.64 & $6.7 \mathrm{E}-17$ \\
\hline AT1G21720 & proteasome beta subunit $\mathrm{C} 1$ & 25 & 3 & 100 & 6.64 & $6.7 \mathrm{E}-17$ \\
\hline AT4G17530 & RAB GTPase homolog 1C & 32 & 3 & 100 & 6.64 & $6.7 \mathrm{E}-17$ \\
\hline AT1G77090 & $\begin{array}{l}\text { Mog1/PsbP/DUF1795-like photosystem II reaction center PsbP family } \\
\text { protein }\end{array}$ & 31 & 5 & 100 & 6.64 & $6.7 \mathrm{E}-17$ \\
\hline AT3G63170 & Chalcone-flavanone isomerase family protein & 21 & 5 & 100 & 6.64 & 6.7E-17 \\
\hline AT1G74970 & ribosomal protein S9 & 31 & 6 & 100 & 6.64 & 6.7E-17 \\
\hline AT4G10450 & Ribosomal protein L6 family & 33 & 3 & 100 & 6.64 & $6.7 \mathrm{E}-17$ \\
\hline AT4G03280 & photosynthetic electron transfer $\mathrm{C}$ & 14 & 2 & 100 & 6.64 & $6.7 \mathrm{E}-17$ \\
\hline AT4G14800 & 20 S proteasome beta subunit $\mathrm{D} 2$ & 20 & 2 & 100 & 6.64 & $6.7 \mathrm{E}-17$ \\
\hline AT3G06050 & peroxiredoxin IIF-PRXIIF & 45 & 7 & 100 & 6.64 & 6.7E-17 \\
\hline AT5G39730 & AIG2-like (avirulence induced gene) family protein & 28 & 4 & 100 & 6.64 & $6.7 \mathrm{E}-17$ \\
\hline AT1G72610 & germin-like protein 1 & 30 & 4 & 100 & 6.64 & $6.7 \mathrm{E}-17$ \\
\hline AT2G22170 & Lipase/lipooxygenase, PLAT/LH2 family protein & 21 & 4 & 100 & 6.64 & $6.7 \mathrm{E}-17$ \\
\hline AT1G65980 & thioredoxin-dependent peroxidase 1-PRXIIB & 62 & 8 & 100 & 6.64 & 6.7E-17 \\
\hline AT3G59870 & unknown protein & 19 & 2 & 100 & 6.64 & $6.7 \mathrm{E}-17$ \\
\hline AT2G33120 & synaptobrevin-related protein 1 & 16 & 3 & 100 & 6.64 & $6.7 \mathrm{E}-17$ \\
\hline AT5G13410 & FKBP-like peptidyl-prolyl cis-trans isomerase family protein & 29 & 5 & 100 & 6.64 & $6.7 \mathrm{E}-17$ \\
\hline AT3G10520 & haemoglobin 2 & 27 & 4 & 100 & 6.64 & $6.7 \mathrm{E}-17$ \\
\hline AT1G13280 & allene oxide cyclase 4 & 17 & 2 & 100 & 6.64 & $6.7 \mathrm{E}-17$ \\
\hline AT1G26550 & FKBP-like peptidyl-prolyl cis-trans isomerase family protein & 15 & 2 & 100 & 6.64 & $6.7 \mathrm{E}-17$ \\
\hline AT3G59400 & enzyme binding;tetrapyrrole binding & 14 & 3 & 100 & 6.64 & $6.7 \mathrm{E}-17$ \\
\hline AT3G60370 & FKBP-like peptidyl-prolyl cis-trans isomerase family protein & 14 & 3 & 100 & 6.64 & 6.7E-17 \\
\hline AT3G47810 & Calcineurin-like metallo-phosphoesterase superfamily protein & 17 & 3 & 100 & 6.64 & $6.7 \mathrm{E}-17$ \\
\hline AT1G63970 & isoprenoid $\mathrm{F}$ & 17 & 3 & 100 & 6.64 & $6.7 \mathrm{E}-17$ \\
\hline AT5G28060 & Ribosomal protein S24e family protein & 31 & 2 & 100 & 6.64 & $6.7 \mathrm{E}-17$ \\
\hline AT5G26667 & $\begin{array}{l}\text { P-loop containing nucleoside triphosphate hydrolases superfamily } \\
\text { protein }\end{array}$ & 23 & 3 & 100 & 6.64 & $6.7 \mathrm{E}-17$ \\
\hline AT3G52560 & ubiquitin E2 variant $1 \mathrm{D}-4$ & 32 & 2 & 100 & 6.64 & 6.7E-17 \\
\hline AT3G46000 & actin depolymerizing factor 2 & 24 & 2 & 100 & 6.64 & $6.7 \mathrm{E}-17$ \\
\hline AT2G21530 & SMAD/FHA domain-containing protein & 12 & 2 & 100 & 6.64 & $6.7 \mathrm{E}-17$ \\
\hline AT5G54600 & Translation protein SH3-like family protein & 28 & 6 & 100 & 6.64 & $6.7 \mathrm{E}-17$ \\
\hline AT5G23740 & ribosomal protein S11-beta & 49 & 5 & 100 & 6.64 & 6.7E-17 \\
\hline AT3G08740 & elongation factor $\mathrm{P}(\mathrm{EF}-\mathrm{P})$ family protein & 38 & 9 & 100 & 6.64 & $6.7 \mathrm{E}-17$ \\
\hline AT2G06050 & oxophytodienoate-reductase 3 & 6 & 2 & 100 & 6.64 & $6.7 \mathrm{E}-17$ \\
\hline AT3G07470 & Protein of unknown function, DUF538 & 20 & 3 & 100 & 6.64 & $6.7 \mathrm{E}-17$ \\
\hline AT2G19730 & Ribosomal L28e protein family & 25 & 3 & 100 & 6.64 & $6.7 \mathrm{E}-17$ \\
\hline AT1G11750 & CLP protease proteolytic subunit 6 & 31 & 5 & 100 & 6.64 & $6.7 \mathrm{E}-17$ \\
\hline AT5G27390 & $\begin{array}{l}\text { Mog1/PsbP/DUF1795-like photosystem II reaction center PsbP family } \\
\text { protein }\end{array}$ & 16 & 4 & 100 & 6.64 & $6.7 \mathrm{E}-17$ \\
\hline AT3G53990 & Adenine nucleotide alpha hydrolases-like superfamily prot. & 46 & 5 & 100 & 6.64 & $6.7 \mathrm{E}-17$ \\
\hline AT5G14910 & Heavy metal transport/detoxification superfamily protein & 15 & 2 & 100 & 6.64 & $6.7 \mathrm{E}-17$ \\
\hline AT4G34120 & Cystathionine beta-synthase (CBS) family protein & 16 & 3 & 100 & 6.64 & 6.7E-17 \\
\hline AT2G19740 & Ribosomal protein L31e family protein & 8 & 2 & 100 & 6.64 & 6.7E-17 \\
\hline AT3G46630 & Protein of unknown function (DUF3223) & 9 & 2 & 100 & 6.64 & $6.7 \mathrm{E}-17$ \\
\hline
\end{tabular}


\begin{tabular}{l|l|r} 
AT4G02530 & chloroplast thylakoid lumen protein & 20
\end{tabular}

AT1G60000 RNA-binding (RRM/RBD/RNP motifs) family protein

AT5G48760 Ribosomal protein L13 family protein

AT5G62350 Plant invertase/pectin methylesterase inhibitor superfamily protein

AT4G03520 Thioredoxin superfamily protein

AT1G29250 Alba DNA/RNA-binding protein

AT5G08410 ferredoxin/thioredoxin reductase subunit A 2

AT5G08180 Ribosomal protein L7Ae/L30e/S12e/Gadd45 family protein

AT4G11600 glutathione peroxidase 6-GPX6

AT1G17880 basic transcription factor 3

AT3G09270 glutathione S-transferase TAU 8

AT2G16600 rotamase CYP 3

AT2G35810 unknown protein

AT1G20580 Small nuclear ribonucleoprotein family protein

AT5G27670 histone H2A 7

ATCG00670 plastid-encoded CLP P

AT1G24020 MLP-like protein 423

AT5G37475 Translation initiation factor elF3 subunit

AT5G58060 SNARE-like superfamily protein

AT4G28750 Photosystem I reaction centre subunit IV / PsaE protein

AT5G23010 methylthioalkylmalate synthase 1

AT1G76450 Photosystem II reaction center PsbP family protein

AT5G28050 Cytidine/deoxycytidylate deaminase family protein

AT3G25220 FK506-binding protein $15 \mathrm{kD}-1$

AT3G62530 ARM repeat superfamily protein

AT5G13120 cyclophilin 20-2

AT1G20225 Thioredoxin superfamily protein

AT2G20260 photosystem I subunit E-2

AT2G36145 unknown protein

AT4G23680 Polyketide cyclase/dehydrase and lipid transport superfamily prot.

AT3G05590 ribosomal protein L18

AT1G73260 kunitz trypsin inhibitor 1

AT1G53670 methionine sulfoxide reductase B 1

AT5G35620 Eukaryotic initiation factor 4E protein

AT5G61970 signal recognition particle-related / SRP-related

AT1G23130 Polyketide cyclase/dehydrase and lipid transport superfamily

protein

AT1G03680 thioredoxin M-type 1

AT1G75950 S phase kinase-associated protein 1

AT1G73230 Nascent polypeptide-associated complex NAC

AT3G22230 Ribosomal L27e protein family

AT1G15930 Ribosomal protein L7Ae/L30e/S12e/Gadd45 family protein

AT2G43945 unknown protein

AT3G56070 rotamase cyclophilin 2

AT2G07707 Plant mitochondrial ATPase, F0 complex, subunit 8 protein

AT5G56260 Ribonuclease E inhibitor RraA/Dimethylmenaquinone

AT1G21065 methyltransferase

AT2G34160 Alba DNA/RNA-binding protein

AT1G11430 plastid developmental protein DAG, putative

AT5G47520 RAB GTPase homolog A5A

AT1G44790 ChaC-like family protein

AT4G17560 Ribosomal protein L19 family protein

AT1G78630 Ribosomal protein L13 family protein

AT5G52650 RNA binding Plectin/S10 domain-containing protein

AT5G38430 Ribulose bisphosphate carboxylase family protein

AT2G43560 FKBP-like peptidyl-prolyl cis-trans isomerase family protein

AT4G34870 rotamase cyclophilin 5

AT4G39260 cold, circadian rhythm, and RNA binding 1

AT5G65840 Thioredoxin superfamily protein

AT4G09320 Nucleoside diphosphate kinase family protein

AT5G10860 Cystathionine beta-synthase (CBS) family protein

AT3G16640 translationally controlled tumor protein

AT2G04390 Ribosomal S17 family protein

AT3G11940 ribosomal protein 5A

AT4G15000 Ribosomal L27e protein family

AT2G27710 60S acidic ribosomal protein family

AT3G56240 copper chaperone

AT2G34480 Ribosomal protein L18ae/LX family protein

AT5G15200 Ribosomal protein S4

AT3G15360 thioredoxin M-type 4

AT2G28720 Histone superfamily protein

AT5G57290 60S acidic ribosomal protein family

AT4G02770 photosystem I subunit D-1

AT1G33140 Ribosomal protein L6 family

AT1G09590 Translation protein SH3-like family protein

AT2G21660 cold, circadian rhythm, and rna binding 2

AT5G38410 Ribulose bisphosphate carboxylase family protein

AT4G16720 Ribosomal protein L23/L15e family protein

AT3G60770 Ribosomal protein S13/S15

AT4G40030 Histone superfamily protein

AT5G27850 Ribosomal protein L18e/L15 superfamily protein

AT5G23820 MD-2-related lipid recognition domain-containing protein

\begin{tabular}{|c|c|c|c|c|}
\hline 20 & 3 & 100 & 6.64 & 6.7E-17 \\
\hline 12 & 2 & 100 & 6.64 & $6.7 \mathrm{E}-17$ \\
\hline 34 & 2 & 100 & 6.64 & $6.7 \mathrm{E}-17$ \\
\hline 34 & 6 & 100 & 6.64 & 6.7E-17 \\
\hline 23 & 3 & 100 & 6.64 & 6.7E-17 \\
\hline 37 & 2 & 100 & 6.64 & 6.7E-17 \\
\hline 8 & 2 & 100 & 6.64 & 6.7E-17 \\
\hline 16 & 2 & 100 & 6.64 & $6.7 \mathrm{E}-17$ \\
\hline 22 & 4 & 100 & 6.64 & 6.7E-17 \\
\hline 28 & 2 & 100 & 6.64 & $6.7 \mathrm{E}-17$ \\
\hline 17 & 3 & 100 & 6.64 & $6.7 \mathrm{E}-17$ \\
\hline 38 & 4 & 100 & 6.64 & $6.7 \mathrm{E}-17$ \\
\hline 13 & 2 & 100 & 6.64 & $6.7 \mathrm{E}-17$ \\
\hline 13 & 2 & 100 & 6.64 & 6.7E-17 \\
\hline 45 & 3 & 100 & 6.64 & $6.7 \mathrm{E}-17$ \\
\hline 21 & 2 & 100 & 6.64 & $6.7 \mathrm{E}-17$ \\
\hline 36 & 6 & 100 & 6.64 & 6.7E-17 \\
\hline 20 & 3 & 100 & 6.64 & $6.7 \mathrm{E}-17$ \\
\hline 14 & 3 & 100 & 6.64 & 6.7E-17 \\
\hline 47 & 2 & 100 & 6.64 & 6.7E-17 \\
\hline 7 & 3 & 100 & 6.64 & $6.7 \mathrm{E}-17$ \\
\hline 36 & 5 & 100 & 6.64 & $6.7 \mathrm{E}-17$ \\
\hline 21 & 3 & 100 & 6.64 & $6.7 \mathrm{E}-17$ \\
\hline 16 & 2 & 100 & 6.64 & $6.7 \mathrm{E}-17$ \\
\hline 43 & 7 & 100 & 6.64 & $6.7 \mathrm{E}-17$ \\
\hline 22 & 4 & 100 & 6.64 & $6.7 \mathrm{E}-17$ \\
\hline 19 & 3 & 100 & 6.64 & 6.7E-17 \\
\hline 46 & 2 & 100 & 6.64 & $6.7 \mathrm{E}-17$ \\
\hline 9 & 2 & 100 & 6.64 & 6.7E-17 \\
\hline 21 & 3 & 100 & 6.64 & $6.7 \mathrm{E}-17$ \\
\hline 52 & 3 & 100 & 6.64 & 6.7E-17 \\
\hline 26 & 4 & 100 & 6.64 & $6.7 \mathrm{E}-17$ \\
\hline 10 & 2 & 100 & 6.64 & 6.7E-17 \\
\hline 15 & 3 & 100 & 6.64 & $6.7 \mathrm{E}-17$ \\
\hline 6 & 2 & 100 & 6.64 & $6.7 \mathrm{E}-17$ \\
\hline 21 & 3 & 100 & 6.64 & $6.7 \mathrm{E}-17$ \\
\hline 22 & 3 & 100 & 6.64 & 6.7E-17 \\
\hline 29 & 3 & 100 & 6.64 & $6.7 \mathrm{E}-17$ \\
\hline 41 & 3 & 100 & 6.64 & $6.7 \mathrm{E}-17$ \\
\hline 34 & 2 & 100 & 6.64 & $6.7 \mathrm{E}-17$ \\
\hline 42 & 3 & 100 & 6.64 & 6.7E-17 \\
\hline 18 & 2 & 100 & 6.64 & $6.7 \mathrm{E}-17$ \\
\hline 25 & 4 & 100 & 6.64 & $6.7 \mathrm{E}-17$ \\
\hline 13 & 2 & 100 & 6.64 & $6.7 \mathrm{E}-17$ \\
\hline 13 & 2 & 100 & 6.64 & 6.7E-17 \\
\hline 9 & 2 & 100 & 6.64 & 6.7E-17 \\
\hline 15 & 2 & 100 & 6.64 & $6.7 \mathrm{E}-17$ \\
\hline 17 & 2 & 100 & 6.64 & $6.7 \mathrm{E}-17$ \\
\hline 16 & 2 & 100 & 6.64 & 6.7E-17 \\
\hline 13 & 2 & 100 & 6.64 & $6.7 \mathrm{E}-17$ \\
\hline 16 & 3 & 100 & 6.64 & $6.7 \mathrm{E}-17$ \\
\hline 42 & 7 & 84.97 & 6.41 & $6.7 \mathrm{E}-17$ \\
\hline 25 & 5 & 75.90 & 6.25 & $6.7 \mathrm{E}-17$ \\
\hline 69 & 7 & 48.82 & 5.61 & $6.7 \mathrm{E}-17$ \\
\hline 31 & 6 & 43.96 & 5.46 & 6.7E-17 \\
\hline 42 & 5 & 43.68 & 5.45 & $6.7 \mathrm{E}-17$ \\
\hline 51 & 5 & 41.85 & 5.39 & $6.7 \mathrm{E}-17$ \\
\hline 8 & 2 & 39.06 & 5.29 & $6.7 \mathrm{E}-17$ \\
\hline 58 & 8 & 37.68 & 5.24 & 6.7E-17 \\
\hline 43 & 8 & 34.27 & 5.1 & $6.7 \mathrm{E}-17$ \\
\hline 82 & 12 & 34.02 & 5.09 & $6.7 \mathrm{E}-17$ \\
\hline 30 & 5 & 29.07 & 4.86 & 6.7E-17 \\
\hline 56 & 12 & 25.96 & 4.7 & $6.7 \mathrm{E}-17$ \\
\hline 43 & 4 & 25.83 & 4.69 & $6.7 \mathrm{E}-17$ \\
\hline 68 & 3 & 24.95 & 4.64 & $6.7 \mathrm{E}-17$ \\
\hline 60 & 5 & 24.66 & 4.62 & $6.7 \mathrm{E}-17$ \\
\hline 36 & 5 & 24.44 & 4.61 & $6.7 \mathrm{E}-17$ \\
\hline 53 & 8 & 23.60 & 4.56 & 6.7E-17 \\
\hline 26 & 4 & 21.58 & 4.43 & 6.7E-17 \\
\hline 23 & 3 & 20.99 & 4.39 & $6.7 \mathrm{E}-17$ \\
\hline 22 & 2 & 20.98 & 4.39 & 6.7E-17 \\
\hline 42 & 8 & 20.46 & 4.35 & $6.7 \mathrm{E}-17$ \\
\hline 64 & 12 & 20.19 & 4.34 & 6.7E-17 \\
\hline 45 & 8 & 19.95 & 4.32 & $6.7 \mathrm{E}-17$ \\
\hline 44 & 4 & 18.04 & 4.17 & 6.7E-17 \\
\hline 69 & 7 & 17.21 & 4.1 & 6.7E-17 \\
\hline 35 & 8 & 16.04 & 4 & $6.7 \mathrm{E}-17$ \\
\hline 46 & 9 & 16.02 & 4 & $6.7 \mathrm{E}-17$ \\
\hline 36 & 2 & 15.79 & 3.98 & $6.7 \mathrm{E}-17$ \\
\hline 59 & 5 & 14.83 & 3.89 & 6.7E-17 \\
\hline 20 & 5 & 14.76 & 3.88 & 6.7E-17 \\
\hline
\end{tabular}


AT5G61170 Ribosomal protein S19e family protein

AT5G10160 Thioesterase superfamily protein

AT3G62030 rotamase CYP 4

AT2G28190 copper/zinc superoxide dismutase 2

AT5G40950 ribosomal protein large subunit 27

AT1G09310 Protein of unknown function, DUF538

AT1G78370 glutathione S-transferase TAU 20

ATMG00070 NADH dehydrogenase subunit 9

AT4G12800 photosystem I subunit I

AT1G05190 Ribosomal protein L6 family

AT5G16130 Ribosomal protein S7e family protein

AT1G06680 photosystem II subunit P-1

AT2G35370 glycine decarboxylase complex $\mathrm{H}$

AT5G11770 NADH-ubiquinone oxidoreductase $20 \mathrm{kDa}$ subunit,

AT4G12880 early nodulin-like protein 19

AT1G67090 ribulose bisphosphate carboxylase small chain $1 \mathrm{~A}$

AT5G53490 Tetratricopeptide repeat (TPR)-like superfamily protein

AT3G44890 ribosomal protein L9

AT2G44120 Ribosomal protein L30/L7 family protein

AT5G18380 Ribosomal protein S5 domain 2-like superfamily protein

AT5G65350 histone 311

AT2G44650 chloroplast chaperonin 10

AT1G22840 CYTOCHROME C-1

AT3G27830 ribosomal protein L12-A

AT1G27400 Ribosomal protein L22p/L17e family protein

AT5G59870 histone H2A 6

AT1G08110 lactoylglutathione lyase family protein / glyoxalase I family protein

AT3G02560 Ribosomal protein S7e family protein

AT4G27090 Ribosomal protein L14

AT3G04400 Ribosomal protein L14p/L23e family protein

AT2G42740 ribosomal protein large subunit 16A

AT3G09500 Ribosomal L29 family protein

AT3G48930 Nucleic acid-binding, OB-fold-like protein

AT4G21280 photosystem II subunit QA

AT4G28660 photosystem II reaction center PSB28 protein

ATCG00380 chloroplast ribosomal protein S4

AT5G52840 NADH-ubiquinone oxidoreductase-related

AT4G39730 Lipase/lipooxygenase, PLAT/LH2 family protein

AT1G54780 thylakoid lumen $18.3 \mathrm{kDa}$ protein

AT3G24830 Ribosomal protein L13 family protein

AT1G69620 ribosomal protein L34

AT2G19760 profilin 1

AT3G49910 Translation protein SH3-like family protein

AT1G07820 Histone superfamily protein

AT5G06290 2-cysteine peroxiredoxin B-PRXIIB

AT3G55330 PsbP-like protein 1

AT1G15820 light harvesting complex photosystem II subunit 6

AT1G17170 glutathione S-transferase TAU 24

AT2G30620 winged-helix DNA-binding transcription factor family protein

ATCG00750 ribosomal protein S11

AT1G48830 Ribosomal protein S7e family protein

AT1G14410 ssDNA-binding transcriptional regulator

AT1G13060 20S proteasome beta subunit E1

AT3G63190 ribosome recycling factor, chloroplast precursor

ATCG00900 Ribosomal protein S7p/S5e family protein

AT5G63310 nucleoside diphosphate kinase 2

AT1G22780 Ribosomal protein S13/S18 family

AT1G14320 Ribosomal protein L16p/L10e family protein

AT3G14210 epithiospecifier modifier 1

AT1G12410 CLP protease proteolytic subunit 2

AT3G17020 Adenine nucleotide alpha hydrolases-like superfamily prot.

AT4G39890 RAB GTPase homolog H1C

AT4G34620 small subunit ribosomal protein 16

AT1G70600 Ribosomal protein L18e/L15 superfamily protein

AT3G18820 RAB GTPase homolog G3F

AT1G02780 Ribosomal protein L19e family protein

AT3G54600 Class I glutamine amidotransferase-like superfamily protein

AT5G25980 glucoside glucohydrolase 2

AT3G04920 Ribosomal protein S24e family protein

ATCG01120 chloroplast ribosomal protein S15

AT3G53460 chloroplast RNA-binding protein 29

AT1G41880 Ribosomal protein L35Ae family protein

AT1G77940 Ribosomal protein L7Ae/L30e/S12e/Gadd45 family protein

ATCG00780 ribosomal protein L14

AT1G71500 Rieske (2Fe-2S) domain-containing protein

ATCG00650 ribosomal protein S18

AT3G13120 Ribosomal protein S10p/S20e family protein

AT4G10300 RmIC-like cupins superfamily protein

AT3G52960 Thioredoxin superfamily protein-PRXIIE

AT3G18740 Ribosomal protein L7Ae/L30e/S12e/Gadd45 famil

AT4G17170 RAB GTPase homolog B1C

AT4G33350 Tic22-like family protein

AT4G14710 RmIC-like cupins superfamily protein

\begin{tabular}{|c|c|c|c|c|}
\hline 48 & 5 & 14.38 & 3.85 & 6.7E-17 \\
\hline 37 & 7 & 14.21 & 3.83 & 6.7E-17 \\
\hline 39 & 9 & 14.19 & 3.83 & $6.7 \mathrm{E}-17$ \\
\hline 24 & 3 & 14.17 & 3.82 & $6.7 \mathrm{E}-17$ \\
\hline 23 & 4 & 14.07 & 3.81 & $6.7 \mathrm{E}-17$ \\
\hline 52 & 9 & 14.07 & 3.81 & $6.7 \mathrm{E}-17$ \\
\hline 38 & 10 & 13.91 & 3.8 & $6.7 \mathrm{E}-17$ \\
\hline 17 & 3 & 13.82 & 3.79 & $6.7 \mathrm{E}-17$ \\
\hline 19 & 3 & 13.33 & 3.74 & 6.7E-17 \\
\hline 62 & 20 & 13.32 & 3.74 & $6.7 \mathrm{E}-17$ \\
\hline 49 & 12 & 13.26 & 3.73 & 6.7E-17 \\
\hline 59 & 10 & 12.45 & 3.64 & 2.9E-15 \\
\hline 30 & 2 & 11.89 & 3.57 & 1.0E-14 \\
\hline 9 & 2 & 11.69 & 3.55 & 1.6E-14 \\
\hline 11 & 2 & 11.65 & 3.54 & 1.7E-14 \\
\hline 64 & 15 & 11.47 & 3.52 & 2.6E-14 \\
\hline 39 & 9 & 11.47 & 3.52 & 2.6E-14 \\
\hline 47 & 10 & 11.46 & 3.52 & 2.6E-14 \\
\hline 66 & 4 & 10.80 & 3.43 & $1.2 E-13$ \\
\hline 50 & 2 & 10.77 & 3.43 & 1.3E-13 \\
\hline 42 & 2 & 10.66 & 3.41 & 1.7E-13 \\
\hline 37 & 4 & 10.36 & 3.37 & 3.3E-13 \\
\hline 17 & 2 & 10.35 & 3.37 & $3.4 \mathrm{E}-13$ \\
\hline 20 & 4 & 10.04 & 3.33 & 7.2E-13 \\
\hline 26 & 5 & 9.99 & 3.32 & $8.1 \mathrm{E}-13$ \\
\hline 52 & 5 & 9.80 & 3.29 & 1.3E-12 \\
\hline 20 & 5 & 9.79 & 3.29 & 1.3E-12 \\
\hline 54 & 8 & 9.35 & 3.22 & $3.9 E-12$ \\
\hline 56 & 6 & 9.30 & 3.22 & $4.4 \mathrm{E}-12$ \\
\hline 48 & 7 & 8.81 & 3.14 & $1.5 \mathrm{E}-11$ \\
\hline 64 & 13 & 8.76 & 3.13 & 1.7E-11 \\
\hline 31 & 5 & 8.14 & 3.02 & $9.1 \mathrm{E}-11$ \\
\hline 49 & 6 & 7.54 & 2.91 & 4.7E-10 \\
\hline 52 & 15 & 7.52 & 2.91 & $5.0 \mathrm{E}-10$ \\
\hline 12 & 2 & 7.40 & 2.89 & 1.1E-08 \\
\hline 68 & 10 & 7.28 & 2.86 & $9.8 \mathrm{E}-10$ \\
\hline 24 & 3 & 6.91 & 2.79 & 2.9E-09 \\
\hline 22 & 3 & 6.64 & 2.73 & $6.4 \mathrm{E}-09$ \\
\hline 20 & 5 & 6.17 & 2.62 & 2.8E-08 \\
\hline 47 & 6 & 6.13 & 2.62 & $3.1 \mathrm{E}-08$ \\
\hline 29 & 2 & 6.06 & 2.6 & $3.9 E-08$ \\
\hline 43 & 4 & 5.83 & 2.54 & 9.3E-07 \\
\hline 41 & 7 & 5.71 & 2.51 & 1.2E-07 \\
\hline 50 & 8 & 5.57 & 2.48 & 1.9E-07 \\
\hline 58 & 4 & 5.23 & 2.39 & 5.7E-07 \\
\hline 43 & 8 & 5.23 & 2.39 & 5.8E-07 \\
\hline 19 & 5 & 5.04 & 2.33 & $1.1 \mathrm{E}-06$ \\
\hline 11 & 2 & 4.90 & 2.29 & $1.8 \mathrm{E}-06$ \\
\hline 3 & 2 & 4.89 & 2.29 & $1.8 \mathrm{E}-06$ \\
\hline 28 & 5 & 4.89 & 2.29 & $1.8 \mathrm{E}-06$ \\
\hline 57 & 11 & 4.78 & 2.26 & $2.6 \mathrm{E}-06$ \\
\hline 21 & 3 & 4.69 & 2.23 & $4.8 \mathrm{E}-06$ \\
\hline 34 & 7 & 4.68 & 2.23 & 3.7E-06 \\
\hline 26 & 6 & 4.62 & 2.21 & 4.7E-06 \\
\hline 46 & 8 & 4.62 & 2.21 & 4.7E-06 \\
\hline 43 & 9 & 4.62 & 2.21 & 4.7E-06 \\
\hline 39 & 6 & 4.53 & 2.18 & $6.4 \mathrm{E}-06$ \\
\hline 32 & 2 & 4.42 & 2.14 & 9.3E-06 \\
\hline 47 & 13 & 4.32 & 2.11 & 1.3E-05 \\
\hline 47 & 8 & 4.31 & 2.11 & 1.4E-05 \\
\hline 37 & 5 & 4.29 & 2.1 & $1.5 \mathrm{E}-05$ \\
\hline 14 & 2 & 4.26 & 2.09 & 2.4E-05 \\
\hline 68 & 6 & 4.13 & 2.05 & 2.6E-05 \\
\hline 43 & 6 & 3.90 & 1.97 & $6.1 \mathrm{E}-05$ \\
\hline 34 & 5 & 3.79 & 1.92 & 9.3E-05 \\
\hline 29 & 5 & 3.72 & 1.89 & 1.2E-04 \\
\hline 8 & 2 & 3.50 & 1.81 & 5.7E-04 \\
\hline 42 & 17 & 3.41 & 1.77 & $3.9 \mathrm{E}-04$ \\
\hline 31 & 3 & 3.32 & 1.73 & 5.5E-04 \\
\hline 47 & 5 & 3.32 & 1.73 & 5.7E-04 \\
\hline 32 & 7 & 3.28 & 1.72 & $6.4 \mathrm{E}-04$ \\
\hline 16 & 2 & 3.28 & 1.71 & $6.4 \mathrm{E}-04$ \\
\hline 36 & 2 & 3.25 & 1.7 & 7.3E-04 \\
\hline 52 & 5 & 3.03 & 1.6 & 1.7E-03 \\
\hline 42 & 9 & 3.00 & 1.58 & $1.9 \mathrm{E}-03$ \\
\hline 33 & 3 & 2.77 & 1.47 & $4.8 \mathrm{E}-03$ \\
\hline 15 & 3 & 2.76 & 1.46 & $5.0 \mathrm{E}-03$ \\
\hline 22 & 3 & 2.75 & 1.46 & 8.1E-03 \\
\hline 53 & 12 & 2.72 & 1.44 & $5.8 \mathrm{E}-03$ \\
\hline 48 & 3 & 2.67 & 1.42 & 7.1E-03 \\
\hline 56 & 3 & 2.60 & 1.38 & 9.3E-03 \\
\hline 20 & 5 & 2.55 & 1.35 & $1.1 \mathrm{E}-02$ \\
\hline 30 & 6 & 2.31 & 1.21 & 3.0E-02 \\
\hline
\end{tabular}


AT4G01480 pyrophosphorylase 5

AT3G07110 Ribosomal protein $L 13$ family protein

AT1G74050 Ribosomal protein L6 family protein

AT1G30730 FAD-binding Berberine family protein

AT1G76180 Dehydrin family protein

AT5G64290 dicarboxylate transport 2.1

AT5G09660 peroxisomal NAD-malate dehydrogenase 2

AT1G01900 subtilase family protein

AT5G45690 Protein of unknown function (DUF1264)

AT5G16620 hydroxyproline-rich glycoprotein family protein

AT4G23570 phosphatase-related

AT1G62480 Vacuolar calcium-binding protein-related

AT3G08010 RNA binding

AT4G35470 plant intracellular ras group-related LRR 4

\begin{tabular}{|c|c|}
\hline 13 & \\
\hline 41 & \\
\hline 58 & \\
\hline 30 & \\
\hline 25 & \\
\hline 7 & \\
\hline 66 & \\
\hline 6 & \\
\hline 16 & \\
\hline 8 & \\
\hline 11 & \\
\hline 38 & \\
\hline 14 & \\
\hline 4 & \\
\hline
\end{tabular}

\begin{tabular}{|c|c|}
\hline 2 & 2 \\
\hline 5 & 2 \\
\hline 7 & 2 \\
\hline 10 & 0 \\
\hline $\mathbf{2}$ & 0 \\
\hline $\mathbf{3}$ & 0 \\
$\mathbf{1 3}$ & 0 \\
\hline $\mathbf{4}$ & 0 \\
\hline 2 & 0 \\
\hline 3 & 0 \\
\hline 3 & 0 \\
\hline 2 & 0 \\
\hline 3 & 0 \\
\hline 2 & 0 \\
\hline
\end{tabular}

Suppl. Table 4.6. List of proteins that are differentially expressed in amiR23.5 compared to control plants after heat treatment.

\begin{tabular}{|c|c|c|c|c|c|c|}
\hline Accession & Description & $\begin{array}{l}\text { Coverage } \\
{[\%]}\end{array}$ & $\begin{array}{l}\text { \# Unique } \\
\text { Peptides }\end{array}$ & $\begin{array}{l}\text { Fold } \\
\text { change }\end{array}$ & $\begin{array}{c}\text { Fold } \\
\text { change } \\
\left(\log _{2}\right)\end{array}$ & $\begin{array}{l}\text { Adj. P- } \\
\text { Value }\end{array}$ \\
\hline AT4G18100 & Ribosomal protein L32e & 31 & 3 & 100 & 6.64 & $8.2 \mathrm{E}-17$ \\
\hline AT1G54050 & HSP20-like chaperones superfamily protein & 54 & 10 & 100 & 6.64 & 8.2E-17 \\
\hline AT3G56290 & unknown protein & 17 & 2 & 100 & 6.64 & $8.2 \mathrm{E}-17$ \\
\hline AT5G35680 & Nucleic acid-binding, OB-fold-like protein & 25 & 3 & 100 & 6.64 & 8.2E-17 \\
\hline AT2G04690 & Pyridoxamine 5'-phosphate oxidase family protein & 15 & 2 & 100 & 6.64 & 8.2E-17 \\
\hline ATCG00820 & ribosomal protein $\mathrm{S} 19$ & 40 & 4 & 100 & 6.64 & $8.2 \mathrm{E}-17$ \\
\hline AT3G02080 & Ribosomal protein S19e family protein & 45 & 4 & 100 & 6.64 & 8.2E-17 \\
\hline ATCG00810 & ribosomal protein L22 & 40 & 5 & 100 & 6.64 & $8.2 \mathrm{E}-17$ \\
\hline AT3G57180 & $\begin{array}{l}\text { P-loop containing nucleoside triphosphate hydrolases superfamily } \\
\text { protein }\end{array}$ & 3 & 2 & 100 & 6.64 & 8.2E-17 \\
\hline AT2G27720 & $60 S$ acidic ribosomal protein family & 76 & 3 & 100 & 6.64 & $8.2 \mathrm{E}-17$ \\
\hline AT4G03280 & photosynthetic electron transfer $\mathrm{C}$ & 14 & 2 & 100 & 6.64 & 8.2E-17 \\
\hline AT4G12800 & photosystem I subunit I & 19 & 3 & 100 & 6.64 & $8.2 \mathrm{E}-17$ \\
\hline AT5G53490 & Tetratricopeptide repeat (TPR)-like superfamily protein & 39 & 9 & 100 & 6.64 & $8.2 \mathrm{E}-17$ \\
\hline AT2G21530 & SMAD/FHA domain-containing protein & 12 & 2 & 100 & 6.64 & $8.2 \mathrm{E}-17$ \\
\hline AT1G63970 & isoprenoid $\mathrm{F}$ & 17 & 3 & 100 & 6.64 & $8.2 \mathrm{E}-17$ \\
\hline AT3G53990 & Adenine nucleotide alpha hydrolases-like superfamily protein & 46 & 5 & 100 & 6.64 & $8.2 \mathrm{E}-17$ \\
\hline AT4G28750 & Photosystem I reaction centre subunit IV / PsaE protein & 47 & 2 & 100 & 6.64 & $8.2 \mathrm{E}-17$ \\
\hline AT3G46000 & actin depolymerizing factor 2 & 24 & 2 & 100 & 6.64 & $8.2 \mathrm{E}-17$ \\
\hline AT5G28060 & Ribosomal protein S24e family protein & 31 & 2 & 100 & 6.64 & $8.2 \mathrm{E}-17$ \\
\hline AT3G10520 & haemoglobin 2 & 27 & 4 & 100 & 6.64 & $8.2 \mathrm{E}-17$ \\
\hline AT2G44920 & Tetratricopeptide repeat (TPR)-like superfamily protein & 36 & 5 & 100 & 6.64 & $8.2 \mathrm{E}-17$ \\
\hline AT3G07470 & Protein of unknown function, DUF538 & 20 & 3 & 100 & 6.64 & $8.2 \mathrm{E}-17$ \\
\hline AT3G52560 & ubiquitin E2 variant 1D-4 & 32 & 2 & 100 & 6.64 & $8.2 \mathrm{E}-17$ \\
\hline AT1G26550 & FKBP-like peptidyl-prolyl cis-trans isomerase family protein & 15 & 2 & 100 & 6.64 & $8.2 \mathrm{E}-17$ \\
\hline AT1G75350 & Ribosomal protein L31 & 32 & 4 & 100 & 6.64 & $8.2 \mathrm{E}-17$ \\
\hline ATCG00670 & plastid-encoded CLP P & 21 & 2 & 100 & 6.64 & $8.2 \mathrm{E}-17$ \\
\hline AT1G11430 & plastid developmental protein DAG, putative & 17 & 2 & 100 & 6.64 & $8.2 \mathrm{E}-17$ \\
\hline AT4G38600 & HEAT repeat ;HECT-domain (ubiquitin-transferase) & 1 & 2 & 100 & 6.64 & $8.2 \mathrm{E}-17$ \\
\hline AT5G61970 & signal recognition particle-related / SRP-related & 6 & 2 & 100 & 6.64 & $8.2 \mathrm{E}-17$ \\
\hline AT1G76450 & Photosystem II reaction center PsbP family protein & 36 & 5 & 100 & 6.64 & $8.2 \mathrm{E}-17$ \\
\hline AT5G51110 & Transcriptional coactivator/pterin dehydratase & 12 & 2 & 100 & 6.64 & $8.2 \mathrm{E}-17$ \\
\hline AT1G29250 & Alba DNA/RNA-binding protein & 37 & 2 & 100 & 6.64 & $8.2 \mathrm{E}-17$ \\
\hline AT1G17880 & basic transcription factor 3 & 28 & 2 & 100 & 6.64 & $8.2 \mathrm{E}-17$ \\
\hline AT1G20580 & Small nuclear ribonucleoprotein family protein & 13 & 2 & 100 & 6.64 & $8.2 \mathrm{E}-17$ \\
\hline AT1G08880 & Histone superfamily protein & 45 & 2 & 100 & 6.64 & $8.2 \mathrm{E}-17$ \\
\hline AT3G22230 & Ribosomal L27e protein family & 34 & 2 & 100 & 6.64 & $8.2 \mathrm{E}-17$ \\
\hline AT3G10090 & Nucleic acid-binding, OB-fold-like protein & 23 & 2 & 100 & 6.64 & $8.2 \mathrm{E}-17$ \\
\hline AT3G25220 & FK506-binding protein $15 \mathrm{kD}-1$ & 16 & 2 & 100 & 6.64 & $8.2 \mathrm{E}-17$ \\
\hline AT2G36145 & unknown protein & 9 & 2 & 100 & 6.64 & $8.2 \mathrm{E}-17$ \\
\hline AT4G23680 & Polyketide cyclase/dehydrase and lipid transport superfamily prot. & 21 & 3 & 100 & 6.64 & $8.2 \mathrm{E}-17$ \\
\hline AT3G05590 & ribosomal protein L18 & 52 & 3 & 100 & 6.64 & $8.2 \mathrm{E}-17$ \\
\hline AT2G33530 & serine carboxypeptidase-like 46 & 4 & 2 & 100 & 6.64 & $8.2 \mathrm{E}-17$ \\
\hline AT4G28660 & photosystem II reaction center PSB28 protein & 12 & 2 & 100 & 6.64 & $8.2 \mathrm{E}-17$ \\
\hline AT2G34160 & Alba DNA/RNA-binding protein & 15 & 2 & 100 & 6.64 & $8.2 \mathrm{E}-17$ \\
\hline AT1G03600 & photosystem II family protein & 28 & 4 & 100 & 6.64 & $8.2 \mathrm{E}-17$ \\
\hline AT1G21065 & unknown protein & 9 & 2 & 100 & 6.64 & $8.2 \mathrm{E}-17$ \\
\hline AT5G14910 & Heavy metal transport/detoxification superfamily protein & 15 & 2 & 100 & 6.64 & $8.2 \mathrm{E}-17$ \\
\hline AT4G29350 & profilin 2 & 32 & 3 & 100 & 6.64 & $8.2 \mathrm{E}-17$ \\
\hline AT1G15930 & Ribosomal protein L7Ae/L30e/S12e/Gadd45 family protein & 42 & 3 & 100 & 6.64 & $8.2 \mathrm{E}-17$ \\
\hline AT1G23130 & Polyketide cyclase/dehydrase and lipid transport superfamily prot. & 21 & 3 & 100 & 6.64 & $8.2 \mathrm{E}-17$ \\
\hline AT2G38540 & lipid transfer protein 1 & 22 & 2 & 100 & 6.64 & $8.2 \mathrm{E}-17$ \\
\hline AT1G31860 & histidine biosynthesis bifunctional protein (HISIE) & 22 & 3 & 74.10 & 6.21 & $8.2 \mathrm{E}-17$ \\
\hline AT4G09320 & Nucleoside diphosphate kinase family protein & 58 & 8 & 73.67 & 6.2 & $8.2 \mathrm{E}-17$ \\
\hline AT5G65840 & Thioredoxin superfamily protein & 8 & 2 & 58.76 & 5.88 & $8.2 \mathrm{E}-17$ \\
\hline AT3G44890 & ribosomal protein L9 & 47 & 10 & 38.08 & 5.25 & $8.2 \mathrm{E}-17$ \\
\hline AT1G07820 & Histone superfamily protein & 50 & 8 & 33.02 & 5.05 & $8.2 \mathrm{E}-17$ \\
\hline AT1G67090 & ribulose bisphosphate carboxylase small chain $1 \mathrm{~A}$ & 64 & 15 & 27.98 & 4.81 & $8.2 \mathrm{E}-17$ \\
\hline AT1G79850 & ribosomal protein S17 & 31 & 4 & 26.25 & 4.71 & $8.2 \mathrm{E}-17$ \\
\hline
\end{tabular}


AT3G15360 thioredoxin M-type 4

AT5G38430 $\quad$ Ribulose bisphosphate carboxylase (small chain) family protein

AT4G40030 Histone superfamily protein

ATCG00770 ribosomal protein S8

AT2G27710 60S acidic ribosomal protein family

AT5G38410 Ribulose bisphosphate carboxylase (small chain) family protein

AT5G40950 ribosomal protein large subunit 27

AT3G05560 Ribosomal L22e protein family

AT5G61170 Ribosomal protein S19e family protein

AT4G15000 Ribosomal L27e protein family

AT5G54600 Translation protein SH3-like family protein

AT3G04400 Ribosomal protein L14p/L23e family protein

AT2G01720 Ribophorin I

AT3G09500 Ribosomal L29 family protein

AT4G27090 Ribosomal protein L14

ATCG00650 ribosomal protein S18

AT2G19760 profilin 1

ATCG00660 ribosomal protein L20

AT3G24500 multiprotein bridging factor $1 \mathrm{C}$

AT1G69620 ribosomal protein L34

AT4G21280 photosystem II subunit QA

AT4G23670 Polyketide cyclase/dehydrase and lipid transport superfamily prot.

AT3G60770 Ribosomal protein S13/S15

AT2G19740 Ribosomal protein L31e family protein

AT4G34620 small subunit ribosomal protein 16

AT4G34870 rotamase cyclophilin 5

ATCG00750 ribosomal protein S11

AT2G04390 Ribosomal S17 family protein

AT5G14670 ADP-ribosylation factor A1B

AT5G18380 Ribosomal protein S5 domain 2-like superfamily protein

ATCG00900 Ribosomal protein S7p/S5e family protein

AT2G44650 chloroplast chaperonin 10

ATCG00065 ribosomal protein S12A

AT4G39260 cold, circadian rhythm, and RNA binding 1

AT5G52650 RNA binding Plectin/S10 domain-containing protein

AT1G74970 ribosomal protein S9

AT2G39460 ribosomal protein L23AA

AT5G11770 NADH-ubiquinone oxidoreductase $20 \mathrm{kDa}$ subunit

AT4G10300 RmIC-like cupins superfamily protein

AT1G73230 Nascent polypeptide-associated complex NAC

AT5G13410 FKBP-like peptidyl-prolyl cis-trans isomerase family protein

AT3G04920 Ribosomal protein S24e family protein

AT1G70600 Ribosomal protein L18e/L15 superfamily protein

AT4G11010 nucleoside diphosphate kinase 3

AT5G59870 histone $\mathrm{H} 2 \mathrm{~A} 6$

ATCG01120 chloroplast ribosomal protein S15

AT2G34480 Ribosomal protein L18ae/LX family protein

ATCG00780 ribosomal protein L14

AT4G05180 photosystem II subunit Q-2

AT2G20260 photosystem I subunit E-2

AT5G63310 nucleoside diphosphate kinase 2

AT3G62030 rotamase CYP 4

AT5G65350 histone 311

AT3G49910 Translation protein SH3-like family protein

AT3G48930 Nucleic acid-binding, OB-fold-like protein

AT5G13120 cyclophilin 20-2

AT2G16600 rotamase CYP 3

AT3G54210 Ribosomal protein L17 family protein

AT5G08410 ferredoxin/thioredoxin reductase subunit A (variable subunit) 2

AT2G42740 ribosomal protein large subunit 16A

AT1G22780 Ribosomal protein S13/S18 family

AT5G41520 RNA binding Plectin/S10 domain-containing protein

AT5G23820 MD-2-related lipid recognition domain-containing protein

AT2G07707 Plant mitochondrial ATPase, F0 complex, subunit 8 protein

AT5G57290 60S acidic ribosomal protein family

AT2G22170 Lipase/lipooxygenase, PLAT/LH2 family protein

AT1G59860 HSP20-like chaperones superfamily protein

AT5G52840 NADH-ubiquinone oxidoreductase-related

AT3G56340 Ribosomal protein S26e family protein

AT3G52960 Thioredoxin superfamily protein-PRXIIE

AT1G27400 Ribosomal protein L22p/L17e family protein

AT2G30140 UDP-Glycosyltransferase superfamily protein

AT5G27850 Ribosomal protein L18e/L15 superfamily protein

AT4G17560 Ribosomal protein L19 family protein

AT1G15820 light harvesting complex photosystem II subunit 6

AT3G18740 Ribosomal protein L7Ae/L30e/S12e/Gadd45 family protein

AT5G59880 actin depolymerizing factor 3

AT1G11750 CLP protease proteolytic subunit 6

AT1G09590 Translation protein SH3-like family protein

AT1G77940 Ribosomal protein L7Ae/L30e/S12e/Gadd45 family protein

AT3G44100 MD-2-related lipid recognition domain-containing protein

AT1G67700 unknown protein

AT5G23740 ribosomal protein S11-beta

\begin{tabular}{|l|l|}
26 & \\
69 & 7 \\
36 & \\
\hline
\end{tabular}


AT3G47810 Calcineurin-like metallo-phosphoesterase superfamily protein

AT3G60245 Zinc-binding ribosomal protein family protein

AT1G05190 Ribosomal protein L6 family

AT1G13900 Purple acid phosphatases superfamily protein

AT3G21200 proton gradient regulation 7

AT4G30810 serine carboxypeptidase-like 29

AT5G62670 H(+)-ATPase 11

\begin{tabular}{c|c|c|c|c|}
$\mathbf{1 7}$ & 3 & 2.40 & 1.26 & $9.7 \mathrm{E}-03$ \\
\hline 35 & 3 & 2.34 & 1.23 & $9.2 \mathrm{E}-03$ \\
\hline 62 & 20 & 2.33 & 1.22 & $9.9 \mathrm{E}-03$ \\
\hline 5 & 3 & 0.01 & -6.64 & $8.2 \mathrm{E}-17$ \\
\hline $\mathbf{1 2}$ & $\mathbf{3}$ & $\mathbf{0 . 0 1}$ & $\mathbf{- 6 . 6 4}$ & $\mathbf{8 . 2 E}-17$ \\
\hline 4 & 2 & 0.01 & -6.64 & $8.2 \mathrm{E}-17$ \\
$\mathbf{1 2}$ & $\mathbf{2}$ & $\mathbf{0 . 0 1}$ & $\mathbf{- 6 . 6 4}$ & $\mathbf{8 . 2 E}-\mathbf{1 7}$
\end{tabular}

Suppl. Table 4.7. List of proteins that are differentially expressed in amiR23.6 compared to control plants after heat treatment.

\begin{tabular}{|c|c|c|c|c|c|c|}
\hline Accession & Description & $\begin{array}{l}\text { Coverage } \\
{[\%]}\end{array}$ & $\begin{array}{l}\text { \# Unique } \\
\text { Peptides }\end{array}$ & $\begin{array}{l}\text { Fold } \\
\text { change }\end{array}$ & $\begin{array}{c}\text { Fold } \\
\text { change } \\
\left(\log _{2}\right)\end{array}$ & $\begin{array}{l}\text { Adj. P- } \\
\text { Value }\end{array}$ \\
\hline AT1G03860 & prohibitin 2 & 13 & 3 & 100 & 6.64 & $8.4 \mathrm{E}-17$ \\
\hline AT5G64040 & $\begin{array}{l}\text { photosystem I reaction center subunit PSI-N, putative / PSI-N, } \\
\text { putative (PSAN) }\end{array}$ & 11 & 2 & 100 & 6.64 & $8.4 \mathrm{E}-17$ \\
\hline AT5G59720 & heat shock protein 18.2 & 52 & 5 & 7.97 & 2.99 & $9.0 \mathrm{E}-11$ \\
\hline AT4G40030 & Histone superfamily protein & 36 & 2 & 6.54 & 2.71 & $1.2 \mathrm{E}-09$ \\
\hline AT4G34870 & rotamase cyclophilin 5 & 42 & 5 & 5.64 & 2.49 & $4.1 \mathrm{E}-12$ \\
\hline AT4G09320 & Nucleoside diphosphate kinase family protein & 58 & 8 & 4.21 & 2.07 & $7.8 \mathrm{E}-05$ \\
\hline AT3G46230 & heat shock protein 17.4 & 74 & 6 & 4.02 & 2.01 & $1.3 \mathrm{E}-08$ \\
\hline AT5G52650 & RNA binding Plectin/S10 domain-containing protein & 25 & 5 & 3.65 & 1.87 & $3.1 \mathrm{E}-06$ \\
\hline AT5G41520 & RNA binding Plectin/S10 domain-containing protein & 41 & 8 & 3.20 & 1.68 & $4.5 \mathrm{E}-04$ \\
\hline AT4G10250 & HSP20-like chaperones superfamily protein & 32 & 8 & 2.79 & 1.48 & 2.7E-05 \\
\hline AT1G69620 & ribosomal protein L34 & 29 & 2 & 2.76 & 1.47 & $2.6 \mathrm{E}-03$ \\
\hline AT2G29500 & HSP20-like chaperones superfamily protein & 44 & 4 & 2.19 & 1.13 & $1.1 \mathrm{E}-02$ \\
\hline AT1G26480 & general regulatory factor 12 & 19 & 2 & 0.23 & -2.1 & 2.4E-03 \\
\hline AT5G20230 & blue-copper-binding protein & 21 & 3 & 0.16 & -2.62 & $2.5 \mathrm{E}-10$ \\
\hline AT4G25200 & mitochondrion-localized small heat shock protein 23.6 & 59 & 12 & 0.09 & -3.42 & $8.4 \mathrm{E}-17$ \\
\hline AT5G11720 & Glycosyl hydrolases family 31 protein & 4 & 2 & 0.01 & -6.64 & $8.4 \mathrm{E}-17$ \\
\hline AT3G09640 & ascorbate peroxidase 2 & 21 & 3 & 0.01 & -6.64 & $8.4 \mathrm{E}-17$ \\
\hline AT3G54360 & zinc ion binding & 6 & 2 & 0.01 & -6.64 & $8.4 \mathrm{E}-17$ \\
\hline AT4G19006 & Proteasome component $(\mathrm{PCl})$ domain protein & 24 & 2 & 0.01 & -6.64 & $8.4 \mathrm{E}-17$ \\
\hline AT5G47860 & Protein of unknown function (DUF1350) & 15 & 3 & 0.01 & -6.64 & $8.4 \mathrm{E}-17$ \\
\hline AT3G12490 & cystatin B & 15 & 3 & 0.01 & -6.64 & $8.4 \mathrm{E}-17$ \\
\hline AT5G39080 & HXXXD-type acyl-transferase family protein & 8 & 3 & 0.01 & -6.64 & $8.4 \mathrm{E}-17$ \\
\hline AT3G61260 & Remorin family protein & 25 & 3 & 0.01 & -6.64 & $8.4 \mathrm{E}-17$ \\
\hline AT1G22360 & UDP-glucosyl transferase 85A2 & 10 & 3 & 0.01 & -6.64 & $8.4 \mathrm{E}-17$ \\
\hline AT2G18980 & Peroxidase superfamily protein & 24 & 3 & 0.01 & -6.64 & 8.4E-17 \\
\hline
\end{tabular}

Suppl. Table 4.8. List of proteins that are differentially expressed in amiR26.5 compared to control plants after heat treatment.

\begin{tabular}{|c|c|c|c|c|c|c|}
\hline Accession & Description & $\begin{array}{c}\text { Coverage } \\
{[\%]}\end{array}$ & $\begin{array}{l}\text { \# Unique } \\
\text { Peptides }\end{array}$ & $\begin{array}{c}\text { Fold } \\
\text { change }\end{array}$ & $\begin{array}{c}\text { Fold } \\
\text { change } \\
\left(\log _{2}\right)\end{array}$ & $\begin{array}{l}\text { Adj. P- } \\
\text { Value }\end{array}$ \\
\hline AT4G18100 & Ribosomal protein L32e & 31 & 3 & 100 & 6.64 & $1.0 \mathrm{E}-17$ \\
\hline AT1G54050 & HSP20-like chaperones superfamily protein & 54 & 10 & 100 & 6.64 & $1.0 \mathrm{E}-17$ \\
\hline ATCG00820 & ribosomal protein S19 & 40 & 4 & 100 & 6.64 & $1.0 \mathrm{E}-17$ \\
\hline AT3G02080 & Ribosomal protein S19e family protein & 45 & 4 & 100 & 6.64 & 1.0E-17 \\
\hline ATCG00810 & ribosomal protein L22 & 40 & 5 & 100 & 6.64 & $1.0 \mathrm{E}-17$ \\
\hline AT2G27720 & $60 S$ acidic ribosomal protein family & 76 & 3 & 100 & 6.64 & $1.0 \mathrm{E}-17$ \\
\hline AT4G03280 & photosynthetic electron transfer $\mathrm{C}$ & 14 & 2 & 100 & 6.64 & $1.0 \mathrm{E}-17$ \\
\hline AT2G20450 & Ribosomal protein L14 & 44 & 2 & 100 & 6.64 & $1.0 \mathrm{E}-17$ \\
\hline AT4G12800 & photosystem I subunit I & 19 & 3 & 100 & 6.64 & $1.0 \mathrm{E}-17$ \\
\hline AT5G53490 & Tetratricopeptide repeat (TPR)-like superfamily protein & 39 & 9 & 100 & 6.64 & $1.0 \mathrm{E}-17$ \\
\hline AT1G75350 & Ribosomal protein L31 & 32 & 4 & 100 & 6.64 & $1.0 \mathrm{E}-17$ \\
\hline AT3G46000 & actin depolymerizing factor 2 & 24 & 2 & 100 & 6.64 & $1.0 \mathrm{E}-17$ \\
\hline AT5G28060 & Ribosomal protein S24e family protein & 31 & 2 & 100 & 6.64 & $1.0 \mathrm{E}-17$ \\
\hline AT1G29250 & Alba DNA/RNA-binding protein & 37 & 2 & 100 & 6.64 & $1.0 \mathrm{E}-17$ \\
\hline AT3G10520 & haemoglobin 2 & 27 & 4 & 100 & 6.64 & $1.0 \mathrm{E}-17$ \\
\hline AT3G53990 & Adenine nucleotide alpha hydrolases-like superfamily protein & 46 & 5 & 100 & 6.64 & $1.0 \mathrm{E}-17$ \\
\hline AT4G03520 & Thioredoxin superfamily protein & 23 & 3 & 100 & 6.64 & $1.0 \mathrm{E}-17$ \\
\hline AT1G23130 & Polyketide cyclase/dehydrase and lipid transport superfamily prot. & 21 & 3 & 100 & 6.64 & $1.0 \mathrm{E}-17$ \\
\hline AT1G16890 & ubiquitin-conjugating enzyme 36 & 10 & 2 & 100 & 6.64 & $1.0 \mathrm{E}-17$ \\
\hline AT1G20580 & Small nuclear ribonucleoprotein family protein & 13 & 2 & 100 & 6.64 & $1.0 \mathrm{E}-17$ \\
\hline ATCG00670 & plastid-encoded CLP P & 21 & 2 & 100 & 6.64 & $1.0 \mathrm{E}-17$ \\
\hline AT3G07470 & Protein of unknown function, DUF538 & 20 & 3 & 100 & 6.64 & $1.0 \mathrm{E}-17$ \\
\hline AT3G52560 & ubiquitin E2 variant $1 \mathrm{D}-4$ & 32 & 2 & 100 & 6.64 & $1.0 \mathrm{E}-17$ \\
\hline AT1G26550 & FKBP-like peptidyl-prolyl cis-trans isomerase family protein & 15 & 2 & 100 & 6.64 & $1.0 \mathrm{E}-17$ \\
\hline AT1G66240 & homolog of anti-oxidant 1 & 25 & 2 & 100 & 6.64 & $1.0 \mathrm{E}-17$ \\
\hline AT1G11430 & plastid developmental protein DAG, putative & 17 & 2 & 100 & 6.64 & $1.0 \mathrm{E}-17$ \\
\hline AT1G64230 & ubiquitin-conjugating enzyme 28 & 20 & 2 & 100 & 6.64 & $1.0 \mathrm{E}-17$ \\
\hline AT4G28660 & photosystem II reaction center PSB28 protein & 12 & 2 & 100 & 6.64 & $1.0 \mathrm{E}-17$ \\
\hline AT2G34160 & Alba DNA/RNA-binding protein & 15 & 2 & 100 & 6.64 & $1.0 \mathrm{E}-17$ \\
\hline AT1G03600 & photosystem II family protein & 28 & 4 & 100 & 6.64 & $1.0 \mathrm{E}-17$ \\
\hline AT1G21065 & unknown protein & 9 & 2 & 100 & 6.64 & $1.0 \mathrm{E}-17$ \\
\hline
\end{tabular}


AT2G33530 serine carboxypeptidase-like 46

AT5G14910 Heavy metal transport/detoxification superfamily protein

AT3G05590 ribosomal protein L18

AT4G23680 Polyketide cyclase/dehydrase and lipid transport superfamily prot.

AT2G36145 unknown protein

AT5G51110 Transcriptional coactivator/pterin dehydratase

AT1G15930 Ribosomal protein L7Ae/L30e/S12e/Gadd45 family protein

AT4G29350 profilin 2

AT2G01520 MLP-like protein 328

AT3G22230 Ribosomal L27e protein family

AT2G21660 cold, circadian rhythm, and rna binding 2

AT2G21530 SMAD/FHA domain-containing protein

AT1G63970 isoprenoid F

AT3G60370 FKBP-like peptidyl-prolyl cis-trans isomerase family protein

AT2G44920 Tetratricopeptide repeat (TPR)-like superfamily protein

AT3G10090 Nucleic acid-binding, OB-fold-like protein

AT1G08880 Histone superfamily protein

ATCG01060 iron-sulfur cluster binding;electron carriers; 4 iron, 4 sulfur cluster

binding

AT5G64040 photosystem I reaction center subunit PSI-N, chloroplast, putative

AT5G64040 PSI-N, putative (PSAN)

AT2G38540 lipid transfer protein 1

AT1G07820 Histone superfamily protein

AT1G31860 histidine biosynthesis bifunctional protein (HISIE)

AT5G65840 Thioredoxin superfamily protein

AT4G09320 Nucleoside diphosphate kinase family protein

AT3G44890 ribosomal protein L9

AT1G67090 ribulose bisphosphate carboxylase small chain 1A

AT5G38430 Ribulose bisphosphate carboxylase (small chain) family prot.

AT4G28706 pfkB-like carbohydrate kinase family protein

ATCG00770 ribosomal protein S8

AT1G79850 ribosomal protein $\mathrm{S} 17$

AT5G38410 Ribulose bisphosphate carboxylase (small chain) family prot.

AT4G40030 Histone superfamily protein

AT5G40950 ribosomal protein large subunit 27

AT4G15000 Ribosomal L27e protein family

AT3G05560 Ribosomal L22e protein family

AT3G15360 thioredoxin M-type 4

AT2G27710 60S acidic ribosomal protein family

AT5G61170 Ribosomal protein S19e family protein

AT4G23670 Polyketide cyclase/dehydrase and lipid transport superfamily prot.

AT3G09500 Ribosomal L29 family protein

AT3G04400 Ribosomal protein L14p/L23e family protein

AT2G19760 profilin 1

AT2G44650 chloroplast chaperonin 10

ATCG00660 ribosomal protein L20

AT1G69620 ribosomal protein L34

AT2G01720 Ribophorin I

ATCG00750 ribosomal protein S11

ATCG00065 ribosomal protein S12A

AT3G60770 Ribosomal protein S13/S15

AT4G39260 cold, circadian rhythm, and RNA binding

ATCG00900 Ribosomal protein S7p/S5e family protein

AT4G11010 nucleoside diphosphate kinase 3

ATCG00650 ribosomal protein S18

AT4G34620 small subunit ribosomal protein 16

AT1G74970 ribosomal protein S9

AT3G24500 multiprotein bridging factor 1C

AT5G18380 Ribosomal protein S5 domain 2-like superfamily protein

AT4G10300 RmIC-like cupins superfamily protein

AT2G19740 Ribosomal protein L31e family protein

AT2G04390 Ribosomal S17 family protein

AT4G27090 Ribosomal protein L14

ATCG01120 chloroplast ribosomal protein S15

AT5G14670 ADP-ribosylation factor A1B

ATCG00780 ribosomal protein L14

AT5G54600 Translation protein SH3-like family protein

AT4G21280 photosystem II subunit QA AT1G06650 2-oxoglutarate (2OG) and Fe(II)-dependent oxygenase superfamily

AT4G05180 photosystem II subunit Q-2

AT5G63310 nucleoside diphosphate kinase 2

AT5G08410 ferredoxin/thioredoxin reductase subunit A (variable subunit) 2

AT5G57290 60S acidic ribosomal protein family

AT1G73230 Nascent polypeptide-associated complex NAC

AT5G13410 FKBP-like peptidyl-prolyl cis-trans isomerase family protein

AT2G20260 photosystem I subunit E-2

AT3G25920 ribosomal protein L15

AT2G30140 UDP-Glycosyltransferase superfamily protein

AT1G70600 Ribosomal protein L18e/L15 superfamily protein

AT3G54210 Ribosomal protein L17 family protein

AT5G23820 MD-2-related lipid recognition domain-containing protein

AT3G04920 Ribosomal protein S24e family protein

\begin{tabular}{|c|c|c|c|c|}
\hline 4 & 2 & 100 & 6.64 & 1.0E-17 \\
\hline 15 & 2 & 100 & 6.64 & 1.0E-17 \\
\hline 52 & 3 & 100 & 6.64 & $1.0 \mathrm{E}-17$ \\
\hline 21 & 3 & 100 & 6.64 & $1.0 \mathrm{E}-17$ \\
\hline 9 & 2 & 100 & 6.64 & $1.0 \mathrm{E}-17$ \\
\hline 12 & 2 & 100 & 6.64 & $1.0 \mathrm{E}-17$ \\
\hline 42 & 3 & 100 & 6.64 & $1.0 \mathrm{E}-17$ \\
\hline 32 & 3 & 100 & 6.64 & $1.0 \mathrm{E}-17$ \\
\hline 30 & 2 & 100 & 6.64 & $1.0 \mathrm{E}-17$ \\
\hline 34 & 2 & 100 & 6.64 & $1.0 \mathrm{E}-17$ \\
\hline 44 & 4 & 100 & 6.64 & $1.0 \mathrm{E}-17$ \\
\hline 12 & 2 & 100 & 6.64 & $1.0 \mathrm{E}-17$ \\
\hline 17 & 3 & 100 & 6.64 & $1.0 \mathrm{E}-17$ \\
\hline 14 & 3 & 100 & 6.64 & $1.0 \mathrm{E}-17$ \\
\hline 36 & 5 & 100 & 6.64 & $1.0 \mathrm{E}-17$ \\
\hline 23 & 2 & 100 & 6.64 & $1.0 \mathrm{E}-17$ \\
\hline 45 & 2 & 100 & 6.64 & 1.0E-17 \\
\hline 27 & 2 & 100 & 6.64 & $1.0 \mathrm{E}-17$ \\
\hline 11 & 2 & 100 & 6.64 & $1.0 \mathrm{E}-17$ \\
\hline 22 & 2 & 100 & 6.64 & $1.0 \mathrm{E}-17$ \\
\hline 50 & 8 & 92.09 & 6.53 & $1.0 \mathrm{E}-17$ \\
\hline 22 & 3 & 85.75 & 6.42 & $1.0 \mathrm{E}-17$ \\
\hline 8 & 2 & 79.80 & 6.32 & $1.0 \mathrm{E}-17$ \\
\hline 58 & 8 & 77.28 & 6.27 & $1.0 \mathrm{E}-17$ \\
\hline 47 & 10 & 57.05 & 5.83 & $1.0 \mathrm{E}-17$ \\
\hline 64 & 15 & 39.07 & 5.29 & $1.0 \mathrm{E}-17$ \\
\hline 69 & 7 & 32.15 & 5.01 & $1.0 \mathrm{E}-17$ \\
\hline 17 & 6 & 31.28 & 4.97 & $1.0 \mathrm{E}-17$ \\
\hline 63 & 10 & 28.76 & 4.85 & $1.0 \mathrm{E}-17$ \\
\hline 31 & 4 & 28.47 & 4.83 & $1.0 \mathrm{E}-17$ \\
\hline 69 & 7 & 26.75 & 4.74 & $1.0 \mathrm{E}-17$ \\
\hline 36 & 2 & 24.65 & 4.62 & $1.0 \mathrm{E}-17$ \\
\hline 23 & 4 & 24.20 & 4.6 & $1.0 \mathrm{E}-17$ \\
\hline 43 & 4 & 23.47 & 4.55 & $1.0 \mathrm{E}-17$ \\
\hline 64 & 2 & 22.37 & 4.48 & $1.0 \mathrm{E}-17$ \\
\hline 26 & 4 & 21.41 & 4.42 & $1.0 \mathrm{E}-17$ \\
\hline 68 & 3 & 18.90 & 4.24 & $1.0 \mathrm{E}-17$ \\
\hline 48 & 5 & 18.50 & 4.21 & 1.0E-17 \\
\hline 64 & 9 & 18.33 & 4.2 & $1.0 \mathrm{E}-17$ \\
\hline 31 & 5 & 17.82 & 4.16 & $1.0 \mathrm{E}-17$ \\
\hline 48 & 7 & 16.41 & 4.04 & $1.0 \mathrm{E}-17$ \\
\hline 43 & 4 & 16.27 & 4.02 & $1.0 \mathrm{E}-17$ \\
\hline 37 & 4 & 14.38 & 3.85 & $1.0 \mathrm{E}-17$ \\
\hline 45 & 6 & 14.11 & 3.82 & $1.0 \mathrm{E}-17$ \\
\hline 29 & 2 & 14.09 & 3.82 & $1.0 \mathrm{E}-17$ \\
\hline 8 & 2 & 11.63 & 3.54 & $1.3 \mathrm{E}-15$ \\
\hline 28 & 5 & 11.52 & 3.53 & $1.0 \mathrm{E}-17$ \\
\hline 31 & 2 & 11.32 & 3.5 & $2.2 \mathrm{E}-16$ \\
\hline 46 & 9 & 11.24 & 3.49 & $1.0 \mathrm{E}-17$ \\
\hline 51 & 5 & 11.13 & 3.48 & $1.0 \mathrm{E}-17$ \\
\hline 46 & 8 & 10.59 & 3.4 & $1.0 \mathrm{E}-17$ \\
\hline 31 & 6 & 10.44 & 3.38 & $1.0 \mathrm{E}-17$ \\
\hline 33 & 3 & 9.70 & 3.28 & $1.0 \mathrm{E}-17$ \\
\hline 68 & 6 & 9.55 & 3.26 & 1.0E-17 \\
\hline 31 & 6 & 8.95 & 3.16 & $1.0 \mathrm{E}-17$ \\
\hline 33 & 4 & 8.94 & 3.16 & $1.0 \mathrm{E}-17$ \\
\hline 50 & 2 & 8.92 & 3.16 & $1.0 \mathrm{E}-17$ \\
\hline 22 & 3 & 8.27 & 3.05 & $1.8 \mathrm{E}-14$ \\
\hline 8 & 2 & 8.22 & 3.04 & $1.0 \mathrm{E}-17$ \\
\hline 30 & 5 & 8.05 & 3.01 & $1.0 \mathrm{E}-17$ \\
\hline 56 & 6 & 7.64 & 2.93 & $1.0 \mathrm{E}-17$ \\
\hline 47 & 5 & 7.59 & 2.92 & $1.8 \mathrm{E}-15$ \\
\hline 63 & 13 & 7.19 & 2.85 & $1.0 \mathrm{E}-17$ \\
\hline 52 & 5 & 7.00 & 2.81 & $1.0 \mathrm{E}-17$ \\
\hline 28 & 6 & 6.36 & 2.67 & 1.3E-13 \\
\hline 52 & 15 & 6.35 & 2.67 & $1.0 \mathrm{E}-17$ \\
\hline 19 & 5 & 6.20 & 2.63 & $5.4 \mathrm{E}-07$ \\
\hline 46 & 10 & 6.08 & 2.6 & $1.2 \mathrm{E}-12$ \\
\hline 43 & 9 & 5.96 & 2.57 & $1.0 \mathrm{E}-17$ \\
\hline 8 & 2 & 5.84 & 2.55 & 3.7E-09 \\
\hline 22 & 2 & 5.74 & 2.52 & 1.5E-09 \\
\hline 41 & 3 & 5.59 & 2.48 & $4.6 \mathrm{E}-08$ \\
\hline 29 & 5 & 5.40 & 2.43 & 2.0E-08 \\
\hline 46 & 2 & 5.34 & 2.42 & 3.2E-12 \\
\hline 36 & 10 & 5.10 & 2.35 & 1.0E-17 \\
\hline 6 & 2 & 5.03 & 2.33 & 1.0E-07 \\
\hline 43 & 6 & 4.95 & 2.31 & 4.6E-11 \\
\hline 20 & 4 & 4.91 & 2.3 & $9.1 \mathrm{E}-12$ \\
\hline 20 & 5 & 4.91 & 2.29 & 4.4E-16 \\
\hline 31 & 3 & 4.89 & 2.29 & $2.6 \mathrm{E}-12$ \\
\hline
\end{tabular}




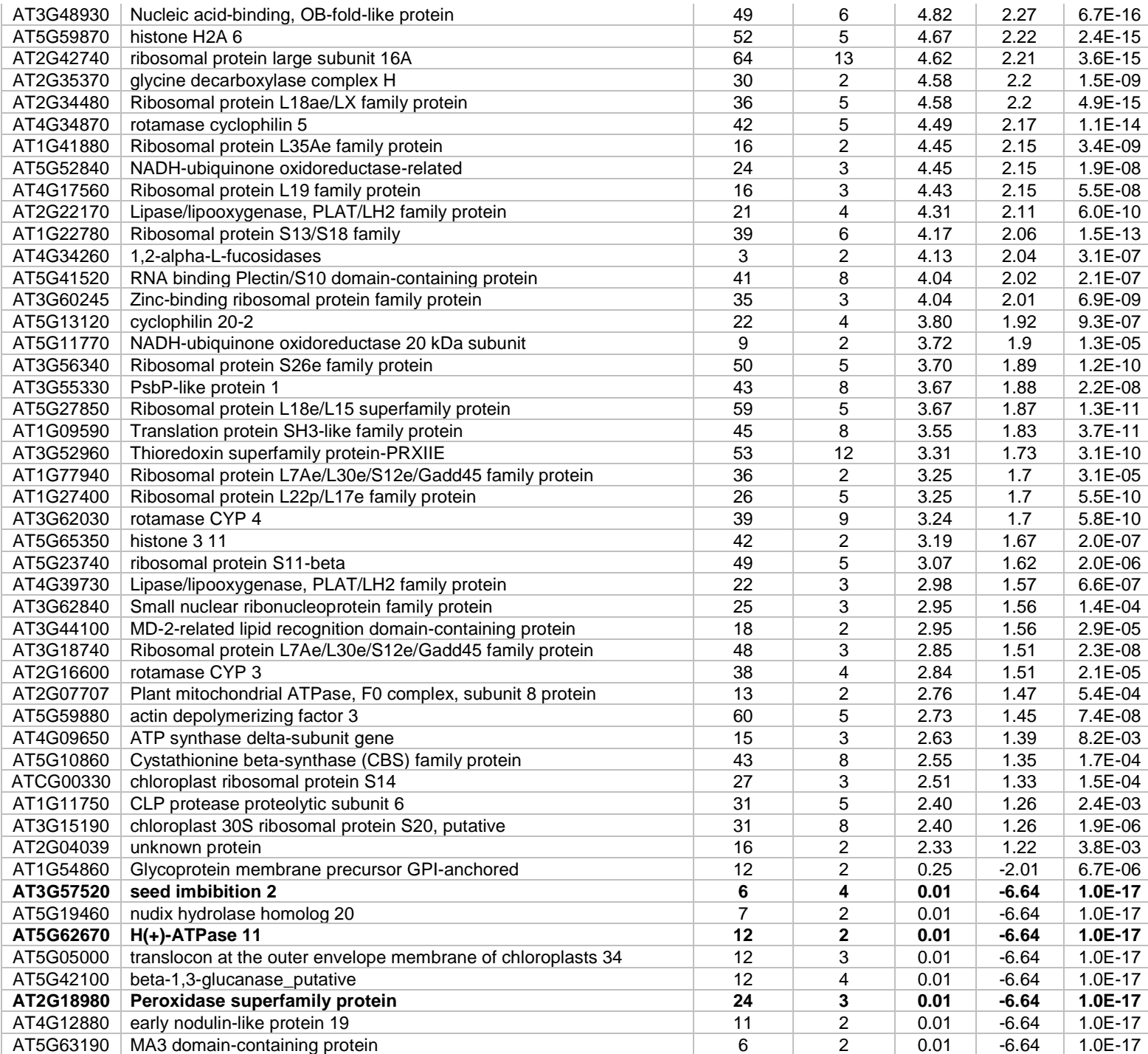

\section{Suppl. Table 4.9. List of proteins that are differentially expressed in amiR23.5/23.6 compared to} control plants after heat treatment.

\begin{tabular}{|c|c|c|c|c|c|c|}
\hline Accession & Description & $\begin{array}{c}\text { Coverage } \\
{[\%]}\end{array}$ & $\begin{array}{l}\text { \# Unique } \\
\text { Peptides }\end{array}$ & $\begin{array}{c}\text { Fold } \\
\text { change }\end{array}$ & $\begin{array}{c}\text { Fold } \\
\text { change } \\
\left(\log _{2}\right)\end{array}$ & $\begin{array}{l}\text { Adj. P- } \\
\text { Value }\end{array}$ \\
\hline AT4G18100 & Ribosomal protein L32e & 31 & 3 & 100 & 6.64 & 7.7E-17 \\
\hline AT1G54050 & HSP20-like chaperones superfamily protein-HSP17.4B & 54 & 10 & 100 & 6.64 & 7.7E-17 \\
\hline AT3G56290 & unknown protein & 17 & 2 & 100 & 6.64 & 7.7E-17 \\
\hline AT5G35680 & Nucleic acid-binding, OB-fold-like protein & 25 & 3 & 100 & 6.64 & 7.7E-17 \\
\hline ATCG00820 & ribosomal protein S19 & 40 & 4 & 100 & 6.64 & 7.7E-17 \\
\hline AT1G65220 & ARM repeat superfamily protein & 13 & 2 & 100 & 6.64 & 7.7E-17 \\
\hline AT3G02080 & Ribosomal protein $\mathrm{S} 19 \mathrm{e}$ family protein & 45 & 4 & 100 & 6.64 & 7.7E-17 \\
\hline ATCG00810 & ribosomal protein L22 & 40 & 5 & 100 & 6.64 & 7.7E-17 \\
\hline AT1G16700 & Alpha-helical ferredoxin & 7 & 2 & 100 & 6.64 & 7.7E-17 \\
\hline AT1G03860 & prohibitin 2 & 13 & 3 & 100 & 6.64 & 7.7E-17 \\
\hline AT2G27720 & $60 S$ acidic ribosomal protein family & 76 & 3 & 100 & 6.64 & 7.7E-17 \\
\hline AT4G03280 & photosynthetic electron transfer $\mathrm{C}$ & 14 & 2 & 100 & 6.64 & 7.7E-17 \\
\hline AT2G20450 & Ribosomal protein L14 & 44 & 2 & 100 & 6.64 & 7.7E-17 \\
\hline AT4G12800 & photosystem I subunit I & 19 & 3 & 100 & 6.64 & 7.7E-17 \\
\hline AT5G53490 & Tetratricopeptide repeat (TPR)-like superfamily protein & 39 & 9 & 100 & 6.64 & 7.7E-17 \\
\hline AT3G05590 & ribosomal protein L18 & 52 & 3 & 100 & 6.64 & 7.7E-17 \\
\hline AT3G46000 & actin depolymerizing factor 2 & 24 & 2 & 100 & 6.64 & 7.7E-17 \\
\hline AT5G28060 & Ribosomal protein S24e family protein & 31 & 2 & 100 & 6.64 & 7.7E-17 \\
\hline AT4G03520 & Thioredoxin superfamily protein & 23 & 3 & 100 & 6.64 & 7.7E-17 \\
\hline AT3G10520 & haemoglobin 2 & 27 & 4 & 100 & 6.64 & 7.7E-17 \\
\hline AT1G29250 & Alba DNA/RNA-binding protein & 37 & 2 & 100 & 6.64 & 7.7E-17 \\
\hline AT3G53990 & Adenine nucleotide alpha hydrolases-like superfamily protein & 46 & 5 & 100 & 6.64 & 7.7E-17 \\
\hline AT1G17880 & basic transcription factor 3 & 28 & 2 & 100 & 6.64 & 7.7E-17 \\
\hline AT5G23010 & methylthioalkylmalate synthase 1 & 7 & 3 & 100 & 6.64 & 7.7E-17 \\
\hline AT1G20580 & Small nuclear ribonucleoprotein family protein & 13 & 2 & 100 & 6.64 & 7.7E-17 \\
\hline
\end{tabular}


AT3G07470 Protein of unknown function, DUF538

ATCG00670 plastid-encoded CLP P

AT3G52560 ubiquitin E2 variant 1D-4

AT1G26550 FKBP-like peptidyl-prolyl cis-trans isomerase family protein

AT4G28660 photosystem II reaction center PSB28 protein

AT1G75350 Ribosomal protein L31

AT1G76450 Photosystem II reaction center PsbP family protein

AT4G23680 Polyketide cyclase/dehydrase and lipid transport superfamily prot.

AT2G36145 unknown protein

AT1G23130 Polyketide cyclase/dehydrase and lipid transport superfamily prot.

AT3G25220 FK506-binding protein $15 \mathrm{kD}-1$

AT5G64040 photosystem I reaction center subunit PSI-N, putative / PSI-N, putative (PSAN)

AT1G11430 plastid developmental protein DAG, putative

AT1G64230 ubiquitin-conjugating enzyme 28

AT2G34160 Alba DNA/RNA-binding protein

AT1G08880 Histone superfamily protein

AT1G03600 photosystem II family protein

AT1G21065 unknown protein

AT4G11600 glutathione peroxidase 6-GPX6

AT2G44920 Tetratricopeptide repeat (TPR)-like superfamily protein

AT1G63970 isoprenoid $F$

AT3G22230 Ribosomal L27e protein family

AT2G01520 MLP-like protein 328

AT3G10090 Nucleic acid-binding, OB-fold-like protein

AT3G60370 FKBP-like peptidyl-prolyl cis-trans isomerase family protein

AT4G29350 profilin 2

AT2G21660 cold, circadian rhythm, and rna binding 2

AT2G06050 oxophytodienoate-reductase 3

AT2G21530 SMAD/FHA domain-containing protein

AT1G15930 Ribosomal protein L7Ae/L30e/S12e/Gadd45 family protein

AT3G59540 Ribosomal L38e protein family

AT5G12020 $17.6 \mathrm{kDa}$ class II heat shock protein

AT4G09320 Nucleoside diphosphate kinase family protein

AT1G07820 Histone superfamily protein

AT1G31860 histidine biosynthesis bifunctional protein (HISIE)

AT3G44890 ribosomal protein L9

AT5G65840 Thioredoxin superfamily protein

AT5G38430 Ribulose bisphosphate carboxylase family protein

AT3G15360 thioredoxin M-type 4

AT5G40950 ribosomal protein large subunit 27

AT1G79850 ribosomal protein S17

AT4G40030 Histone superfamily protein

AT3G05560 Ribosomal L22e protein family

AT2G27710 60S acidic ribosomal protein family

AT5G38410 Ribulose bisphosphate carboxylase family protein

AT1G67090 ribulose bisphosphate carboxylase small chain 1A

AT4G15000 Ribosomal L27e protein family

AT3G04400 Ribosomal protein L14p/L23e family protein

ATCG00770 ribosomal protein S8

AT5G61170 Ribosomal protein S19e family protein

AT4G23670 Polyketide cyclase/dehydrase and lipid transport superfamily prot.

AT5G54600 Translation protein SH3-like family protein

AT3G09500 Ribosomal L29 family protein

AT5G18380 Ribosomal protein S5 domain 2-like superfamily protein

AT3G60770 Ribosomal protein S13/S15

AT2G01720 Ribophorin I

AT1G69620 ribosomal protein L34

AT3G24500 multiprotein bridging factor 1C

ATCG00660 ribosomal protein L20

AT4G34870 rotamase cyclophilin 5

AT5G59720 heat shock protein 18.2

AT4G39260 cold, circadian rhythm, and RNA binding 1

AT2G19760 profilin 1

ATCG00900 Ribosomal protein S7p/S5e family protein

ATCG00065 ribosomal protein S12A

AT5G52650 RNA binding Plectin/S10 domain-containing protein

AT2G04390 Ribosomal S17 family protein

AT2G44650 chloroplast chaperonin 10

ATCG00750 ribosomal protein S11

AT2G19740 Ribosomal protein L31e family protein

AT1G74970 ribosomal protein S9

AT1G73230 Nascent polypeptide-associated complex NAC

AT4G11010 nucleoside diphosphate kinase 3

ATCG00650 ribosomal protein S18

AT5G14670 ADP-ribosylation factor A1B

AT5G57290 60S acidic ribosomal protein family

AT4G21280 photosystem II subunit QA

AT2G39460 ribosomal protein L23AA

AT4G34620 small subunit ribosomal protein 16

ATCG01120 chloroplast ribosomal protein S15

AT3G04920 Ribosomal protein S24e family protein

AT1G70600 Ribosomal protein L18e/L15 superfamily protein

\begin{tabular}{|c|c|c|c|c|}
\hline 20 & 3 & 100 & 6.64 & 7.7E-17 \\
\hline 21 & 2 & 100 & 6.64 & 7.7E-17 \\
\hline 32 & 2 & 100 & 6.64 & 7.7E-17 \\
\hline 15 & 2 & 100 & 6.64 & 7.7E-17 \\
\hline 12 & 2 & 100 & 6.64 & 7.7E-17 \\
\hline 32 & 4 & 100 & 6.64 & 7.7E-17 \\
\hline 36 & 5 & 100 & 6.64 & 7.7E-17 \\
\hline 21 & 3 & 100 & 6.64 & 7.7E-17 \\
\hline 9 & 2 & 100 & 6.64 & 7.7E-17 \\
\hline 21 & 3 & 100 & 6.64 & 7.7E-17 \\
\hline 16 & 2 & 100 & 6.64 & 7.7E-17 \\
\hline 11 & 2 & 100 & 6.64 & 7.7E-17 \\
\hline 17 & 2 & 100 & 6.64 & 7.7E-17 \\
\hline 20 & 2 & 100 & 6.64 & 7.7E-17 \\
\hline 15 & 2 & 100 & 6.64 & 7.7E-17 \\
\hline 45 & 2 & 100 & 6.64 & 7.7E-17 \\
\hline 28 & 4 & 100 & 6.64 & 7.7E-17 \\
\hline 9 & 2 & 100 & 6.64 & 7.7E-17 \\
\hline 22 & 4 & 100 & 6.64 & 7.7E-17 \\
\hline 36 & 5 & 100 & 6.64 & 7.7E-17 \\
\hline 17 & 3 & 100 & 6.64 & 7.7E-17 \\
\hline 34 & 2 & 100 & 6.64 & 7.7E-17 \\
\hline 30 & 2 & 100 & 6.64 & 7.7E-17 \\
\hline 23 & 2 & 100 & 6.64 & $7.7 \mathrm{E}-17$ \\
\hline 14 & 3 & 100 & 6.64 & 7.7E-17 \\
\hline 32 & 3 & 100 & 6.64 & 7.7E-17 \\
\hline 44 & 4 & 100 & 6.64 & 7.7E-17 \\
\hline 6 & 2 & 100 & 6.64 & 7.7E-17 \\
\hline 12 & 2 & 100 & 6.64 & 7.7E-17 \\
\hline 42 & 3 & 100 & 6.64 & 7.7E-17 \\
\hline 35 & 2 & 100 & 6.64 & 7.7E-17 \\
\hline 41 & 3 & 100 & 6.64 & 7.7E-17 \\
\hline 58 & 8 & 75.18 & 6.23 & 7.7E-17 \\
\hline 50 & 8 & 73.00 & 6.19 & 7.7E-17 \\
\hline 22 & 3 & 56.40 & 5.82 & $7.7 \mathrm{E}-17$ \\
\hline 47 & 10 & 44.89 & 5.49 & 7.7E-17 \\
\hline 8 & 2 & 43.13 & 5.43 & 7.7E-17 \\
\hline 69 & 7 & 35.68 & 5.16 & 7.7E-17 \\
\hline 26 & 4 & 28.15 & 4.82 & 7.7E-17 \\
\hline 23 & 4 & 25.65 & 4.68 & 7.7E-17 \\
\hline 31 & 4 & 24.79 & 4.63 & $7.7 \mathrm{E}-17$ \\
\hline 36 & 2 & 23.64 & 4.56 & 7.7E-17 \\
\hline 64 & 2 & 22.38 & 4.48 & 7.7E-17 \\
\hline 68 & 3 & 21.73 & 4.44 & 7.7E-17 \\
\hline 69 & 7 & 21.09 & 4.4 & 7.7E-17 \\
\hline 64 & 15 & 20.91 & 4.39 & 7.7E-17 \\
\hline 43 & 4 & 19.85 & 4.31 & 7.7E-17 \\
\hline 48 & 7 & 19.26 & 4.27 & $7.7 \mathrm{E}-17$ \\
\hline 63 & 10 & 18.89 & 4.24 & 7.7E-17 \\
\hline 48 & 5 & 18.77 & 4.23 & 7.7E-17 \\
\hline 64 & 9 & 14.22 & 3.83 & $7.7 \mathrm{E}-17$ \\
\hline 28 & 6 & 13.28 & 3.73 & 7.7E-17 \\
\hline 31 & 5 & 13.00 & 3.7 & 7.7E-17 \\
\hline 50 & 2 & 12.40 & 3.63 & 1.0E-14 \\
\hline 46 & 9 & 12.22 & 3.61 & 7.7E-17 \\
\hline 8 & 2 & 12.18 & 3.61 & $9.0 \mathrm{E}-12$ \\
\hline 29 & 2 & 11.65 & 3.54 & 7.7E-17 \\
\hline 33 & 4 & 11.18 & 3.48 & $5.6 \mathrm{E}-14$ \\
\hline 45 & 6 & 10.78 & 3.43 & $3.8 \mathrm{E}-13$ \\
\hline 42 & 5 & 10.01 & 3.32 & 7.7E-17 \\
\hline 52 & 5 & 10.01 & 3.32 & $8.5 \mathrm{E}-13$ \\
\hline 51 & 5 & 9.94 & 3.31 & $1.9 \mathrm{E}-12$ \\
\hline 43 & 4 & 9.82 & 3.3 & $3.2 \mathrm{E}-10$ \\
\hline 46 & 8 & 9.16 & 3.2 & $3.4 \mathrm{E}-15$ \\
\hline 31 & 2 & 8.80 & 3.14 & $9.0 \mathrm{E}-10$ \\
\hline 25 & 5 & 8.42 & 3.07 & $1.9 \mathrm{E}-10$ \\
\hline 30 & 5 & 8.39 & 3.07 & $1.1 \mathrm{E}-10$ \\
\hline 37 & 4 & 8.39 & 3.07 & 1.3E-09 \\
\hline 28 & 5 & 8.38 & 3.07 & 8.6E-11 \\
\hline 8 & 2 & 7.93 & 2.99 & $6.4 \mathrm{E}-10$ \\
\hline 31 & 6 & 7.87 & 2.98 & 7.0E-11 \\
\hline 41 & 3 & 7.57 & 2.92 & 2.0E-07 \\
\hline 31 & 6 & 7.49 & 2.91 & $6.4 \mathrm{E}-10$ \\
\hline 33 & 3 & 7.46 & 2.9 & 1.0E-09 \\
\hline 63 & 13 & 7.41 & 2.89 & $5.8 \mathrm{E}-10$ \\
\hline 22 & 2 & 7.16 & 2.84 & $4.9 \mathrm{E}-08$ \\
\hline 52 & 15 & 6.81 & 2.77 & $1.4 \mathrm{E}-11$ \\
\hline 42 & 11 & 6.61 & 2.72 & $2.9 \mathrm{E}-11$ \\
\hline 68 & 6 & 6.42 & 2.68 & 1.9E-08 \\
\hline 47 & 5 & 6.36 & 2.67 & 2.5E-07 \\
\hline 31 & 3 & 6.25 & 2.64 & $3.0 \mathrm{E}-08$ \\
\hline 43 & 6 & 6.17 & 2.62 & $3.1 \mathrm{E}-08$ \\
\hline
\end{tabular}


AT4G10300 RmIC-like cupins superfamily protein

AT5G08410 ferredoxin/thioredoxin reductase subunit A (variable subunit) 2

AT5G63310 nucleoside diphosphate kinase 2

AT4G27090 Ribosomal protein L14

AT4G05180 photosystem II subunit Q-2

AT2G42740 ribosomal protein large subunit 16A

AT2G34480 Ribosomal protein L18ae/LX family protein

AT3G49910 Translation protein SH3-like family protein

AT3G46230 heat shock protein 17.4

AT3G48930 Nucleic acid-binding, OB-fold-like protein

AT1G78370 glutathione S-transferase TAU 20

AT5G59870 histone H2A 6

AT5G13120 cyclophilin 20-2

AT5G52840 NADH-ubiquinone oxidoreductase-related

ATCG00780 ribosomal protein L14

AT5G13410 FKBP-like peptidyl-prolyl cis-trans isomerase family protein

AT1G22780 Ribosomal protein S13/S18 family

AT1G27400 Ribosomal protein L22p/L17e family protein

AT2G16600 rotamase CYP 3

AT5G41520 RNA binding Plectin/S10 domain-containing protein

AT2G35370 glycine decarboxylase complex $\mathrm{H}$

AT2G20260 photosystem I subunit E-2

AT3G62030 rotamase CYP 4

AT2G29500 HSP20-like chaperones superfamily protein-HSP17.6B

AT2G07707 Plant mitochondrial ATPase, F0 complex, subunit 8 protein

AT5G23820 MD-2-related lipid recognition domain-containing protein

AT1G77940 Ribosomal protein L7Ae/L30e/S12e/Gadd45 family protein

AT1G07400 HSP20-like chaperones superfamily protein-HSP17.8

AT5G27850 Ribosomal protein L18e/L15 superfamily protein

AT3G25920 ribosomal protein L15

AT5G65350 histone 311

AT3G52960 Thioredoxin superfamily protein-PRXIIE

AT4G17560 Ribosomal protein L19 family protein

AT3G54210 Ribosomal protein L17 family protein

AT1G61520 photosystem I light harvesting complex gene 3

AT3G56340 Ribosomal protein S26e family protein

AT5G59880 actin depolymerizing factor 3

AT1G15820 light harvesting complex photosystem II subunit 6

AT3G62840 Small nuclear ribonucleoprotein family protein

AT5G27670 histone H2A 7

AT3G60245 Zinc-binding ribosomal protein family protein

AT5G23740 ribosomal protein S11-beta

AT3G44100 MD-2-related lipid recognition domain-containing protein

AT3G08740 elongation factor P (EF-P) family protein

AT1G09590 Translation protein SH3-like family protein

AT1G67700 unknown protein

AT1G05190 Ribosomal protein L6 family

AT1G32470 Single hybrid motif superfamily protein

AT3G11940 ribosomal protein 5A

AT5G20250 Raffinose synthase family protein

AT4G15530 pyruvate orthophosphate dikinase

AT5G49360 beta-xylosidase 1

AT5G64100 Peroxidase superfamily protein

AT1G29940 nuclear RNA polymerase A2

AT4G34030 3-methylcrotonyl-CoA carboxylase

AT3G09220 laccase 7

AT3G13750 beta galactosidase 1

AT1G54860 Glycoprotein membrane precursor GPI-anchored

AT1G33610 Leucine-rich repeat (LRR) family protein

AT3G57520 seed imbibition 2

AT4G30810 serine carboxypeptidase-like 29

AT5G62670 $\mathrm{H}(+)$-ATPase 11

AT3G54360 zinc ion binding

AT2G18980 Peroxidase superfamily protein

22
8
43
56
46
6
36
41
74
49

\begin{tabular}{|c|c|}
\hline 22 & \\
\hline 8 & \\
\hline 43 & \\
\hline 56 & \\
\hline 46 & \\
\hline 64 & \\
\hline 36 & \\
\hline 41 & \\
\hline 74 & \\
\hline 49 & \\
\hline 38 & \\
\hline 52 & \\
\hline
\end{tabular}

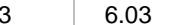

$1.2 \mathrm{E}-08$

3.5E-08

2.7E-07

$4.8 \mathrm{E}-08$

$1.2 \mathrm{E}-07$

9.4E-06

$6.0 \mathrm{E}-06$

$8.5 \mathrm{E}-05$

5.3E-05

1.3E-05

1.1E-04

6.7E-07

8.2E-07

2.3E-05

1.4E-04

3.3E-05

$1.2 \mathrm{E}-05$

3.8E-06

2.1E-04

1.2E-03

2.2E-04

6.2E-04

1.3E-05

$1.8 \mathrm{E}-05$

2.3E-04

5.0E-04

3.4E-05

1.5E-03

2.2E-04

2.1E-02

2.7E-03

3.7E-04

1.7E-02

9.1E-03

1.4E-02

5.1E-03

$5.6 \mathrm{E}-03$

1.1E-02

1.7E-02

9.8E-03

1.1E-02

4.0E-03

4.6E-02

9.9E-03

3.8E-02

2.4E-02

$6.8 \mathrm{E}-03$

6.3E-03

2.8E-03

3.8E-02

4.8E-03

1.2E-03

4.0E-04

4.1E-03

2.0E-04

7.7E-17

7.7E-17

7.7E-17

7.7E-17

Suppl. Table 4.10. List of proteins that are differentially expressed in amiR23.5/23.6/26.5 compared to control plants after heat treatment.

\begin{tabular}{|c|c|c|c|c|c|c|}
\hline Accession & Description & $\begin{array}{c}\text { Coverage } \\
{[\%]}\end{array}$ & $\begin{array}{l}\text { \# Unique } \\
\text { Peptides }\end{array}$ & $\begin{array}{c}\text { Fold } \\
\text { change }\end{array}$ & $\begin{array}{c}\text { Fold } \\
\text { change } \\
\left(\log _{2}\right)\end{array}$ & $\begin{array}{l}\text { Adj. P- } \\
\text { Value }\end{array}$ \\
\hline AT4G18100 & Ribosomal protein L32e & 31 & 3 & 100 & 6.64 & $7.4 \mathrm{E}-17$ \\
\hline AT1G54050 & HSP20-like chaperones superfamily protein & 54 & 10 & 100 & 6.64 & 7.4E-17 \\
\hline AT5G35680 & Nucleic acid-binding, OB-fold-like protein & 25 & 3 & 100 & 6.64 & $7.4 \mathrm{E}-17$ \\
\hline ATCG00820 & ribosomal protein S19 & 40 & 4 & 100 & 6.64 & $7.4 \mathrm{E}-17$ \\
\hline AT3G02080 & Ribosomal protein S19e family protein & 45 & 4 & 100 & 6.64 & $7.4 \mathrm{E}-17$ \\
\hline ATCG00810 & ribosomal protein L22 & 40 & 5 & 100 & 6.64 & $7.4 \mathrm{E}-17$ \\
\hline AT1G03860 & prohibitin 2 & 13 & 3 & 100 & 6.64 & $7.4 \mathrm{E}-17$ \\
\hline AT2G27720 & $60 S$ acidic ribosomal protein family & 76 & 3 & 100 & 6.64 & $7.4 \mathrm{E}-17$ \\
\hline AT4G03280 & photosynthetic electron transfer C & 14 & 2 & 100 & 6.64 & $7.4 \mathrm{E}-17$ \\
\hline AT2G20450 & Ribosomal protein L14 & 44 & 2 & 100 & 6.64 & 7.4E-17 \\
\hline
\end{tabular}


AT4G12800 photosystem I subunit I

\begin{tabular}{|l|r} 
& 19 \\
\hline
\end{tabular}

AT3G53490 Tetratricopeptide repeat (TPR)-like superfamily protein

AT3G53990 Adenine nucleotide alpha hydrolases-like superfamily protein

AT1G29250 Alba DNA/RNA-binding protein

AT1G17880 basic transcription factor 3

AT1G20330 sterol methyltransferase 2

AT3G07470 Protein of unknown function, DUF538

AT2G35810 unknown protein

AT3G52560 ubiquitin E2 variant 1D-4

AT1G20580 Small nuclear ribonucleoprotein family protein

AT1G26550 FKBP-like peptidyl-prolyl cis-trans isomerase family protein

AT1G23130 Polyketide cyclase/dehydrase and lipid transport superfamily prot.

AT5G28060 Ribosomal protein S24e family protein

AT3G05590 ribosomal protein L18

ATCG00670 plastid-encoded CLP P

AT4G23680 Polyketide cyclase/dehydrase and lipid transport superfamily prot.

AT2G36145 unknown protein

AT1G11430 plastid developmental protein DAG, putative

AT2G34160 Alba DNA/RNA-binding protein

AT5G64040 photosystem I reaction center subunit PSI-N, chloroplast,

\begin{tabular}{|l|l} 
& putative / PSI-N, putative (PSAN) \\
\hline AT1G03600 & photosystem II family protein
\end{tabular}

AT4G28660 photosystem II reaction center PSB28 protein

AT1G08880 Histone superfamily protein

AT1G15930 Ribosomal protein L7Ae/L30e/S12e/Gadd45 family protein

ATCG01060 iron-sulfur cluster binding;electron carriers;4 iron, 4 sulfur cluster

binding

AT2G21530 SMAD/FHA domain-containing protein

AT3G10090 Nucleic acid-binding, OB-fold-like protein

AT3G22230 Ribosomal L27e protein family

AT4G29350 profilin 2

AT1G07820 Histone superfamily protein

AT4G09320 Nucleoside diphosphate kinase family protein

AT3G44890 ribosomal protein L9

AT1G31860 histidine biosynthesis bifunctional protein (HISIE)

AT5G65840 Thioredoxin superfamily protein

AT4G40030 Histone superfamily protein

AT1G79850 ribosomal protein S17

\begin{tabular}{|l|l|l|}
\hline AT3G04400 & Ribosomal protein L14p/L23e family protein \\
\hline
\end{tabular}

AT3G05560 Ribosomal L22e protein family

AT5G40950 ribosomal protein large subunit 27

AT2G44050 6,7-dimethyl-8-ribityllumazine synthase / DMRL synthase / lumazine

synthase / riboflavin synthase

AT5G61170 Ribosomal protein S19e family protein

AT4G15000 Ribosomal L27e protein family

AT2G27710 60S acidic ribosomal protein family

AT3G15360 thioredoxin M-type 4

AT3G09500 Ribosomal L29 family protein

AT5G38430 Ribulose bisphosphate carboxylase family protein

AT1G69620 ribosomal protein L34

AT3G60770 Ribosomal protein S13/S15

AT2G01720 Ribophorin I

ATCG00065 ribosomal protein S12A

AT2G19760 profilin 1

AT2G19740 Ribosomal protein L31e family protein

ATCG00660 ribosomal protein L20

AT5G18380 Ribosomal protein S5 domain 2-like superfamily protein

AT4G39260 cold, circadian rhythm, and RNA binding 1

AT5G38410 Ribulose bisphosphate carboxylase family protein

ATCG00750 ribosomal protein S11

AT2G44650 chloroplast chaperonin 10

AT1G67090 ribulose bisphosphate carboxylase small chain 1A

ATCG00650 ribosomal protein S18

AT3G24500 multiprotein bridging factor $1 \mathrm{C}$

AT4G17560 Ribosomal protein L19 family protein

ATCG00900 Ribosomal protein S7p/S5e family protein

AT4G28706 pfkB-like carbohydrate kinase family protein

AT5G11770 NADH-ubiquinone oxidoreductase $20 \mathrm{kDa}$ subunit

AT5G14670 ADP-ribosylation factor A1B

AT1G41880 Ribosomal protein L35Ae family protein

AT5G57290 60S acidic ribosomal protein family

AT2G19730 Ribosomal L28e protein family

AT1G74970 ribosomal protein S9

AT4G23670 Polyketide cyclase/dehydrase and lipid transport superfamily prot.

AT2G04390 Ribosomal S17 family protein

AT2G20260 photosystem I subunit E-2

AT4G27090 Ribosomal protein L14

AT3G04920 Ribosomal protein S24e family protein

AT2G39460 ribosomal protein L23AA

AT2G34480 Ribosomal protein L18ae/LX family protein

AT4G34870 rotamase cyclophilin 5

ATCG00780 ribosomal protein L14

\begin{tabular}{|c|c|c|c|c|}
\hline 19 & 3 & 100 & 6.64 & 7.4E-17 \\
\hline 39 & 9 & 100 & 6.64 & 7.4E-17 \\
\hline 27 & 4 & 100 & 6.64 & 7.4E-17 \\
\hline 46 & 5 & 100 & 6.64 & 7.4E-17 \\
\hline 37 & 2 & 100 & 6.64 & 7.4E-17 \\
\hline 28 & 2 & 100 & 6.64 & 7.4E-17 \\
\hline 6 & 2 & 100 & 6.64 & 7.4E-17 \\
\hline 20 & 3 & 100 & 6.64 & 7.4E-17 \\
\hline 13 & 2 & 100 & 6.64 & 7.4E-17 \\
\hline 32 & 2 & 100 & 6.64 & 7.4E-17 \\
\hline 13 & 2 & 100 & 6.64 & 7.4E-17 \\
\hline 15 & 2 & 100 & 6.64 & 7.4E-17 \\
\hline 21 & 3 & 100 & 6.64 & 7.4E-17 \\
\hline 31 & 2 & 100 & 6.64 & 7.4E-17 \\
\hline 52 & 3 & 100 & 6.64 & 7.4E-17 \\
\hline 21 & 2 & 100 & 6.64 & 7.4E-17 \\
\hline 21 & 3 & 100 & 6.64 & 7.4E-17 \\
\hline 9 & 2 & 100 & 6.64 & 7.4E-17 \\
\hline 17 & 2 & 100 & 6.64 & 7.4E-17 \\
\hline 15 & 2 & 100 & 6.64 & $7.4 \mathrm{E}-17$ \\
\hline 11 & 2 & 100 & 6.64 & 7.4E-17 \\
\hline 28 & 4 & 100 & 6.64 & 7.4E-17 \\
\hline 12 & 2 & 100 & 6.64 & 7.4E-17 \\
\hline 45 & 2 & 100 & 6.64 & 7.4E-17 \\
\hline 42 & 3 & 100 & 6.64 & 7.4E-17 \\
\hline 27 & 2 & 100 & 6.64 & 7.4E-17 \\
\hline 12 & 2 & 100 & 6.64 & 7.4E-17 \\
\hline 23 & 2 & 100 & 6.64 & 7.4E-17 \\
\hline 34 & 2 & 100 & 6.64 & 7.4E-17 \\
\hline 32 & 3 & 100 & 6.64 & 7.4E-17 \\
\hline 50 & 8 & 94.88 & 6.57 & 7.4E-17 \\
\hline 58 & 8 & 81.46 & 6.35 & 7.4E-17 \\
\hline 47 & 10 & 41.58 & 5.38 & 7.4E-17 \\
\hline 22 & 3 & 34.62 & 5.11 & $7.4 \mathrm{E}-17$ \\
\hline 8 & 2 & 32.79 & 5.04 & $7.4 \mathrm{E}-17$ \\
\hline 36 & 2 & 32.30 & 5.01 & 7.4E-17 \\
\hline 31 & 4 & 30.62 & 4.94 & 7.4E-17 \\
\hline 48 & 7 & 28.06 & 4.81 & 7.4E-17 \\
\hline 64 & 2 & 27.79 & 4.8 & 7.4E-17 \\
\hline 23 & 4 & 27.45 & 4.78 & $7.4 \mathrm{E}-17$ \\
\hline 22 & 4 & 24.11 & 4.59 & 7.4E-17 \\
\hline 48 & 5 & 23.35 & 4.55 & 7.4E-17 \\
\hline 43 & 4 & 21.97 & 4.46 & 7.4E-17 \\
\hline 68 & 3 & 21.51 & 4.43 & 7.4E-17 \\
\hline 26 & 4 & 20.97 & 4.39 & 7.4E-17 \\
\hline 31 & 5 & 19.13 & 4.26 & 7.4E-17 \\
\hline 69 & 7 & 17.71 & 4.15 & $7.4 \mathrm{E}-17$ \\
\hline 29 & 2 & 15.77 & 3.98 & $7.4 \mathrm{E}-17$ \\
\hline 46 & 9 & 15.76 & 3.98 & 7.4E-17 \\
\hline 8 & 2 & 15.60 & 3.96 & 7.4E-17 \\
\hline 31 & 2 & 13.09 & 3.71 & $6.5 \mathrm{E}-15$ \\
\hline 43 & 4 & 12.73 & 3.67 & 1.3E-14 \\
\hline 8 & 2 & 12.48 & 3.64 & 2.3E-14 \\
\hline 45 & 6 & 12.15 & 3.6 & 4.3E-14 \\
\hline 50 & 2 & 12.14 & 3.6 & $4.5 \mathrm{E}-14$ \\
\hline 51 & 5 & 11.74 & 3.55 & 1.0E-13 \\
\hline 69 & 7 & 11.58 & 3.53 & $1.4 \mathrm{E}-13$ \\
\hline 28 & 5 & 11.36 & 3.51 & $2.2 \mathrm{E}-13$ \\
\hline 37 & 4 & 11.25 & 3.49 & $2.7 \mathrm{E}-13$ \\
\hline 64 & 15 & 11.18 & 3.48 & $3.2 \mathrm{E}-13$ \\
\hline 33 & 3 & 10.78 & 3.43 & $7.5 \mathrm{E}-13$ \\
\hline 33 & 4 & 10.42 & 3.38 & 1.6E-12 \\
\hline 16 & 3 & 10.39 & 3.38 & $1.8 \mathrm{E}-12$ \\
\hline 46 & 8 & 10.14 & 3.34 & $3.1 \mathrm{E}-12$ \\
\hline 17 & 6 & 10.01 & 3.32 & 4.1E-12 \\
\hline 9 & 2 & 8.99 & 3.17 & $4.5 \mathrm{E}-11$ \\
\hline 63 & 13 & 8.79 & 3.14 & 7.3E-11 \\
\hline 16 & 2 & 8.30 & 3.05 & $2.5 \mathrm{E}-10$ \\
\hline 22 & 2 & 8.24 & 3.04 & $2.8 \mathrm{E}-10$ \\
\hline 25 & 3 & 8.24 & 3.04 & $2.8 \mathrm{E}-10$ \\
\hline 31 & 6 & 8.19 & 3.03 & $3.2 \mathrm{E}-10$ \\
\hline 64 & 9 & 7.95 & 2.99 & $5.8 \mathrm{E}-10$ \\
\hline 30 & 5 & 7.80 & 2.96 & $8.5 \mathrm{E}-10$ \\
\hline 46 & 2 & 7.80 & 2.96 & 8.7E-10 \\
\hline 56 & 6 & 7.59 & 2.92 & 1.5E-09 \\
\hline 31 & 3 & 6.93 & 2.79 & 8.8E-09 \\
\hline 42 & 11 & 6.93 & 2.79 & 8.8E-09 \\
\hline 36 & 5 & 6.84 & 2.77 & 1.1E-08 \\
\hline 42 & 5 & 6.73 & 2.75 & 1.5E-08 \\
\hline 52 & 5 & 6.66 & 2.74 & $1.9 \mathrm{E}-08$ \\
\hline
\end{tabular}




\begin{tabular}{|c|c|c|c|c|c|c|}
\hline AT1G70600 & Ribosomal protein L18e/L15 superfamily protein & 43 & 6 & 6.56 & 2.71 & $2.5 \mathrm{E}-08$ \\
\hline AT5G63310 & nucleoside diphosphate kinase 2 & 43 & 9 & 5.61 & 2.49 & 4.1E-07 \\
\hline AT3G48930 & Nucleic acid-binding, OB-fold-like protein & 49 & 6 & 5.54 & 2.47 & $5.2 \mathrm{E}-07$ \\
\hline AT5G23820 & MD-2-related lipid recognition domain-containing protein & 20 & 5 & 5.43 & 2.44 & 7.3E-07 \\
\hline AT3G25920 & ribosomal protein L15 & 36 & 10 & 5.20 & 2.38 & $1.5 \mathrm{E}-06$ \\
\hline AT5G52650 & RNA binding Plectin/S10 domain-containing protein & 25 & 5 & 5.20 & 2.38 & $1.5 \mathrm{E}-06$ \\
\hline AT1G22780 & Ribosomal protein S13/S18 family & 39 & 6 & 4.99 & 2.32 & $2.9 \mathrm{E}-06$ \\
\hline AT5G59870 & histone $\mathrm{H} 2 \mathrm{~A} 6$ & 52 & 5 & 4.96 & 2.31 & $3.2 \mathrm{E}-06$ \\
\hline AT1G73990 & signal peptide peptidase & 3 & 2 & 4.83 & 2.27 & 1.3E-04 \\
\hline AT4G34260 & 1,2-alpha-L-fucosidases & 3 & 2 & 4.70 & 2.23 & 7.4E-06 \\
\hline AT5G13120 & cyclophilin $20-2$ & 22 & 4 & 4.70 & 2.23 & 7.6E-06 \\
\hline AT5G13410 & FKBP-like peptidyl-prolyl cis-trans isomerase family protein & 29 & 5 & 4.67 & 2.22 & $1.2 \mathrm{E}-05$ \\
\hline AT5G41520 & RNA binding Plectin/S10 domain-containing protein & 41 & 8 & 4.25 & 2.09 & 3.4E-05 \\
\hline AT3G56340 & Ribosomal protein S26e family protein & 50 & 5 & 4.21 & 2.07 & $3.9 \mathrm{E}-05$ \\
\hline AT2G30140 & UDP-Glycosyltransferase superfamily protein & 6 & 2 & 4.15 & 2.05 & $9.1 \mathrm{E}-05$ \\
\hline AT1G27400 & Ribosomal protein L22p/L17e family protein & 26 & 5 & 3.91 & 1.97 & 1.1E-04 \\
\hline AT5G27850 & Ribosomal protein L18e/L15 superfamily protein & 59 & 5 & 3.82 & 1.93 & $1.5 \mathrm{E}-04$ \\
\hline AT3G62030 & rotamase CYP 4 & 39 & 9 & 3.47 & 1.79 & 5.4E-04 \\
\hline AT3G52960 & Thioredoxin superfamily protein-PRXIIE & 53 & 12 & 3.42 & 1.77 & $6.6 \mathrm{E}-04$ \\
\hline AT5G08410 & ferredoxin/thioredoxin reductase subunit A (variable subunit) 2 & 8 & 2 & 3.39 & 1.76 & 1.7E-03 \\
\hline AT5G52840 & $\mathrm{NADH}$-ubiquinone oxidoreductase-related & 24 & 3 & 3.26 & 1.71 & 1.1E-03 \\
\hline AT1G77940 & Ribosomal protein L7Ae/L30e/S12e/Gadd45 family protein & 36 & 2 & 3.21 & 1.68 & 1.4E-03 \\
\hline AT2G35370 & glycine decarboxylase complex $\mathrm{H}$ & 30 & 2 & 3.19 & 1.67 & $1.5 \mathrm{E}-03$ \\
\hline AT1G73230 & Nascent polypeptide-associated complex NAC & 41 & 3 & 3.17 & 1.67 & $5.5 \mathrm{E}-03$ \\
\hline AT5G23900 & Ribosomal protein $\mathrm{L} 13 \mathrm{e}$ family protein & 33 & 2 & 0.30 & -1.72 & 8.4E-04 \\
\hline AT5G64100 & Peroxidase superfamily protein & 44 & 14 & 0.29 & -1.78 & 5.2E-04 \\
\hline AT1G47600 & beta glucosidase 34 & 23 & 2 & 0.01 & -6.64 & $7.4 \mathrm{E}-17$ \\
\hline AT4G30810 & serine carboxypeptidase-like 29 & 4 & 2 & 0.01 & -6.64 & $7.4 \mathrm{E}-17$ \\
\hline AT3G57520 & seed imbibition 2 & 6 & 4 & 0.01 & -6.64 & $7.4 \mathrm{E}-17$ \\
\hline AT4G08950 & Phosphate-responsive 1 family protein & 9 & 2 & 0.01 & -6.64 & $7.4 \mathrm{E}-17$ \\
\hline AT3G54360 & zinc ion binding & 6 & 2 & 0.01 & -6.64 & $7.4 \mathrm{E}-17$ \\
\hline AT1G33610 & Leucine-rich repeat (LRR) family protein & 5 & 2 & 0.01 & -6.64 & $7.4 \mathrm{E}-17$ \\
\hline AT2G28190 & copper/zinc superoxide dismutase 2 & 24 & 3 & 0.01 & -6.64 & $7.4 \mathrm{E}-17$ \\
\hline AT2G30170 & Protein phosphatase $2 \mathrm{C}$ family protein & 21 & 5 & 0.01 & -6.64 & $7.4 \mathrm{E}-17$ \\
\hline AT5G60360 & aleurain-like protease & 13 & 3 & 0.01 & -6.64 & $7.4 \mathrm{E}-17$ \\
\hline AT2G18980 & Peroxidase superfamily protein & 24 & 3 & 0.01 & -6.64 & 7.4E-17 \\
\hline AT5G63190 & MA3 domain-containing protein & 6 & 2 & 0.01 & -6.64 & $7.4 \mathrm{E}-17$ \\
\hline
\end{tabular}

Suppl. Table 4.11. Proteins detected as differentially abundant in the three single amiR mutants under normal conditions.

\begin{tabular}{clcccccc} 
& & \multicolumn{2}{c}{ amiR23.5 } & \multicolumn{2}{c}{ amiR23.6 } & \multicolumn{2}{c}{ amiR26.5 } \\
\cline { 3 - 8 } Locus & Protein name & $\begin{array}{c}\text { Fold } \\
\text { change } \\
\left(\mathbf{l o g}_{2}\right)\end{array}$ & $\begin{array}{c}\text { Adj. P- } \\
\text { Value }\end{array}$ & $\begin{array}{c}\text { Fold } \\
\text { change } \\
\left(\mathbf{l o g}_{2}\right)\end{array}$ & $\begin{array}{c}\text { Adj. P- } \\
\text { Value }\end{array}$ & $\begin{array}{c}\text { Fold } \\
\text { change } \\
\left(\mathbf{l o g}_{2}\right)\end{array}$ & $\begin{array}{c}\text { Adj. P- } \\
\text { Value }\end{array}$ \\
\hline AT5G47190 & $\begin{array}{l}\text { Ribosomal protein } \\
\text { L19 family protein }\end{array}$ & 6.64 & $1.1 \mathrm{E}-16$ & 6.64 & $6.4 \mathrm{E}-17$ & 6.64 & $6.3 \mathrm{E}-17$ \\
\hline AT1G74970 & ribosomal protein S9 & 6.64 & $1.1 \mathrm{E}-16$ & 6.64 & $6.4 \mathrm{E}-17$ & 6.64 & $6.3 \mathrm{E}-17$ \\
\hline AT3G45140 & lipoxygenase 2 & 1.14 & $3.0 \mathrm{E}-08$ & 1.95 & $2.8 \mathrm{E}-13$ & 1.65 & $1.8 \mathrm{E}-08$ \\
\hline AT5G23900 & $\begin{array}{l}\text { Ribosomal protein } \\
\text { L13e family protein }\end{array}$ & -1.67 & $2.4 \mathrm{E}-06$ & -2.45 & $4.8 \mathrm{E}-06$ & -6.64 & $6.3 \mathrm{E}-17$ \\
\hline AT1G62480 & $\begin{array}{l}\text { Vacuolar calcium- } \\
\text { binding protein- } \\
\text { related }\end{array}$ & -6.64 & $1.1 \mathrm{E}-16$ & -6.64 & $6.4 \mathrm{E}-17$ & -6.64 & $6.3 \mathrm{E}-17$ \\
\hline
\end{tabular}


Suppl. Table 4.12. Proteins detected as differentially abundant in the three single amiR mutants after heat shock treatment.

\begin{tabular}{llcccccc} 
& & \multicolumn{2}{c}{ amiR23.5 } & \multicolumn{2}{c}{ amiR23.6 } & \multicolumn{2}{c}{ amiR26.5 } \\
\cline { 3 - 7 } Locus & \multicolumn{1}{c}{ Protein name } & $\begin{array}{c}\text { Fold } \\
\text { change } \\
\left(\mathbf{l o g}_{2}\right)\end{array}$ & $\begin{array}{c}\text { Adj. P- } \\
\text { Value }\end{array}$ & $\begin{array}{c}\text { Fold } \\
\text { change } \\
\left(\mathbf{l o g}_{2}\right)\end{array}$ & $\begin{array}{c}\text { Adj. P- } \\
\text { Value }\end{array}$ & $\begin{array}{c}\text { Fold } \\
\text { change } \\
\left(\mathbf{l o g}_{2}\right)\end{array}$ & $\begin{array}{c}\text { Adj. P- } \\
\text { Value }\end{array}$ \\
\hline AT4G40030 & $\begin{array}{l}\text { Histone superfamily } \\
\text { protein }\end{array}$ & 2.71 & $1.2 \mathrm{E}-09$ & 4.52 & $8.2 \mathrm{E}-17$ & 4.62 & $7.0 \mathrm{E}-17$ \\
\hline AT4G34870 & $\begin{array}{l}\text { rotamase cyclophilin } \\
5\end{array}$ & 2.49 & $4.1 \mathrm{E}-12$ & 3.09 & $3.2 \mathrm{E}-13$ & 2.17 & $7.4 \mathrm{E}-14$ \\
\hline AT4G09320 & $\begin{array}{l}\text { Nucleoside } \\
\text { diphosphate kinase } \\
\text { family protein }\end{array}$ & 2.07 & $7.8 \mathrm{E}-05$ & 6.2 & $8.2 \mathrm{E}-17$ & 6.27 \\
\hline AT5G41520 & $\begin{array}{l}\text { RNA binding } \\
\text { Plectin/S10 domain- } \\
\text { containing protein }\end{array}$ & 1.68 & $4.5 \mathrm{E}-04$ & 1.99 & $4.9 \mathrm{E}-06$ & 2.02 \\
\hline AT1G69620 & $\begin{array}{l}\text { ribosomal protein } \\
\text { L34 }\end{array}$ & 1.47 & $2.6 \mathrm{E}-03$ & 3.35 & $1.8 \mathrm{E}-15$ & 3.82 \\
\hline
\end{tabular}


